\title{
One-Pot Synthesis and Conformational Analysis of 6-Membered Cyclic lodonium Salts
}

Lucien Caspers, Julian Spils, Mattis Damrath, Enno Lork, Boris Nachtsheim

Submitted date: 02/05/2020 - Posted date: 05/05/2020

Licence: CC BY-NC-ND 4.0

Citation information: Caspers, Lucien; Spils, Julian; Damrath, Mattis; Lork, Enno; Nachtsheim, Boris (2020): One-Pot Synthesis and Conformational Analysis of 6-Membered Cyclic lodonium Salts. ChemRxiv. Preprint. https://doi.org/10.26434/chemrxiv.12234665.v1

In this article we describe an efficient approach for the synthesis of cyclic diaryliodonium salts. The method is based on benzyl alcohols as starting materials and consists of an Friedel-Crafts-arylation/oxidation sequence. Besides a deep optimization, particluar focusing on the choice and ratios of the utilized Bronsted-acids and oxidants, we explore the substrate scope of this transformation. We also discuss an interesting isomerism of cyclic iodonium salts substituted with aliphatic substituents at the bridge head carbon.

File list (3)

ChemRXIV_SI.pdf (2.43 MiB)

view on ChemRxiv • download file

graphical abstract.jpg (50.04 KiB)

view on ChemRxiv • download file

manuscript_Chemrxiv.pdf (590.20 KiB)

view on ChemRxiv - download file 


\section{Supporting Information}

\section{One-Pot Synthesis and Conformational Analysis of 6-Membered Cyclic lodonium Salts}

Lucien D. Caspers ${ }^{a}$, Julian Spils ${ }^{a}$, Mattis Damrath ${ }^{a}$, Enno Lork ${ }^{b}$ and\# Boris J. Nachtsheim ${ }^{a *}$

aAddress: Institute for Organic and Analytical Chemistry, University of Bremen, 28359 Bremen, Germany, phone: +49 421 218-63113

${ }^{b}$ Address: Institute for Inorganic Chemistry and Crystallography, University of Bremen, 28359 Bremen, Germany, phone: +49 421 218-63155

Prof. Dr. Boris J. Nachtsheim, nachtsheim@uni-bremen.de 


\section{Table of Contents}

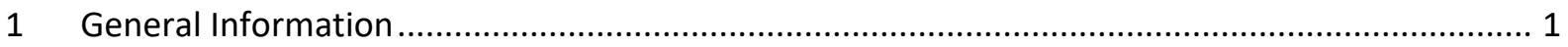

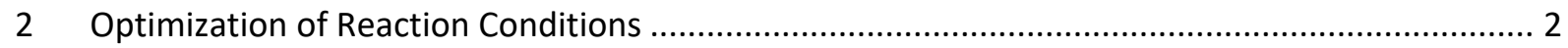

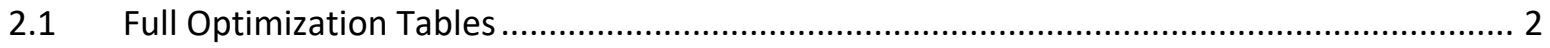

2.1.1 Preliminary Method with $m$ CPBA as Oxidant ............................................................ 2

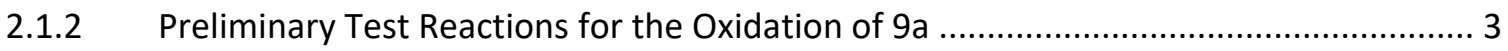

2.1.3 Final Method for Primary Alcohols with Oxone ${ }^{\circledR}$ as Oxidant ......................................... 4

2.1.4 Preliminary Method for Secondary Alcohols with $m C P B A$ as Oxidant.......................... 6

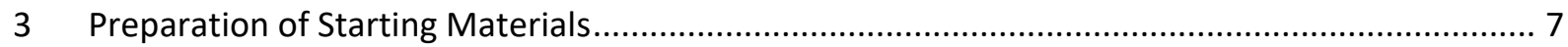

3.1 General Procedures for the Synthesis of the Starting Materials........................................ 7

3.1.1 Sandmeyer-type lodination of Anthranilic acids (GP1a) .............................................. 7

3.1.2 Sandmeyer-type lodination of Anthranilic acids (GP1b) ............................................ 7

3.1.3 Borane-mediated Reduction of Carboxylic acids (GP2) ............................................ 8

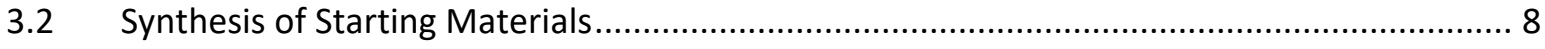

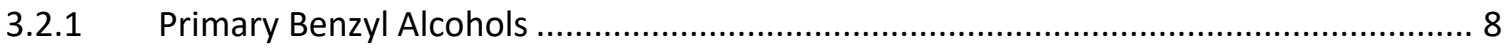

3.2.2 Derivatives of Primary Benzyl Alcohols ........................................................................ 14

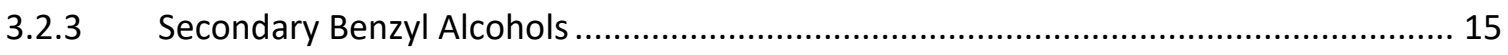

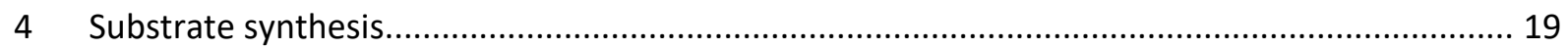

4.1 General Procedures for the One-Pot Reaction Utilizing Primary Benzyl Alcohols and

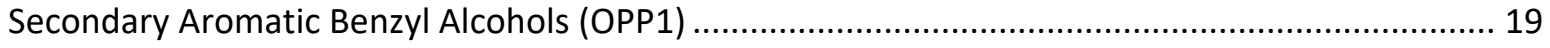

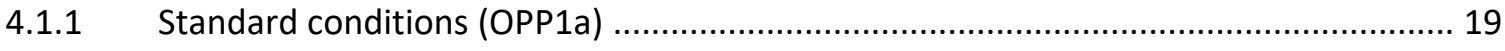

4.1.2 Conditions for benzyl alcohols with electron withdrawing groups (OPP1b)................ 19

4.1.3 Conditions for benzyl alcohols with electron withdrawing groups (OPP1c) ................. 20

4.2 Substrates from Primary Benzyl Alcohols and Secondary Aromatic Benzyl Alcohols ........... 20

4.3 General Procedures for the One-Pot Reaction Utilizing Secondary Aliphatic Benzyl Alcohols

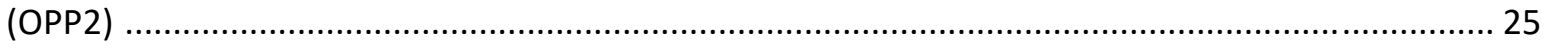

4.4 Substrates Derived from Secondary Aliphatic Benzyl Alcohols.......................................... 25

4.5 Substrates that could not be successfully synthesized .................................................. 29

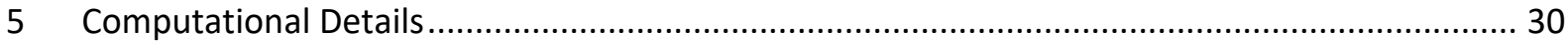

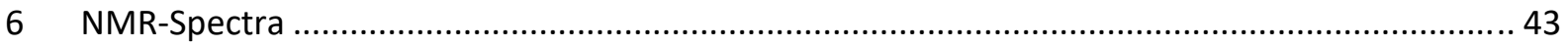

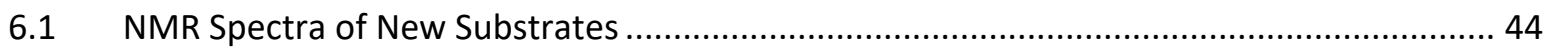

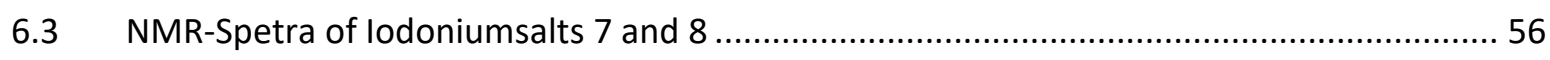

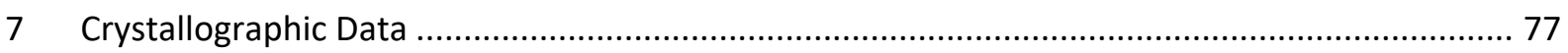

8 Literature 
List of Figures

Figure S1: Substrates that could not successfully be synthesized with OPP1 or OPP2 ..................... 29

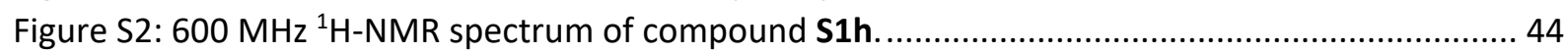

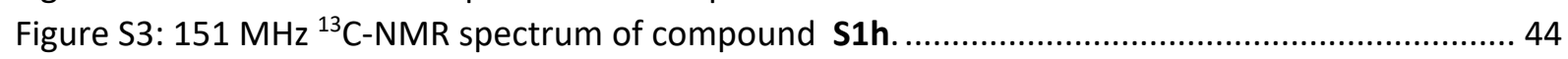

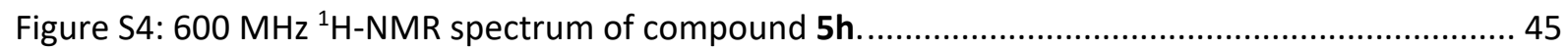

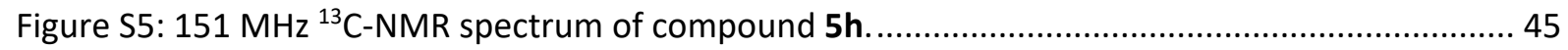

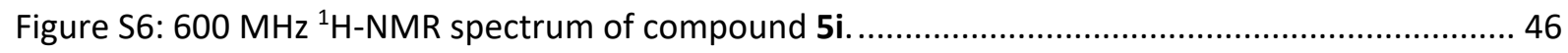

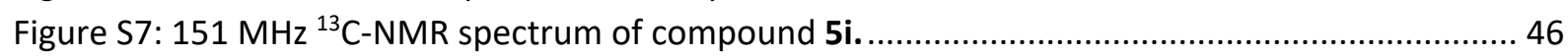

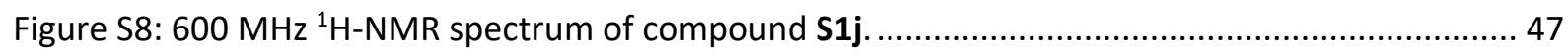

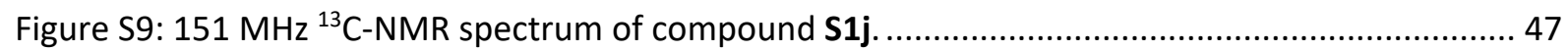

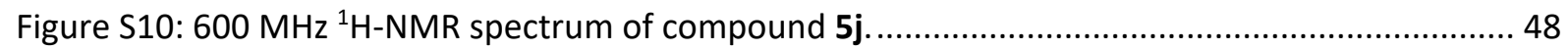

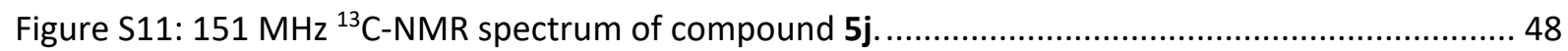

Figure S12: $600 \mathrm{MHz}{ }^{1} \mathrm{H}-\mathrm{NMR}$ spectrum of methyl 5-cyano-2-iodobenzoate. .................................... 49

Figure S13: $151 \mathrm{MHz}{ }^{13} \mathrm{C}-\mathrm{NMR}$ spectrum of methyl 5-cyano-2-iodobenzoate. ................................... 49

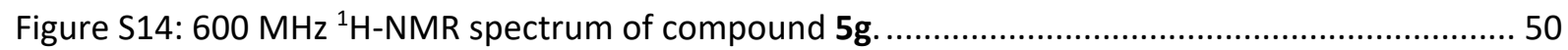

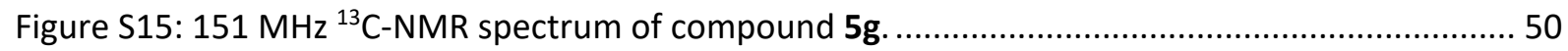

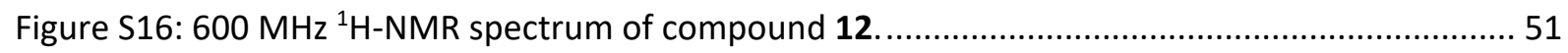

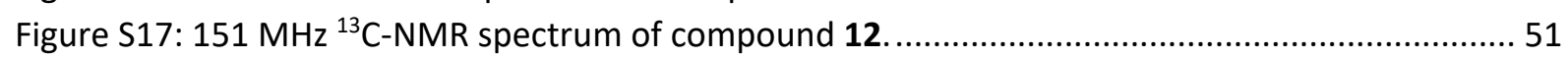

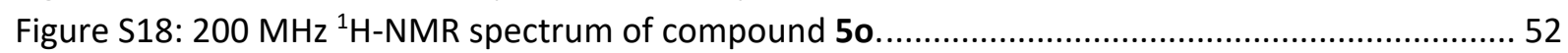

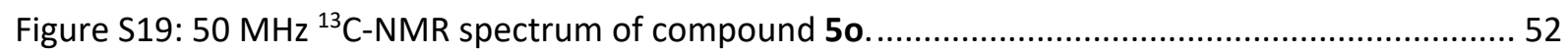

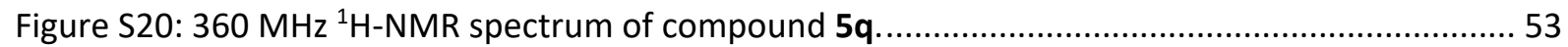

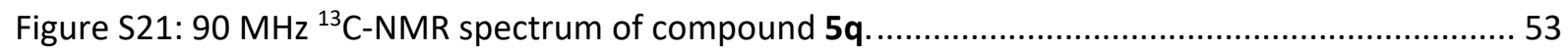

Figure S22: $360 \mathrm{MHz}{ }^{1} \mathrm{H}-\mathrm{NMR}$ spectrum of compound (5-chloro-2-iodophenyl)(2-

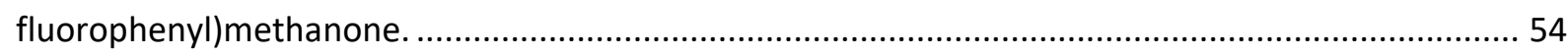

Figure S23: $90 \mathrm{MHz} \quad{ }^{13} \mathrm{C}-\mathrm{NMR}$ spectrum of compound (5-chloro-2-iodophenyl)(2-

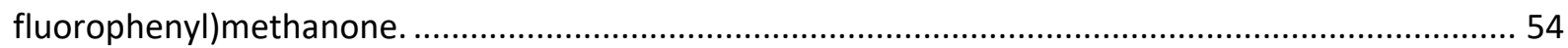

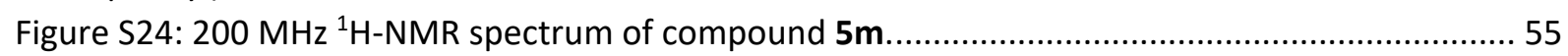

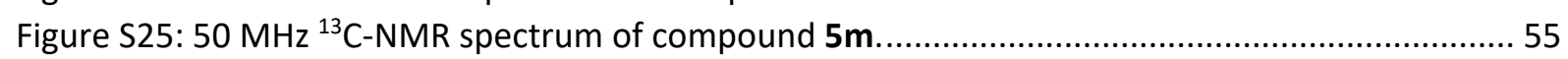

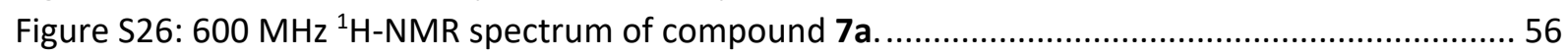

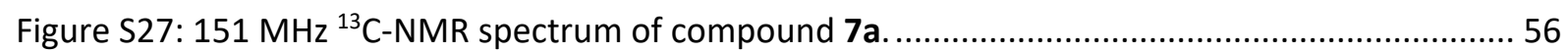

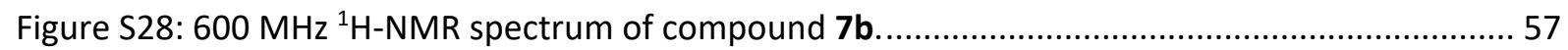

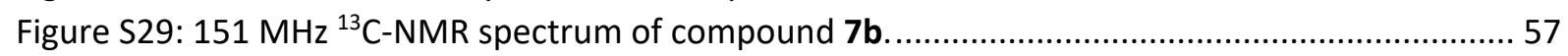

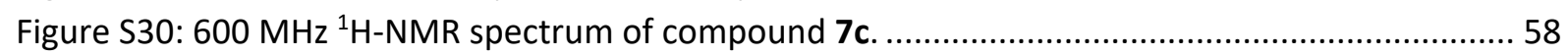

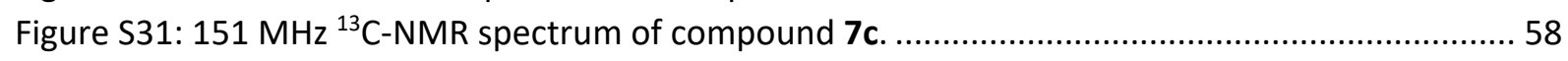

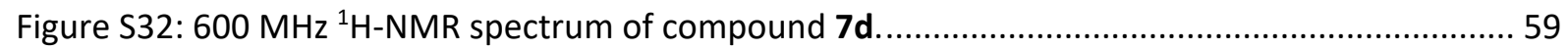

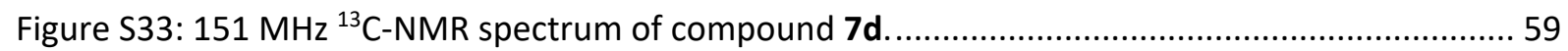

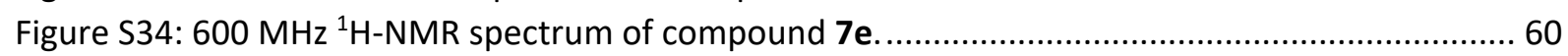

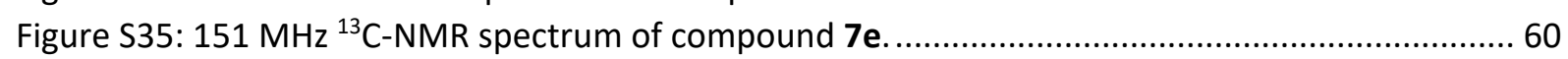

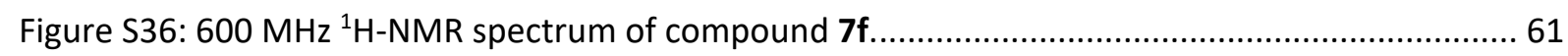

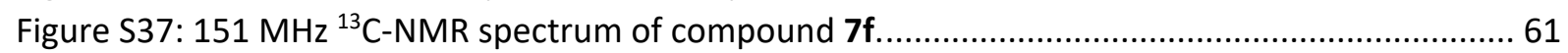

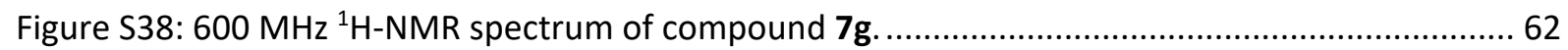

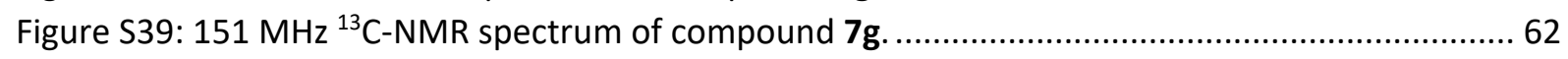

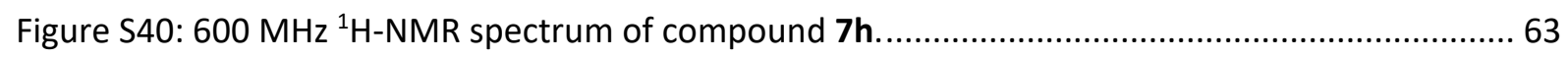

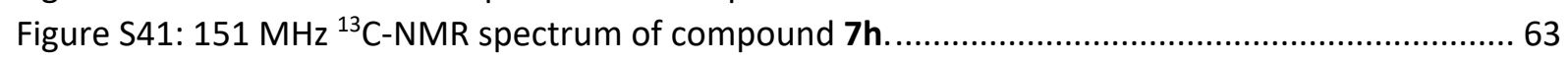

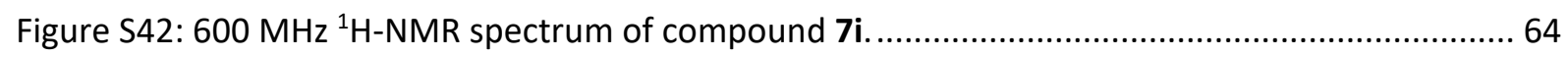

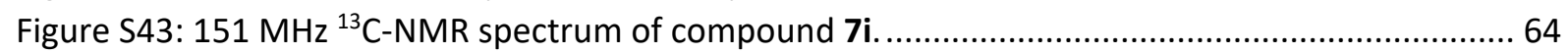

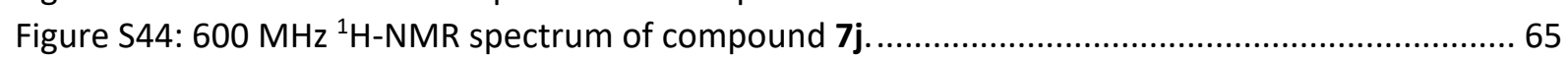

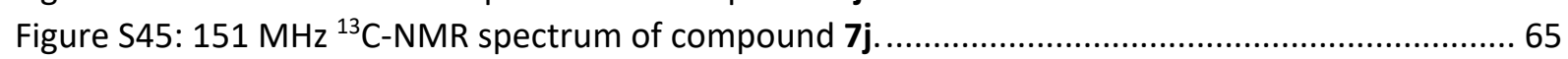




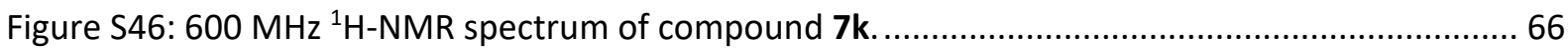

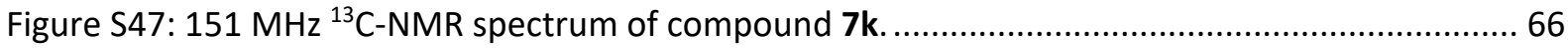

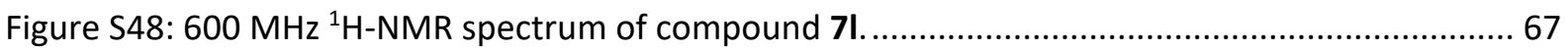

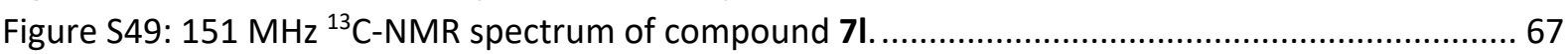

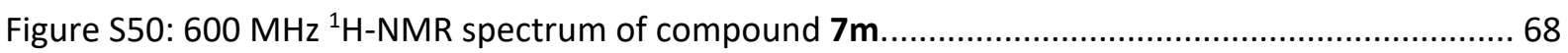

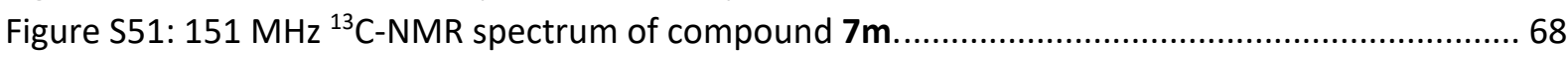

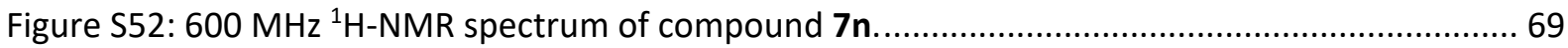

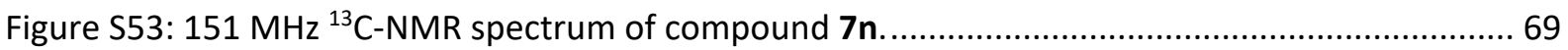

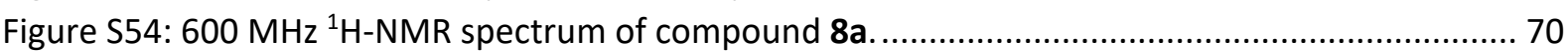

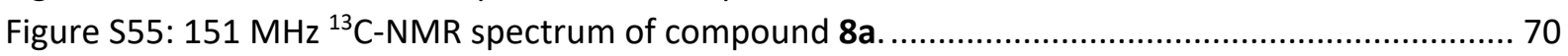

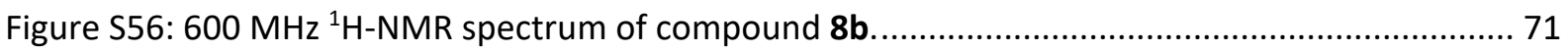

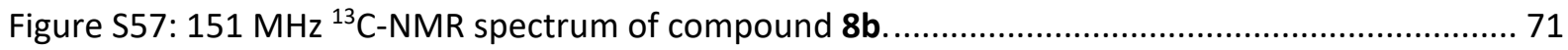

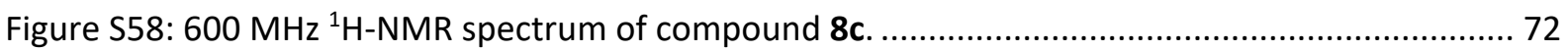

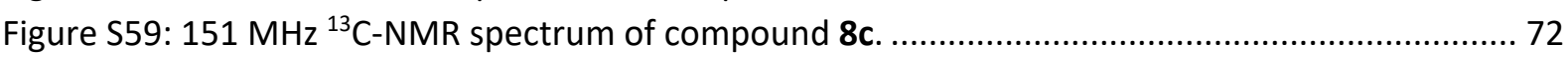

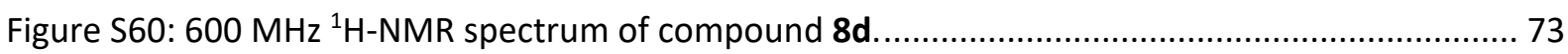

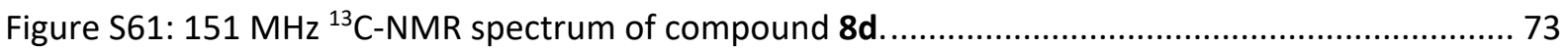

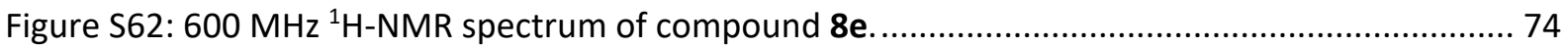

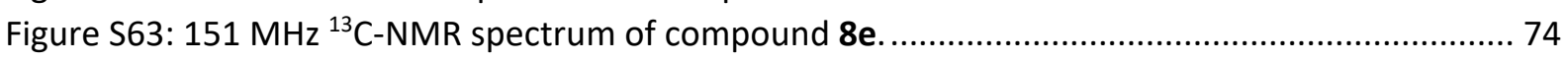

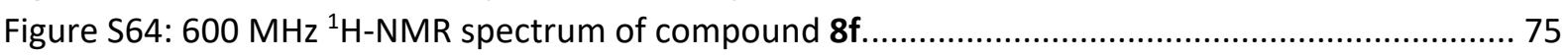

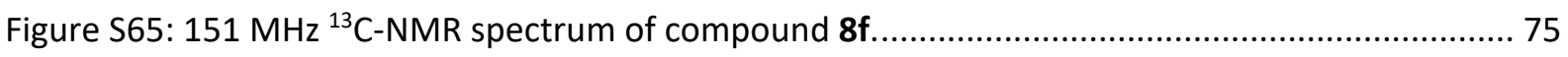

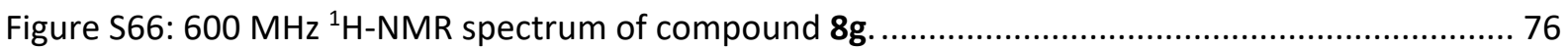

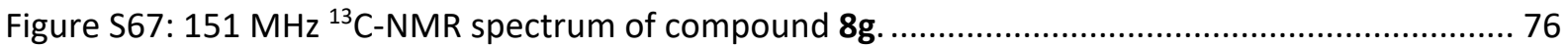

\section{List of Tables}

Table S1: Preliminary aotimization of the one-pot synthesis of $10 \mathrm{H}$-dibenzo[b,e]iodinin-5-ium

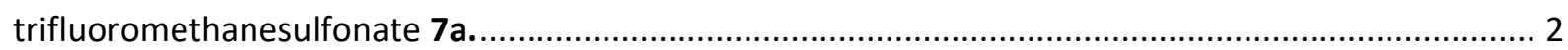

Table S2: Optimization for the oxidation reaction of $9 \mathrm{a}$ for the synthesis of $10 \mathrm{H}$-dibenzo[b,e]iodinin-

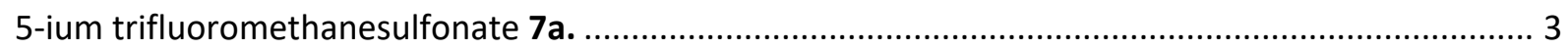
Table S3: Final optimization of the one-pot synthesis of $10 \mathrm{H}$-dibenzo[b,e]iodinin-5-ium

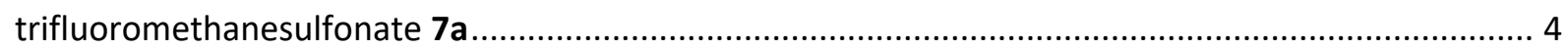

Table S4: Reoptimization for benzylic alcohols with electron-withdrawing groups.............................. 5

Table S5: Reoptimization for secondary benzylic alcohols with toluene ........................................... 6

Table S6: Energies for iodonium salt conformers as calculated on CPCM(DCM)/RI-DSD-BLYP/TZVPP//RI-

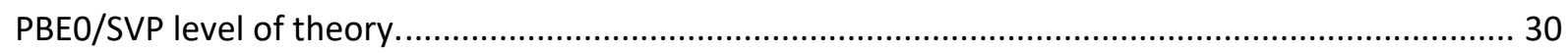

Table S7: Energies for iodonium salt conformers as calculated on CPCM(DMSO)/RI-DSD-

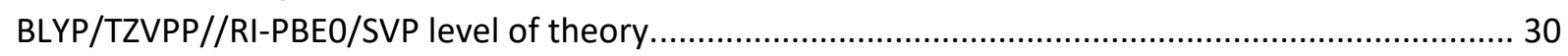

Table S8: Energies for iodonium salt conformers as calculated on CPCM(MeOH)/RI-DSD-

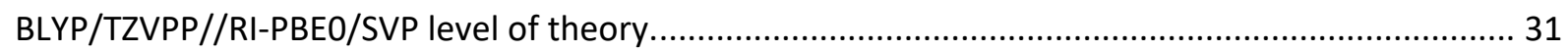

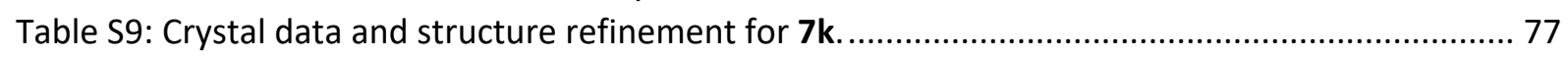

Table S10: Fractional Atomic Coordinates $\left(\times 10^{4}\right)$ and Equivalent Isotropic Displacement Parameters $\left(\AA^{2} \times 10^{3}\right)$ for $7 \mathbf{k} . U_{\text {eq }}$ is defined as $1 / 3$ of of the trace of the orthogonalised $U_{\text {IJ }}$ tensor. ....................... 78 Table S11: Anisotropic Displacement Parameters $\left(\AA^{2} \times 10^{3}\right)$ for $\mathbf{7 k}$. The Anisotropic displacement factor

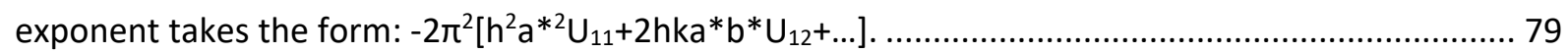

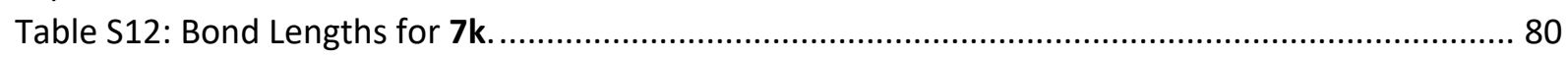

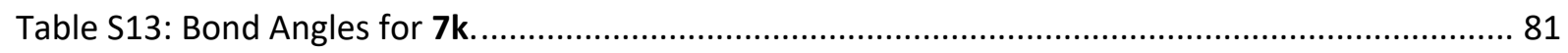

Table S14: Hydrogen Atom Coordinates $\left(\AA \times 10^{4}\right)$ and Isotropic Displacement Parameters $\left(\AA^{2} \times 10^{3}\right)$ for 7k. 


\section{General Information}

Unless otherwise noted, all reactions were carried out under air. Reactions with chemicals sensitive to moisture or oxygen were carried out under a nitrogen atmosphere using standard Schlenk techniques. All chemicals were purchased from commercial suppliers and either used as received or purified according to "Purification of Laboratory Chemicals". ${ }^{[1]}$ Anhydrous tetrahydrofuran (THF) was obtained from an Inert PS-MD-6 solvent purification system. All other solvents were dried using standard methods if necessary. ${ }^{[1]}$

Yields refer to isolated yields of compounds estimated to be $>95 \%$ pure as determined by ${ }^{1} \mathrm{H}-\mathrm{NMR}$ spectroscopy.

Thin layer chromatography was performed on fluorescence indicator marked precoated silica gel 60 plates (Macherey-Nagel, ALUGRAM Xtra SIL G/UV254) and visualized by UV light ( $254 \mathrm{~nm} / 366 \mathrm{~nm}$ ). Flash column chromatography was performed on silica gel $(0.040-0.063 \mathrm{~mm})$ with the solvents given in the procedures.

NMR spectra were recorded on a Bruker Avance DPX-200, a Bruker Avance 360WB spectrometer, a Bruker Avance Neo $600 \mathrm{MHz}$ spectrometer with BBO probe head and a Bruker Avance Neo $600 \mathrm{MHz}$ spectrometer with TXI probe head at $23^{\circ} \mathrm{C}$. Chemical shifts for ${ }^{1} \mathrm{H}-\mathrm{NMR}$ spectra are reported as $\delta$ (parts per million) relative to the residual proton signal of $\mathrm{CDCl}_{3}$ at $7.26 \mathrm{ppm}(\mathrm{s}), \mathrm{DMSO}-d_{6}$ at $2.50 \mathrm{ppm}$ (quint) or $\mathrm{MeOH}-d_{4}$ at $3.31 \mathrm{ppm}$ (quint). Chemical shifts for ${ }^{13} \mathrm{C}-\mathrm{NMR}$ spectra are reported as $\delta$ (parts per million) relative to the signal of $\mathrm{CDCl}_{3}$ at $77.0 \mathrm{ppm}\left(\mathrm{t}\right.$ ), DMSO- $d_{6}$ at $39.5 \mathrm{ppm}$ (sept) or $\mathrm{MeOH}-d_{4}$ at $49.0 \mathrm{ppm}$ (sept). Chemical shifts for ${ }^{19} \mathrm{~F}-\mathrm{NMR}$ spectra are reported as $\delta$ (parts per million) relative to the signal of $\mathrm{Si}\left(\mathrm{CH}_{3}\right)_{4}$ at $0.0 \mathrm{ppm}$. The following abbreviations are used to describe splitting patterns: br. = broad, $\mathrm{s}=$ singlet, $\mathrm{d}=$ doublet, $\mathrm{t}=$ triplet, $\mathrm{q}=$ quartet, quint $=$ quintet, sept $=$ septet, $\mathrm{m}=$ multiplet. Coupling constants $J$ are given in $\mathrm{Hz}$.

ESI and APCI mass spectra were recorded on an Advion Expression CMS ${ }^{\llcorner}$via ASAP probe or direct inlet. Low resolution El mass spectra were recorded on an Agilent 5977A Series GC/MSD system. High resolution (HR) El mass spectra were recorded on a double focusing mass spectrometer ThermoQuest MAT $95 \mathrm{XL}$ from Finnigan MAT. HR-ESI and HR-APCI mass spectra were recorded on a Bruker Impact II. All Signals are reported with the quotient from mass to charge $\mathrm{m} / \mathrm{z}$.

IR spectra were recorded on a Nicolet Thermo iS10 scientific spectrometer with a diamond ATR unit. The absorption bands $\tilde{v}$ are reported in $\mathrm{cm}^{-1}$.

Melting points of solids were measured on a Büchi M-5600 Melting Point apparatus and are uncorrected. The measurements were performed with a heating rate of $2^{\circ} \mathrm{C} / \mathrm{min}$ and the melting point temperatures $T$ are reported in ${ }^{\circ} \mathrm{C}$.

Intensity data of suitable single crystals were collected on a Bruker Venture D8 diffractometer at 100 $\mathrm{K}$ with Mo- $\mathrm{K}_{\alpha}(0.71073 \AA)$ radiation. All structures were solved by direct methods and refined based on F2 by use of the SHELX program package as implemented in Olex 2.2 All non-hydrogen atoms were refined using anisotropic displacement parameters. Hydrogen atoms attached to carbon atoms were included in geometrically calculated positions using a rigid model. Figures were created using Olex2. Crystallographic data for the structural analyses have been deposited with the Cambridge Crystallographic Data Centre. Copies of this information may be obtained free of charge from The Director, CCDC, 12 Union Road, Cambridge CB2 1EZ, UK (Fax: +44-1223-336033; e-mail: deposit@ccdc.cam.ac.uk or http://www.ccdc.cam.ac.uk). 


\section{Optimization of Reaction Conditions}

\subsection{Full Optimization Tables}

\subsubsection{Preliminary Method with $m C P B A$ as Oxidant}

Table S1: Preliminary aotimization of the one-pot synthesis of $10 \mathrm{H}$-dibenzo[b,e]iodinin-5-ium trifluoromethanesulfonate $7 a$.<smiles>OCc1ccccc1I</smiles>

$5 a$
1) $\mathrm{TfOH}$, benzene (6a) temp.

2) $m$ CPBA, temp. solvent, time, temp

$7 a$

\begin{tabular}{|c|c|c|c|c|c|c|}
\hline$\#^{\mathrm{a}}$ & $\begin{array}{l}\text { TfOH } \\
\text { (Eq.) }\end{array}$ & $\begin{array}{c}m \text { mCPBA } \\
\text { (Eq.) }\end{array}$ & $\begin{array}{l}\text { Solv. } \\
(0.1 \mathrm{M})\end{array}$ & $\begin{array}{c}\mathrm{T} /{ }^{\circ} \mathrm{C} \\
\text { Step } 1 / \text { Step } 2\end{array}$ & $\begin{array}{l}\text { Time } \\
\text { Step } 2\end{array}$ & $\begin{array}{c}\text { Yield (Purity }) \\
\quad / \%(\%)\end{array}$ \\
\hline 1 & 2 & 1.1 & 1,2-DCE & $80 / 0$ & 2 & $79(75-80)$ \\
\hline 2 & 3 & 1.1 & 1,2-DCE & rt / 0 & 2 & $79(75-80)$ \\
\hline $3^{c}$ & 3 & 1.1 & DCM & $\mathrm{rt} / 0$ & 2 & $68(80-85)$ \\
\hline $4^{\mathrm{c}, \mathrm{d}}$ & 3 & 1.1 & DCM & $\mathrm{rt} / 0$ & --- & -- \\
\hline $5^{c}$ & 3 & 1.1 & DCM & 0 / 0 & 2 & $76(80-85)$ \\
\hline $6^{c}$ & 3 & 1.5 & DCM & $0 / 0$ & 1.5 & $75(80-85)$ \\
\hline $7^{e}$ & 3 & 1.5 & DCM & $0 / 0$ & 1.5 & $85(80-85)$ \\
\hline $8^{e, f}$ & 3 & 1.5 & DCM & $0 / 0$ & 1.5 & 64 (>95) \\
\hline $9^{e, f}$ & 3 & 1.5 & DCM & $0 / 0-40$ & 1 & 65 (> 95) \\
\hline $10^{e, f}$ & 2.5 & 1.5 & DCM & $0 / 0-40$ & 1 & 66 (>95) \\
\hline $11^{e, f}$ & 3.5 & 1.5 & DCM & $0 / 0-40$ & 1 & 60 (>95) \\
\hline $12^{\mathrm{e}, \mathrm{f}, \mathrm{g}}$ & 2.5 & 1.5 & $\mathrm{DCM}$ & $0 / 0-40$ & 1 & $73(>95)$ \\
\hline
\end{tabular}

${ }^{a}$ All reactions were performed on a $0.1 \mathrm{mmol}$ scale with benzene (10 eq.). in the given solvent $(0.5 \mathrm{~mL})$ were used in each of the reaction steps.

${ }^{\mathrm{b}}$ Approximate purity as molar fraction determined via $1 \mathrm{H}-\mathrm{NMR}$ spectroscopy.

c Dropwise addition of $m C P B A$ in $D C M(0.5 \mathrm{~mL})$ via syringe.

${ }^{d}$ First addition of $m C P B A$ in DCM and then TfOH in DCM.

e Dropwise addition of $m C P B A$ in $0.5 \mathrm{~mL} \mathrm{DCM}$ via syringe-pump (rate: $50 \mu \mathrm{L} / \mathrm{min}$ ).

${ }^{f}$ Purification of the crude product via filtration over silica and washing with DCM/MeOH 25:1.

${ }^{\mathrm{g}}$ Dropwise addition of the benzylic alcohol in DCM $(0.5 \mathrm{~mL})$ to a solution of benzene in DCM $(0.5 \mathrm{~mL})$ via syringe pump (rate: $50 \mu \mathrm{L} / \mathrm{min}$ ). 


\subsubsection{Preliminary Test Reactions for the Oxidation of 9a}

Table S2: Optimization for the oxidation reaction of $9 a$ for the synthesis of 10H-dibenzo[b,e]iodinin-5-ium trifluoromethanesulfonate $7 a$.

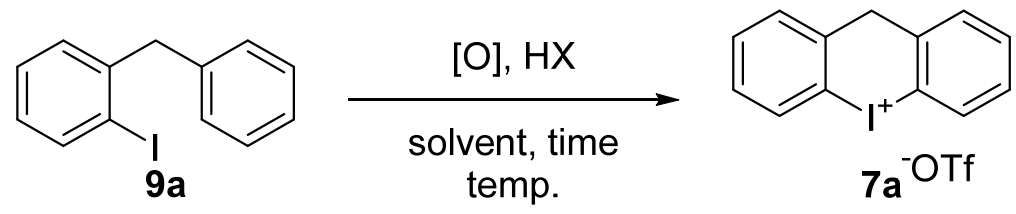

\begin{tabular}{|c|c|c|c|c|c|c|}
\hline$\#^{\mathrm{a}}$ & $\begin{array}{l}\text { [O] } \\
\text { (Eq.) }\end{array}$ & $\begin{array}{l}\mathrm{HX} \\
\text { (Eq.) }\end{array}$ & Solv. (c) & $\mathrm{T} /{ }^{\circ} \mathrm{C}$ & $\begin{array}{c}\text { Time } \\
/ \mathrm{h}\end{array}$ & $\begin{array}{c}\text { Yield }^{\mathbf{b}} \\
/ \%\end{array}$ \\
\hline 1 & mCPBA (1.2) & TfOH (2.5) & DCM (0.1 M) & $\mathrm{rt}$ & 18 & 71 \\
\hline 2 & Oxone $^{\circledR}(0.6)$ & TfOH (2.5) & DCM (0.1 M) & $\mathrm{rt}$ & 18 & 57 \\
\hline 3 & $\mathrm{~K}_{2} \mathrm{~S}_{2} \mathrm{O}_{8}(1.2)$ & TfOH (2.5) & DCM (0.1 M) & $\mathrm{rt}$ & 18 & 28 \\
\hline 4 & $\mathrm{NaBO}_{3} \cdot 4 \mathrm{H}_{2} \mathrm{O}(1.2)$ & TfOH (2.5) & DCM (0.1 M) & $\mathrm{rt}$ & 18 & 8.3 \\
\hline 5 & Oxone $^{\circledR}(0.6)$ & TfOH (2.5) & DCM (0.1 M) & $0-r t$ & 18 & 20 \\
\hline 6 & Oxone $^{\circledR}(1)$ & TfOH (2.5) & DCM (0.1 M) & $0-r t$ & 18 & 39 \\
\hline 7 & Oxone $^{\circledR}(1.5)$ & TfOH (2.5) & DCM (0.1 M) & $0-r t$ & 18 & 26 \\
\hline 8 & Oxone $^{\circledR}(1)$ & TfOH (2.5) & DCM/ACN (0.1 M) & $\mathrm{rt}$ & 120 & 56 \\
\hline 9 & Oxone $^{\circledR}(2)$ & TfOH (2.5) & DCM/ACN (0.1 M) & $\mathrm{rt}$ & 18 & Mult. Prods. \\
\hline 10 & Oxone $^{\circledR}(5)$ & TfOH (2.5) & DCM/ACN (0.1 M) & $\mathrm{rt}$ & 18 & Mult. Prods. \\
\hline 11 & Oxone $^{\circledR}(10)$ & TfOH (2.5) & DCM/ACN (0.1 M) & $\mathrm{rt}$ & 18 & 15 \\
\hline 12 & Oxone ${ }^{\circledR}(1)$ & TfOH (3.5) & DCM/ACN (0.1 M) & $\mathrm{rt}$ & 18 & 75 \\
\hline 13 & Oxone ${ }^{\circledR}(1)$ & TfOH (5) & DCM/ACN (0.1 M) & $\mathrm{rt}$ & 18 & 91 \\
\hline 14 & Oxone $^{\circledR}(2)$ & TfOH (5) & DCM/ACN (0.1 M) & $\mathrm{rt}$ & 18 & 83 \\
\hline 15 & Oxone $^{\circledR}(1)$ & $\mathrm{H}_{2} \mathrm{SO}_{4}(5)$ & DCM/ACN (0.1 M) & $\mathrm{rt}$ & 72 & $<10$ \\
\hline 16 & Oxone $^{\circledR}(1)$ & $\mathrm{HBF}_{4}(5)$ & DCM/ACN (0.1 M) & $\mathrm{rt}$ & 72 & 30 \\
\hline 17 & Oxone $^{\circledR}(1)$ & $\mathrm{HBF}_{4}(5), \mathrm{H}_{2} \mathrm{SO}_{4}(5)$ & DCM/ACN (0.1 M) & $\mathrm{rt}$ & 72 & 85 \\
\hline 18 & Oxone $^{\circledR}(1)$ & $\mathrm{TfOH}(5), \mathrm{H}_{2} \mathrm{SO}_{4}(5)$ & DCM/ACN (0.1 M) & rt & 2 & 92 \\
\hline 19 & Oxone $^{\circledR}(1)$ & $\mathrm{HBF}_{4}(5), \mathrm{H}_{2} \mathrm{SO}_{4}(5)$ & DCM/ACN $(0.1 \mathrm{M})^{\mathrm{C}}$ & $\mathrm{rt}-40$ & 24 & 4 \\
\hline 20 & Oxone $^{\circledR}(1)$ & $\mathrm{TfOH}(5), \mathrm{H}_{2} \mathrm{SO}_{4}(5)$ & $\mathrm{DCM} / \mathrm{ACN}(0.1 \mathrm{M})^{\mathrm{C}}$ & $\mathrm{rt}$ & $1-2$ & 87 \\
\hline
\end{tabular}

${ }^{a}$ All reactions were performed on a $0.2 \mathrm{mmol}$ scale

${ }^{\mathrm{b}}$ Yield determined after isolation and purification of the product $7 \mathrm{a}$.

${ }^{c} 5 \%$ of TFE were added. 


\subsubsection{Final Method for Primary Alcohols with Oxone ${ }^{\circledR}$ as Oxidant}

Table S3: Final optimization of the one-pot synthesis of $10 \mathrm{H}$-dibenzo[b,e]iodinin-5-ium trifluoromethanesulfonate $7 a$
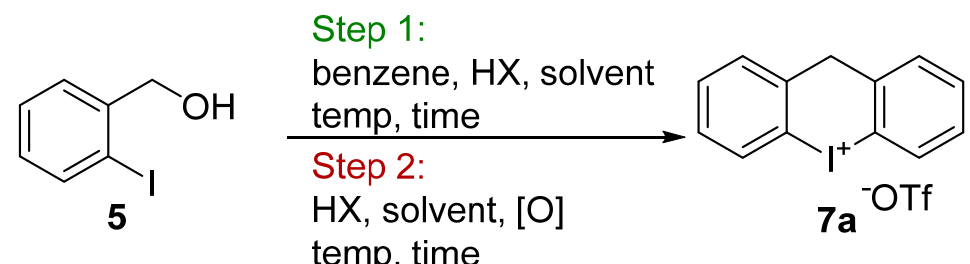

\begin{tabular}{|c|c|c|c|c|c|}
\hline$\#^{\mathrm{a}}$ & $\begin{array}{l}\text { Benzene } \\
\text { (Eq.) }\end{array}$ & $\begin{array}{c}\mathrm{HX} \\
\text { (Eq.) }\end{array}$ & $\begin{array}{l}\text { Solv. } \\
\text { (c) }\end{array}$ & $\begin{array}{c}\text { Time } \\
\text { Step } 1 \text { / Step } 2\end{array}$ & $\begin{array}{l}\text { Yield } \\
/ \%\end{array}$ \\
\hline 1 & benzene (3) & $\mathrm{TfOH}(5), \mathrm{H}_{2} \mathrm{SO}_{4}(5)$ & DCM/ACN (0.1 M) & $5 \mathrm{~min} / 1.5 \mathrm{~h}$ & (39) \\
\hline 2 & benzene (5) & $\mathrm{TfOH}(5), \mathrm{H}_{2} \mathrm{SO}_{4}(5)$ & DCM/ACN (0.1 M) & $5 \mathrm{~min} / 1.5 \mathrm{~h}$ & 77 \\
\hline 3 & benzene (10) & $\mathrm{TfOH}(5), \mathrm{H}_{2} \mathrm{SO}_{4}(5)$ & DCM/ACN (0.1 M) & $5 \mathrm{~min} / 1.5 \mathrm{~h}$ & 71 \\
\hline $4^{b}$ & benzene (10) & $(5), \mathrm{H}_{2} \mathrm{SO}_{4}(5)$ & DCN /ACN (0.1 M) & $5 \mathrm{~min} / 1.5 \mathrm{~h}$ & 89 \\
\hline $5^{b, c}$ & benzene (10) & $\mathrm{TfOH}(5), \mathrm{H}_{2} \mathrm{SO}_{4}$ (5) & DCM (0.1 M) & $5 \mathrm{~min} / 24 \mathrm{~h}$ & $<10$ \\
\hline $6^{b, d}$ & benzene (10) & $\mathrm{TfOH}(5), \mathrm{H}_{2} \mathrm{SO}_{4}(5)$ & ACN (0.1 M) & $360 \mathrm{~min} /$--- & 0 \\
\hline $7^{b}$ & benzene (10) & $\mathrm{TfOH}(5), \mathrm{H}_{2} \mathrm{SO}_{4}(5)$ & $\begin{array}{c}\text { DCM/ACN/ } \\
\text { HFIPe (0.1 M) }\end{array}$ & $5 \mathrm{~min} / 1 \mathrm{~h}$ & 89 \\
\hline $8^{b}$ & benzene (10) & $\mathrm{TfOH}(3), \mathrm{H}_{2} \mathrm{SO}_{4}(5)$ & DCM/ACN (0.1 M) & $5 \mathrm{~min} / 4 \mathrm{~h}$ & 75 \\
\hline $9^{b}$ & benzene (10) & $\mathrm{TfOH}(2), \mathrm{H}_{2} \mathrm{SO}_{4}$ (5) & DCM/ACN (0.1 M) & $5 \mathrm{~min} / 4 \mathrm{~h}$ & 53 \\
\hline $10^{b}$ & benzene (10) & $\mathrm{TfOH}(\mathbf{1}), \mathrm{H}_{2} \mathrm{SO}_{4}(5)$ & DCM/ACN (0.1 M) & $3.5 \mathrm{~h} / 12 \mathrm{~h}$ & 50 \\
\hline $11^{\mathrm{b}}$ & benzene (10) & $\mathrm{TfOH}$ (3), $\mathrm{H}_{2} \mathrm{SO}_{4}$ (7.5) & DCM/ACN (0.1 M) & $5 \mathrm{~min} / 4 \mathrm{~h}$ & 81 \\
\hline $12^{\mathrm{b}}$ & benzene (10) & $\mathrm{TfOH}(\mathbf{3}), \mathrm{H}_{2} \mathrm{SO}_{4}$ (10) & DCM/ACN (0.1 M) & $5 \mathrm{~min} / 4 \mathrm{~h}$ & 80 \\
\hline $13^{b}$ & benzene (10) & $\mathrm{TfOH}$ (3), $\mathrm{H}_{2} \mathrm{SO}_{4}$ (7.5) & DCM/ACN (0.1 M) & $5 \mathrm{~min} / 2 \mathrm{~h}$ & (71) \\
\hline $14^{\mathrm{b}}$ & benzene (10) & $\mathrm{TfOH}$ (5), $\mathrm{H}_{2} \mathrm{SO}_{4}$ (7.5) & DCM/ACN (0.1 M) & $5 \mathrm{~min} / 1.5 \mathrm{~h}$ & 85 \\
\hline $15^{b}$ & benzene (28) & $\mathrm{TfOH}(5), \mathrm{H}_{2} \mathrm{SO}_{4}(5)$ & DCM/ACN (0.1 M) & $5 \mathrm{~min} / 2 \mathrm{~h}$ & 90 \\
\hline $16^{b}$ & benzene (5) & $\mathrm{TfOH}(5), \mathrm{H}_{2} \mathrm{SO}_{4}(5)$ & DCM/ACN (0.1 M) & $5 \mathrm{~min} / 2 \mathrm{~h}$ & $87^{f}$ \\
\hline $17^{b}$ & benzene (3) & $\mathrm{TfOH}(5), \mathrm{H}_{2} \mathrm{SO}_{4}$ (5) & DCM/ACN (0.1 M) & $5 \mathrm{~min} / 1.3 \mathrm{~h}$ & $92^{f}$ \\
\hline $18^{b, g}$ & benzene (28) & $\mathrm{TfOH}(5), \mathrm{H}_{2} \mathrm{SO}_{4}$ (5) & DCM/ACN (0.1 M) & $5 \mathrm{~min} / 2 \mathrm{~h}$ & 80 \\
\hline $19^{b, h}$ & benzene (10) & $\mathrm{TfOH}(5), \mathrm{H}_{2} \mathrm{SO}_{4}(5)$ & DCM/ACN (0.1 M) & $5 \mathrm{~min} / 2 \mathrm{~h}$ & 93 \\
\hline
\end{tabular}

Yields in parentheses indicate that the purity of the product was below $90 \%$

${ }^{a}$ All reactions were performed on a $0.2 \mathrm{mmol}$ scale with Oxone $\left(2 \mathrm{KHSO}_{5} \cdot \mathrm{KHSO}_{4} \cdot \mathrm{K}_{2} \mathrm{SO}_{4}, 1\right.$ eq.) in a tightly closed screw cap vial. Different colours in the table indicate that reagents were used in different steps of the reaction. Step 1: Acid $A$ in solvent A $(1 \mathrm{~mL})$ with Nuc stirred at $0{ }^{\circ} \mathrm{C}$. Step 2: Solvent B $(1 \mathrm{~mL})$ added, Acid B and Oxone added at $0{ }^{\circ} \mathrm{C}$.

${ }^{\mathrm{b}} \mathrm{o}$-lodobenzylalcohol was added dropwise in solvent $(0.5 \mathrm{~mL})$ to a solution of Nuc in solvent $(0.5 \mathrm{~mL})$.

${ }^{c}$ No difference if $\mathrm{H}_{2} \mathrm{SO}_{4}$ is added before or after Oxone, very messy reaction in step 2.

d No reaction in step 1.

e HFIP $(0.2 \mathrm{~mL})$ in second step.

${ }^{f}$ Purity between 90 and $95 \%$.

g Open flask.

h $5 \mathrm{mmol}$ scale. 


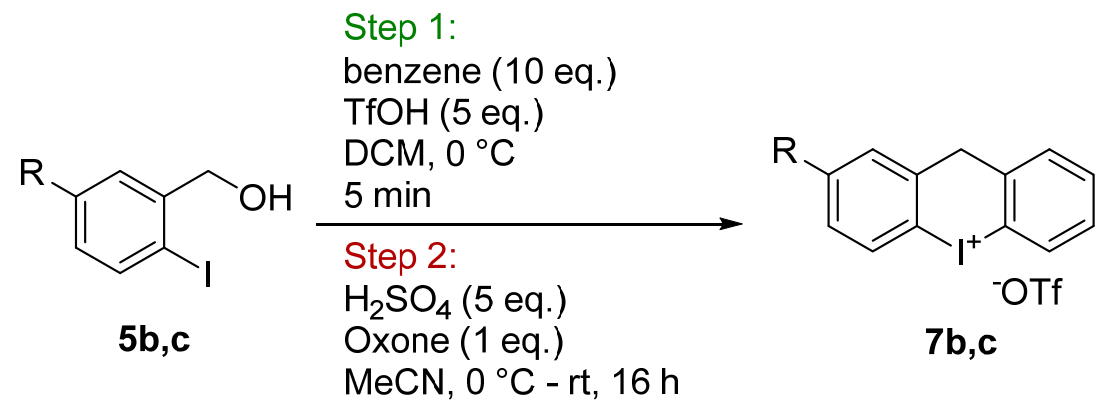

\begin{tabular}{|c|c|c|c|}
\hline$\#^{\mathrm{a}}$ & $\mathbf{R}$ & Deviation from standard condition & $\begin{array}{c}\text { Yield } \\
/ \%\end{array}$ \\
\hline 1 & $\mathrm{Br}(5 \mathrm{~b})$ & --- & 57 \\
\hline 2 & $\mathrm{Br}$ & $\mathrm{No} \mathrm{H}_{2} \mathrm{SO}_{4}$ in step 2 & 52 \\
\hline 3 & $\mathrm{Br}$ & Addition of $0.2 \mathrm{~mL}$ HFIP in step 2 & 85 \\
\hline 4 & $\mathrm{Br}$ & TFA instead of MeCN & 50 \\
\hline 5 & $\mathrm{Br}$ & $2 \mathrm{~mL} \mathrm{MeCN}$ in step 2 & 49 \\
\hline 6 & $\mathrm{Br}$ & Use of Pestled Oxone & 78 \\
\hline 7 & $\mathrm{Br}$ & DCM instead of MeCN & $<5$ \\
\hline 8 & $\mathrm{Br}$ & HFIP + Pestled Oxone & 83 \\
\hline $9^{b}$ & $\mathrm{NO}_{2}(5 \mathrm{c})$ & --- & $40-50$ \\
\hline 10 & $\mathrm{NO}_{2}$ & $40^{\circ} \mathrm{C}$ in step $2,2^{\text {nd }}$ eq. of Oxone after $16 \mathrm{~h}$ & $35-45$ \\
\hline 11 & $\mathrm{NO}_{2}$ & $40^{\circ} \mathrm{C}, 2$ eq. Oxone in step 2 & 27 \\
\hline 12 & $\mathrm{NO}_{2}$ & \# 10 with 7.5 eq. $\mathrm{H}_{2} \mathrm{SO}_{4}$ & $30-40$ \\
\hline 13 & $\mathrm{NO}_{2}$ & $\# 10$ with 10 eq. $\mathrm{H}_{2} \mathrm{SO}_{4}$ & 27 \\
\hline 14 & $\mathrm{NO}_{2}$ & $2^{\text {nd }}$ eq. of Oxone and 5 eq. of $\mathrm{H}_{2} \mathrm{SO}_{4}$ after $16 \mathrm{~h}$ & 84 \\
\hline
\end{tabular}

a Standard conditions: $0.2 \mathrm{mmol}$ of alcohol 5 in DCM (1 mL) with benzene (10 eq.). Addition of TfOH (5 eq.) at $0{ }^{\circ} \mathrm{C}$, stirring for $2 \mathrm{~h}\left(\mathrm{R}=\mathrm{Br}\right.$ ) or $5 \mathrm{~h}\left(\mathrm{R}=\mathrm{NO}_{2}\right)$, addition of $\mathrm{MeCN}(1 \mathrm{~mL}), \mathrm{H}_{2} \mathrm{SO}_{4}$ (5 eq.) and Oxone (1 eq.). Stirring for $16 \mathrm{~h}$ at rt.

$\mathrm{b}$ The standard conditions for $\mathrm{R}=\mathrm{NO}_{2}$ are identical to those in (a) with HFIP $(0.2 \mathrm{~mL})$ in step 2 . 


\subsubsection{Preliminary Method for Secondary Alcohols with $m$ CPBA as Oxidant}

Table S5: Reoptimization for secondary benzylic alcohols with toluene.
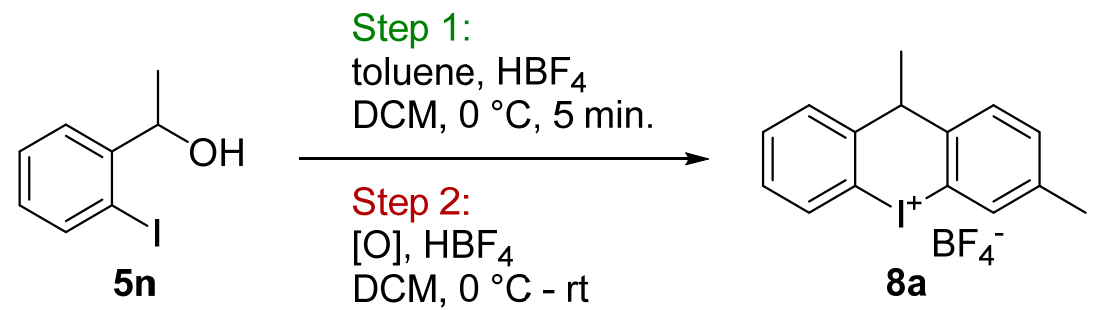

\begin{tabular}{|c|c|c|c|c|c|}
\hline$\#^{\mathrm{a}}$ & PhMe (eq.) & $\begin{array}{l}\mathrm{HBF}_{4} \text { (eq.) } \\
1^{\text {st }} / 2^{\text {nd }} \text { step }\end{array}$ & [0] (eq.) & $\begin{array}{l}\text { Deviation from } \\
\text { standard condition }\end{array}$ & Yield / \% \\
\hline $1^{b}$ & 5 & $1 / 2$ & $m$ CPBA (4) & & $97 \%$ \\
\hline 2 & 5 & $1 / 2$ & $m C P B A(4)$ & & $67 \%$ \\
\hline 3 & 5 & $1 / 2$ & $m C P B A(2)$ & & $83 \%$ \\
\hline $4^{c}$ & 5 & $1 / 2$ & $m C P B A(4)$ & & $53 \%$ \\
\hline $5^{c}$ & 5 & $1 / 5$ & $m C P B A(1)$ & & $58 \%$ \\
\hline $6^{c}$ & 5 & $1 / 5$ & $m C P B A(2)$ & & $70 \%$ \\
\hline $7^{c}$ & 5 & $3 / 2$ & $m$ CPBA (2) & & $81 \%$ \\
\hline $8^{c}$ & 5 & $5 / 5$ & $m C P B A(2)$ & & $82 \%$ \\
\hline $9^{b c}$ & 3 & $3 / 2$ & $m$ СРВA (1.5) & & $88 \%$ \\
\hline $10^{c}$ & 3 & $3 / 2$ & $m$ СРВA (1.5) & & $78 \%$ \\
\hline $11^{\mathrm{c}}$ & 3 & $3 / 3$ & $m$ CPBA (1.5) & & $81 \%$ \\
\hline $12^{\mathrm{c}}$ & 3 & $5 / 0$ & $m C P B A(1)$ & & $78 \%$ \\
\hline $13^{c}$ & 3 & $5 / 0$ & $m$ CPBA (2) & & $79 \%$ \\
\hline $14^{\mathrm{bc}}$ & 3 & $3 / 2$ & Oxone (1) & addition of $\mathrm{H}_{2} \mathrm{SO}_{4}$ (2 eq.) & --- \\
\hline $15^{\mathrm{bc}}$ & 3 & $3 / 5$ & Oxone (1) & addition of $\mathrm{H}_{2} \mathrm{SO}_{4}$ (10 eq.) & $50 \%$ \\
\hline $16^{\mathrm{b}}$ & 3 & $3 / 5$ & Oxone (1) & $\begin{array}{l}\mathrm{CH}_{3} \mathrm{CN} \text { instead of DCM, } \\
\text { addition of } \mathrm{H}_{2} \mathrm{SO}_{4} \text { (10 eq.) }\end{array}$ & $22 \%$ \\
\hline $17^{\mathrm{bc}}$ & 3 & $3 / 0$ & Oxone (2) & addition of $\mathrm{H}_{2} \mathrm{SO}_{4}$ (15 eq.) & $8 \%$ \\
\hline
\end{tabular}

a Standard conditions: $0.2 \mathrm{mmol}$ of $\mathbf{5 n}$ in $1 \mathrm{~mL} \mathrm{DCM}$ with toluene (5 eq.). Addition of $\mathrm{HBF}_{4} \cdot \mathrm{OEt}_{2}\left(1\right.$ eq.) at $0{ }^{\circ} \mathrm{C}$, stirring till completion, addition of $1 \mathrm{~mL} \mathrm{MeCN}, m C P B A$, stirring for $10 \mathrm{~min}$, addition of $\mathrm{HBF}_{4} \cdot \mathrm{OEt}_{2}$ (2 eq.), stirring till completion at rt. ${ }^{\mathrm{b}} 0.1 \mathrm{mmol}$ scale.

${ }^{\mathrm{c}} \mathrm{MeCN}$ in the $2^{\text {nd }}$ step. 


\section{Preparation of Starting Materials}

\subsection{General Procedures for the Synthesis of the Starting Materials}<smiles>NC1=CC=[R]#CC=C1C(=O)O</smiles><smiles>CCCCCCC</smiles><smiles></smiles><smiles>CCCCCC</smiles><smiles>OCc1ccccc1I</smiles>

Primary Benzyl Alcohols $5 a-5 m$<smiles>[R]C(O)c1ccccc1I</smiles><smiles>[3H]I</smiles>

Secondary Benzyl Alcohols $5 n-5 p$<smiles></smiles>

S2
Derivatizations<smiles>[X]Cc1ccccc1I</smiles>

Primary Benzyl Alcohol Derivatives $10-13$

Scheme S1: General scheme for the synthesis of primary and secondary benzyl alcohol derivatives.

3.1.1 Sandmeyer-type lodination of Anthranilic acids (GP1a)

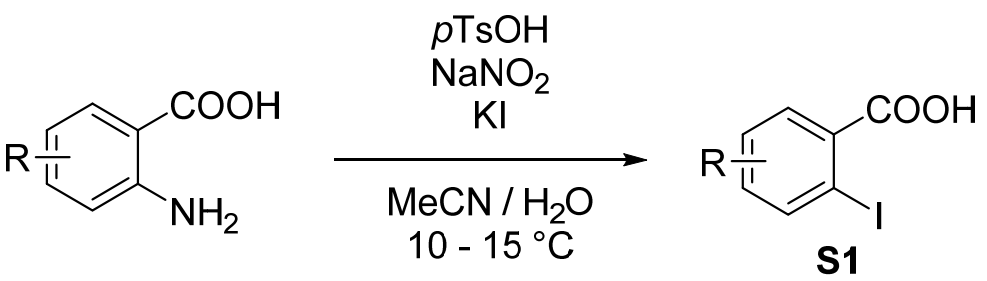

In modification of a reported procedure, ${ }^{[2]}$ the anthranilic acid derivative (10.0 mmol, 1.00 eq.) and $\mathrm{TsOH} \cdot \mathrm{H}_{2} \mathrm{O}(5.71 \mathrm{~g}, 30.0 \mathrm{mmol}, 3.00 \mathrm{eq}$.) were suspended in $\mathrm{MeCN}(80 \mathrm{~mL})$. The mixture was cooled to $10-15{ }^{\circ} \mathrm{C}$. A solution of $\mathrm{NaNO}_{2}(1.38 \mathrm{~g}, 20.0 \mathrm{mmol}, 2.00$ eq.) and $\mathrm{KI}(4.15 \mathrm{~g}, 25.0 \mathrm{mmol}, 2.50$ eq.) in $\mathrm{H}_{2} \mathrm{O}(15 \mathrm{~mL})$ was added dropwise over $1 \mathrm{~h}$. After complete addition, the resulting dark coloured mixture stirred for another $1 \mathrm{~h}$ and then $1 \mathrm{~N} \mathrm{HCl}(50 \mathrm{~mL})$ and EtOAc $(100 \mathrm{~mL})$ were added. The phases were separated and the aqueous phases was extracted with EtOAc $(2 \times 50 \mathrm{~mL})$. The combined organic phases were washed with water $(50 \mathrm{~mL}), \mathrm{Na}_{2} \mathrm{~S}_{2} \mathrm{O}_{3}(10 \% \mathrm{w} / \mathrm{w}, 50 \mathrm{~mL})$ and brine $(20 \mathrm{~mL})$, dried over $\mathrm{Na}_{2} \mathrm{SO}_{4}$ and concentrated under reduced pressure. The residue was either purified by filtration over silica or by column chromatography.

\subsubsection{Sandmeyer-type lodination of Anthranilic acids (GP1b)}<smiles>Nc1cc[R]#cc1C(=O)O</smiles>

1) $\mathrm{NaNO}_{2}$, aq. $\mathrm{H}_{2} \mathrm{SO}_{4}$ $\mathrm{MeOH}, \mathrm{O}^{\circ} \mathrm{C}$

2) $\mathrm{KI}$ $\mathrm{H}_{2} \mathrm{O}, 60^{\circ} \mathrm{C}$<smiles>O=C(O)c1cc[R]#cc1I</smiles> 
In modification of a reported procedure, ${ }^{[3]}$ the anthranilic acid derivative $(10.0 \mathrm{mmol}, 1.00$ eq.) was suspended in aqueous $\mathrm{H}_{2} \mathrm{SO}_{4}\left(15 \mathrm{~mL}, 1: 2 \mathrm{v} / \mathrm{v}\right.$ ) at $0{ }^{\circ} \mathrm{C}$ and $\mathrm{MeOH}$ ( 5 to $10 \mathrm{~mL}$ ) was added until complete dissolution of the substrate. Then a solution of $\mathrm{NaNO}_{2}\left(759 \mathrm{mg}, 11.0 \mathrm{mmol}, 1.10\right.$ eq.) in $\mathrm{H}_{2} \mathrm{O}(2 \mathrm{~mL})$ was added dropwise over $30 \mathrm{~min}$. while maintaining a temperature between 0 and $5{ }^{\circ} \mathrm{C}$. After complete addition the solution was stirred for further $60 \mathrm{~min}$ at $0{ }^{\circ} \mathrm{C}$. Then the diazonium salt solution was added to a solution of $\mathrm{KI}(8.30 \mathrm{~g}, 50.0 \mathrm{mmol}, 5.00$ eq. $)$ in $\mathrm{H}_{2} \mathrm{O}(10 \mathrm{~mL})$ at $60{ }^{\circ} \mathrm{C}$ over a period of $5 \mathrm{~min}$. and stirred at that temperature for $1 \mathrm{~h}$. Then $1 \mathrm{~N} \mathrm{HCl}(50 \mathrm{~mL})$ and EtOAc $(100 \mathrm{~mL})$ were added. The phases were separated and the aqueous phases was extracted with EtOAc $(2 \times 50 \mathrm{~mL})$. The combined organic phases were washed with water $(50 \mathrm{~mL}), \mathrm{Na}_{2} \mathrm{~S}_{2} \mathrm{O}_{3}(10 \% \mathrm{w} / \mathrm{w}, 50 \mathrm{~mL})$ and brine $(20 \mathrm{~mL})$, dried over $\mathrm{Na}_{2} \mathrm{SO}_{4}$ and concentrated under reduced pressure. The residue was either purified by filtration over silica or by column chromatography.

\subsubsection{Borane-mediated Reduction of Carboxylic acids (GP2)}

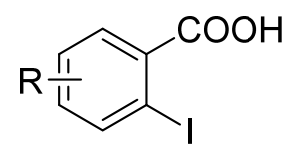

S1

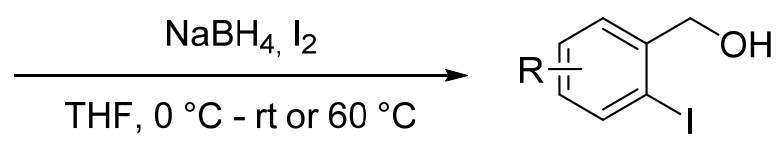

5

In modification of a reported procedure, ${ }^{[4]}$ the 0 -iodobenzoic acid derivative (S1, 1.00 eq.) was dissolved in dry THF $(0.25 \mathrm{M})$. After cooling to $0{ }^{\circ} \mathrm{C}, \mathrm{NaBH}_{4}(2.90$ eq.) was added portion wise. To the resulting mixture $\mathrm{I}_{2}(0.75$ eq.) in THF $(0.25 \mathrm{M})$ was slowly added over $1 \mathrm{~h}$. After the addition, the mixture was allowed to warm to $\mathrm{rt}$ and stirred overnight. After completion of the reaction, the mixture was cooled to $0{ }^{\circ} \mathrm{C}$ and water $(1 \mathrm{~mL} / \mathrm{mmol})$ was carefully added. Afterwards, $3 \mathrm{~N} \mathrm{HCl}$ was slowly added until the solution was at $\mathrm{pH} 2-3$. The resulting mixture was diluted with water $(5 \mathrm{~mL} / \mathrm{mmol})$ and extracted with $\mathrm{Et}_{2} \mathrm{O}(3 \times 20 \mathrm{~mL} / \mathrm{mmol})$. The combined organic phases were washed with $1 \mathrm{M} \mathrm{Na}_{2} \mathrm{CO}_{3}(2 \times$ $20 \mathrm{~mL} / \mathrm{mmol})$ and brine $(20 \mathrm{~mL} / \mathrm{mmol})$, dried over $\mathrm{Na}_{2} \mathrm{SO}_{4}$ and concentrated under reduced pressure to either give the desired product that could be purified (if necessary) by recrystallisation from cyclohexane or via a filtration column over silica in cyclohexane/ethyl acetate 4:1.

\subsection{Synthesis of Starting Materials}

\subsubsection{Primary Benzyl Alcohols}

The $o$-iodobenzoic acid derivatives S1a, S1d and S1e were commercially available and have been used as received for the synthesis of the corresponding alcohols.

\section{(2-lodophenyl)methanol (5a)}

Following GP2, the reaction of $o$-iodobenzoic acid (S1a, $9.6 \mathrm{~g}, 200 \mathrm{mmol}$ ) with $\mathrm{NaBH}_{4} \quad(21.9 \mathrm{~g}, \quad 580 \mathrm{mmol})$ and $\mathrm{I}_{2} \quad(38.1 \mathrm{~g}, 150 \mathrm{mmol})$ gave (2iodophenyl)methanol (5a, $46.8 \mathrm{~g}, 200 \mathrm{mmol}$, quant.) as a colourless solid

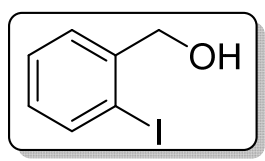
without further purification.

${ }^{1} \mathrm{H}-\mathrm{NMR}\left(601 \mathrm{MHz}, \mathrm{CDCl}_{3}\right) \delta=7.83(\mathrm{dd}, J=7.8,1.2 \mathrm{~Hz}, 1 \mathrm{H}), 7.46$ (dd, $\left.J=7.6,1.7 \mathrm{~Hz}, 1 \mathrm{H}\right), 7.37$ (td, $J=$ 7.5, $1.2 \mathrm{~Hz}, 1 \mathrm{H}), 7.00(\mathrm{td}, J=7.6,1.8 \mathrm{~Hz}, 1 \mathrm{H}), 4.68(\mathrm{~d}, J=6.3 \mathrm{~Hz}, 2 \mathrm{H}), 2.00(\mathrm{t}, J=6.3 \mathrm{~Hz}, 1 \mathrm{H}) .{ }^{13} \mathrm{C}-\mathrm{NMR}$ 
(151 MHz, CDCl3) $\delta=142.9,139.5,129.6,128.8,128.7,97.7,69.6$. FTIR (ATR, neat) $\tilde{v}=3262,3061$, 2887, 2843, 1434, 1321, 1195, 1033, 1009, 738. MS (El, 70 eV) $\mathrm{m} / z=234.0[\mathrm{M}]^{+}$Mp $T=91$. The analytical data is in accordance with literature data. ${ }^{[5]}$

\section{(5-Bromo-2-iodophenyl)methanol (5b)}

o-lodobenzoic acid (4.96 g, $20.0 \mathrm{mmol}, 1.00$ eq.) and $\mathrm{N}$-bromosuccinmide ( $4.45 \mathrm{~g}, 25.0 \mathrm{mmol}, 1.25$ eq.) were suspended in $\mathrm{H}_{2} \mathrm{SO}_{4}(50 \mathrm{~mL}$ ) and stirred for $2 \mathrm{~h}$. The mixture was poured on ice $(150 \mathrm{~g})$. The resulting solid was collected by filtration and recrystallized from EtOH/ $\mathrm{H}_{2} \mathrm{O}(1: 1, \mathrm{v} / \mathrm{v})$ to obtain 5-bromo-2iodobenzoic acid (S1b, $5.21 \mathrm{~g}, 15.9 \mathrm{mmol}, 80 \%$ ) as a colourless solid.

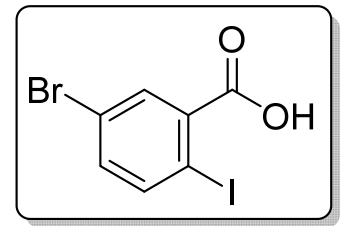

${ }^{1} \mathrm{H}-\mathrm{NMR}\left(360 \mathrm{MHz}, \mathrm{CDCl}_{3}\right) \delta=8.15(\mathrm{~d}, J=2.4 \mathrm{~Hz}, 1 \mathrm{H}), 7.91(\mathrm{~d}, J=8.4 \mathrm{~Hz}, 1 \mathrm{H}), 7.34(\mathrm{dd}, J=8.4,2.4 \mathrm{~Hz}$, 1H). ${ }^{13}$ C-NMR (91 MHz, $\mathrm{CDCl}_{3}$ ) $\delta=169.9,143.2,136.6,135.0,134.5,122.3,92.8$. FTIR (ATR, neat) $\tilde{v}=$ 3079, 1702, 1668, 1459, 1294, 1248, 1096, 1015, 898, 816, 776. HRMS (ESI-, MeOH) $m / z=324.82642$. Calculated for $\mathrm{C}_{7} \mathrm{H}_{3} \mathrm{BrlO}_{2}: \mathrm{m} / \mathrm{z}=324.83666$. $\mathrm{Mp} T=159-161$. The analytical data is in accordance with literature data. ${ }^{[6]}$

Following GP2, the reaction of 5-bromo-2-iodobenzoic acid (S1b, $1.63 \mathrm{~g}$, $5.00 \mathrm{mmol}$ ) with $\mathrm{NaBH}_{4}\left(549 \mathrm{mg}, 14.5 \mathrm{mmol}\right.$ ) and $\mathrm{I}_{2}$ (952 mg, $3.75 \mathrm{mmol}$ ) gave after filtration over silica (cyclohexane/ethyl acetate 7:1) (5-bromo-2iodophenyl)methanol (5b, $1.53 \mathrm{~g}, 4.87 \mathrm{mmol}, 97 \%)$ as a colourless solid.

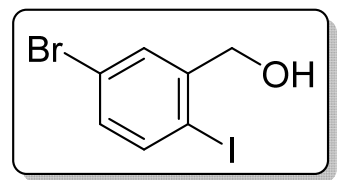

${ }^{1} \mathrm{H}-\mathrm{NMR}\left(360 \mathrm{MHz}, \mathrm{CDCl}_{3}\right) \delta=7.63(\mathrm{~d}, J=8.3 \mathrm{~Hz}, 1 \mathrm{H}), 7.61(\mathrm{~d}, J=1.9 \mathrm{~Hz}, 1 \mathrm{H}), 7.12(\mathrm{dd}, J=8.3,2.0 \mathrm{~Hz}$, $1 \mathrm{H}), 4.61(\mathrm{~d}, J=6.0 \mathrm{~Hz}, 2 \mathrm{H}), 2.23(\mathrm{t}, J=6.0,6.0 \mathrm{~Hz}, 1 \mathrm{H}) .{ }^{13} \mathrm{C}-\mathrm{NMR}\left(91 \mathrm{MHz}, \mathrm{CDCl}_{3}\right) \delta=144.5,140.3$, 132.1, 131.1, 123.0, 94.6, 68.6. FTIR (ATR, neat) $\tilde{v}=3206,3076,2908,1435,1387,1190,1089,1005$, 984, 878, 806. MS (El, $70 \mathrm{eV}$ ) m/z = 311.9 [M] ${ }^{+}, 233.0[\mathrm{M}-\mathrm{Br}]^{+}, 185.0[\mathrm{M}-\mathrm{I}]^{+} . \mathbf{M p} T=107-109$.

The analytical data is in accordance with literature data. ${ }^{[7]}$

\section{(2-Iodo-5-nitrophenyl)methanol (5c) and (2-lodo-3-nitrophenyl)methanol (5c')}

In modification of a reported procedure, ${ }^{[3]}$ nitric acid (7.4 mL, $10.1 \mathrm{~g}, 160 \mathrm{mmol}, 8.00$ eq.) was slowly added to $\mathrm{H}_{2} \mathrm{SO}_{4}$ (22.4 mL, $41.2 \mathrm{~g}, 420 \mathrm{mmol}, 21$ eq.) while maintaining a temperature between -10 and 0 ${ }^{\circ} \mathrm{C}$. At this temperature, $\mathrm{o}$-iodobenzoic acid $(4.96 \mathrm{~g}$,

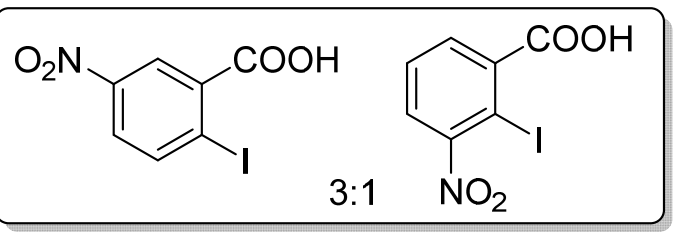
$20.0 \mathrm{mmol}, 1.00$ eq.) was added portionwise. The resulting mixture was stirred for $15 \mathrm{~min}$, allowed to warm to rt and stirred for another $30 \mathrm{~min}$ and then heated to $135^{\circ} \mathrm{C}$ for $1 \mathrm{~h}$. After cooling to $\mathrm{rt}$, the mixture was poured on ice $(50 \mathrm{~g})$ and the resulting solid was collected by filtration. The residue was dissolved in EtOAc $(200 \mathrm{~mL})$, washed with $\mathrm{H}_{2} \mathrm{O}(50 \mathrm{~mL}), \mathrm{Na}_{2} \mathrm{~S}_{2} \mathrm{O}_{3}(50 \mathrm{~mL}, \mathrm{w}=10 \%), \mathrm{H}_{2} \mathrm{O}(50 \mathrm{~mL})$, brine $(50 \mathrm{~mL})$, dried over $\mathrm{Na}_{2} \mathrm{SO}_{4}$ and concentrated under reduced pressure to give a 3:1 mixture of 2-iodo5-nitro- (S1c) and 2-iodo-3-nitro-benzoic acid (S1c', $5.46 \mathrm{~g}, 18.6 \mathrm{mmol}, 93 \%$ ) as a yellow solid which was analysed via ${ }^{1} \mathrm{H}-\mathrm{NMR}$ and used without further purification.

${ }^{1} \mathrm{H}-N M R\left(200 \mathrm{MHz}\right.$, DMSO- $\left.d_{6}\right) \delta=8.40(\mathrm{~d}, J=2.7 \mathrm{~Hz}, 1 \mathrm{H}, \mathrm{A}), 8.29(\mathrm{~d}, J=8.6 \mathrm{~Hz}, 1 \mathrm{H}, \mathrm{A}), 8.00$ (dd, $J=8.6$, $2.8 \mathrm{~Hz}, 1 \mathrm{H}, \mathrm{A}$ ), 7.92 (dd, J = 7.8, 1.7 Hz, 1H, B), 7.78 (dd, $J=7.7,1.7 \mathrm{~Hz}, 1 \mathrm{H}, \mathbf{B}$ ), 7.66 (dd, $J=7.7,7.7 \mathrm{~Hz}$, $1 \mathrm{H}, \mathbf{B}) .(3: 1$ mixture of $\mathbf{A}$ and $\mathbf{B})$. The analytical data is in accordance with literature data. ${ }^{[8]}$ 
Following GP2, the reaction of a 3:1 mixture of 2-iodo-5-nitrobenzoic acid (S1c) and 2-iodo-3nitrobenzoic acid (S1c') (2.49 g, $8.50 \mathrm{mmol})$ with $\mathrm{NaBH}_{4}(933 \mathrm{mg}, 24.7 \mathrm{mmol})$ and $\mathrm{I}_{2}(1.62 \mathrm{~g}, 6.38 \mathrm{mmol})$ gave after separation of the isomers via column chromatography (silica, cyclohexane/ethyl acetate 10:1 $\rightarrow$ 4:1) gave (2-iodo-5-nitrophenyl)methanol (5c, $1.63 \mathrm{~g}, 5.85 \mathrm{mmol}, 67 \%$ ) as a colourless solid and (2-iodo-3-nitrophenyl)methanol (5' $, 475 \mathrm{mg}, 1.70 \mathrm{mmol}, 20 \%$ ) as a yellow solid.

${ }^{1} \mathrm{H}-\mathrm{NMR}\left(200 \mathrm{MHz}, \mathrm{CDCl}_{3}\right) \delta=8.18(\mathrm{~d}, J=2.7 \mathrm{~Hz}, 1 \mathrm{H}), 8.07(\mathrm{~d}, J=8.5 \mathrm{~Hz}$, $1 \mathrm{H}), 7.81(\mathrm{dd}, J=8.5,2.8 \mathrm{~Hz}, 1 \mathrm{H}), 5.85(\mathrm{t}, J=5.4 \mathrm{~Hz}, 1 \mathrm{H}), 4.45(\mathrm{~d}, J=5.1 \mathrm{~Hz}$, 2H). ${ }^{13}$ C-NMR (50 MHz, $\left.\mathrm{CDCl}_{3}\right) \delta=147.8,146.3,139.9,122.7,121.0,105.5$, 66.8. FTIR (ATR, neat) $\tilde{v}=3202,3091,2906,1601,1567,1516,1335,1193$,

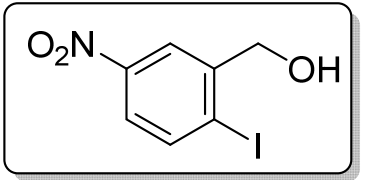
1043, 1014, 907, 806, 736. MS (EI, 70 eV) $\mathrm{m} / \mathrm{z}=279.0[\mathrm{M}]^{+,}, 232.9\left[\mathrm{M}-\mathrm{NO}_{2}\right]^{+}, 152.0[\mathrm{M}-\mathrm{I}]^{+}$ Mp $T=120-122^{\circ} \mathrm{C}$. The analytical data is in accordance with literature data. ${ }^{[9]}$

${ }^{1} \mathrm{H}-\mathrm{NMR}\left(200 \mathrm{MHz}, \mathrm{CDCl}_{3}\right) \delta=7.72-7.64(\mathrm{~m}, 1 \mathrm{H}), 7.55(\mathrm{dd}, J=8.0,2.0 \mathrm{~Hz}, 1 \mathrm{H}), 7.46(\mathrm{t}, J=7.7 \mathrm{~Hz}, 1 \mathrm{H})$, $4.73(\mathrm{~s}, 2 \mathrm{H}), 2.59(\mathrm{~s}, 1 \mathrm{H}) .{ }^{13} \mathrm{C}-\mathrm{NMR}\left(50 \mathrm{MHz}, \mathrm{CDCl}_{3}\right) \delta=154.5,145.9,130.5,129.1$, 123.4, 88.1, 69.7. FTIR (ATR, neat) $\tilde{v}=3277,3075,2926,1586,1523,1418,1345$, 1048, 1018, 789. MS (El, 70 eV) $\mathrm{m} / z=279.0[\mathrm{M}]^{+}, 262.0[\mathrm{M}-\mathrm{OH}]^{+}, 231.9[\mathrm{M}-$ $\left.\mathrm{HNO}_{2}\right]^{+}$. Mp $T=91-92{ }^{\circ} \mathrm{C}$. The analytical data is in accordance with literature data. ${ }^{[9]}$

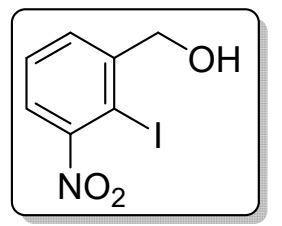

\section{(5-Fluoro-2-iodophenyl)methanol (5d)}

Following GP2, the reaction of 5-fluoro-2-iodobenzoic acid $(665 \mathrm{mg}$, $2.50 \mathrm{mmol})$ with $\mathrm{NaBH}_{4}(274 \mathrm{mg}, 7.25 \mathrm{mmol})$ and $\mathrm{I}_{2}(476 \mathrm{mg}, 1.88 \mathrm{mmol})$ gave after filtration over silica (cyclohexane/ethyl acetate 4:1) (5-fluoro-2iodophenyl)methanol (5d, $577 \mathrm{mg}, 2.19 \mathrm{mmol}, 87 \%$ ) as a colourless solid.

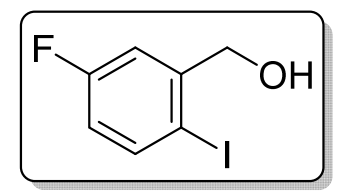

${ }^{1} \mathrm{H}-\mathrm{NMR}\left(601 \mathrm{MHz}, \mathrm{CDCl}_{3}\right) \delta=7.74(\mathrm{dd}, J=8.6,3.1 \mathrm{~Hz}, 1 \mathrm{H}), 7.25(\mathrm{dd}, J=9.3,3.1 \mathrm{~Hz}, 1 \mathrm{H}), 6.76(\mathrm{td}, J=$ 8.3, $3.1 \mathrm{~Hz}, 1 \mathrm{H}), 4.63(\mathrm{~d}, J=6.1 \mathrm{~Hz}, 2 \mathrm{H}), 2.07(\mathrm{t}, J=6.1 \mathrm{~Hz}, 1 \mathrm{H}) .{ }^{13} \mathrm{C}-\mathrm{NMR}\left(151 \mathrm{MHz}, \mathrm{CDCl}_{3}\right) \delta=163.1(\mathrm{~d}$, $J=247.8 \mathrm{~Hz}$ ), $145.0(\mathrm{~d}, J=6.9 \mathrm{~Hz}), 140.1(\mathrm{~d}, J=7.6 \mathrm{~Hz}), 116.4(\mathrm{~d}, J=22.0 \mathrm{~Hz}), 115.5(\mathrm{~d}, J=23.4 \mathrm{~Hz}), 89.1$ $(\mathrm{d}, J=2.7 \mathrm{~Hz}), 68.8 .{ }^{19} \mathrm{~F}-\mathrm{NMR}\left(565 \mathrm{MHz}, \mathrm{CDCl}_{3}\right) \delta=-113.4$. FTIR (ATR, neat) $\tilde{v}=3286,1578,1458,1437$, $1356,1263,1149,1058,1013,808$. HRMS (EI, $70 \mathrm{eV}) \mathrm{m} / \mathrm{z} 251.94426$. Calculated for $\mathrm{C}_{7} \mathrm{H}_{6} \mathrm{FIO}^{+\cdot}: \mathrm{m} / \mathrm{z}$ 251.94419. $\mathrm{Mp} T=111.5-113$. The analytical data is in accordance with literature data. ${ }^{[10]}$

\section{(2-lodo-5-(trifluoromethyl)phenyl)methanol (5e)}

Following GP2, the reaction of 2-iodo-(5-trifluoromethyl)benzoic acid $(790 \mathrm{mg}, 2.50 \mathrm{mmol})$ with $\mathrm{NaBH}_{4}(274 \mathrm{mg}, 7.25 \mathrm{mmol})$ and $\mathrm{I}_{2}(476 \mathrm{mg}$, $1.88 \mathrm{mmol}$ ) gave after filtration over silica (cyclohexane/ethyl acetate 4:1) (2-iodo-5-(trifluoromethyl)phenyl)methanol (5e, $682 \mathrm{mg}, 2.25 \mathrm{mmol}, 90 \%$ )

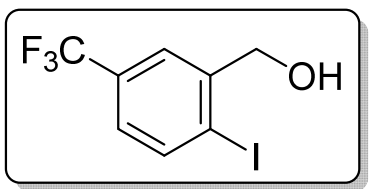
as a colourless solid.

${ }^{1} \mathrm{H}-\mathrm{NMR}\left(601 \mathrm{MHz}, \mathrm{CDCl}_{3}\right) \delta=7.94(\mathrm{~d}, J=8.2 \mathrm{~Hz}, 1 \mathrm{H}), 7.76-7.75(\mathrm{~m}, 1 \mathrm{H}), 7.31-7.21(\mathrm{~m}, 1 \mathrm{H}), 4.72(\mathrm{~d}$, $J=6.0 \mathrm{~Hz}, 2 \mathrm{H}), 2.07(\mathrm{t}, J=6.1 \mathrm{~Hz}, 1 \mathrm{H}) .{ }^{13} \mathrm{C}-\mathrm{NMR}\left(151 \mathrm{MHz}, \mathrm{CDCl}_{3}\right) \delta=143.7,139.6,131.0$ (q, $J=32.7$ $\mathrm{Hz}), 125.5(\mathrm{q}, J=3.5 \mathrm{~Hz}), 124.5(\mathrm{q}, J=3.5 \mathrm{~Hz}), 122.1$ (q, $J=272.8 \mathrm{~Hz}), 100.8,68.8 .{ }^{19} \mathrm{~F}-\mathrm{NMR}(565 \mathrm{MHz}$, $\mathrm{CDCl}_{3}$ ) $\delta=-62.9$. FTIR (ATR, neat) $\tilde{v}=3289,1600,1465,1324,1256,1175,1118,1044,1009,897,829$, 743. HRMS (El, 70 eV) $\mathrm{m} / z=301.94107$. Calculated for $\mathrm{C}_{8} \mathrm{H}_{6} \mathrm{~F}_{3} \mathrm{O}^{+}: \mathrm{m} / \mathrm{z}$ 301.94100. $\mathrm{Mp} T=80.5-81.5$. The analytical data is in accordance with literature data. ${ }^{[11]}$ 


\section{(5-Chloro-2-iodophenyl)methanol (5f)}

Following GP1a, the reaction of 2-amino-5-chlorobenzoic acid (1.72 g) gave 5-chloro-2-iodobenzoic acid (S1f, $2.22 \mathrm{~g}, 7.87 \mathrm{mmol}, 79 \%$ ) as a colourless solid which still contained some impurities $(\approx 5 \%)$ but was used without further purification.

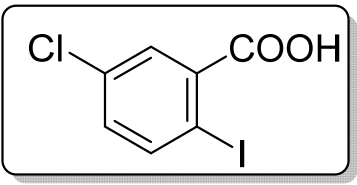

${ }^{1} \mathrm{H}-\mathrm{NMR}\left(600 \mathrm{MHz}\right.$, DMSO- $\left.d_{6}\right) \delta=13.61(\mathrm{brs}, 1 \mathrm{H}), 7.97(\mathrm{~d}, J=8.4 \mathrm{~Hz}, 1 \mathrm{H}), 7.72(\mathrm{~d}, J=2.6 \mathrm{~Hz}, 1 \mathrm{H}), 7.32$ $(\mathrm{dd}, J=8.4,2.6 \mathrm{~Hz}, 1 \mathrm{H}) .{ }^{13} \mathrm{C}-\mathrm{NMR}\left(151 \mathrm{MHz}\right.$, DMSO- $\left.d_{6}\right) \delta=167.0,142.1,138.9,133.2,132.2,129.5$, 92.1. FTIR (ATR, neat) $\tilde{v}=1694,1673,1574,1400,1290,1246,1117,1017,780$, 749. HRMS (ESI-, MeOH) $m / z=280.88690[\mathrm{M}-\mathrm{H}]^{-}$. Calculated for $\mathrm{C}_{7} \mathrm{H}_{3} \mathrm{ClIO}_{2}^{-}: \mathrm{m} / z=280.88717$. $\mathrm{Mp} T=154-157$. The analytical data is in accordance with literature data. ${ }^{[12]}$

Following GP2, the reaction of 5-chloro-2-iodobenzoic acid (S1f, $1.41 \mathrm{~g}$, $5.00 \mathrm{mmol})$ with $\mathrm{NaBH}_{4}(549 \mathrm{mg}, 14.5 \mathrm{mmol})$ and $\mathrm{I}_{2}(952 \mathrm{mg}, 3.75 \mathrm{mmol})$ gave after column chromatography (silica, cyclohexane/ethyl acetate 10:1) (5chloro-2-iodophenyl)methanol (5f, $1.08 \mathrm{~g}, 4.01 \mathrm{mmol}, 80 \%$ ) as a colourless

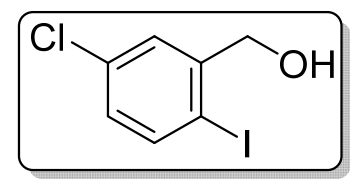
solid.

${ }^{1} \mathrm{H}-\mathrm{NMR}\left(601 \mathrm{MHz}, \mathrm{CDCl}_{3}\right) \delta=7.71(\mathrm{~d}, J=8.3 \mathrm{~Hz}, 1 \mathrm{H}), 7.48(\mathrm{~d}, J=2.5 \mathrm{~Hz}, 1 \mathrm{H}), 6.99$ (dd, $J=8.3,2.6 \mathrm{~Hz}$, 1H), $4.63(\mathrm{~s}, 2 \mathrm{H}), 2.10(\mathrm{~s}, 1 \mathrm{H}) .{ }^{13} \mathrm{C}-\mathrm{NMR}\left(151 \mathrm{MHz}, \mathrm{CDCl}_{3}\right) \delta=144.3,140.0,135.0,129.2,128.2,93.6$, 68.7. FTIR (ATR, neat) $\tilde{v}=3289,3212,1452,1436,1391,1193,1101,1055,1007,974,808$. MS (EI, 70 eV) $\mathrm{m} / z=268.0[\mathrm{M}]^{+}, 233.9[\mathrm{M}-\mathrm{Cl}]^{+}, 141.0[\mathrm{M}-\mathrm{I}]^{+} \mathrm{Mp} T=118-119.5$. The analytical data is in accordance with literature data. ${ }^{[7]}$

\section{3-(Hydroxymethyl)-4-iodobenzonitrile (5g)}

In modification of a reported procedure, ${ }^{[3]}$ 2-Amino-5-bromobenzoic acid ( $5.40 \mathrm{~g}, 25.0 \mathrm{mmol}, 1$ eq.) was dissolved in $\mathrm{MeOH}(100 \mathrm{~mL})$. After addition of $\mathrm{H}_{2} \mathrm{SO}_{4}(6.00 \mathrm{~mL}, 5.00 \mathrm{mmol}, 0.2$ eq., $98 \% \mathrm{v} / \mathrm{v})$ the mixture was heated under reflux for $16 \mathrm{~h}$. The mixture was concentrated under reduced pressure and sat. $\mathrm{Na}_{2} \mathrm{CO}_{3}(50 \mathrm{~mL})$ and EtOAc $(50 \mathrm{~mL})$ were added. The

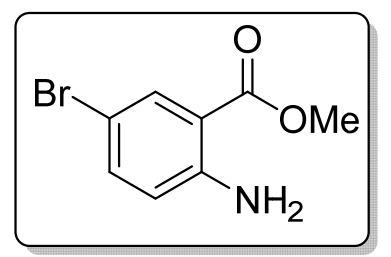
phases were separated, the aqueous phase was extracted with EtOAc $(3 \times 50 \mathrm{~mL})$ and the combined organic phases were dried over $\mathrm{Na}_{2} \mathrm{SO}_{4}$ and concentrated under reduced pressure. After purification of the residue by column chromatography (silica, cyclohexane/ethyl acetate 7:1 $\rightarrow$ 4:1) methyl 2amino-5-bromobenzoate ( $3.80 \mathrm{~g}, 16.7 \mathrm{mmol}, 66 \%$ ) was obtained as a colourless solid.

${ }^{1} \mathrm{H}-\mathrm{NMR}\left(600 \mathrm{MHz}, \mathrm{CDCl}_{3}\right) \delta=7.96(\mathrm{~d}, J=2.4 \mathrm{~Hz}, 1 \mathrm{H}), 7.31$ (dd, $\left.J=8.8,2.4 \mathrm{~Hz}, 1 \mathrm{H}\right), 6.55(\mathrm{~d}, J=8.8 \mathrm{~Hz}$, 1H), 5.75 (s, 2H), $3.86(\mathrm{~s}, 3 \mathrm{H}) .{ }^{13} \mathrm{C}-\mathrm{NMR}\left(151 \mathrm{MHz}, \mathrm{CDCl}_{3}\right) \delta=167.4,149.3,136.7,133.4,118.3,112.0$, 107.3, 51.7. FTIR (ATR, neat) $\tilde{v}=3454,3355,1948,1683,1625,1558,1442,1296,1236,1082,967$, 824, 786. MS (El, 70 eV) $\mathrm{m} / \mathrm{z}=229.0[\mathrm{M}]^{+}, 197.0[\mathrm{M}-\mathrm{MeOH}]^{+}, 170.0[\mathrm{M}-\mathrm{COOMe}]^{+} \mathbf{M p ~} T=72-73$.

The analytical data is in accordance with literature data. ${ }^{[13]}$

In a pressure vial methyl 2-amino-5-bromobenzoate $(1.15 \mathrm{~g}, 5.00 \mathrm{mmol}$, 1.00 eq.) and CuCN (493 mg, $5.50 \mathrm{mmol}, 1.10$ eq.) were suspended in dry DMF $(20 \mathrm{~mL})$ and stirred for $4 \mathrm{~h}$ at $160{ }^{\circ} \mathrm{C}$. Et ${ }_{2} \mathrm{O}(50 \mathrm{~mL})$ and $2 \mathrm{~N} \mathrm{Na}_{2} \mathrm{CO}_{3}$ $(50 \mathrm{~mL})$ were added and the phases were separated. The aqueous phase was extracted with $\mathrm{Et}_{2} \mathrm{O}(2 \times 50 \mathrm{~mL})$ and the combined organic phases were washed with $\mathrm{H}_{2} \mathrm{O}(6 \times 10 \mathrm{~mL})$, dried over $\mathrm{Na}_{2} \mathrm{SO}_{4}$ and concentrated under

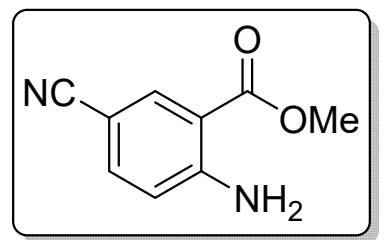
reduced pressure. The residue was purified via column chromatography (silica, cyclohexane/ethyl acetate $5: 1 \rightarrow 3: 1$ ) to obtain methyl 2-amino-5-cyanobenzoate ( $484 \mathrm{mg}, 2.75 \mathrm{mmol}, 55 \%$ ) as a slightly yellow solid. 
${ }^{1} \mathrm{H}-\mathrm{NMR}\left(600 \mathrm{MHz}, \mathrm{CDCl}_{3}\right) \delta=8.19(\mathrm{~d}, J=2.0 \mathrm{~Hz}, 1 \mathrm{H}), 7.45(\mathrm{dd}, J=8.7,2.0 \mathrm{~Hz}, 1 \mathrm{H}), 6.67(\mathrm{~d}, J=8.7 \mathrm{~Hz}$, 1H), 6.29 (s, 2H), $3.90(\mathrm{~s}, 3 \mathrm{H}) .{ }^{13} \mathrm{C}-\mathrm{NMR}\left(151 \mathrm{MHz}, \mathrm{CDCl}_{3}\right) \delta=167.1,153.0,136.9,136.3,119.3,117.0$, 110.5, 98.7, 52.1. FTIR (ATR, neat) $\tilde{v}=3465,3359,2212,1708,1629,1591,1550,1439,1263,1188$, 1077, 821. MS (EI, 70 eV) $\mathrm{m} / \mathrm{z}=176.1$ [M] $^{+}, 144.1$ [M-MeOH] $^{+}, 117.0[\mathrm{M}-\mathrm{COOMe}]^{+} \mathrm{Mp} T=129-131$. The analytical data is in accordance with literature data. ${ }^{[14]}$

Following GP1a, methyl 2-amino-5-cyanobenzoate $(317 \mathrm{mg}, 1.80 \mathrm{mmol}$, 1.00 eq.) and $\mathrm{TsOH} \cdot \mathrm{H}_{2} \mathrm{O}(1.03 \mathrm{~g}, 5.40 \mathrm{mmol}, 3.00$ eq.) were suspended in Acetonitrile $(8 \mathrm{~mL})$. The mixture was cooled to $10-15^{\circ} \mathrm{C}$. A solution of $\mathrm{NaNO}_{2}(278 \mathrm{mg}, 3.60 \mathrm{mmol}, 2$ eq.) and $\mathrm{KI}(747 \mathrm{mg}, 4.50 \mathrm{mmol}, 2.50$ eq.) in $\mathrm{H}_{2} \mathrm{O}(1 \mathrm{~mL})$ was added dropwise over $1 \mathrm{~h}$. After complete addition, the

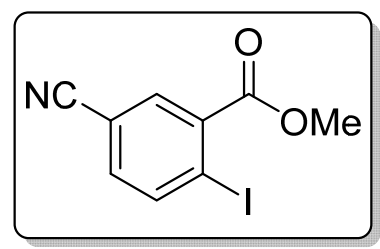
resulting dark coloured mixture was allowed to stir for another $1 \mathrm{~h}$ and then $1 \mathrm{~N} \mathrm{HCl}(10 \mathrm{~mL})$ and EtOAc $(20 \mathrm{~mL})$ were added. The phases were separated and the aqueous phases was extracted with EtOAc ( 2 $x 20 \mathrm{~mL})$. The combined organic phases were washed with water $(50 \mathrm{~mL}), \mathrm{Na}_{2} \mathrm{~S}_{2} \mathrm{O}_{3}(10 \% \mathrm{w} / \mathrm{w}, 50 \mathrm{~mL})$ and brine $(20 \mathrm{~mL})$, dried over $\mathrm{Na}_{2} \mathrm{SO}_{4}$ and concentrated under reduced pressure. The residue was purified by filtration over silica (cyclohexane/ethyl acetate 4:1) to obtain methyl 5-cyano-2iodobenzoate ( $401 \mathrm{mg}, 1.39 \mathrm{mmol}, 78 \%$ ) as a colourless solid.

${ }^{1} \mathrm{H}-\mathrm{NMR}\left(600 \mathrm{MHz}, \mathrm{CDCl}_{3}\right) \delta=8.15(\mathrm{~d}, J=8.2 \mathrm{~Hz}, 1 \mathrm{H}), 8.07(\mathrm{~d}, J=2.0 \mathrm{~Hz}, 1 \mathrm{H}), 7.39$ (dd, $J=8.2,2.0 \mathrm{~Hz}$, 1H), 3.97 (s, 3H). ${ }^{13} \mathrm{C}-\mathrm{NMR}\left(151 \mathrm{MHz}, \mathrm{CDCl}_{3}\right) \delta=165.1,142.6,136.2,134.7,134.0,117.3,112.5,100.4$, 53.1. FTIR (ATR, neat) $\tilde{v}=3057,2233,1729,1584,1431,1298,1245,1195,1104,1020,973,828$. HRMS (El, $70 \mathrm{eV}$ ) $\mathrm{m} / \mathrm{z}=286.94331$ Calculated for $\mathrm{C}_{9} \mathrm{H}_{6} \mathrm{INO}_{2}{ }^{+}: \mathrm{m} / \mathrm{z}=286.94378 \mathrm{Mp} T=119-121$.

Methyl 5-cyano-2-iodobenzoate ( $345 \mathrm{mg}, 1.20 \mathrm{mmol}, 1.00$ eq.) was dissolved in $\mathrm{MeOH} / \mathrm{THF}(2: 1 \mathrm{v} / \mathrm{v}, 10 \mathrm{~mL})$ and at $0{ }^{\circ} \mathrm{C} \mathrm{NaBH}_{4}(454 \mathrm{mg}, 12.0 \mathrm{mmol}, 10$ eq.) was added portionwise over $2 \mathrm{~h}$ and the suspenseion was stirred for $1 \mathrm{~h}$. $\mathrm{Et}_{2} \mathrm{O}$ $(50 \mathrm{~mL}), \mathrm{H}_{2} \mathrm{O}(10 \mathrm{~mL})$ and $1 \mathrm{~N} \mathrm{HCl}(10 \mathrm{~mL})$ were added, the phases were separated and the aqueous phase was extracted with $\mathrm{Et}_{2} \mathrm{O}(2 \times 50 \mathrm{~mL})$. The

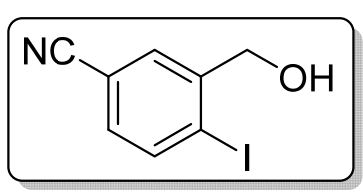
combined organic phases were dried over $\mathrm{Na}_{2} \mathrm{SO}_{4}$, concentrated under reduced pressure and purified via column chromatography (cyclohexane/ethyl acetate 10:1) to obtain 3-(hydroxymethyl)-4iodobenzonitrile $(5 \mathrm{~g}, 127 \mathrm{mg}, 0.490 \mathrm{mmol}, 41 \%)$

${ }^{1} \mathrm{H}-\mathrm{NMR}\left(600 \mathrm{MHz}, \mathrm{DMSO}-d_{6}\right) \delta=8.05(\mathrm{~d}, J=8.0 \mathrm{~Hz}, 1 \mathrm{H}), 7.75(\mathrm{~s}, 1 \mathrm{H}), 7.48(\mathrm{dd}, J=8.0,1.6 \mathrm{~Hz}, 1 \mathrm{H}), 5.70$ $(\mathrm{s}, 1 \mathrm{H}), 4.42(\mathrm{~s}, 2 \mathrm{H}) .{ }^{13} \mathrm{C}-\mathrm{NMR}\left(151 \mathrm{MHz}\right.$, DMSO-d $\left.\boldsymbol{d}_{6}\right) \delta=145.8,139.8,131.6,129.9,118.6,111.0,103.6$, 66.8. FTIR (ATR, neat) $\tilde{v}=3460,2234,1583,1427,1394,1299,1221,1055,1010,891,821$. MS (EI, 70 eV) $m / z=259.0[\mathrm{M}]^{+}$. Mp $T=147-148.5$.

\section{(4-Bromo-2-iodophenyl)methanol (5h)}

Following GP1a, the reaction of 2-amino-4-bromobenzoic acid ( $2.16 \mathrm{~g})$ gave 4bromo-2-iodobenzoic acid (S1h, $2.21 \mathrm{~g}, 7.06 \mathrm{mmol}, 71 \%$ ) as a colourless solid.

${ }^{1} \mathrm{H}-\mathrm{NMR}\left(600 \mathrm{MHz}\right.$, DMSO- $\left.d_{6}\right) \delta=13.47(\mathrm{~s}, 1 \mathrm{H}), 8.19(\mathrm{~d}, J=1.8 \mathrm{~Hz}, 1 \mathrm{H}), 7.70$ (dd, $J=8.3,1.8 \mathrm{~Hz}, 1 \mathrm{H}), 7.65$ (d, $J=8.3 \mathrm{~Hz}, 1 \mathrm{H}) .{ }^{13} \mathrm{C}-\mathrm{NMR}$ (151 MHz, DMSO) $\delta$

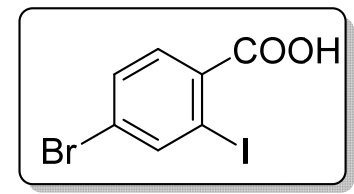
$=167.4,142.1,136.0,131.5,131.2,125.0,95.7$. FTIR (ATR, neat) $\tilde{v}=3064,1672,1422,1291,1542$, 1291, 1249, 1023, 838, 771. HRMS (ESI-, MeOH) $\mathrm{m} / \mathrm{z}=324.83636$ Calculated for $\mathrm{C}_{7} \mathrm{H}_{3} \mathrm{BrIO}_{2}^{-} \mathrm{m} / \mathrm{z}=$ 324.83666. Mp $T=175-177$. 
Following GP2, the reaction of 4-bromo-2-iodobenzoic acid (S1h, $1.63 \mathrm{~g}$, $5.00 \mathrm{mmol}$ ) with $\mathrm{NaBH}_{4}(549 \mathrm{mg}, 14.5 \mathrm{mmol})$ and $\mathrm{I}_{2}(952 \mathrm{mg}, 3.75 \mathrm{mmol})$ gave after filtration over silica (cyclohexane/ethyl acetate 4:1) (4-bromo-2iodophenyl)methanol (5h, $1.39 \mathrm{~g}, 4.44 \mathrm{mmol}, 87 \%)$ as a colourless solid.

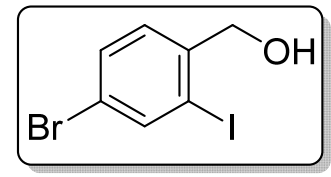

${ }^{1} \mathrm{H}-\mathrm{NMR}\left(600 \mathrm{MHz}, \mathrm{CDCl}_{3}\right) \delta=7.96(\mathrm{~d}, J=1.9 \mathrm{~Hz}, 1 \mathrm{H}), 7.50(\mathrm{dd}, J=8.2,1.9 \mathrm{~Hz}, 1 \mathrm{H}), 7.33(\mathrm{~d}, J=8.2 \mathrm{~Hz}$, $1 \mathrm{H}), 4.62(\mathrm{~d}, J=5.6 \mathrm{~Hz}, 2 \mathrm{H}), 2.06(\mathrm{t}, J=6.0 \mathrm{~Hz}, 1 \mathrm{H}) .{ }^{13} \mathrm{C}-\mathrm{NMR}\left(151 \mathrm{MHz}, \mathrm{CDCl}_{3}\right) \delta=141.6,140.9,131.5$, 129.3, 121.7, 97.3, 68.6. FTIR (ATR, neat) $\tilde{v}=3315,3226,1572,1550,1467,1377,1056,1008,801$, 705. HRMS (EI) $\mathrm{m} / z=311.86442$ Calculated for $\mathrm{C}_{7} \mathrm{H}_{6} \mathrm{BrlO}^{+\cdot} \mathrm{m} / z=311.86413 \mathrm{Mp} T=104-105$.

\section{(3-Bromo-2-iodophenyl)methanol (5i)}

Following GP1a, the reaction of 2-amino-3-bromobenzoic acid ( $2.16 \mathrm{~g})$ gave 3bromo-2-iodobenzoic acid (S1i, $1.25 \mathrm{~g}, 3.83 \mathrm{mmol}, 38 \%$ ) as a colourless solid which still contained some impurities $(\approx 5 \%)$ but was used without further purification.

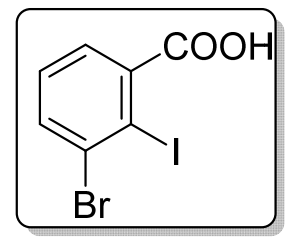

${ }^{1} \mathrm{H}-\mathrm{NMR}\left(601 \mathrm{MHz}\right.$, DMSO- $d_{6}$ ) $\delta=13.54$ (brs, $\left.1 \mathrm{H}\right), 7.82$ (dd, $\left.J=8.0,1.5 \mathrm{~Hz}, 1 \mathrm{H}\right), 7.46$ (dd, $J=7.6,1.5 \mathrm{~Hz}$, $1 \mathrm{H}), 7.38(\mathrm{t}, J=7.8 \mathrm{~Hz}, 1 \mathrm{H}) .{ }^{13} \mathrm{C}-\mathrm{NMR}\left(151 \mathrm{MHz}\right.$, DMSO- $\left.d_{6}\right) \delta=169.0,142.9,133.9,131.3,129.9,127.0$, 100.8. FTIR (ATR, neat) $\tilde{v}=2864,1675,1567,1407,1390,1281,1249,1199,967,750$. HRMS (ESI-, MeOH) $m / z=324.83636$. Calculated for $\mathrm{C}_{7} \mathrm{H}_{3} \mathrm{BrlO}_{2}: \mathrm{m} / \mathrm{z}=324.83666$. The analytical data is in accordance with literature data. ${ }^{[15]}$

Following GP2, the reaction of 3-bromo-2-iodobenzoic acid (S1i, $817 \mathrm{mg}$, $2.50 \mathrm{mmol}$ ) with $\mathrm{NaBH}_{4}(274 \mathrm{mg}, 7.25 \mathrm{mmol})$ and $\mathrm{I}_{2}(476 \mathrm{mg}, 1.88 \mathrm{mmol})$ gave after filtration over silica (cyclohexane/ethyl acetate 4:1) (3-bromo-2iodophenyl)methanol (5i, $611 \mathrm{mg}, 1.95 \mathrm{mmol}, 78 \%$ ) as a colourless solid.

${ }^{1} \mathrm{H}-\mathrm{NMR}\left(600 \mathrm{MHz}, \mathrm{CDCl}_{3}\right) \delta=7.61(\mathrm{~d}, J=7.9 \mathrm{~Hz}, 1 \mathrm{H}), 7.41(\mathrm{~d}, J=7.5 \mathrm{~Hz}, 1 \mathrm{H}), 7.28$

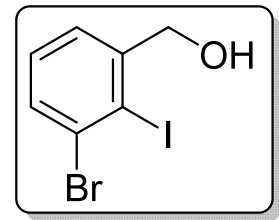
(d, $J=8.0 \mathrm{~Hz}, 1 \mathrm{H}), 4.73(\mathrm{~d}, J=6.3 \mathrm{~Hz}, 2 \mathrm{H}), 2.19$ (t, $J=6.3 \mathrm{~Hz}, 1 \mathrm{H}) .{ }^{13} \mathrm{C}-\mathrm{NMR}\left(151 \mathrm{MHz}, \mathrm{CDCl}_{3}\right) \delta=146.2$, 131.7, 131.0, 129.5, 126.2, 104.1, 71.0. FTIR (ATR, neat) $\tilde{v}=3295,1428,1397,1345,1215,1172,1133$, 1048, 980, 769. HRMS (El, 70 eV) $\mathrm{m} / z=311.86442$ Calculated for $\mathrm{C}_{7} \mathrm{H}_{6} \mathrm{BrlO}^{+}: \mathrm{m} / \mathrm{z}=311.86413 \mathrm{Mp} T=$ $110-112$.

\section{(3,5-Dichloro-2-iodophenyl)methanol (5j)}

Following GP1a, the reaction of 2-amino-3,5-dichlorobenzoic acid (2.06 g) gave 3,5-dichloro-2-iodobenzoic acid (S1j, $1.39 \mathrm{~g}, 4.39 \mathrm{mmol}, 44 \%$ ) as a colourless solid which still contained some impurities $(\approx 5 \%)$ but was used without further purification.

${ }^{1} \mathrm{H}-\mathrm{NMR}\left(600 \mathrm{MHz}\right.$, DMSO- $\left.d_{6}\right) \delta=13.85(\mathrm{~s}, 1 \mathrm{H}), 7.87(\mathrm{~d}, J=2.4 \mathrm{~Hz}, 1 \mathrm{H}), 7.57$

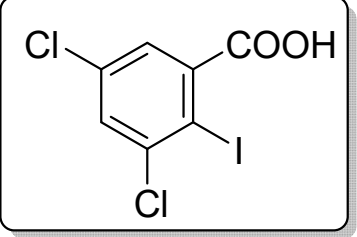
(d, $J=2.4 \mathrm{~Hz}, 1 \mathrm{H}) .{ }^{13} \mathrm{C}-\mathrm{NMR}\left(151 \mathrm{MHz}\right.$, DMSO- $\left.d_{6}\right) \delta=167.7,143.8,140.3,134.0,129.8,126.6,96.8$. FTIR (ATR, neat) $\tilde{v}=3070,1693,1547,1418,1367,1281,1242,1156,1019,870,819$. HRMS (ESI-, MeOH) $m / z=314.84790$. Calculated for $\mathrm{C}_{7} \mathrm{H}_{2} \mathrm{Cl}_{2} \mathrm{IO}_{2}^{-} \mathrm{m} / z=314,84820$. Mp $T=177-179$.

Following GP2, the reaction of 3,5-dichloro-2-iodobenzoic acid (S1j, $792 \mathrm{mg}$, $2.50 \mathrm{mmol}$ ) with $\mathrm{NaBH}_{4}(274 \mathrm{mg}, 7.25 \mathrm{mmol})$ and $\mathrm{I}_{2}(476 \mathrm{mg}, 1.88 \mathrm{mmol})$ gave after filtration over silica (cyclohexane/ethyl acetate 4:1) (3,5-dichloro-2iodophenyl)methanol (5j, $744 \mathrm{mg}, 2.46 \mathrm{mmol}, 98 \%$ ) as a colourless solid.

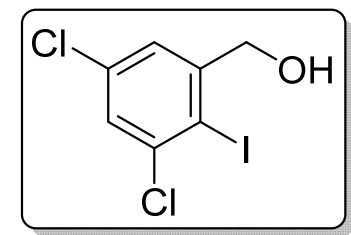


${ }^{1} \mathrm{H}-\mathrm{NMR}\left(601 \mathrm{MHz}, \mathrm{CDCl}_{3}\right) \delta=7.44-7.41(\mathrm{~m}, 1 \mathrm{H}), 7.41-7.38(\mathrm{~m}, 1 \mathrm{H}), 4.66(\mathrm{~d}, J=6.1 \mathrm{~Hz}, 2 \mathrm{H}), 2.15(\mathrm{t}$, $J=6.1 \mathrm{~Hz}, 1 \mathrm{H}) .{ }^{13} \mathrm{C}-\mathrm{NMR}\left(151 \mathrm{MHz}, \mathrm{CDCl}_{3}\right) \delta=146.9,139.8,135.3,127.8,125.9,98.0,69.8$. FTIR (ATR, neat) $\tilde{v}=3183,2889,1551,1418,1399,1384,1216,1171,1094,1058,1008,858$. HRMS (EI, $70 \mathrm{eV}$ ) $\mathrm{m} / \mathrm{z}=301.87562$. Calculated for $\mathrm{C}_{7} \mathrm{H}_{5} \mathrm{Cl}_{2} \mathrm{IO}^{+\cdot} \mathrm{m} / \mathrm{z}=301.87567 . \mathrm{Mp} T=95.5-97$.

\subsubsection{Derivatives of Primary Benzyl Alcohols}

\section{2-lodobenzyl acetate (10)}

o-lodobenzylalcohol (5a, $585 \mathrm{mg}, 2.50 \mathrm{mmol}, 1.00$ eq.) was dissolved in dry DCM (12.5 mL), cooled to $0{ }^{\circ} \mathrm{C}$ and triethylamine $\left(520 \mu \mathrm{L}, 3.75 \mathrm{mmol}, 1.5\right.$ eq.) and $\mathrm{Ac}_{2} \mathrm{O}$ ( $295 \mu \mathrm{L}, 3.13 \mathrm{mmol}, 1.25$ eq.) were added. The solution was warmed up to $\mathrm{rt}$ overnight. Water $(10 \mathrm{~mL})$ was added. The phases were separated and the aqueous

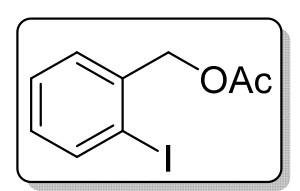
phase was extracted with DCM $(2 \times 15 \mathrm{~mL})$. The combined organic phases were washed with brine $(15 \mathrm{~mL})$, dried over $\mathrm{Na}_{2} \mathrm{SO}_{4}$ and concentrated under reduced pressure. Column chromatography of the crude product (silica, cyclohexane/ethyl acetate 5:1) afforded the product (10,636 mg, $2.30 \mathrm{mmol}$, $92 \%)$ as a colourless liquid.

${ }^{1} \mathrm{H}-\mathrm{NMR}\left(600 \mathrm{MHz}, \mathrm{CDCl}_{3}\right) \delta=7.86(\mathrm{~d}, J=7.9 \mathrm{~Hz}, 1 \mathrm{H}), 7.38(\mathrm{dd}, J=7.6,1.9 \mathrm{~Hz}, 1 \mathrm{H}), 7.35(\mathrm{td}, J=7.4,0.9$ $\mathrm{Hz}, 1 \mathrm{H}), 7.03$ (td, $J=7.6,1.9 \mathrm{~Hz}, 1 \mathrm{H}), 5.13(\mathrm{~s}, 2 \mathrm{H}), 2.15$ (s, 3H). ${ }^{13} \mathrm{C}-\mathrm{NMR}\left(151 \mathrm{MHz}, \mathrm{CDCl}_{3}\right) \delta=170.6$, 139.5, 138.3, 129.8, 129.5, 128.3, 98.4, 70.1, 20.9. FTIR (ATR, neat) $\tilde{v}=3056,2953,1732,1566,1437$, $1378,1360,1218,1012,746 . \mathbf{M S}(\mathbf{E l}, 70 \mathrm{eV}) \mathrm{m} / \mathrm{z}=276.0[\mathrm{M}]^{+}, 149.1[\mathrm{M}-\mathrm{I}]^{+}$. The analytical data is in accordance with literature data. ${ }^{[16]}$

\section{1-lodo-2-(methoxymethyl)benzene (11)}

In modification of a reported procedure, ${ }^{[17]} O$-iodobenzylalcohol $(5 \mathrm{a}, 585 \mathrm{mg}$, $2.50 \mathrm{mmol}, 1.00$ eq.) was dissolved in dry DMF $(12.5 \mathrm{~mL})$. After cooling to $0{ }^{\circ} \mathrm{C}$, $\mathrm{NaH}(200 \mathrm{mg}, 5.00 \mathrm{mmol}, 60 \% \mathrm{w} / \mathrm{w}$ in mineral oil, 2.00 eq.) was added and the

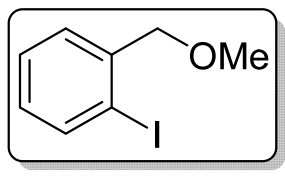
resulting suspension was stirred for $10 \mathrm{~min}$. lodomethane $(311 \mu \mathrm{L}, 710 \mathrm{mg}, 5.00 \mathrm{mmol}, 2.00 \mathrm{eq}$.) was added and the cooling bath was removed. After stirring for $1 \mathrm{~h}$ at $\mathrm{rt}$, triethylamine $(1 \mathrm{~mL})$ was added and the suspension was stirred for another $30 \mathrm{~min}$. Water $(15 \mathrm{~mL})$ was added, the phases were separated and the aqueous phase was extracted with DCM $(2 \times 15 \mathrm{~mL})$. The combined organic phases were washed with water $(30 \mathrm{~mL})$, dried over $\mathrm{Na}_{2} \mathrm{SO}_{4}$ and concentrated under reduced pressure. The residue was purified by filtration over silica (cyclohexane/ethyl acetate 10:1) to obtain the product (11, $615 \mathrm{mg}, 2.48 \mathrm{mmol}, 99 \%$ ) as a colourless liquid that solidifies at $4{ }^{\circ} \mathrm{C}$.

${ }^{1} \mathrm{H}-\mathrm{NMR}\left(601 \mathrm{MHz}, \mathrm{CDCl}_{3}\right) \delta=7.83(\mathrm{dd}, J=7.9,0.9 \mathrm{~Hz}, 1 \mathrm{H}), 7.43-7.39(\mathrm{~m}, 1 \mathrm{H}), 7.35(\mathrm{td}, J=7.6,1.0 \mathrm{~Hz}$, 1H), $7.01-6.96(\mathrm{~m}, 1 \mathrm{H}), 4.45(\mathrm{~s}, 2 \mathrm{H}), 3.47$ (s, 3H). ${ }^{13} \mathrm{C}-\mathrm{NMR}\left(151 \mathrm{MHz}, \mathrm{CDCl}_{3}\right) \delta=140.4,139.2,129.1$, 128.6, 128.2, 97.7, 78.3, 58.6. FTIR (ATR, neat) $\tilde{v}=2921,1565,1437,1378,1193,1116,1099,1013$, 973, 744. MS (El, $70 \mathrm{eV}) \mathrm{m} / \mathrm{z}=248.0[\mathrm{M}]^{+}, 217.0[\mathrm{M}-\mathrm{OMe}]^{+}, 121.1[\mathrm{M}-\mathrm{I}]^{+}$. The analytical data is in accordance with literature data. ${ }^{[17]}$ 


\section{Ethyl (2-iodobenzyl) carbonate (12)}

o-lodobenzylalcohol $(5 \mathrm{a}, 585 \mathrm{mg}, 2.50 \mathrm{mmol})$ was dissolved in dry DCM $(12.5 \mathrm{~mL})$ and at $0{ }^{\circ} \mathrm{C}$, dry pyridine $(303 \mu \mathrm{L}, 297 \mathrm{mg}, 3.75 \mathrm{mmol}, 1.5$ eq.) and ClCOOEt ( $298 \mu \mathrm{L}, 339 \mathrm{mg}, 3.13 \mathrm{mmol}, 1.25$ eq.) were added. The cooling bath was removed and the solution was allowed to stir at rt over night. After completion of the reaction, sat. $\mathrm{NaHCO}_{3}(15 \mathrm{~mL})$ was added. The phases

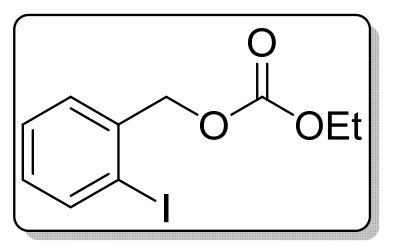
were separated and the aqueous phase was extracted with DCM $(2 \times 15 \mathrm{~mL})$. The combined organic phases were washed with water $(30 \mathrm{~mL})$, dried over $\mathrm{Na}_{2} \mathrm{SO}_{4}$, concentrated under reduced pressure and the residue was purified by filtration over silica (cyclohexane) to obtain ethyl (2-iodobenzyl) carbonate (12, $665 \mathrm{mg}, 2.17 \mathrm{mmol}, 87 \%)$ as a colourless solid.

${ }^{1} \mathrm{H}-\mathrm{NMR}\left(600 \mathrm{MHz}, \mathrm{CDCl}_{3}\right) \delta=7.85(\mathrm{dd}, J=7.9,1.0 \mathrm{~Hz}, 1 \mathrm{H}), 7.42(\mathrm{dd}, J=7.6,1.5 \mathrm{~Hz}, 1 \mathrm{H}), 7.35(\mathrm{td}, J=$ 7.5, $1.1 \mathrm{~Hz}, 1 \mathrm{H}), 7.03(\mathrm{td}, J=7.7,1.6 \mathrm{~Hz}, 1 \mathrm{H}), 5.19(\mathrm{~s}, 2 \mathrm{H}), 4.24(\mathrm{q}, J=7.1 \mathrm{~Hz}, 2 \mathrm{H}), 1.33(\mathrm{t}, J=7.1 \mathrm{~Hz}, 3 \mathrm{H})$. ${ }^{13} \mathrm{C}-\mathrm{NMR}\left(151 \mathrm{MHz}, \mathrm{CDCl}_{3}\right) \delta=154.8,139.5,137.8,130.0,129.3,128.4,98.0,73.0,64.3,14.3$. FTIR (ATR, neat) $\tilde{v}=2994,1734,1438,1381,1365,1282,1259,1013,976,789,744$. HRMS (El, 70 eV) $\mathrm{m} / \mathrm{z}$ $=305.97500$. Calculated for $\left[\mathrm{C}_{10} \mathrm{H}_{11} \mathrm{O}_{3} \mathrm{I}\right]^{+}: \mathrm{m} / z=305.97474 . \mathrm{Mp} T=27-28$.

\section{1-(Chloromethyl)-2-iodobenzene (13)}

In modification of a reported procedure, ${ }^{[18]}$ o-lodobenzylalcohol $(5 \mathrm{a}, 1.87 \mathrm{~g}$, $8.00 \mathrm{mmol})$ was dissolved in dry $\mathrm{DCM}(40 \mathrm{~mL})$ and $\mathrm{SOCl}_{2}(0.73 \mathrm{~mL}, 1.19 \mathrm{~g}$, $10.0 \mathrm{mmol}, 1.25$ eq.) was added dropwise at $0{ }^{\circ} \mathrm{C}$. After complete addition, the cooling bath was removed and the mixture was allowed to warm to rt and stirred over night. Sat. $\mathrm{NaCl}(50 \mathrm{~mL})$ was added, the phases were separated and the

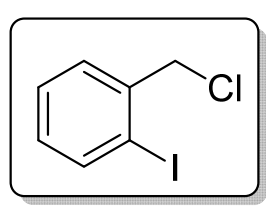
aqueous phase was extracted with DCM $(2 \times 50 \mathrm{~mL})$. The combined organic phases were concentrated under reduced pressure and the residue was purified via column chromatography (silica, cyclohexane) to obtain the product $(\mathbf{1 3}, 1.22 \mathrm{~g}, 4.83 \mathrm{mmol}, 60 \%)$ as a colourless liquid.

${ }^{1} \mathrm{H}-\mathrm{NMR}\left(601 \mathrm{MHz}, \mathrm{CDCl}_{3}\right) \delta=7.87(\mathrm{dd}, J=7.9,0.9 \mathrm{~Hz}, 1 \mathrm{H}), 7.49(\mathrm{dd}, J=7.6,1.4 \mathrm{~Hz}, 1 \mathrm{H}), 7.36(\mathrm{td}, J=$ 7.6, $1.0 \mathrm{~Hz}, 1 \mathrm{H}), 7.01(\mathrm{td}, J=7.7,1.6 \mathrm{~Hz}, 1 \mathrm{H}), 4.68(\mathrm{~s}, 2 \mathrm{H}) .{ }^{13} \mathrm{C}-\mathrm{NMR}\left(151 \mathrm{MHz}, \mathrm{CDCl}_{3}\right) \delta=139.9,139.8$, 130.2, 130.1, 128.8, 99.5, 51.0. FTIR (ATR, neat) $\tilde{v}=3067,2966,1587,1564,1462,1441,1256,1206$, $1013,759,719$. MS (EI, $70 \mathrm{eV}) \mathrm{m} / z=252.0[\mathrm{M}]^{+}$. The analytical data is in accordance with literature data. ${ }^{[18]}$

\subsubsection{Secondary Benzyl Alcohols}

\section{2-lodobenzaldehyde}

o-lodobenzylalcohol (5a, $24.9 \mathrm{~g}, 106 \mathrm{mmol}, 1$ eq.) was dissolved in $\mathrm{CHCl}_{3}(265 \mathrm{~mL})$, activated $\mathrm{MnO}_{2}(92.4 \mathrm{~g}, 1.06 \mathrm{~mol}, 10 \mathrm{eq}$.) was added and the suspension was stirred for $24 \mathrm{~h}$ at $\mathrm{rt}$. The mixture was filtrated over Celite and the filter material was washed with ethyl acetate $(300 \mathrm{~mL})$. After removal of the solvent under reduced

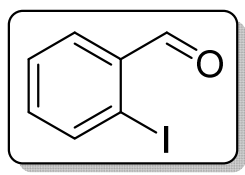
pressure, 2-iodobenzaldehyde $(21.9 \mathrm{~g}, 94.4 \mathrm{mmol}, 89 \%)$ was obtained as a colourless solid.

${ }^{1} \mathrm{H}-\mathrm{NMR}\left(600 \mathrm{MHz}, \mathrm{CDCl}_{3}\right) \delta=10.07(\mathrm{~d}, J=0.7 \mathrm{~Hz}, 1 \mathrm{H}), 7.96$ (dd, $\left.J=7.9,1.1 \mathrm{~Hz}, 1 \mathrm{H}\right), 7.89$ (dd, $J=7.7$, $1.8 \mathrm{~Hz}, 1 \mathrm{H}$ ), $7.47(\mathrm{t}, J=7.5 \mathrm{~Hz}, 1 \mathrm{H}), 7.29$ (ddd, $J=7.9,7.4,1.8 \mathrm{~Hz}, 1 \mathrm{H}) .{ }^{13} \mathrm{C}-\mathrm{NMR}\left(151 \mathrm{MHz}, \mathrm{CDCl}_{3}\right) \delta=$ 196.0, 140.8, 135.7, 135.2, 130.4, 128.9, 100.9. FTIR (ATR, neat) $\tilde{v}=2849,2833,2746,1683,1578$, 
1436, 1388, 1261, 1200, 1015, 748. MS (El, $70 \mathrm{eV}) \mathrm{m} / \mathrm{z}=232.0[\mathrm{M}]^{+}, 203.0[\mathrm{M}-\mathrm{CHO}]^{+} . \mathbf{M p ~} T=34-35^{\circ} \mathrm{C}$. The analytical data is in accordance with literature data. ${ }^{[19]}$

\section{1-(2-lodophenyl)ethan-1-ol (5n)}

To a solution of 2-iodobenzaldehyde ( $3.02 \mathrm{~g}, 13.0 \mathrm{mmol}, 1$ eq.) in dry THF ( $26 \mathrm{~mL})$ at $0{ }^{\circ} \mathrm{C} \mathrm{MeMgBr}(4.8 \mathrm{~mL}, 3 \mathrm{M}$ in THF, $14.3 \mathrm{mmol}, 1.1$ eq.) was added at a rate of $0.8 \mathrm{~mL} / \mathrm{min}$. The mixture was allowed to warm to $\mathrm{rt}$ over night and sat. $\mathrm{NH}_{4} \mathrm{Cl}$ $(10 \mathrm{~mL})$ and $\mathrm{Et}_{2} \mathrm{O}(10 \mathrm{~mL})$ were added. The phases were separated and the aqueous phase was extracted with $\mathrm{Et}_{2} \mathrm{O}(2 \times 10 \mathrm{~mL})$. The combined organic phases

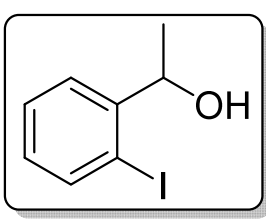
were dried over $\mathrm{Na}_{2} \mathrm{SO}_{4}$, concentrated under reduced pressure and the residue was purified via column chromatography (silica, cyclohexane/ethyl acetate 1:0 $\rightarrow$ 10: 1) to obtain 1-(2-iodophenyl)ethan-1-ol $(5 \mathrm{n}, 2.84 \mathrm{~g}, 8.38 \mathrm{mmol}, 88 \%)$ as a colourless liquid.

${ }^{1} \mathrm{H}-\mathrm{NMR}\left(600 \mathrm{MHz}, \mathrm{CDCl}_{3}\right) \delta=7.80(\mathrm{dd}, J=7.9,1.2 \mathrm{~Hz}, 1 \mathrm{H}), 7.57(\mathrm{dd}, J=7.8,1.7 \mathrm{~Hz}, 1 \mathrm{H}), 7.38(\mathrm{td}, J=$ 7.6, $1.3 \mathrm{~Hz}, 1 \mathrm{H}) 6.97(\mathrm{td}, J=7.7,1.8 \mathrm{~Hz}, 1 \mathrm{H}), 5.08(\mathrm{qd}, J=6.4,3.3 \mathrm{~Hz}, 1 \mathrm{H}), 1.96(\mathrm{~d}, \mathrm{~J}=3.3 \mathrm{~Hz}, 1 \mathrm{H}), 1.47$ (d, $J=6.4 \mathrm{~Hz}, 3 \mathrm{H}) .{ }^{13} \mathrm{C}-\mathrm{NMR}\left(151 \mathrm{MHz}, \mathrm{CDCl}_{3}\right) \delta=147.6,139.5,129.3,128.9,126.5,97.4,73.9,23.9$. FTIR (ATR, neat) $\tilde{v}=3312,3057,2970,2924,1584,1563,1462,1435,1367,1260,1199,1124,1086$, $1067,1045,1007,897,751,720,652 . \mathrm{MS}(\mathrm{EI}, 70 \mathrm{eV}) \mathrm{m} / \mathrm{z}=248.0[\mathrm{M}]^{+}, 233.0\left[\mathrm{M}-\mathrm{CH}_{3}\right]^{+}$. The analytical data is in accordance with literature data. ${ }^{[7]}$

\section{1-(2-lodophenyl)propan-1-ol (50)}

To a suspension of $\mathrm{Mg}$ turnings ( $153 \mathrm{mg}, 6.30 \mathrm{mmol}, 1.4$ eq.) in dry THF ( $1 \mathrm{~mL}$ ) bromoethane $(0.40 \mathrm{~mL}, 5.40 \mathrm{mmol}, 1.2 \mathrm{eq}$.) was slowly added so that the reaction starts and keeps refluxing mildly. After complete formation of the Grignard reagent, dry THF $(3.5 \mathrm{~mL})$ was added and the resulting solution was added to a solution of 2-iodobenzaldehyde $(1.04 \mathrm{~g}, 4.50 \mathrm{mmol}, 1$ eq.) in dry THF $(18 \mathrm{~mL})$ at

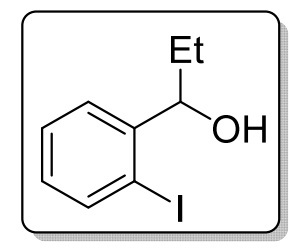
$0{ }^{\circ} \mathrm{C}$ over $15 \mathrm{~min}$. The ice bath was removed and after $30 \mathrm{~min}$. of stirring sat. $\mathrm{NH}_{4} \mathrm{Cl}(7 \mathrm{~mL})$ and $\mathrm{Et}_{2} \mathrm{O}$ $(20 \mathrm{~mL})$ were added. The phases were separated and the aqueous phase was extracted with $\mathrm{Et}_{2} \mathrm{O}(2 \mathrm{x}$ $20 \mathrm{~mL}$ ). The combined organic phases were dried over $\mathrm{Na}_{2} \mathrm{SO}_{4}$ and the solvent was removed under reduced pressure. The residue was purified via column chromatography (silica, cyclohexane/ethyl acetate 10:1) to obtain 1-(2-iodophenyl)propan-1-ol (5o, $560 \mathrm{mg}, 2.13 \mathrm{mmol}, 47 \%$ ) as colourless solid.

${ }^{1} \mathrm{H}-\mathrm{NMR}\left(200 \mathrm{MHz}, \mathrm{CDCl}_{3}\right) \delta=7.80(\mathrm{dd}, J=7.9,1.2 \mathrm{~Hz}, 1 \mathrm{H}), 7.49$ (dd, $\left.J=7.8,1.9 \mathrm{~Hz}, 1 \mathrm{H}\right), 7.36$ (ddd, $J=$ 7.8, 7.2, $1.3 \mathrm{~Hz}, 1 \mathrm{H}), 6.96(\mathrm{td}, J=7.5,1.9 \mathrm{~Hz}, 1 \mathrm{H}), 4.82(\mathrm{dt}, J=7.5,3.8 \mathrm{~Hz}, 1 \mathrm{H}), 2.16(\mathrm{~d}, J=2.9 \mathrm{~Hz}, 1 \mathrm{H})$, $1.96-1.50(\mathrm{~m}, 2 \mathrm{H}), 1.02(\mathrm{t}, J=7.4 \mathrm{~Hz}, 3 \mathrm{H}) .{ }^{13} \mathrm{C}-\mathrm{NMR}\left(50 \mathrm{MHz}, \mathrm{CDCl}_{3}\right) \delta=146.6,139.4,129.2,128.6$, 127.1, 98.0, 78.7, 30.9, 10.3. FTIR (ATR, neat) $\tilde{v}=3284,3059,2968,2918,1560,1460,1426,1351$, 1339, 1277, 1249, 1196, 1126, 1099, 1057, 1008, 972, 870, 809, 750, 722, 672. HRMS (El, 70 eV) m/z $=261.98518$ Calculated for $\mathrm{C}_{9} \mathrm{H}_{11} \mathrm{Ol}^{+\cdot} \mathrm{m} / \mathrm{z}=261.98491$. $\mathrm{Mp} T=40.4-42.4^{\circ} \mathrm{C}$.

\section{1-(2-lodophenyl)-2-methylpropan-1-ol (5p)}

Dry $\mathrm{ZnCl}_{2}$ (636 mg, $4.66 \mathrm{mmol}, 1.01$ eq.) was suspended in dry THF (4.6 mL) and iPrMgCl. LiCl (7.1 mL, 1.3M in THF, $9.20 \mathrm{mmol}, 2$ eq.) was added dropwise over $15 \mathrm{~min}$ and the resulting mixture was stirred for $1 \mathrm{~h}$. After cooling to $0{ }^{\circ} \mathrm{C}$, a solution of 2-iodobenzaldehyde (1.07 g, $4.60 \mathrm{mmol}, 1$ eq.) in dry THF $(4.6 \mathrm{~mL})$ was added over $30 \mathrm{~min}$. The reaction was warmed to rt over night and sat. $\mathrm{NH}_{4} \mathrm{Cl}(5 \mathrm{~mL})$

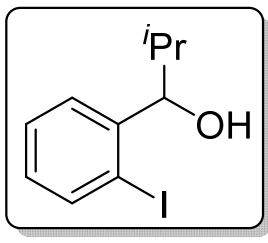
and EtOAc $(5 \mathrm{~mL})$ were added. The phases were separated and the aqueous phase was extracted with EtOAc $(2 \times 5 \mathrm{~mL})$. The combined organic phases were dried over $\mathrm{Na}_{2} \mathrm{SO}_{4}$ and concentrated under reduced pressure to obtain 1-(2-iodophenyl)-2-methylpropan-1-ol (5p, $1.19 \mathrm{~g}, 4.31 \mathrm{mmol}, 94 \%$ ) as a colourless liquid. 
${ }^{1} \mathrm{H}-\mathrm{NMR}\left(200 \mathrm{MHz}, \mathrm{CDCl}_{3}\right) \delta=7.79(\mathrm{dd}, J=7.9,1.2 \mathrm{~Hz}, 1 \mathrm{H}), 7.41(\mathrm{dd}, J=7.8,2.1 \mathrm{~Hz}, 1 \mathrm{H}), 7.38-7.28$ $(\mathrm{m}, 1 \mathrm{H}), 6.94(\mathrm{ddd}, J=7.8,6.9,2.1 \mathrm{~Hz}, 1 \mathrm{H}), 4.67(\mathrm{~d}, J=5.6 \mathrm{~Hz}, 1 \mathrm{H}), 2.40(\mathrm{~s}, 1 \mathrm{H}), 2.02(\mathrm{dq}, J=13.4,6.7$ $\mathrm{Hz}, 1 \mathrm{H}), 0.97(\mathrm{~d}, J=2.3 \mathrm{~Hz}, 3 \mathrm{H}), 0.93(\mathrm{~d}, J=2.1 \mathrm{~Hz}, 3 \mathrm{H}) .{ }^{13} \mathrm{C}-\mathrm{NMR}\left(50 \mathrm{MHz}, \mathrm{CDCl}_{3}\right) \delta=145.8,139.3$, 129.1, 128.3, 128.0, 98.7, 81.8, 34.1, 19.7, 16.6. FTIR (ATR, neat) $\tilde{v}=3370,3058,2958,2871,1584$, 1562, 1460, 1384, 1364, 1197, 1173, 1132, 1094, 1003, 943, 747. HRMS (EI, 70 eV) $\mathrm{m} / \mathrm{z}=276.00047$. Calculated for $\mathrm{C}_{10} \mathrm{H}_{13} \mathrm{Ol} \mathrm{O}^{+\cdot} \mathrm{m} / z=276.00056$. MS (EI, $70 \mathrm{eV}$ ) $\mathrm{m} / z=276.0[\mathrm{M}]^{+}, 233.0\left[\mathrm{M}-\mathrm{C}_{3} \mathrm{H}_{7}\right]^{+}$. The analytical data is in accordance with literature data. ${ }^{[7]}$

\section{1-(5-Bromo-2-iodophenyl)ethan-1-ol (5q)}

(5-Bromo-2-iodophenyl)methanol (5b, $939 \mathrm{mg}, 3.00 \mathrm{mmol}, 1.00$ eq.) was dissolved in $\mathrm{CH}_{3} \mathrm{Cl}(12 \mathrm{~mL})$, activated $\mathrm{MnO}_{2}(5.22 \mathrm{~g}, 60.0 \mathrm{mmol}, 20.0$ eq.) was added and the resulting suspension was stirred for $24 \mathrm{~h}$. The mixture was filtered over silica, the filter material washed with EtOAc $(100 \mathrm{~mL})$ and the organic phase was concentrated under reduced pressure to obtain 5-bromo-2iodobenzaldehyde (882 $\mathrm{mg}, 2.84 \mathrm{mmol}, 95 \%$ ) as a colourless solid.

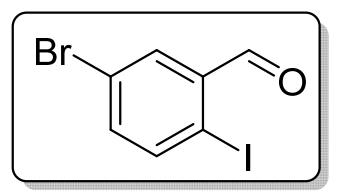

${ }^{1} \mathrm{H}-\mathrm{NMR}\left(360 \mathrm{MHz}, \mathrm{CDCl}_{3}\right) \delta=9.98(\mathrm{~s}, 1 \mathrm{H}), 7.98(\mathrm{~d}, J=2.5 \mathrm{~Hz}, 1 \mathrm{H}), 7.80(\mathrm{~d}, J=8.4 \mathrm{~Hz}, 1 \mathrm{H}), 7.41(\mathrm{dd}, J=$ 8.4, $2.5 \mathrm{~Hz}, 1 \mathrm{H}) .{ }^{13} \mathrm{C}-\mathrm{NMR}\left(91 \mathrm{MHz}, \mathrm{CDCl}_{3}\right) \delta=194.3,141.8,138.2,136.2,133.0,123.4,98.3$. FTIR (ATR, neat) $\tilde{v}=3071,2849,1682,1564,1477,1449,1374,1248,1187,1007,823$. MS (El, 70 eV) $\mathrm{m} / \mathbf{z}=309.9$ $[\mathrm{M}]^{+}, 280.8[\mathrm{M}-\mathrm{CHO}]^{+}, 181.9[\mathrm{M}-\mathrm{HI}]^{+} . \mathrm{Mp} T=91.5-92.5^{\circ} \mathrm{C}$. The analytical data is in accordance with literature data. ${ }^{[9]}$

5-Bromo-2-iodobenzaldehyde ( $622 \mathrm{mg}, 2.00 \mathrm{mmol}, 1$ eq.) was dissolved in dry THF (10 mL), cooled to $-10^{\circ} \mathrm{C}$ and $\mathrm{MeMgBr}(0.80 \mathrm{~mL}, 3 \mathrm{M}$ in THF, $2.40 \mathrm{mmol}$, 1.2 eq.) was added dropwise over $10 \mathrm{~min}$. The cooling bath was removed and the mixture stirred for $30 \mathrm{~min}$. Sat. $\mathrm{NH}_{4} \mathrm{Cl}(10 \mathrm{~mL})$ and $\mathrm{Et}_{2} \mathrm{O}(10 \mathrm{~mL})$ were added, the phases separated and the aqueous phase was extracted with $\mathrm{Et}_{2} \mathrm{O}$

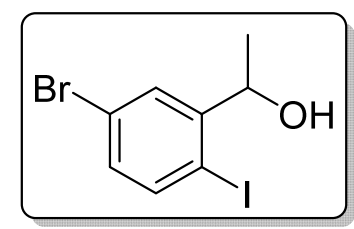
$(2 \times 10 \mathrm{~mL})$. The combined organic phases were dried over $\mathrm{Na}_{2} \mathrm{SO}_{4}$ and concentrated under reduced pressure to obtain 1-(5-bromo-2-iodophenyl)ethan-1-ol (5q, $644 \mathrm{mg}, 1.97 \mathrm{mmol}, 98 \%$ ) as a colourless solid.

${ }^{1} \mathrm{H}-\mathrm{NMR}\left(360 \mathrm{MHz}, \mathrm{CDCl}_{3}\right) \delta=7.68(\mathrm{~d}, J=2.4 \mathrm{~Hz}, 1 \mathrm{H}), 7.61$ (d, $\left.J=8.4 \mathrm{~Hz}, 1 \mathrm{H}\right), 7.09$ (dd, $J=8.4,2.5 \mathrm{~Hz}$, $1 \mathrm{H}), 4.98(\mathrm{dq}, J=6.2,1.4 \mathrm{~Hz}, 1 \mathrm{H}), 2.33(\mathrm{~d}, J=1.8 \mathrm{~Hz}, 1 \mathrm{H}), 1.42(\mathrm{~d}, J=6.4 \mathrm{~Hz}, 3 \mathrm{H}) .{ }^{13} \mathrm{C}-\mathrm{NMR}(91 \mathrm{MHz}$, $\left.\mathrm{CDCl}_{3}\right) \delta=149.5,140.4,132.1,129.6,123.2,94.7,73.3,23.7$. FTIR (ATR, neat) $\tilde{v}=3292,2978,1439$, $1367,1185,1081,1005,900,800 . \mathrm{MS}(\mathrm{El}, 70 \mathrm{eV}) \mathrm{m} / \mathrm{z}=325.9[\mathrm{M}]^{+}, 310.9[\mathrm{M}-\mathrm{Me}]^{+} . \mathrm{Mp} T=57-58^{\circ} \mathrm{C}$

\section{(2-lodophenyl)(phenyl)methanol (5k)}

To a suspension of $\mathrm{Mg}$ turnings ( $115 \mathrm{mg}, 4.73 \mathrm{mmol}, 1.05$ eq.) in dry $\mathrm{Et}_{2} \mathrm{O}$ ( $1 \mathrm{~mL}$ ) iodobenzene $(0.56 \mathrm{~mL}, 4.95 \mathrm{mmol}, 1.1$ eq.) was slowly added so that the reaction starts and keeps refluxing mildly. After complete formation of the Grignard reagent, dry $\mathrm{Et}_{2} \mathrm{O}(5 \mathrm{~mL})$ was added and the resulting solution was added to a solution of 2-iodobenzaldehyde ( $1.04 \mathrm{~g}, 4.50 \mathrm{mmol}, 1$ eq.) in dry $\mathrm{Et}_{2} \mathrm{O}(10 \mathrm{~mL})$ at

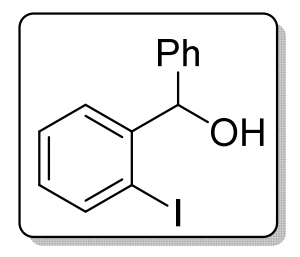
$0{ }^{\circ} \mathrm{C}$ over $15 \mathrm{~min}$. The ice bath was removed and after $30 \mathrm{~min}$. of stirring sat. $\mathrm{NH}_{4} \mathrm{Cl}(10 \mathrm{~mL})$ and EtOAC $(10 \mathrm{~mL})$ were added. The phases were separated and the aqueous phase was extracted with $\mathrm{Et}_{2} \mathrm{O}(3 \mathrm{x}$ $10 \mathrm{~mL}$ ). The combined organic phases were dried over $\mathrm{Na}_{2} \mathrm{SO}_{4}$ and the solvent was removed under reduced pressure. The residue was purified via column chromatography (silica, cyclohexane/ethyl acetate 20:1) to obtain (2-iodophenyl)(phenyl)methanol (5k, $829 \mathrm{mg}, 2.67 \mathrm{mmol}, 59 \%$ ) as colourless liquid. 
${ }^{1} \mathrm{H}-\mathrm{NMR}\left(200 \mathrm{Mhz}, \mathrm{CDCl}_{3}\right) \delta=7.84(\mathrm{dd}, J=7.9,1.2 \mathrm{~Hz}, 1 \mathrm{H}), 7.53(\mathrm{dd}, J=7.8,1.8 \mathrm{~Hz}, 1 \mathrm{H}), 7.49-7.24(\mathrm{~m}$, $6 \mathrm{H}), 7.00(\mathrm{td}, J=7.7,1.8 \mathrm{~Hz}, 1 \mathrm{H}), 6.06(\mathrm{~d}, J=3.6 \mathrm{~Hz}, 1 \mathrm{H}), 2.49$ (dd, $J=3.7,1.1 \mathrm{~Hz}, 1 \mathrm{H}) .{ }^{13} \mathrm{C}-\mathrm{NMR}(50 \mathrm{MHz}$, $\left.\mathrm{CDCl}_{3}\right) \delta=145.4,142.2,139.6,129.5,128.7,128.6,128.5,127.9,127.3,98.8,79.1$. FTIR (ATR, neat) $\tilde{v}$ = 3320, 3059, 3028, 1584, 1562, 1493, 1452, 1435, 1181, 1114, 1077, 1006, 916, 849, 918, 746, 697, 669. MS (EI, $70 \mathbf{~ e V}) \mathrm{m} / \mathrm{z}=310.0[\mathrm{M}]^{+.}$. The analytical data is in accordance with literature data. ${ }^{[20]}$

\section{(3,5-Bis(trifluoromethyl)phenyl)(2-iodophenyl)methanol (5I)}

3,5-Bis(trifluoromethyl)bromobenzene $(806 \mathrm{mg}, 2.75 \mathrm{mmol}, 1.1$ eq.) was dissolved in dry THF $(20 \mathrm{~mL})$ and cooled to $-78^{\circ} \mathrm{C}$. $n$ BuLi $(1.1 \mathrm{~mL}, 2.5 \mathrm{M}$ in hexanes, $2.75 \mathrm{mmol}, 1.1$ eq.) was added dropwise over $10 \mathrm{~min}$ and the solution was stirred for $10 \mathrm{~min}$. 2-lodobenzaldehyde $(580 \mathrm{mg}, 2.50 \mathrm{mmol}$, 1.00 eq.) in dry THF $(10 \mathrm{~mL})$ was added dropwise over $30 \mathrm{~min}$. via syringe pump. After complete addition the mixture was slowly warmed to rt. Sat. $\mathrm{NH}_{4} \mathrm{Cl}(50 \mathrm{~mL})$ and $\mathrm{Et}_{2} \mathrm{O}(50 \mathrm{~mL})$ were added, the phases were separated and the aqueous phase was extracted with $\mathrm{Et}_{2} \mathrm{O}(2 \times 50 \mathrm{~mL})$. The combined organic

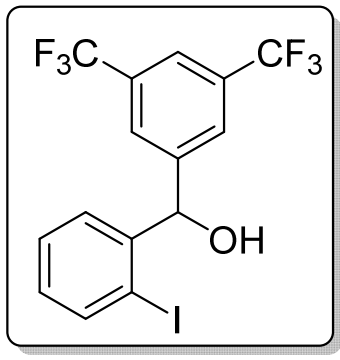
phases were washed with brine $(20 \mathrm{~mL})$, dried over $\mathrm{Na}_{2} \mathrm{SO}_{4}$ and concentrated under reduced pressure. The residue was purified via column chromatography (cyclohexane/ethyl acetate $30: 1$ ) to obtain $(3,5-$ (bis(trifluoromethyl)phenyl)(2-iodophenyl)methanol (5l, $640 \mathrm{mg}, 1.44 \mathrm{mmol}, 57 \%$ ) as a yellow oil.

${ }^{1} \mathrm{H}-\mathrm{NMR}\left(600 \mathrm{MHz}, \mathrm{CDCl}_{3}\right) \delta=7.91(\mathrm{~s}, 2 \mathrm{H}), 7.89(\mathrm{~d}, J=8.0 \mathrm{~Hz}, 1 \mathrm{H}), 7.81(\mathrm{~s}, 1 \mathrm{H}), 7.40(\mathrm{t}, J=7.4 \mathrm{~Hz}, 1 \mathrm{H})$, $7.37(\mathrm{~d}, J=7.7 \mathrm{~Hz}, 1 \mathrm{H}), 7.06(\mathrm{t}, J=7.5 \mathrm{~Hz}, 1 \mathrm{H}), 6.22(\mathrm{~d}, J=3.1 \mathrm{~Hz}, 1 \mathrm{H}), 2.54(\mathrm{~d}, J=3.4 \mathrm{~Hz}, 1 \mathrm{H}) .{ }^{13} \mathrm{C}-\mathrm{NMR}$ $\left(151 \mathrm{MHz}, \mathrm{CDCl}_{3}\right) \delta=144.6,144.2,139.9,131.7$ (q, $\left.J=33.3 \mathrm{~Hz}\right), 130.3,129.2,128.4,127.5-126.4(\mathrm{~m})$, $123.3(\mathrm{q}, J=272.9 \mathrm{~Hz}$ ), $121.7-121.6(\mathrm{~m}), 98.6,77.9$. FTIR (ATR, neat) $\tilde{v}=3289,1374,1274,1165$,

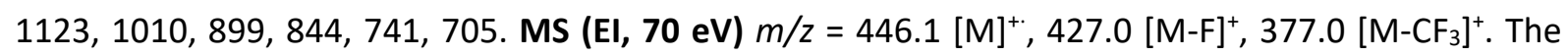
analytical data is in accordance with literature data. ${ }^{[21]}$

\section{(5-Chloro-2-iodophenyl)(2-fluorophenyl)methanol (5m)}

Following GP1a, (2-amino-5-chlorophenyl)(2-fluorophenyl)methanone ( $1.25 \mathrm{~g}, 5.00 \mathrm{mmol}, 1.00$ eq.) and $\mathrm{Ts} \mathrm{OH} \cdot \mathrm{H}_{2} \mathrm{O}(2.85 \mathrm{~g}, 15.0 \mathrm{mmol}, 3.00$ eq.) were suspended in Acetonitrile $(40 \mathrm{~mL})$. The mixture was cooled to $10-15$ ${ }^{\circ} \mathrm{C}$ and a solution of $\mathrm{NaNO}_{2}(690 \mathrm{mg}, 10.0 \mathrm{mmol}, 2.00$ eq.) and $\mathrm{KI}(2.08 \mathrm{~g}$, $12.5 \mathrm{mmol}, 2.50$ eq.) in $\mathrm{H}_{2} \mathrm{O}(6 \mathrm{~mL})$ was added dropwise over $1 \mathrm{~h}$. After complete addition, the resulting dark coloured mixture was allowed to stir

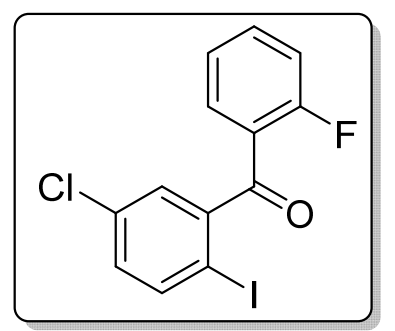
for another $1 \mathrm{~h}$ and then $1 \mathrm{~N} \mathrm{HCl}(25 \mathrm{~mL})$ and EtOAc $(50 \mathrm{~mL})$ were added. The phases were separated and the aqueous phases was extracted with EtOAc $(2 \times 50 \mathrm{~mL})$. The combined organic phases were washed with water $(25 \mathrm{~mL}), \mathrm{Na}_{2} \mathrm{~S}_{2} \mathrm{O}_{3}(10 \% \mathrm{w} / \mathrm{w}, 25 \mathrm{~mL})$ and brine $(20 \mathrm{~mL})$, dried over $\mathrm{Na}_{2} \mathrm{SO}_{4}$ and concentrated under reduced pressure. The residue was purified by column chromatography (cyclohexane/ethyl acetate 50:1) to obtain (5-chloro-2-iodophenyl)(2-fluorophenyl)methanone (1.37 g, $3.81 \mathrm{mmol}, 76 \%)$.

${ }^{1} \mathrm{H}-\mathrm{NMR}\left(360 \mathrm{MHz}, \mathrm{CDCl}_{3}\right) \delta=7.82(\mathrm{~d}, J=8.4 \mathrm{~Hz}, 1 \mathrm{H}), 7.77(\mathrm{td}, J=7.6,7.6,1.7 \mathrm{~Hz}, 1 \mathrm{H}), 7.64-7.53(\mathrm{~m}$, $1 \mathrm{H}), 7.30(\mathrm{~d}, J=2.4 \mathrm{~Hz}, 1 \mathrm{H}), 7.27(\mathrm{t}, J=7.6,7.6 \mathrm{~Hz}, 1 \mathrm{H}), 7.15(\mathrm{dd}, J=8.4,2.5 \mathrm{~Hz}, 1 \mathrm{H}), 7.13-7.08(\mathrm{~m}$, 1H). ${ }^{13} \mathrm{C}-\mathrm{NMR}\left(91 \mathrm{MHz}, \mathrm{CDCl}_{3}\right) \delta=192.6,161.7(\mathrm{~d}, J=259 \mathrm{~Hz}), 146.3,141.0,135.6(\mathrm{~d}, J=9.0 \mathrm{~Hz}), 134.6$, 131.9, 131.6, $128.5(\mathrm{~d}, J=1.1 \mathrm{~Hz}), 124.6(\mathrm{~d}, J=3.6 \mathrm{~Hz}), 124.4(\mathrm{~d}, J=10.2 \mathrm{~Hz}), 116.8(\mathrm{~d}, J=22.0 \mathrm{~Hz}), 88.7$ (d, $J=1.2 \mathrm{~Hz}$ ). FTIR (ATR, neat) $\tilde{v}=3071,1669,1606,1478,1450,1372,1288,1096,944,750$. HRMS (EI, $70 \mathrm{eV}) \mathrm{m} / \mathrm{z}=359.92118[\mathrm{M}]^{+}$. Calculated for $\left[\mathrm{C}_{13} \mathrm{H}_{7} \mathrm{OCIFI}\right]^{+}: \mathrm{m} / z=359.92087 . \mathbf{M p} T=90-91$. 
(5-Chloro-2-iodophenyl)(2-fluorophenyl)methanone $(721 \mathrm{mg}, 2.00 \mathrm{mmol}$, 1.00 eq.) was dissolved in $\mathrm{MeOH}(4 \mathrm{~mL})$ and at $0{ }^{\circ} \mathrm{C} \mathrm{NaBH} 4(37.8 \mathrm{mg}$, $1.00 \mathrm{mmol}, 0.500$ eq.) was added. After stirring for $30 \mathrm{~min}^{\mathrm{Et}} \mathrm{O}_{2}(20 \mathrm{~mL})$ and brine $(5 \mathrm{~mL})$ were added. The phases were separated, the aqueous phase was extracted with $\mathrm{Et}_{2} \mathrm{O}(3 \times 20 \mathrm{~mL})$ and the combined organic phases were dried over $\mathrm{Na}_{2} \mathrm{SO}_{4}$ and concentrated under reduced pressure, to obtain (5chloro-2-iodophenyl)(2-fluorophenyl)methanol $(5 \mathrm{~m}, 712 \mathrm{mg}, 1.96 \mathrm{mmol}$,

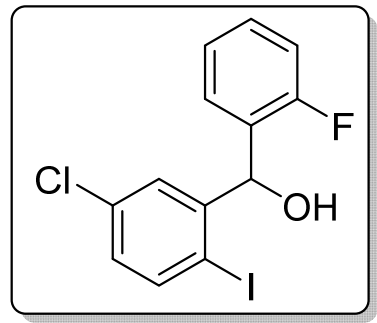
$98 \%)$ as a slightly yellow oil

${ }^{1} \mathrm{H}-\mathrm{NMR}\left(200 \mathrm{MHz}, \mathrm{CDCl}_{3}\right) \delta=7.75(\mathrm{~d}, J=8.4 \mathrm{~Hz}, 1 \mathrm{H}), 7.55(\mathrm{~d}, J=2.6 \mathrm{~Hz}, 1 \mathrm{H}), 7.42-7.26(\mathrm{~m}, 1 \mathrm{H}), 7.23$ $-7.07(\mathrm{~m}, 3 \mathrm{H}), 7.03(\mathrm{dd}, J=8.4,2.6 \mathrm{~Hz}, 1 \mathrm{H}), 6.20(\mathrm{~d}, J=3.5 \mathrm{~Hz}, 1 \mathrm{H}), 2.83(\mathrm{~d}, J=3.7 \mathrm{~Hz}, 1 \mathrm{H}) .{ }^{13} \mathrm{C}-\mathrm{NMR}$ $\left(50 \mathrm{MHz}, \mathrm{CDCl}_{3}\right) \delta=160.6(\mathrm{~d}, J=248.0 \mathrm{~Hz}), 145.6,140.5,134.9,129.9(\mathrm{~d}, J=8.3 \mathrm{~Hz}$ ), 129.6, 128.5, 128.4, 128.1 (d, $J=16.1 \mathrm{~Hz}), 124.3(\mathrm{~d}, J=3.5 \mathrm{~Hz}), 115.5$ (d, $J=21.4 \mathrm{~Hz}), 95.2,73.0$ (d, $J=3.2 \mathrm{~Hz})$. FTIR (ATR, neat) $\tilde{v}=3264,1614,1586,1487,1452,1385,1227,1093,1007,900,807,754$. HRMS (EI, $70 \mathrm{eV}$ ) $\mathrm{m} / \mathrm{z}=361.93641[\mathrm{M}]^{++}$. Calculated for $\left[\mathrm{C}_{13} \mathrm{H}_{9} \mathrm{OClFI}\right]^{+}: \mathrm{m} / \mathrm{z}=361.93652$.

\section{Substrate synthesis}

\subsection{General Procedures for the One-Pot Reaction Utilizing Primary Benzyl Alcohols and Secondary Aromatic Benzyl Alcohols (OPP1)}

\subsubsection{Standard conditions (OPP1a)}

The arene 6 (600 $\mu \mathrm{mol}-6.00 \mathrm{mmol}, 3.00-30.0$ eq.) was dissolved in DCM $(0.5 \mathrm{~mL})$ in a screw cap vial, cooled to $0{ }^{\circ} \mathrm{C}$ and $\mathrm{TfOH}(87.8 \mu \mathrm{L}, 150 \mathrm{mg}, 1.00 \mathrm{mmol}, 5.00$ eq.) was added. To this solution, o-iodobenzylalcohol derivative 5 ( $200 \mu \mathrm{mol}, 1$ eq.) in $\mathrm{DCM}(0.5 \mathrm{~mL})$ was added by slowly rinsing it down the wall of the glass vial within one minute. If the alcohol $\mathbf{5}$ could not be dissolved in DCM it was added as solid in one portion. After complete consumption of 5, MeCN $(1 \mathrm{~mL})$ and $\mathrm{H}_{2} \mathrm{SO}_{4}(53.3 \mu \mathrm{L}, 98.1 \mu \mathrm{g}$, $1.00 \mathrm{mmol}, 5.00$ eq.) were added. Then $\mathrm{Oxone}^{\circledR}(123 \mathrm{mg}, 200 \mu \mathrm{mol}, 1.00$ eq.) was added in one portion, the vial was tightly closed and the suspension was stirred at room temperature for the indicated time. After completion of the reaction DCM $(5 \mathrm{~mL})$ and water $(5 \mathrm{~mL})$ were added and the phases were separated. The aqueous phase was extracted with DCM $(2 \times 5 \mathrm{~mL})$ and the combined organic phases were dried over $\mathrm{Na}_{2} \mathrm{SO}_{4}$ and concentrated under reduced pressure. The residue was washed with EtOAc/Cy (1:4) $(2 \times 2 \mathrm{~mL})$, dried under reduced pressure and then washed with water (2 $\times 2 \mathrm{~mL}$ ) to obtain the product after lyophilization.

\subsubsection{Conditions for benzyl alcohols with electron withdrawing groups (OPP1b)}

With benzyl alcohols carrying electron withdrawing groups, the oxidation may not be completed under the conditions described in OPP1a. In this case $0.2 \mathrm{~mL}$ of 1,1,1,3,3,3-hexyfluoroisopropanol (HFIP) is added just before addition of Oxone. 


\subsubsection{Conditions for benzyl alcohols with electron withdrawing groups (OPP1c)}

When utilizing benzylalcohols with more strongly electron withdrawing groups, one equivalent of Oxone ${ }^{\circledR}$ may not be sufficient to complete the oxidation in the second step of the reaction, i.e. for example with $\mathrm{NO}_{2}$-groups. In these cases, after $16 \mathrm{~h}$ reaction time in the oxidation step a second equivalent of $O x e^{\circledR}(123 \mathrm{mg}, 200 \mu \mathrm{mol}, 1.00$ eq.) was added and the mixture was stirred for another $24 \mathrm{~h}$.

\subsection{Substrates from Primary Benzyl Alcohols and Secondary Aromatic Benzyl Alcohols}

\section{$10 H$-Dibenzo[b,e]iodinin-5-ium trifluoromethanesulfonate $(7 a)$}

Following OPP1a, the reaction of 0 -iodobenzylalcohol $(5 \mathbf{a}, 46.8 \mathrm{mg})$ with benzene (178 $\mu \mathrm{L}, 156 \mathrm{mg}, 2.00 \mathrm{mmol}, 10$ eq.) gave after $5 \mathrm{~min}$ for the first reaction step and $2 \mathrm{~h}$ for the second reaction step the product $7 \mathrm{a}(78.6 \mathrm{mg}$, $0.178 \mathrm{mmol}, 89 \%$ ) as a colourless solid.

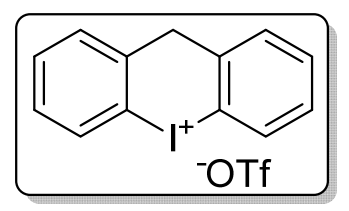

\section{Upscaled reaction:}

Benzene $(6 \mathrm{a}, 4.44 \mathrm{~mL}, 3.91 \mathrm{~g}, 50.0 \mathrm{mmol}, 10.0$ eq.) was dissolved in DCM (12.5 mL) in a pressure vial, cooled to $0{ }^{\circ} \mathrm{C}$ and $\mathrm{TfOH}(2.19 \mathrm{~mL}, 3.75 \mathrm{~g}, 25.0 \mathrm{mmol}, 5.00$ eq.) was added. To this solution, o-iodobenzylalcohol (1.17 g, $5.00 \mathrm{mmol}, 1.00$ eq.) in DCM $(12.5 \mathrm{~mL})$ was added dropwise via a syringe within $5 \mathrm{~min}$. After complete consumption of the substrate $(5 \mathrm{~min}), \mathrm{MeCN}(25 \mathrm{~mL})$ and $\mathrm{H}_{2} \mathrm{SO}_{4}(1.33 \mathrm{~mL}$, $2.45 \mathrm{~g}, 25.0 \mathrm{mmol}, 5.00 \mathrm{eq}$.) were added. Then Oxone ( $3.07 \mathrm{~g}, 5.0 \mathrm{mmol}, 1.00 \mathrm{eq}$.) was added in one portion, the vial was tightly closed and the suspension was stirred at room temperature for $2 \mathrm{~h}$. After completion of the reaction, DCM $(25 \mathrm{~mL})$ and water $(25 \mathrm{~mL})$ were added and the phases were separated. The aqueous phase was extracted with DCM $(2 \times 25 \mathrm{~mL})$ and the combined organic phases were dried over $\mathrm{Na}_{2} \mathrm{SO}_{4}$ and concentrated under reduced pressure. The residue was washed with an EtOAc/Cy 1:4 mixture $(3 \times 10 \mathrm{~mL})$, and dried under reduced pressure to obtain the product $(2.06 \mathrm{~g}$, $4.66 \mathrm{mmol}, 93 \%)$ as a colourless solid.

At this stage, the product was already sufficiently pure as estimated by NMR. To test the general procedure in a larger scale, the product was washed with water $(3 \times 10 \mathrm{~mL})$ and lyophilized. To remove the water completely, the product had to be heated to $50^{\circ} \mathrm{C}$ for $1 \mathrm{~h}$ at a pressure of approx. $0.1 \mathrm{mbar}$ on the larger scale. No significant increase in purity was observed and the product was again obtained as a colourless solid $(1.94 \mathrm{~g}, 4.39 \mathrm{mmol}, 88 \%)$.

\section{Reaction with derivatives of $o$-iodobenzylalcohol:}

Following OPP1a, the reaction of $o$-iodobenzylacetate $(10,55.2 \mathrm{mg}$ ) with benzene $(178 \mu \mathrm{L}, 156 \mathrm{mg}$, $2.00 \mathrm{mmol}, 10$ eq.) gave after $5 \mathrm{~min}$ for the first reaction step and $2 \mathrm{~h}$ for the second reaction step the product $7 \mathrm{a}(76.6 \mathrm{mg}, 0.173 \mathrm{mmol}, 87 \%)$ as a colourless solid.

Following OPP1a, the reaction of 1-iodo-2-(methoxymethyl)benzene $(\mathbf{1 1}, 49.6 \mathrm{mg})$ with benzene (178 $\mu \mathrm{L}, 156 \mathrm{mg}, 2.00 \mathrm{mmol}, 10$ eq.) gave after $5 \mathrm{~min}$ for the first reaction step and $2 \mathrm{~h}$ for the second reaction step the product $7 \mathrm{a}(70.2 \mathrm{mg}, 0.159 \mathrm{mmol}, 79 \%)$ as a colourless solid. 
Following OPP1a, the reaction of ethyl (2-iodobenzyl) carbonate $(12,61.2 \mathrm{mg})$ with benzene $(178 \mu \mathrm{L}$, $156 \mathrm{mg}, 2.00 \mathrm{mmol}, 10$ eq.) gave after $5 \mathrm{~min}$ for the first reaction step and $2 \mathrm{~h}$ for the second reaction step the product $7 \mathrm{a}(78.3 \mathrm{mg}, 0.177 \mathrm{mmol}, 89 \%)$ as a colourless solid.

Following OPP1a, the reaction of o-iodobenzylchloride $(13,50.5 \mathrm{mg})$ with benzene $(178 \mu \mathrm{L}, 156 \mathrm{mg}$, $2.00 \mathrm{mmol}, 10$ eq.) gave after $5 \mathrm{~min}$. for the first reaction step and $2 \mathrm{~h}$ for the second reaction step a complex product mixture that contained $7 \mathrm{a}$ in less than $10 \%$ yield.

${ }^{1} \mathrm{H}-\mathrm{NMR}\left(601 \mathrm{MHz}\right.$, DMSO- $\left.d_{6}\right) \delta=8.10(\mathrm{dd}, J=8.1,1.2 \mathrm{~Hz}, 2 \mathrm{H}), 7.81(\mathrm{dd}, J=7.6,1.6 \mathrm{~Hz}, 2 \mathrm{H}), 7.61(\mathrm{td}, J$ $=7.5,1.2 \mathrm{~Hz}, 2 \mathrm{H}), 7.43(\mathrm{td}, J=7.7,1.6 \mathrm{~Hz}, 2 \mathrm{H}), 4.32(\mathrm{~s}, 2 \mathrm{H}) .{ }^{13} \mathrm{C}-\mathrm{NMR}\left(151 \mathrm{MHz}\right.$, DMSO- $\left.d_{6}\right) \delta=138.9$, $133.7,131.8,130.5,129.1,120.7$ (q, $J=322.7 \mathrm{~Hz}), 116.0,45.7 .{ }^{19} \mathrm{~F}-\mathrm{NMR}\left(565 \mathrm{MHz}\right.$, DMSO- $\left.d_{6}\right) \delta=-77.7$. FTIR (ATR, neat) $\tilde{v}=3094,3059,1457,1441,1426,1237,1220,1152,1023,751$. HRMS (ESI ${ }^{+}$, MeOH) $m / z=292.98209[\mathrm{M}-\mathrm{OTf}]^{+}$. Calculated for $\left[\mathrm{C}_{13} \mathrm{H}_{10} \mathrm{l}\right]^{++}: \mathrm{m} / \mathrm{z}=292.98217 . \mathrm{Mp} \mathrm{T}=219-221^{\circ} \mathrm{C}$ (decomp.). The analytical data is in accordance with literature data. ${ }^{[22]}$

\section{2-Bromo-10H-dibenzo[b,e]iodinin-5-ium trifluoromethanesulfonate (7b)}

Following OPP1b, the reaction of (5-bromo-2-iodophenyl)methanol (5b, $62.6 \mathrm{mg}$ ) with benzene ( $178 \mu \mathrm{L}, 156 \mathrm{mg}, 2.00 \mathrm{mmol}, 10$ eq.) gave after $15 \mathrm{~min}$ for the first reaction step and $16 \mathrm{~h}$ for the second reaction step the product $7 \mathrm{~b}(88.3 \mathrm{mg}, 0.169 \mathrm{mmol}, 85 \%)$ as a colourless solid.

${ }^{1} \mathrm{H}-\mathrm{NMR}\left(601 \mathrm{MHz}\right.$, DMSO- $\left.\boldsymbol{d}_{6}\right) \delta=8.11-8.07(\mathrm{~m}, 2 \mathrm{H}), 8.01(\mathrm{~d}, J=8.6 \mathrm{~Hz}$,

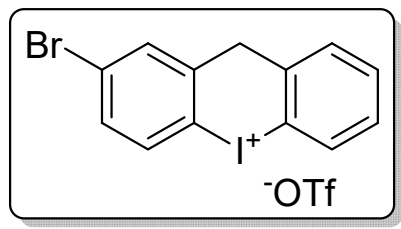
$1 \mathrm{H}), 7.76(\mathrm{dd}, J=7.6,1.7 \mathrm{~Hz}, 1 \mathrm{H}), 7.66(\mathrm{dd}, J=8.6,2.4 \mathrm{~Hz}, 1 \mathrm{H}), 7.62(\mathrm{td}, J=7.5,1.2 \mathrm{~Hz}, 1 \mathrm{H}), 7.44(\mathrm{td}, J$ $=7.7,1.6 \mathrm{~Hz}, 1 \mathrm{H}), 4.33(\mathrm{~s}, 2 \mathrm{H}) .{ }^{13} \mathrm{C}-\mathrm{NMR}\left(151 \mathrm{MHz}\right.$, DMSO-d $\left.d_{6}\right) \delta=141.6,138.4,135.3,133.6,132.9$, 131.9, 131.7, 130.7, 129.2, 125.4, 120.7 (q, $J=322.3 \mathrm{~Hz}$ ), 116.2, 114.9, 45.0. ${ }^{19} \mathrm{~F}-\mathrm{NMR}$ (565 MHz, DMSO$\left.\boldsymbol{d}_{6}\right) \delta=-77.7$. FTIR (ATR, neat) $\tilde{v}=3094,1457,1392,1273,1231,1220,1170,1021,992,755$. HRMS (ESI $\left.{ }^{+}, \mathbf{M e O H}\right) m / z=370.89224[\mathrm{M}-\mathrm{OTf}]^{+}$. Calculated for $\left[\mathrm{C}_{13} \mathrm{H} \mathrm{H}_{9} \mathrm{Br}\right]^{+}: \mathrm{m} / z=370.89268$. Mp $T=210-$ 215.

\section{2-Nitro-10H-dibenzo[b,e]iodinin-5-ium trifluoromethanesulfonate (7c)}

Following OPP1c, the reaction of (5-nitro-2-iodophenyl)methanol (5c, $55.8 \mathrm{mg}$ ) with benzene ( $178 \mu \mathrm{L}, 156 \mathrm{mg}, 2.00 \mathrm{mmol}, 10$ eq.) gave after $5 \mathrm{~h}$ for the first reaction step and a total of $40 \mathrm{~h}$ for the second reaction step the product 7c $(81.7 \mathrm{mg}, 0.168 \mathrm{mmol}, 84 \%)$ as a colourless solid.

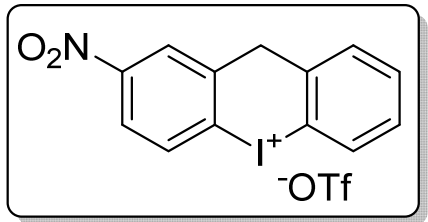

${ }^{1} \mathrm{H}-\mathrm{NMR}\left(601 \mathrm{MHz}\right.$, DMSO- $\left.d_{6}\right) \delta=8.67(\mathrm{~d}, J=2.7 \mathrm{~Hz}, 1 \mathrm{H}), 8.36(\mathrm{~d}, J=8.8 \mathrm{~Hz}, 1 \mathrm{H}), 8.25$ (dd, $J=8.8,2.7$ $\mathrm{Hz}, 1 \mathrm{H}), 8.11(\mathrm{dd}, J=8.1,1.2 \mathrm{~Hz}, 1 \mathrm{H}), 7.81(\mathrm{dd}, J=7.6,1.6 \mathrm{~Hz}, 1 \mathrm{H}), 7.64(\mathrm{td}, J=7.5,1.2 \mathrm{~Hz}, 1 \mathrm{H}), 7.46$ $(\mathrm{td}, J=7.8,1.6 \mathrm{~Hz}, 1 \mathrm{H}), 4.52(\mathrm{~s}, 2 \mathrm{H}) .{ }^{13} \mathrm{C}-\mathrm{NMR}\left(151 \mathrm{MHz}, \mathrm{DMSO}-d_{6}\right) \delta=149.5,141.4,138.2,135.2$, 133.7, 132.0, 130.7, 129.4, 124.5, 123.4, 122.9, 120.7 (q, $J=322.5 \mathrm{~Hz}), 116.3,45.0 .{ }^{19} \mathrm{~F}-\mathrm{NMR}(565 \mathrm{MHz}$, DMSO- $d_{6}$ ) $\delta=-77.7$. FTIR (ATR, neat) $\tilde{v}=3086,1537,1344,1275,1224,1166,1025,997,818,762$. HRMS (ESI $\left.{ }^{+}, \mathbf{M e O H}\right) \mathrm{m} / z=337.96690[\mathrm{M}-\mathrm{OTf}]^{+}$. Calculated for $\left[\mathrm{C}_{13} \mathrm{H}_{9} \mathrm{INO}_{2}\right]^{+}: \mathrm{m} / z=337.96725 . \mathbf{M p} T=$ $215-220$ (decomp.).

\section{2-Fluoro-10H-dibenzo[b,e]iodinin-5-ium trifluoromethanesulfonate ( $7 \mathrm{~d}$ )}

Following OPP1b, the reaction of (5-fluoro-2-iodophenyl)methanol (5d, $50.4 \mathrm{mg}$ ) with benzene ( $178 \mu \mathrm{L}, 156 \mathrm{mg}, 2.00 \mathrm{mmol}, 10$ eq.) gave after $20 \mathrm{~min}$ for the first reaction step and $16 \mathrm{~h}$ for the second reaction step the product $7 \mathrm{~d}$ ( $67.9 \mathrm{mg}, 0.148 \mathrm{mmol}, 74 \%)$ as a colourless solid.

${ }^{1} \mathrm{H}-N M R\left(601 \mathrm{MHz}\right.$, DMSO- $\left.d_{6}\right) \delta=8.12(\mathrm{dd}, J=8.9,5.3 \mathrm{~Hz}, 1 \mathrm{H}), 8.09$ (dd, $J=$

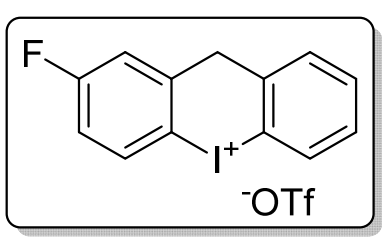
8.1, 1.2 Hz, 1H), 7.77 (dd, $J=7.6,1.6 \mathrm{~Hz}, 1 \mathrm{H}), 7.74(\mathrm{dd}, J=9.3,3.0 \mathrm{~Hz}, 1 \mathrm{H}), 7.62(\mathrm{td}, J=7.5,1.2 \mathrm{~Hz}$, 
1H), 7.44 (ddd, $J=8.2,7.4,1.6 \mathrm{~Hz}, 1 \mathrm{H}$ ), 7.36 (td, $J=8.7,3.0 \mathrm{~Hz}, 1 \mathrm{H}), 4.34(\mathrm{~s}, 2 \mathrm{H}) .{ }^{13} \mathrm{C}-\mathrm{NMR}(151 \mathrm{MHz}$, DMSO- $\left.d_{6}\right) \delta=163.8(\mathrm{~d}, J=249.8 \mathrm{~Hz}), 142.2(\mathrm{~d}, J=8.8 \mathrm{~Hz}), 138.4,135.7(\mathrm{~d}, J=9.0 \mathrm{~Hz}), 133.6,131.8$, $130.6,129.2,120.7$ (q, $J=322.3 \mathrm{~Hz}$ ), 117.6 (d, $J=23.6 \mathrm{~Hz}$ ), 116.3 (d, $J=24.2 \mathrm{~Hz}), 116.2,110.1$ (d, $J=$ $2.4 \mathrm{~Hz}$ ), 45.3. ${ }^{19} \mathrm{~F}-\mathrm{NMR}\left(565 \mathrm{MHz}\right.$, DMSO- $\left.d_{6}\right) \delta=-77.7,-109.3$ (td, $\left.J=8.8,5.3 \mathrm{~Hz}\right)$. FTIR (ATR, neat) $\tilde{v}=$ $3098,1573,1460,1272,1220,1173,1021,998,817,758$. HRMS (ESI ${ }^{+}$, MeOH) $\mathrm{m} / z=310.97245$ [MOTf $]^{+}$. Calculated for $\left[\mathrm{C}_{13} \mathrm{H}_{9} \mathrm{IF}\right]^{+}: 310.97275$. Mp $T=225-230$ (decomp.). The analytical data is in accordance with literature data. ${ }^{[22]}$

\section{2-(Trifluoromethyl)-10H-dibenzo[b,e]iodinin-5-ium trifluoromethanesulfonate (7e)}

Following OPP1b, the reaction of (2-iodo-5-(trifluoromethyl)phenyl)methanol (5e, 60.4 $\mathrm{mg}$ ) with benzene ( $178 \mu \mathrm{L}, 156 \mathrm{mg}, 2.00 \mathrm{mmol}, 10$ eq.) gave after $2 \mathrm{~h}$ for the first reaction step and $16 \mathrm{~h}$ for the second reaction step the product 7 e $(60.1 \mathrm{mg}, 0.118 \mathrm{mmol}, 59 \%)$ as a colourless solid.

Following OPP1C, the reaction of (2-iodo-5(trifluoromethyl)phenyl)methanol (5e, $60.4 \mathrm{mg}$ ) with benzene $(178 \mu \mathrm{L}$, $156 \mathrm{mg}, 2.00 \mathrm{mmol}, 10 \mathrm{eq}$.) gave after $2 \mathrm{~h}$ for the first reaction step and a total of $40 \mathrm{~h}$ for the second reaction step the product $7 \mathrm{e}(67.0 \mathrm{mg}$, $0.131 \mathrm{mmol}, 69 \%$ ) as a colourless solid.

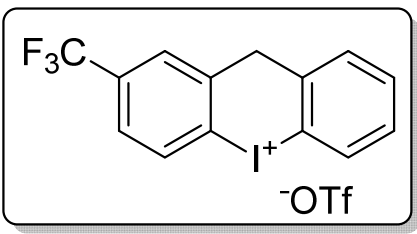

${ }^{1} \mathrm{H}-\mathrm{NMR}\left(601 \mathrm{MHz}\right.$, DMSO- $\left.d_{6}\right) \delta=8.32(\mathrm{~d}, J=8.4 \mathrm{~Hz}, 1 \mathrm{H}), 8.23(\mathrm{~d}, J=2.2 \mathrm{~Hz}, 1 \mathrm{H}), 8.11$ (dd, $J=8.1,1.2$ $\mathrm{Hz}, 1 \mathrm{H}), 7.83(\mathrm{dd}, J=8.6,2.2 \mathrm{~Hz}, 1 \mathrm{H}), 7.79(\mathrm{dd}, J=7.6,1.6 \mathrm{~Hz}, 1 \mathrm{H}), 7.63(\mathrm{td}, J=7.5,1.2 \mathrm{~Hz}, 1 \mathrm{H}), 7.46$ (ddd, $J=8.2,7.4,1.6 \mathrm{~Hz}, 1 \mathrm{H}), 4.45(\mathrm{~s}, 2 \mathrm{H}) .{ }^{13} \mathrm{C}-\mathrm{NMR}\left(151 \mathrm{MHz}, \mathrm{DMSO}-d_{6}\right) \delta=140.7,138.3,134.9,133.7$, $131.9,131.6(q, J=32.6 \mathrm{~Hz}), 130.7,129.3,126.8(\mathrm{q}, J=3.2 \mathrm{~Hz}), 125.5(\mathrm{~d}, J=3.1 \mathrm{~Hz}), 124.4(\mathrm{q}, J=272.9$ $\mathrm{Hz}$ ), $121.8\left(\mathrm{q}, J=322.3 \mathrm{~Hz}\right.$ ), 120.7, 116.1, 45.2. ${ }^{19} \mathrm{~F}-\mathrm{NMR}\left(565 \mathrm{MHz}\right.$, DMSO-d $\left.\boldsymbol{d}_{6}\right) \delta=-61.4,-77.7$. FTIR (ATR, neat) $\tilde{v}=3086,1415,1331,1270,1241,1222,1167,1119,1023,826,762,747$. HRMS (ESI ${ }^{+}$, MeOH) $m / z=360.96921\left[\mathrm{M}-\mathrm{OTf}^{-}\right]^{+}$. Calculated for $\left[\mathrm{C}_{14} \mathrm{H}_{9} \mathrm{~F}_{3} \mathrm{l}^{+}: \mathrm{m} / \mathrm{z}=360.96955\right.$. Mp $T=179-181$.

\section{2-Chloro-10H-dibenzo[b,e]iodinin-5-ium trifluoromethanesulfonate (7f)}

Following OPP1b, the reaction of (5-chloro-2-iodophenyl)methanol (5f, $63.7 \mathrm{mg}$ ) with benzene ( $178 \mu \mathrm{L}, 156 \mathrm{mg}, 2.00 \mathrm{mmol}, 10$ eq.) gave after $15 \mathrm{~min}$ for the first reaction step and $16 \mathrm{~h}$ for the second reaction step the product $7 \mathrm{f}$ ( $55.2 \mathrm{mg}, 0.116 \mathrm{mmol}, 58 \%$ ) as a colourless solid.

${ }^{1} \mathrm{H}-\mathrm{NMR}\left(601 \mathrm{MHz}\right.$, DMSO- $\left.d_{6}\right) \delta=8.09$ (dd, $\left.J=8.1,1.2 \mathrm{~Hz}, 1 \mathrm{H}\right), 8.08(\mathrm{~d}$,

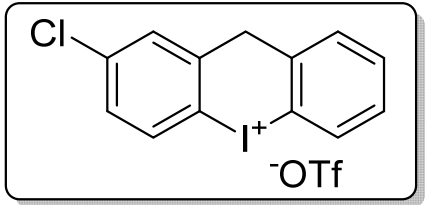
$J=8.6 \mathrm{~Hz}, 1 \mathrm{H}$ ), 7.95 (d, $J=2.5 \mathrm{~Hz}, 1 \mathrm{H}), 7.77$ (dd, $J=7.6,1.6 \mathrm{~Hz}, 1 \mathrm{H}), 7.62$ (td, $J=7.5,1.2 \mathrm{~Hz}, 1 \mathrm{H}$ ), 7.54 (dd, $J=8.6,2.5 \mathrm{~Hz}, 1 \mathrm{H}), 7.44(\mathrm{td}, J=8.1,1.6 \mathrm{~Hz}, 1 \mathrm{H}), 4.33(\mathrm{~s}, 2 \mathrm{H}) .{ }^{13} \mathrm{C}-\mathrm{NMR}\left(151 \mathrm{MHz}\right.$, DMSO-d $\left.d_{6}\right) \delta=$ $141.4,138.4,136.6,135.2,133.6,131.9,130.7,130.1,129.2,128.8,120.7$ (q, $J=322.2 \mathrm{~Hz}$ ), 116.2, 114.1, 45.1. ${ }^{19}$ F-NMR (565 MHz, DMSO-d $\left.d_{6}\right) \delta=-77.7$. FTIR (ATR, neat) $\tilde{v}=3085,1556,1453,1276$, 1220, 1171, 1021, 993, 785, 756. HRMS (ESI $\left.{ }^{+}, \mathrm{MeOH}\right) \mathrm{m} / \mathrm{z}=326.94288$ [M-OTf] ${ }^{+}$. Calculated for $\left[\mathrm{C}_{13} \mathrm{H}_{9} \mathrm{ClI}\right]^{+}: \mathrm{m} / z=326.94320 . \mathrm{Mp} T=216-218$.

\section{2-Cyano-10H-dibenzo[b,e]iodinin-5-ium trifluoromethanesulfonate $(7 \mathrm{~g})$}

Following OPP1c, the reaction of 3-(hydroxymethyl)-4-iodobenzonitrile $(5 \mathrm{~g}, 51.8 \mathrm{mg}$ ) with benzene $(178 \mu \mathrm{L}, 156 \mathrm{mg}, 2.00 \mathrm{mmol}, 10$ eq.) gave after $16 \mathrm{~h}$ for the first reaction step and a total of $48 \mathrm{~h}$ for the second reaction step the product $\mathbf{7 g}(42.6 \mathrm{mg}, 91.2 \mu \mathrm{mol}, 46 \%)$ as a pale yellow solid.

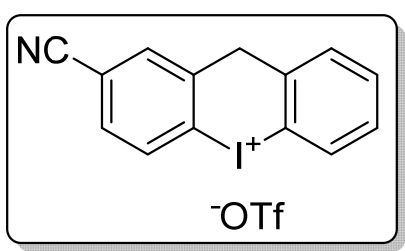

${ }^{1} \mathrm{H}-N M R\left(601 \mathrm{MHz}\right.$, DMSO- $\left.d_{6}\right) \delta=8.30(\mathrm{~d}, J=8.3 \mathrm{~Hz}, 1 \mathrm{H}), 8.29(\mathrm{~d}, J=1.9 \mathrm{~Hz}, 1 \mathrm{H}), 8.10(\mathrm{dd}, J=8.2,1.2$ $\mathrm{Hz}, 1 \mathrm{H}), 7.90(\mathrm{dd}, J=8.3,2.0 \mathrm{~Hz}, 1 \mathrm{H}), 7.79(\mathrm{dd}, J=7.6,1.6 \mathrm{~Hz}, 1 \mathrm{H}), 7.63(\mathrm{td}, J=7.5,1.2 \mathrm{~Hz}, 1 \mathrm{H}), 7.46$ 
(td, $J=7.7,1.6 \mathrm{~Hz}, 1 \mathrm{H}), 4.39$ (s, 2H). ${ }^{13} \mathrm{C}-\mathrm{NMR}\left(151 \mathrm{MHz}\right.$, DMSO-d $\left.d_{6}\right) \delta=140.6,138.1,134.8,133.7$, 133.4, 132.1, 132.0, 130.7, 129.4, 121.6, 120.7 (q, $J=322.6 \mathrm{~Hz}$ ), 117.5, 116.2, 114.3, 45.1. ${ }^{19} \mathrm{~F}-\mathrm{NMR}$ (565 MHz, DMSO- $d_{6}$ ) $\delta=-77.7$. FTIR (ATR, neat) $\tilde{v}=3090,2234,1522,1461,1273,1236,1156,1024$, 758, 741. HRMS (ESI ${ }^{+}$, MeOH) $m / z=317.97716[\mathrm{M}-\mathrm{OTf}]^{+}$. Calculated for $\left[\mathrm{C}_{14} \mathrm{H}_{\mathrm{g}} \mathrm{IN}\right]^{++}: \mathrm{m} / z=317.97742$. Mp $T=165-170$ (decomp).

\section{3-Bromo-10H-dibenzo[b,e]iodinin-5-ium trifluoromethanesulfonate $(7 \mathrm{~h})$}

Following OPP1b, the reaction of (4-bromo-2-iodophenyl)methanol (5h, $62.6 \mathrm{mg}$ ) with benzene ( $178 \mu \mathrm{L}, 156 \mathrm{mg}, 2.00 \mathrm{mmol}, 10$ eq.) gave after $5 \mathrm{~min}$ for the first reaction step and $16 \mathrm{~h}$ for the second reaction step the product $7 \mathrm{~h}(83.9 \mathrm{mg}, 0.161 \mathrm{mmol}, 81 \%)$ as a colourless solid.

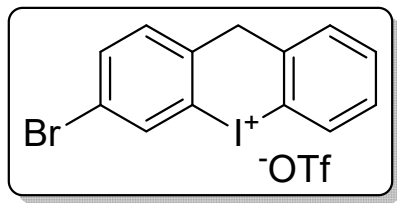

${ }^{1} \mathrm{H}-\mathrm{NMR}\left(601 \mathrm{MHz}\right.$, DMSO- $\left.d_{6}\right) \delta=8.27(\mathrm{~d}, J=2.0 \mathrm{~Hz}, 1 \mathrm{H}), 8.10$ (dd, $\left.J=8.1,1.2 \mathrm{~Hz}, 1 \mathrm{H}\right), 7.83$ (dd, $J=8.1$, $2.0 \mathrm{~Hz}, 1 \mathrm{H}$ ), 7.79 (dd, J = 7.6, $1.6 \mathrm{~Hz}, 1 \mathrm{H}$ ), $7.75(\mathrm{~d}, J=8.2 \mathrm{~Hz}, 1 \mathrm{H}$ ), 7.62 (td, J = 7.5, $1.2 \mathrm{~Hz}, 1 \mathrm{H}), 7.44$ (td, $J=7.7,1.7 \mathrm{~Hz}, 1 \mathrm{H}), 4.30(\mathrm{~s}, 2 \mathrm{H}) .{ }^{13} \mathrm{C}-\mathrm{NMR}\left(151 \mathrm{MHz}, \mathrm{DMSO}-d_{6}\right) \delta=138.7,138.5,135.5,134.5,133.7$, 131.9, 131.8, 130.6, 129.2, 121.75 (q, $J=322 \mathrm{~Hz}$ ), 120.4, 117.0, 116.2, 44.9. ${ }^{19} \mathrm{~F}-\mathrm{NMR}$ (565 MHz, DMSO$\left.\boldsymbol{d}_{6}\right) \delta=-77.7$. FTIR (ATR, neat) $\tilde{v}=3097,1576,1457,1379,1282,1233,1159,1025,842,751$. HRMS (ESI $\left.{ }^{+}, \mathbf{M e O H}\right) m / z=370.89224\left[\mathrm{M}-\mathrm{OTf}^{-}\right]^{+}$. Calculated for $\left[\mathrm{C}_{13} \mathrm{H}_{9} \mathrm{Brl}\right]^{+}: \mathrm{m} / z=370.89269$.

Mp $T=210-215$ (decomp.).

\section{4-Bromo-10H-dibenzo[b,e]iodinin-5-ium trifluoromethanesulfonate (7i)}

Following OPP1b, the reaction of (3-bromo-2-iodophenyl)methanol (5i, $62.6 \mathrm{mg}$ ) with benzene ( $178 \mu \mathrm{L}, 156 \mathrm{mg}, 2.00 \mathrm{mmol}, 10$ eq.) gave after $10 \mathrm{~min}$ for the first reaction step and $16 \mathrm{~h}$ for the second reaction step the product $\mathbf{7 i}$ ( $57.2 \mathrm{mg}, 0.110 \mathrm{mmol}, 55 \%$ ) as a colourless solid.

${ }^{1} \mathrm{H}-\mathrm{NMR}\left(601 \mathrm{MHz}\right.$, DMSO- $\left.\mathbf{d}_{6}\right) \delta=8.17(\mathrm{dd}, J=8.2,1.1 \mathrm{~Hz}, 1 \mathrm{H}), 7.85$ (dd, $J=7.6$,

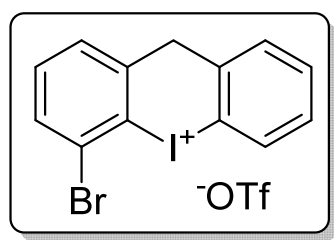
$1.6 \mathrm{~Hz}, 1 \mathrm{H}$ ), 7.82 (dd, $J=7.6,1.3 \mathrm{~Hz}, 1 \mathrm{H}$ ), 7.79 (dd, $J=8.0,1.3 \mathrm{~Hz}, 1 \mathrm{H}), 7.63(\mathrm{td}, J=7.5,1.2 \mathrm{~Hz}, 1 \mathrm{H}), 7.57$ (dd, $J=7.7 \mathrm{~Hz}, 1 \mathrm{H}$ ), 7.45 (ddd, $J=8.3,7.4,1.7 \mathrm{~Hz}, 1 \mathrm{H}), 4.43(\mathrm{~s}, 2 \mathrm{H}) .{ }^{13} \mathrm{C}-\mathrm{NMR}\left(151 \mathrm{MHz}\right.$, DMSO-d $\left.\mathbf{d}_{6}\right) \delta=$ 143.2, 139.1, 134.3, 134.0, 132.5, 132.2, 131.0, 129.8, 129.7, 125.6, 122.0, 121.1 (q, $J=322.3 \mathrm{~Hz}$ ), 118.9, 48.0. ${ }^{19} \mathrm{~F}$-NMR (565 MHz, DMSO-d $\left.d_{6}\right) \delta=-77.7$. FTIR (ATR, neat) $\tilde{v}=3084,1556,1442,1284$, $1233,1218,1161,1021,991,785,755$. HRMS (ESI ${ }^{+}$MeOH) $\mathrm{m} / z=370.89243$ [M-OTf] $]^{+}$. Calculated for $\left[\mathrm{C}_{13} \mathrm{H}_{9} \mathrm{Brl}\right]^{+}: \mathrm{m} / \mathrm{z}=370.89269 . \mathrm{Mp} T=205-210$.

\section{2,4-Dichloro-10H-dibenzo[b,e]iodinin-5-ium trifluoromethanesulfonate $(7 \mathrm{j})$}

Following OPP1b, the reaction of (3,5-dichloro-2-iodophenyl)methanol ( $5 \mathbf{j}, 60.6 \mathrm{mg}$ ) with benzene ( $178 \mu \mathrm{L}, 156 \mathrm{mg}, 2.00 \mathrm{mmol}, 10$ eq.) gave after $4 \mathrm{~h}$ for the first reaction step and a total of $40 \mathrm{~h}$ for the second reaction step the product $7 \mathbf{j}(43.5 \mathrm{mg}, 0.0890 \mathrm{mmol}, 45 \%$ ) as a colourless solid.

${ }^{1} \mathrm{H}-\mathrm{NMR}\left(601 \mathrm{MHz}\right.$, DMSO- $\left.d_{6}\right) \delta=8.15(\mathrm{dd}, J=8.2,1.1 \mathrm{~Hz}, 1 \mathrm{H}), 7.96(\mathrm{~d}, J$

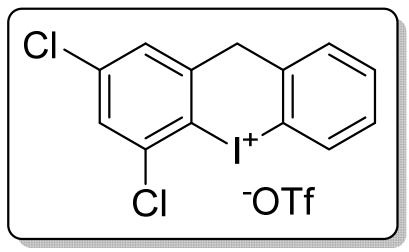
$=2.2 \mathrm{~Hz}, 1 \mathrm{H}), 7.93(\mathrm{~d}, J=2.2 \mathrm{~Hz}, 1 \mathrm{H}), 7.80(\mathrm{dd}, J=7.6,1.6 \mathrm{~Hz}, 1 \mathrm{H}), 7.64(\mathrm{td}, J=7.5,1.2 \mathrm{~Hz}, 1 \mathrm{H}), 7.46$ (td, $J=7.8,1.7 \mathrm{~Hz}, 1 \mathrm{H}), 4.44(\mathrm{~s}, 2 \mathrm{H}) .{ }^{13} \mathrm{C}-\mathrm{NMR}\left(151 \mathrm{MHz}\right.$, DMSO- $\left.d_{6}\right) \delta=144.2,138.2,137.5,136.1,133.8$, 132.1, 130.7, 129.4, 128.8, 128.0, 120.7 (q, $J=322.2 \mathrm{~Hz}$ ), 118.2, 117.0, 46.6. ${ }^{19}$ F-NMR (565 MHz, DMSO$\left.d_{6}\right) \delta=-77.7$. FTIR (ATR, neat) $\tilde{v}=3070,1553,1465,1433,1287,1218,1155,1020,863$, 759. HRMS $\left(\mathbf{E S I}^{+}\right.$, MeOH) $m / z=360.90383\left[\mathrm{M}-\mathrm{OTf}^{-}\right]^{+}$. Calculated for $\left[\mathrm{C}_{13} \mathrm{H}_{8} \mathrm{Cl}_{2} \mathrm{l}^{+}: \mathrm{m} / \mathrm{z}=360.90423\right.$. Mp $T=185.5-$ 187.5. 
Following OPP1a, the reaction of (2-iodophenyl)(phenyl)methanol (5k, $62.0 \mathrm{mg}$ ) with benzene ( $500 \mu \mathrm{L}$, approx. 28 eq.) was completed after $5 \mathrm{~min}$ for the first reaction step and $2 \mathrm{~h}$ for the second reaction step. The combined organic phases were filtered over a small plug of silica which was washed with $\mathrm{DCM} / \mathrm{MeOH}$ 25:1 $10 \mathrm{~mL}$ ). After removal of the solvents under reduced pressure and purification as described in OPP1a, the product $\mathbf{7 k}(82.0 \mathrm{mg}$,

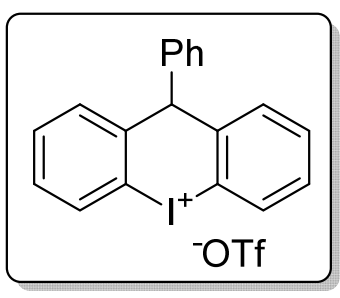
$0.158 \mathrm{mmol}, 79 \%$ ) was obtained as a colourless solid.

${ }^{1} \mathrm{H}-\mathrm{NMR}\left(601 \mathrm{MHz}\right.$, DMSO- $\left.d_{6}\right) \delta=8.13(\mathrm{dd}, J=8.1,1.2 \mathrm{~Hz}, 2 \mathrm{H}), 8.02(\mathrm{dd}, J=7.6,1.6 \mathrm{~Hz}, 2 \mathrm{H}), 7.76$ (td, $J=7.5,1.2 \mathrm{~Hz}, 2 \mathrm{H}), 7.53(\mathrm{td}, J=7.7,1.6 \mathrm{~Hz}, 2 \mathrm{H}), 7.34-7.25(\mathrm{~m}, 3 \mathrm{H}), 6.80-6.77(\mathrm{~m}, 2 \mathrm{H}), 6.22(\mathrm{~s}, 1 \mathrm{H})$.

${ }^{13} \mathrm{C}-\mathrm{NMR}\left(151 \mathrm{MHz}\right.$, DMSO- $\left.d_{6}\right) \delta=140.1,138.0,134.6,132.9,132.0,129.7,128.7,127.7,127.3,120.7$ (q, $J=322.2 \mathrm{~Hz}$ ), 115.2, 57.6. ${ }^{19} \mathrm{~F}-\mathrm{NMR}\left(565 \mathrm{MHz}\right.$, DMSO-d $\left.d_{6}\right) \delta=-77.7$. FTIR (ATR, neat) $\tilde{v}=3086$, 1493, 1461, 1440, 1245, 1156, 1025, 164, 758, 731. HRMS (ESI ${ }^{+}$, MeOH) $m / z=369.01317$ [M-OTf] ${ }^{+}$. Calculated for $\left[\mathrm{C}_{19} \mathrm{H}_{14}\right]^{+}: \mathrm{m} / z=369.01347$. $\mathrm{Mp} T=215-218$ (decomp.). The analytical data is in accordance with literature data. ${ }^{[22]}$

10-(3,5-Bis(trifluoromethyl)phenyl)-10H-dibenzo[b,e]iodinin-5-ium trifluoromethanesulfonate (7I)

Following OPP1a, the reaction of (3,5-(bis(trifluoromethyl)phenyl)(2iodophenyl)methanol (5I, $89.2 \mathrm{mg}$ ) with benzene (178 $\mu \mathrm{L}, 156 \mathrm{mg}, 2.00 \mathrm{mmol}$, 10.0 eq.) gave after $5 \mathrm{~min}$ for the first reaction step and $16 \mathrm{~h}$ for the second reaction step the product $7 \mathrm{l}(95.4 \mathrm{mg}, 0.146 \mathrm{mmol}, 73 \%)$ as a colourless solid.

${ }^{1} \mathrm{H}-\mathrm{NMR}\left(601 \mathrm{MHz}\right.$, DMSO- $\left.d_{6}\right) \delta=8.19(\mathrm{dd}, J=8.1,1.2 \mathrm{~Hz}, 2 \mathrm{H}), 8.17(\mathrm{~s}, 1 \mathrm{H})$, 8.11 (dd, $J=7.7,1.6 \mathrm{~Hz}, 2 \mathrm{H}), 7.82(\mathrm{td}, J=7.5,1.2 \mathrm{~Hz}, 2 \mathrm{H}), 7.60$ (td, $J=7.8,1.6$ $\mathrm{Hz}, 2 \mathrm{H}), 7.36(\mathrm{~s}, 2 \mathrm{H}), 6.45(\mathrm{~s}, 1 \mathrm{H}) .{ }^{13} \mathrm{C}-\mathrm{NMR}(151 \mathrm{MHz}$, DMSO-d $\left.)\right) \delta=141.6$,

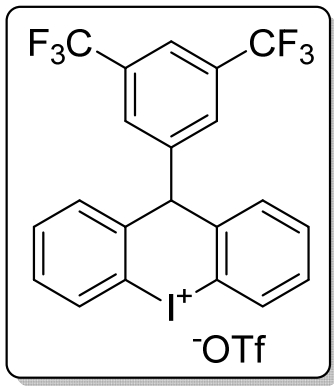
$138.5,135.0,133.1,132.5,130.8(q, J=32.9 \mathrm{~Hz}), 130.4,128.0-127.8(\mathrm{~m}), 122.9(\mathrm{q}, J=273.1 \mathrm{~Hz}), 122.0$ - $121.6(\mathrm{~m}), 120.7$ (q, $J=322.3 \mathrm{~Hz}), 115.2,56.9 .{ }^{19} \mathrm{~F}-\mathrm{NMR}\left(565 \mathrm{MHz}\right.$, DMSO- $\left.d_{6}\right) \delta=-61.4,-77.8$. FTIR (ATR, neat) $\tilde{v}=3068,1487,1454,1272,1235,1221,1163,1025,805,756,737$. HRMS (ESI $^{+}$, MeOH) $m / z=504.98785\left[\mathrm{M}-\text { OTf }^{-}\right]^{+}$. Calculated for $\left[\mathrm{C}_{21} \mathrm{H}_{12} \mathrm{~F}_{6}\right]^{+}: \mathrm{m} / z=504.98824 . \mathrm{Mp} T=188-190$.

\section{2-Chloro-10-(2-fluorophenyl)-10H-dibenzo[b,e]iodinin-5-ium trifluoromethanesulfonate $(7 \mathrm{~m})$}

Following OPP1a, the reaction of (5-chloro-2-iodophenyl)(2fluorophenyl)methanol $(5 \mathrm{~m}, 72.5 \mathrm{mg})$ with benzene $(178 \mu \mathrm{L}, 156 \mathrm{mg}$, $2.00 \mathrm{mmol}, 10.0$ eq.) gave after $5 \mathrm{~min}$ for the first reaction step and $16 \mathrm{~h}$ for the second reaction step the product $7 \mathrm{~m}(67.1 \mathrm{mg}, 0.118 \mathrm{mmol}, 59 \%)$ as a pale yellow solid.

${ }^{1} \mathrm{H}-\mathrm{NMR}\left(601 \mathrm{MHz}\right.$, DMSO- $\left.\boldsymbol{d}_{6}\right) \delta=8.15-8.06(\mathrm{~m}, 3 \mathrm{H}), 7.94(\mathrm{dd}, J=7.8$, $1.9 \mathrm{~Hz}, 1 \mathrm{H}), 7.73(\mathrm{td}, J=7.5,1.3 \mathrm{~Hz}, 1 \mathrm{H}), 7.63(\mathrm{dd}, J=8.6,2.5 \mathrm{~Hz}, 1 \mathrm{H}), 7.52$

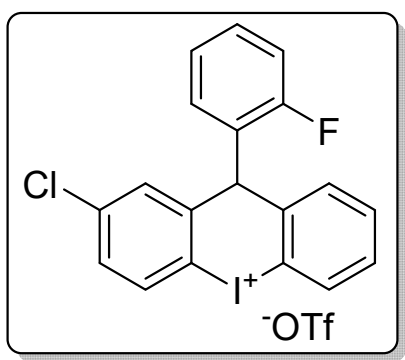
(td, $J=7.7,1.6 \mathrm{~Hz}, 1 \mathrm{H}), 7.42$ (tdd, $J=7.5,5.0,1.8 \mathrm{~Hz}, 1 \mathrm{H}), 7.23$ (ddd, $J=11.6,8.3,1.3 \mathrm{~Hz}, 1 \mathrm{H}$ ), 7.16 (td, $J=7.7,1.3 \mathrm{~Hz}, 1 \mathrm{H}), 6.85-6.78(\mathrm{~m}, 1 \mathrm{H}), 6.38(\mathrm{~s}, 1 \mathrm{H}) .{ }^{13} \mathrm{C}-\mathrm{NMR}\left(151 \mathrm{MHz}\right.$, DMSO-d $\left.d_{6}\right) \delta=160.9(\mathrm{~d}, J=$ $248.0 \mathrm{~Hz}$ ), 141.3, 138.4, 136.8, 136.0, 134.5, $132.9(\mathrm{~d}, J=2.7 \mathrm{~Hz}), 132.2(\mathrm{~d}, J=3.0 \mathrm{~Hz}), 132.0,130.5(\mathrm{~d}$, $J=8.5 \mathrm{~Hz}$ ), 129.9 (d, $J=3.5 \mathrm{~Hz}), 129.9,129.5,124.4(\mathrm{~d}, J=3.3 \mathrm{~Hz}), 123.2(\mathrm{~d}, J=12.2 \mathrm{~Hz}), 120.7(\mathrm{q}, J=$ $322.2 \mathrm{~Hz}$ ), $116.8(\mathrm{~d}, J=21.5 \mathrm{~Hz}), 115.8,113.7,53.2 .{ }^{19} \mathrm{~F}-\mathrm{NMR}\left(565 \mathrm{MHz}\right.$, DMSO- $d_{6}$ ) $\delta=-77.7,-109.4$. 
FTIR (ATR, neat) $\tilde{v}=3068,1487,1454,1272,1235,1221,1163,1025,805,756,737$. HRMS (ESI $^{+}$, MeOH) $m / z=420.96470[\mathrm{M}-\mathrm{OTf}]^{+}$. Calculated for $\left[\mathrm{C}_{19} \mathrm{H}_{12} \mathrm{ClFI}\right]^{+}: \mathrm{m} / z=420.96508$. Mp T $=205-210$ (decomp.).

\section{1,4-Dimethyl-10H-dibenzo[b,e]iodinin-5-ium trifluoromethanesulfonate $(7 \mathrm{n})$}

Following OPP1a, the reaction of $o$-iodobenzylalcohol $(5 \mathrm{a}, 46.8 \mathrm{mg})$ with $p$ xylene ( $123 \mu \mathrm{L}, 106 \mathrm{mg}, 1.00 \mathrm{mmol}, 5.00$ eq.) gave after $5 \mathrm{~min}$ for the first reaction step and $2 \mathrm{~h}$ for the second reaction step the product $7 \mathrm{n}(87.3 \mathrm{mg}$, $0.186 \mathrm{mmol}, 93 \%$ ) as a colourless solid.

${ }^{1} \mathrm{H}-\mathrm{NMR}\left(601 \mathrm{MHz}, \mathrm{DMSO}-\mathrm{d}_{6}\right) \delta=8.10(\mathrm{dd}, J=8.1,1.2 \mathrm{~Hz}, 1 \mathrm{H}), 7.94(\mathrm{dd}, J=7.6$,

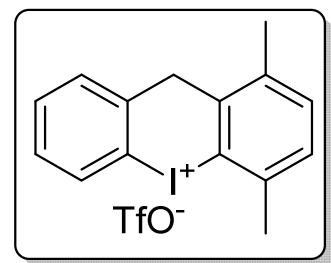
$1.6 \mathrm{~Hz}, 1 \mathrm{H}), 7.61(\mathrm{td}, J=7.5,1.2 \mathrm{~Hz}, 1 \mathrm{H}), 7.42(\mathrm{td}, J=8.4,7.8,1.6 \mathrm{~Hz}, 1 \mathrm{H}), 7.38$ (d, J = 7.7 Hz, 1H), $7.27(\mathrm{~d}, J=7.6 \mathrm{~Hz}, 1 \mathrm{H}), 4.31(\mathrm{~s}, 2 \mathrm{H}), 2.59(\mathrm{~s}, 3 \mathrm{H}), 2.56(\mathrm{~s}, 3 \mathrm{H})$.

${ }^{13} \mathrm{C}-\mathrm{NMR}\left(151 \mathrm{MHz}, \mathrm{DMSO}-d_{6}\right) \delta=139.1,138.0,137.4,135.7,133.6,133.1,131.8,130.5,128.9,128.9$, $122.1,120.7$ (q, $J=322.6 \mathrm{~Hz}$ ), 117.6, 44.0, 24.6, 20.0. ${ }^{19} \mathrm{~F}-\mathrm{NMR}$ (565 MHz, DMSO- $d_{6}$ ) $\delta=-77.7$. FTIR (ATR, neat) $\tilde{v}=3095,3015,1457,1441,1243,1222,1154,818,770,753$. HRMS (ESI $^{+}$, MeOH) $\mathrm{m} / \mathrm{z}=$ $321.01322[\mathrm{M}-\mathrm{OTf}]^{+}$. Calculated for $\left[\mathrm{C}_{15} \mathrm{H}_{14} \mathrm{l}^{+}: \mathrm{m} / \mathrm{z}=321.01347\right.$. Mp T $=207-210$ (decomp.).

\subsection{General Procedures for the One-Pot Reaction Utilizing Secondary Aliphatic Benzyl Alcohols (OPP2)}

A solution of the secondary $o$-iodobenzyl alcohol $(5,200 \mu \mathrm{mol}, 1$ eq.) and the arene $(1.00 \mathrm{mmol}, 5 \mathrm{eq}$.) in DCM (1 mL) was cooled to $0{ }^{\circ} \mathrm{C}$ and $\mathrm{HBF}_{4}\left(29.5 \mu \mathrm{L}, 35.1 \mathrm{mg}, 200 \mu \mathrm{mol}, 1\right.$ eq., $\left.50 \% \mathrm{w} / \mathrm{w}_{\text {in Et }} \mathrm{O}\right)$ was added. The solution was stirred until complete conversion of the alcohol as observed via TLC and then DCM $(1 \mathrm{~mL})$ and $m C P B A(98.6 \mathrm{mg}, 400 \mu \mathrm{mol}, 2$ eq., $70 \% \mathrm{w} / \mathrm{w}$ ) were added and stirred for $10 \mathrm{~min}$. Further $\mathrm{HBF}_{4}\left(59.0 \mu \mathrm{L}, 70.3 \mathrm{mg}, 400 \mu \mathrm{mol}, 1\right.$ eq., $50 \% \mathrm{w} / \mathrm{w}$ in $\left.\mathrm{Et}_{2} \mathrm{O}\right)$ was added and stirred until complete conversion of the intermediate. $\mathrm{H}_{2} \mathrm{O}(2 \mathrm{~mL})$ was added, the phases separated and the aqueous phase was extracted with DCM $(5 \times 2 \mathrm{~mL})$. The solvent of the combined organic phases was removed under reduced pressure. The residue was added to a small pad of silica, washed with DCM $(120 \mathrm{~mL})$ and afterwards eluted with EtOAc $(80 \mathrm{~mL})$. The ethyl acetate phase was evaporated to a minimum amout and the product precipitated by addition of $\mathrm{Et}_{2} \mathrm{O}$.

\subsection{Substrates Derived from Secondary Aliphatic Benzyl Alcohols}

\section{3,10-Dimethyl-10H-dibenzo[b,e]iodinin-5-ium tetrafluoroborate (8a)}

Following OPP2, 1-(2-iodophenyl)ethan-1-ol (5n, $49.6 \mathrm{mg})$ and toluene (106 $\mu$ l, $92.1 \mathrm{mg}$ ) gave after $10 \mathrm{~min}$ for the first reaction step and $2 \mathrm{~h}$ for the second reaction step the product $8 \mathrm{a}(68.0 \mathrm{mg}, 167 \mu \mathrm{mol}, 83 \%)$ as a slightly yellow solid.

\section{Upscaled reaction:}

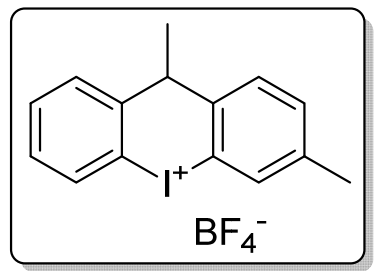

A solution of the 1(2-iodophenyl)ethan-1-ol ( $5 \mathrm{n}, 1.24 \mathrm{~g}, 5.00 \mathrm{mmol}, 1$ eq.) and toluene ( $2.65 \mathrm{~mL}, 2.30 \mathrm{~g}$, $25.0 \mathrm{mmol}, 5$ eq.) in DCM ( $25 \mathrm{~mL}$ ) was cooled to $0{ }^{\circ} \mathrm{C}$ and $\mathrm{HBF}_{4}(0.74 \mathrm{~mL}, 878 \mathrm{mg}, 200 \mu \mathrm{mol}, 1$ eq., $50 \%$ $\mathrm{w} / \mathrm{w}$ in $\mathrm{Et}_{2} \mathrm{O}$ ) was added in four portions over $5 \mathrm{~min}$. The solution was stirred for $10 \mathrm{~min}$ and then DCM $(25 \mathrm{~mL})$ and $m$ CPBA $(2.47 \mathrm{~g}, 10 \mathrm{mmol}, 2$ eq., $70 \% \mathrm{w} / \mathrm{w})$ were added and stirred for $10 \mathrm{~min}$. Further $\mathrm{HBF}_{4}$ $\left(1.48 \mathrm{~mL}, 1.76 \mathrm{~g}, 10.0 \mathrm{mmol}, 2\right.$ eq., $50 \% \mathrm{w} / \mathrm{w}$ in $\left.\mathrm{Et}_{2} \mathrm{O}\right)$ was slowly added and stirred at $0{ }^{\circ} \mathrm{C}$ for $3.5 \mathrm{~h}$ 
until complete conversion of the intermediate. $\mathrm{H}_{2} \mathrm{O}(50 \mathrm{~mL})$ was added, the phases were separated and the aqueous phase was extracted with DCM $(5 \times 20 \mathrm{~mL})$. The combined organic phases were dried over $\mathrm{Na}_{2} \mathrm{SO}_{4}$ and concentrated under reduced pressure. The residue was added to a pad of silica, washed with DCM $(600 \mathrm{~mL})$ and afterwards eluted with EtOAc $(300 \mathrm{~mL})$. The ethyl acetate phase was evaporated to a minimum amount and the product precipitated by addition of $\mathrm{Et}_{2} \mathrm{O}$ to obtain the product in approx. $90 \%$ purity. After refluxing in EtOAc and slow cooling to $4{ }^{\circ} \mathrm{C}$, the pure product $8 \mathrm{a}$ was obtained as a colourless solid (717 mg, $1.76 \mathrm{mmol}, 35 \%$ ).

${ }^{1} \mathrm{H}-\mathrm{NMR}$ (DMSO- $\left.\boldsymbol{d}_{6}, 601 \mathrm{MHz}\right) \delta=8.11(\mathrm{dd}, J=8.0,0.8 \mathrm{~Hz}, 1 \mathrm{H}), 7.93(\mathrm{~s}, 1 \mathrm{H}), 7.73(\mathrm{~d}, J=6.9 \mathrm{~Hz}, 1 \mathrm{H}$ ), $7.63(\mathrm{td}, J=8.4,7.6,0.9 \mathrm{~Hz}, 1 \mathrm{H}), 7.62(\mathrm{~d}, J=7.9 \mathrm{~Hz}, 1 \mathrm{H}), 7.45(\mathrm{~d}, J=7.2 \mathrm{~Hz}, 1 \mathrm{H}), 7.42(\mathrm{td}, J=8.0,1.4 \mathrm{~Hz}$, 1H), 4.21 (bs, 1H), 2.36 (s, 3H) 1.72 (bs, 3H). ${ }^{13} \mathrm{C}-\mathrm{NMR}$ (DMSO-d $\left.\mathbf{d}_{6}, 151 \mathrm{MHz}\right) \delta=141.8,138.8,138.6$, 133.9, 133.8, 132.4, 131.8, 129.0, 128.7, 128.4, 115.5 (2 C), 47.3, 20.2, 16.3. ${ }^{19} \mathrm{~F}-\mathrm{NMR}$ (DMSO-d $d_{6}$, $565 \mathrm{MHz}) \delta=-148.3$. FTIR (ATR, neat) $\tilde{v}=3096,2976,2926,1458,1438,1386,1015,831,769,745$.

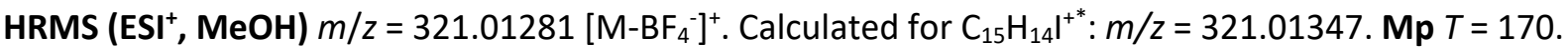

\section{0-Ethyl-3-methyl-10H-dibenzo[b,e]iodinin-5-ium tetrafluoroborate $(8 \mathrm{~b})$}

Following OPP2, 1-(2-iodophenyl)propan-1-ol (5o, $52.4 \mathrm{mg})$ and toluene $(106 \mu \mathrm{l}, 92.1 \mathrm{mg}$ ) gave after $10 \mathrm{~min}$ for the first reaction step and $2 \mathrm{~h}$ for the second reaction step the product $8 \mathbf{b}(49.8 \mathrm{mg}, 118 \mu \mathrm{mol}, 59 \%)$ as a colourless solid.

${ }^{1} \mathrm{H}-\mathrm{NMR}$ (DMSO- $\left.d_{6}, 601 \mathrm{MHz}\right) \delta=8.11(\mathrm{dd}, J=7.9,0.9 \mathrm{~Hz}, 1 \mathrm{H}), 7.92(\mathrm{~s}, 1 \mathrm{H})$,

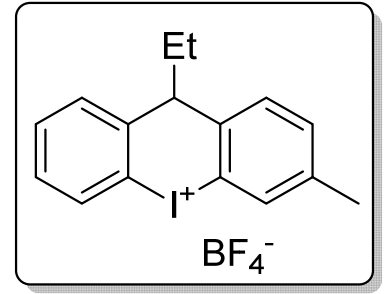

$7.72(\mathrm{dd}, J=7.7,1.6 \mathrm{~Hz}, 1 \mathrm{H}), 7.62(\mathrm{td}, J=7.4,1.2 \mathrm{~Hz}, 1 \mathrm{H}), 7.61(\mathrm{~d}, J=7.7 \mathrm{~Hz}, 1 \mathrm{H}), 7.47-7.42(\mathrm{~m}, 2 \mathrm{H})$, 4.37 (bs, $1 \mathrm{H}), 2.36$ (s, 3H), 1.86 (bs, 2H), 0.88 (bs, 3H). ${ }^{13} \mathrm{C}-\mathrm{NMR}$ (DMSO-d $\left.\boldsymbol{d}_{6}, 151 \mathrm{MHz}\right) \delta=140.4,139.2$, $137.1,134.0,133.9,132.3,131.7,129.3,111.5$ (2 C), 54.8, 25.0, 20.3, 12.2. Due to conformational broadening not all signals were obtained. ${ }^{19} \mathrm{~F}$-NMR (DMSO- $\left.\boldsymbol{d}_{6}, 565 \mathrm{MHz}\right) \boldsymbol{\delta}=-148.3$.. FTIR (ATR, neat) $\tilde{v}=2973,2934,2881,2858,1489,1460,1020,984,827,760$. HRMS $\left._{(E S I}{ }^{+}, \mathrm{MeOH}\right) \mathrm{m} / \mathrm{z}=335.02878$ $\left[\mathrm{M}-\mathrm{BF}_{4}^{-}\right]^{+}$. Calculated for $\mathrm{C}_{16} \mathrm{H}_{16} \mathrm{l}^{+}: \mathrm{m} / \mathrm{z}=335.02912 . \mathrm{Mp} T=205$.

\section{0-Isopropyl-3-methyl-10H-dibenzo[b,e]iodinin-5-ium tetrafluoroborate (8c)}

Following OPP2, 1-(2-iodophenyl)-2-methylpropan-1-ol (5p, $55.2 \mathrm{mg}$ ) and toluene (106 $\mu \mathrm{l}, 92.1 \mathrm{mg})$ gave after $10 \mathrm{~min}$ for the first reaction step and $2 \mathrm{~h}$ for the second reaction step the product $8 \mathrm{c}(38.6 \mathrm{mg}, 88.5 \mu \mathrm{mol}, 44 \%)$ as a colourless solid.

${ }^{1} \mathrm{H}-\mathrm{NMR}$ (DMSO-d $\left.\mathrm{d}_{6}, 601 \mathrm{MHz}\right) \delta=8.11(\mathrm{dd}, J=8.1,1.1 \mathrm{~Hz}, 1 \mathrm{H}), 7.92(\mathrm{~d}, J=$ $1.1 \mathrm{~Hz}, 1 \mathrm{H}$ ), 7.68 (dd, $J=7.7,1.7 \mathrm{~Hz}, 1 \mathrm{H}), 7.60(\mathrm{td}, J=7.5,1.2 \mathrm{~Hz}, 1 \mathrm{H}), 7.57$ (d, $J=7.8 \mathrm{~Hz}, 1 \mathrm{H}), 7.48-7.40(\mathrm{~m}, 2 \mathrm{H}), 4.14(\mathrm{~d}, J=11.0 \mathrm{~Hz}, 1 \mathrm{H}), 2.37(\mathrm{~s}, 3 \mathrm{H})$, $2.31-2.20(\mathrm{~m}, 1 \mathrm{H}), 0.83(\mathrm{~d}, J=5.6 \mathrm{~Hz}, 3 \mathrm{H}), 0.82(\mathrm{~d}, J=5.6 \mathrm{~Hz}, 3 \mathrm{H}) .{ }^{13} \mathrm{C}-\mathrm{NMR}$

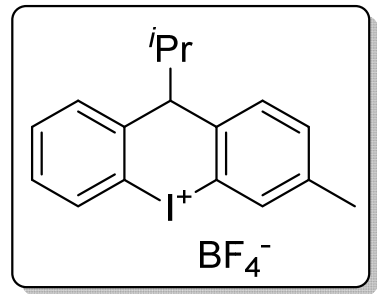
(DMSO-d $\left.d_{6}, 151 \mathrm{MHz}\right) \delta=140.1,139.2,136.9,134.1,134.0,133.1,132.7,132.0,131.3,129.4,111.9$, 111.8, 60.8, 28.3, 21.2, 21.2, 20.3. ${ }^{19} \mathrm{~F}$-NMR (DMSO- $\boldsymbol{d}_{6}, 565 \mathrm{MHz}$ ) $\delta=-148.3$. FTIR (ATR, neat) $\tilde{v}=2971$, 2933, 2906, 2877, 1607, 1463, 1024, 988, 823, 761. HRMS (ESI ${ }^{+}$, MeOH) $m / z=349.04439\left[{\left.\mathrm{M}-\mathrm{BF}_{4}\right]^{+}}^{+}\right.$. Calculated for $\left.\mathrm{C}_{17} \mathrm{H}_{18}\right|^{+}: \mathrm{m} / z=349.04477$. $\mathbf{M p ~ T}=250$ (decomp.). 
Following OPP2, (2-iodophenyl)(phenyl)methanol (5k,62.0 mg) and toluene $(106 \mu \mathrm{l}, 92.1 \mathrm{mg}$ ) gave after $30 \mathrm{~min}$ for the first reaction step and $2 \mathrm{~h}$ for the second reaction step the product $\mathbf{8 d}(34.5 \mathrm{mg}, 73.4 \mu \mathrm{mol}, 37 \%)$ as a colourless solid.

${ }^{1} \mathrm{H}-\mathrm{NMR}\left(601 \mathrm{MHz}\right.$, DMSO- $\left.d_{6}\right) \delta=8.12(\mathrm{dd}, J=8.1,1.2 \mathrm{~Hz}, 1 \mathrm{H}), 8.00(\mathrm{dd}, J=$ 7.6, 1.6 Hz, $1 \mathrm{H}), 7.95-7.92(\mathrm{~m}, 1 \mathrm{H}), 7.89(\mathrm{~d}, J=7.7 \mathrm{~Hz}, 1 \mathrm{H}), 7.75(\mathrm{td}, J=7.5$,

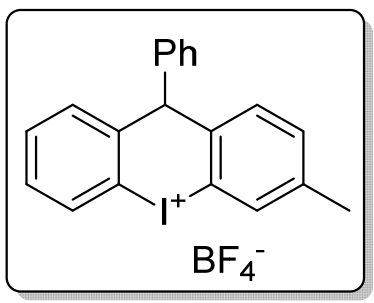
$1.2 \mathrm{~Hz}, 1 \mathrm{H}), 7.57(\mathrm{dd}, J=7.7,0.9 \mathrm{~Hz}, 1 \mathrm{H}), 7.52(\mathrm{td}, J=8.0,1.6 \mathrm{~Hz}, 1 \mathrm{H}), 7.32-7.26(\mathrm{~m}, 3 \mathrm{H}), 6.79(\mathrm{~d}, J=$ $7.9 \mathrm{~Hz}, 2 \mathrm{H}), 6.16(\mathrm{~s}, 1 \mathrm{H}), 2.41(\mathrm{~s}, 3 \mathrm{H}) .{ }^{13} \mathrm{C}-\mathrm{NMR}(151 \mathrm{MHz}$, DMSO-d 6 ) $\delta=140.4,139.6,138.2,137.1$, 134.6, 134.6, 132.8, 132.6, 132.5, 132.0, 129.6, 128.7, 127.7, 127.2, 115.1, 115.1, 57.2, 20.4. ${ }^{19} \mathrm{~F}-\mathrm{NMR}$ (565 MHz, DMSO-d ${ }_{6}$ ) $\delta=-148.3 .{ }^{1} \mathrm{H}-\mathrm{NMR}\left(\mathrm{CD}_{3} \mathrm{OD}, 601 \mathrm{MHz}\right) \delta=8.10(\mathrm{dd}, J=8.1,1.2 \mathrm{~Hz}, 1 \mathrm{H}), 8.01$ (dd, $J=7.6,1.6 \mathrm{~Hz}, 1 \mathrm{H}), 7.93(\mathrm{~s}, 1 \mathrm{H}), 7.88(\mathrm{~d}, J=7.7 \mathrm{~Hz}, 1 \mathrm{H}), 7.77(\mathrm{td}, J=7.5,1.2 \mathrm{~Hz}, 1 \mathrm{H}), 7.59$ (dd, $J=7.7$, $0.8 \mathrm{~Hz}, 1 \mathrm{H}), 7.51(\mathrm{td}, J=7.8,1.6 \mathrm{~Hz}, 1 \mathrm{H}), 7.29-7.22(\mathrm{~m}, 3 \mathrm{H}), 6.84(\mathrm{dt}, J=7.9,1.3 \mathrm{~Hz}, 2 \mathrm{H}), 6.02(\mathrm{~s}, 1 \mathrm{H})$, $2.47(\mathrm{~s}, 3 \mathrm{H}) .{ }^{13} \mathrm{C}-\mathrm{NMR}\left(\mathrm{CD}_{3} \mathrm{OD}, 151 \mathrm{MHz}\right) \delta=142.1,142.1,139.3,138.7,135.5,135.5,134.4,134.2$, 134.0, 133.5, 131.1, 129.8, 129.1, 128.6, 115.2, 115.1, 60.1, 20.9. ${ }^{19} \mathrm{~F}-\mathrm{NMR}$ (CD $\left.{ }_{3} \mathrm{OD}, 565 \mathrm{MHz}\right) \delta=-$ 154.8. FTIR (ATR, neat) $\tilde{v}=3028,1601,1488,1463,1287,1020,835,822,760,730$. HRMS (ESI ${ }^{+}$,

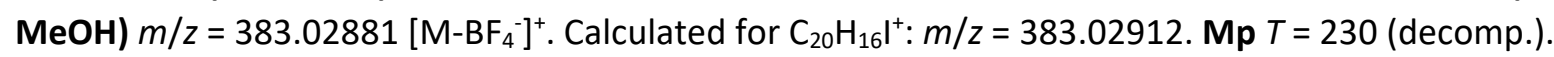

\section{2-Bromo-7,10-dimethyl-10H-dibenzo[b,e]iodinin-5-ium tetrafluoroborate (8e)}

Following OPP2, 1-(5-bromo-2-iodophenyl)ethan-1-ol (5q, $65.4 \mathrm{mg})$ and toluene $(106 \mu \mathrm{l}, 92.1 \mathrm{mg})$ gave after $2 \mathrm{~h}$ for the first reaction step and $4 \mathrm{~h}$ for the second reaction step the product $8 \mathrm{e}(16.9 \mathrm{mg}, 40.1 \mu \mathrm{mol}, 20 \%)$ as a colourless solid.

${ }^{1} \mathrm{H}-\mathrm{NMR}\left(601 \mathrm{MHz}\right.$, DMSO- $\left.d_{6}\right) \delta=8.03(\mathrm{~d}, J=8.5 \mathrm{~Hz}, 1 \mathrm{H}), 7.92(\mathrm{dd}, J=$ 1.6, 0.7 Hz, 1H), $7.90(\mathrm{~s}, 1 \mathrm{H}), 7.66(\mathrm{dd}, J=8.5,2.3 \mathrm{~Hz}, 1 \mathrm{H}), 7.59(\mathrm{~d}, J=$ $7.9 \mathrm{~Hz}, 1 \mathrm{H}), 7.46(\mathrm{ddd}, J=7.9,1.7,0.8 \mathrm{~Hz}, 1 \mathrm{H}), 4.24(\mathrm{~s}, 1 \mathrm{H}), 2.36(\mathrm{~s}$, $3 \mathrm{H}), 1.72(\mathrm{~s}, 3 \mathrm{H}) .{ }^{13} \mathrm{C}-\mathrm{NMR}\left(\mathbf{1 5 1} \mathrm{MHz}, \mathrm{DMSO}-\boldsymbol{d}_{6}\right) \delta=144.5,139.0$,

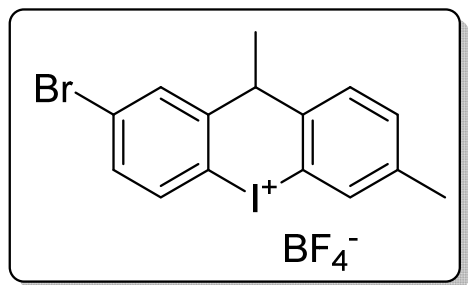
138.1, 135.6, 133.8, 132.5, 131.7, 125.7, 47.0, 20.2. ${ }^{19} \mathrm{~F}-\mathrm{NMR}\left(565 \mathrm{MHz}, \mathrm{DMSO}-\boldsymbol{d}_{6}\right) \delta=-148.3 .{ }^{1} \mathrm{H}-\mathrm{NMR}$ $\left(\mathrm{CD}_{3} \mathrm{OD}, 601 \mathrm{MHz}\right) \delta=7.98(\mathrm{~d}, J=8.5 \mathrm{~Hz}, 1 \mathrm{H}), 7.95(\mathrm{~d}, J=2.3 \mathrm{~Hz}, 1 \mathrm{H}), 7.91(\mathrm{~s}, 1 \mathrm{H}), 7.65(\mathrm{~d}, J=7.9 \mathrm{~Hz}$, $1 \mathrm{H}), 7.61(\mathrm{dd}, J=8.5,2.3 \mathrm{~Hz}, 1 \mathrm{H}), 7.48(\mathrm{~d}, J=7.8 \mathrm{~Hz}, 1 \mathrm{H}), 4.25(\mathrm{bs}, 1 \mathrm{H}), 2.42(\mathrm{~s}, 3 \mathrm{H}), 1.78(\mathrm{bs}, 3 \mathrm{H}) .{ }^{13} \mathrm{C}-$ NMR (CD $\left.{ }_{3} \mathrm{OD}, 151 \mathrm{MHz}\right) \delta=136.2,132.3,129.9,126.6,125.3,124.8,124.0,120.9,118.6,11.3,7.6$. Due to conformational broadening not all signals were obtained. ${ }^{19} \mathrm{~F}-\mathrm{NMR}\left(\mathrm{CD}_{3} \mathrm{OD}, 565 \mathrm{MHz}\right) \delta$ $=-155.0$. FTIR (ATR, neat) $\tilde{v}=3107,2978,1546,1484,1393,1021,875,823,765$. HRMS (ESI ${ }^{+}$, MeOH)

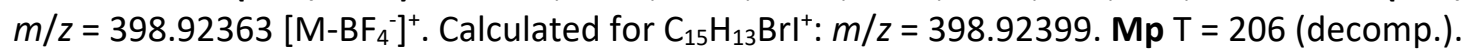

\section{2,3,10-Trimethyl-10H-dibenzo[b,e]iodinin-5-ium (8f)}

Following OPP2, 1-(2-iodophenyl)ethan-1-ol $(5 \mathrm{n}, 49.6 \mathrm{mg})$ and toluene $(121 \mu \mathrm{l}, 106 \mathrm{mg})$ gave after $10 \mathrm{~min}$ for the first reaction step and $2 \mathrm{~h}$ for the second reaction step the product $8 f(47.5 \mathrm{mg}, 0.113 \mu \mathrm{mol}, 56 \%)$ as a colourless solid.

${ }^{1} \mathrm{H}-\mathrm{NMR}\left(601 \mathrm{MHz}, \mathrm{DMSO}-\mathrm{d}_{6}\right) \delta=8.10(\mathrm{dd}, J=8.1,1.2 \mathrm{~Hz}, 1 \mathrm{H}), 7.85(\mathrm{~s}, 1 \mathrm{H})$,

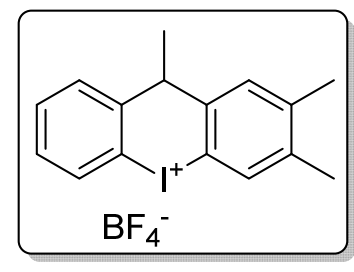
$7.72(\mathrm{dd}, J=7.9,1.6 \mathrm{~Hz}, 1 \mathrm{H}), 7.62(\mathrm{td}, J=7.5,1.3 \mathrm{~Hz}, 1 \mathrm{H}), 7.52(\mathrm{~s}, 1 \mathrm{H}), 7.41(\mathrm{td}, J=7.7,1.6 \mathrm{~Hz}, 1 \mathrm{H})$. ${ }^{13} \mathrm{C}$-NMR (151 MHz, DMSO- $\left.d_{6}\right) \delta=142.4,141.3,139.3,138.2,134.3,134.2,132.2,130.0,129.4,129.2$, 116.0, 112.4, 47.7, 19.7, 19.3, 16.8. ${ }^{19} \mathrm{~F}-\mathrm{NMR}$ (565 MHz, DMSO- $\left.d_{6}\right) \delta=-148.3$. FTIR (ATR, neat) $\tilde{v}=$ 3627, 3543, 2987, 2926, 1622, 1483, 1456, 1437, 1390, 1286, 1257. HRMS (ESI', MeOH) $\mathrm{m} / \mathrm{z}=$ $335.02885\left[\mathrm{M}-B F_{4}\right]^{+}$. Calculated for $\mathrm{C}_{16} \mathrm{H}_{16} \mathrm{I}^{+}: \mathrm{m} / \mathrm{z}=335.02912 . \mathrm{Mp} \mathrm{T}=189-190$. 


\section{1,4,10-Trimethyl-10H-dibenzo[b,e]iodinin-5-ium tetrafluoroborate $(8 \mathrm{~g})$}

Following OPP2, 1-(2-iodophenyl)ethan-1-ol (5n, $49.6 \mathrm{mg}$ ) and p-xylene $(123 \mu \mathrm{l}, 103 \mathrm{mg})$ gave after $2 \mathrm{~h}$ for the first reaction step and $4 \mathrm{~h}$ for the second reaction step the product $8 \mathrm{~g}(16.9 \mathrm{mg}, 40.1 \mu \mathrm{mol}, 20 \%)$ as colourless solid.

${ }^{1} \mathrm{H}-\mathrm{NMR}\left(\mathrm{CD}_{3} \mathrm{OD}, 601 \mathrm{MHz}\right) \delta=8.11(\mathrm{~d}, J=8.1 \mathrm{~Hz}, 1 \mathrm{H}), 7.86(\mathrm{~d}, J=7.6 \mathrm{~Hz}$, $1 \mathrm{H}), 7.66(\mathrm{t}, J=7.5 \mathrm{~Hz}, 1 \mathrm{H}), 7.49-7.43(\mathrm{~m}, 1 \mathrm{H}), 7.42(\mathrm{~d}, J=7.7 \mathrm{~Hz}, 1 \mathrm{H}), 7.31$ (d, $J=7.7 \mathrm{~Hz}, 1 \mathrm{H}$ ), $5.08(\mathrm{q}, J=7.5 \mathrm{~Hz}, 1 \mathrm{H}), 2.62(\mathrm{~s}, 3 \mathrm{H}), 2.60(\mathrm{~s}, 3 \mathrm{H}), 1.57$ (d,

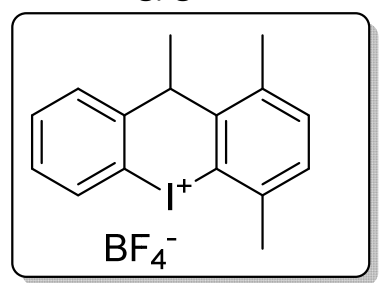
$J=7.6 \mathrm{~Hz}, 3 \mathrm{H}$ ). ${ }^{13} \mathrm{C}-\mathrm{NMR}$ (CD $\left.\mathrm{CDD}_{3}, 151 \mathrm{MHz}\right) \delta=142.9,140.7,139.3,138.2$, 135.3, 134.8, 133.8, 133.2, 131.1, 130.9, 117.2, 111.5, 47.2, 25.3, 20.3, 17.5. ${ }^{19} \mathrm{~F}-\mathrm{NMR}\left(\mathrm{CD}_{3} \mathrm{OD}\right.$, $565 \mathrm{MHz}$ ) $\delta=-155.1$. FTIR (ATR, neat) $\tilde{v}=2935,1599,1560,1472,1454,1439,1394,1019,834,764$.

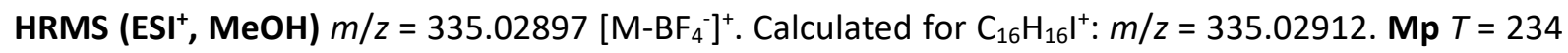
(decomp.). 


\section{OPP1}

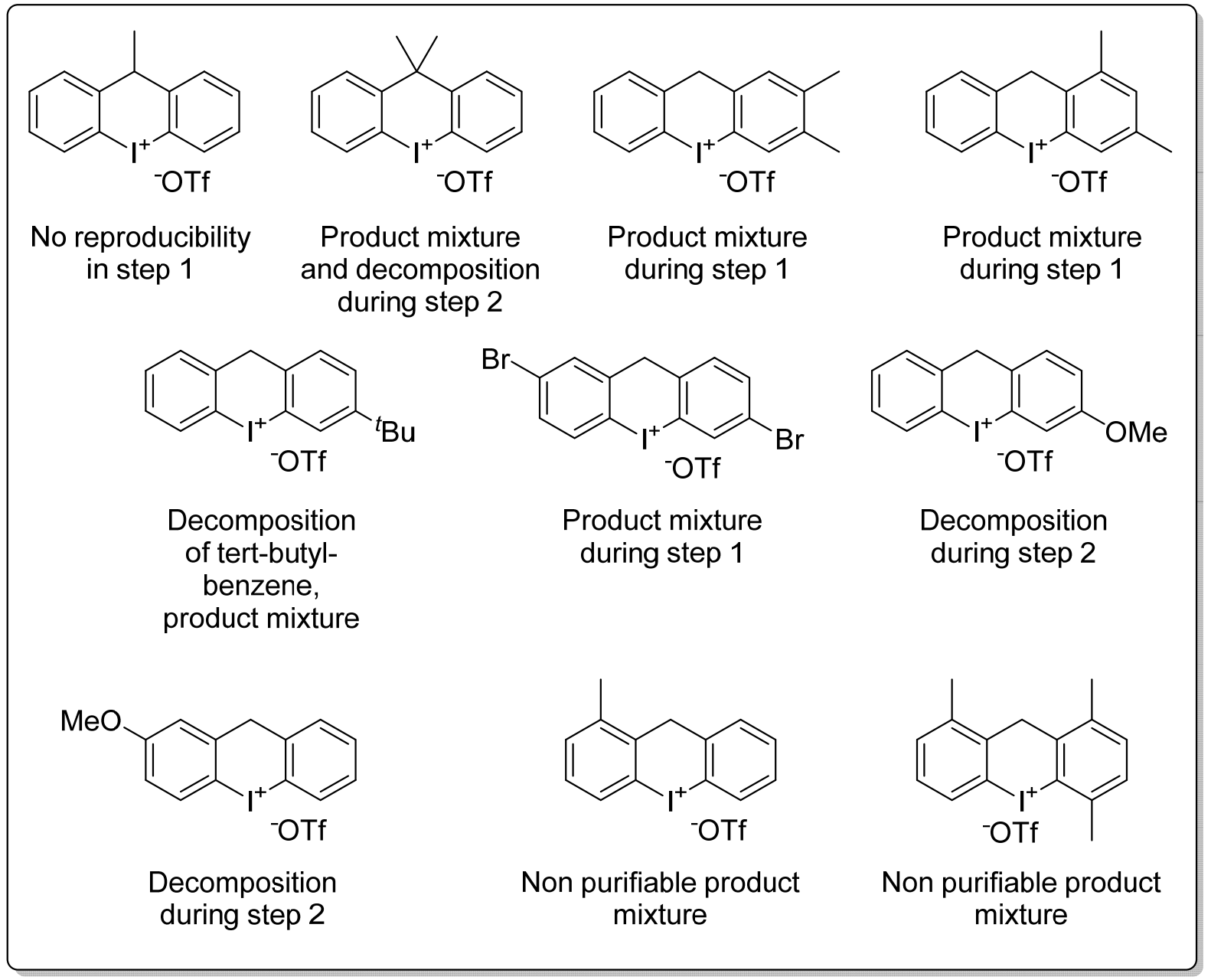

OPP2

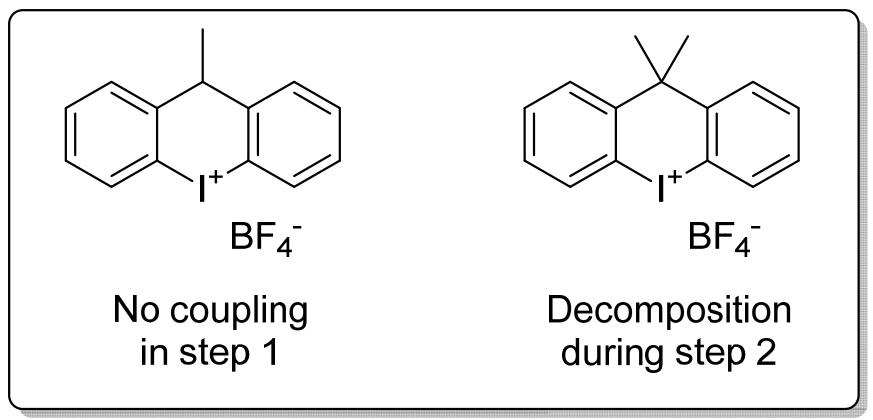

Figure S1: Substrates that could not successfully be synthesized with OPP1 or OPP2. 


\section{Computational Details}

For DFT calculations the Orca 4.2.0 program suite was utilized. Structures optimizations and frequency calculations were performed with PBEO functional ${ }^{[23]}$ and Becke-Johnson damping ${ }^{[24]}$ using the def2SVP basis set ${ }^{[25]}$ and an auxiliary basis set for the RI approximation. ${ }^{[26]}$ On the optimized structures, single point calculations were performed with DSD-BLYP functional ${ }^{[27]}$ and Becke-Johnson Damping as parametrized in GMTKN55 benchmark set ${ }^{[28]}$ using a def2-TZVPP basis set ${ }^{[25]}$ and auxiliary basis sets for the RI-approximation. ${ }^{[26,29]}$ Transition state structures contain exactly one imaginary frequency and the connection of two minima was confirmed with IRC calculations.

Table S6: Energies for iodonium salt conformers as calculated on CPCM(DCM)/RI-DSD-BLYP/TZVPP//RI-PBEO/SVP level of theory.

\begin{tabular}{cccccc}
\hline$\#$ & $\mathbf{R}$ & Conformer & $\mathbf{G} / \mathbf{E h}$ & $\mathbf{G} / \mathbf{k c a l}$ & $\begin{array}{c}\Delta \mathrm{E} / \mathbf{k c a l} \\
\text { (to axial conformer with same R-group) }\end{array}$ \\
\hline $\mathbf{1}$ & $\mathrm{Me}$ & $\mathrm{Ax}$ & -1261.6898 & -791723.0 & 0.0 \\
$\mathbf{2}$ & $\mathrm{Me}$ & $\mathrm{Eq}$ & -1261.6888 & -791722.3 & 0.6 \\
$\mathbf{3}$ & $\mathrm{Me}$ & $\mathrm{TS}$ & -1261.6697 & -791710.4 & 12.6 \\
$\mathbf{4}$ & $\mathrm{Et}$ & $\mathrm{Ax}$ & -1300.9385 & -816351.9 & 0.0 \\
$\mathbf{5}$ & $\mathrm{Et}$ & $\mathrm{Eq}$ & -1300.9357 & -816350.1 & 1.8 \\
$\mathbf{6}$ & $\mathrm{Et}$ & $\mathrm{TS}$ & -1300.9118 & -816335.2 & 16.8 \\
$\mathbf{7}$ & $\mathrm{Pr}$ & $\mathrm{Ax}$ & -1340.1866 & -840980.5 & 0.0 \\
$\mathbf{8}$ & $\mathrm{iPr}$ & $\mathrm{Eq}$ & -1340.1798 & -840976.2 & 4.3 \\
$\mathbf{9}$ & $\mathrm{Pr}$ & $\mathrm{Ts}$ & -1340.1512 & -840958.3 & 22.2 \\
$\mathbf{1 0}$ & $t \mathrm{Bu}$ & $\mathrm{Ax}$ & -1379.4280 & -865604.9 & 0 \\
$\mathbf{1 1}$ & $t \mathrm{Bu}$ & $\mathrm{Eq}$ & -1379.4179 & -865598.8 & -4 \\
$\mathbf{1 2}$ & $t \mathrm{Bu}$ & $\mathrm{Ts}$ & --- & --- & 0.0 \\
$\mathbf{1 3}$ & $\mathrm{Ph}$ & $\mathrm{Ax}$ & -1453.2043 & -911900.2 & 3.8 \\
$\mathbf{1 4}$ & $\mathrm{Ph}$ & $\mathrm{Eq}$ & -1453.1983 & -911896.4 & 11.9 \\
$\mathbf{1 5}$ & $\mathrm{Ph}$ & $\mathrm{Ts}$ & -1453.1853 & -911888.3 & \\
\hline & & & & &
\end{tabular}

Table S7: Energies for iodonium salt conformers as calculated on CPCM(DMSO)/RI-DSD-BLYP/TZVPP//RI-PBEO/SVP level of theory.

\begin{tabular}{cccccc}
\hline$\#$ & $\mathbf{R}$ & Conformer & G / Eh & G / kcal & $\begin{array}{c}\Delta \text { E / kcal } \\
\text { (of axial conformer with same R-group) }\end{array}$ \\
\hline $\mathbf{1}$ & $\mathrm{Me}$ & $\mathrm{Ax}$ & -1261.6935 & -791725.3 & 0 \\
$\mathbf{2}$ & $\mathrm{Me}$ & $\mathrm{Eq}$ & -1261.6925 & -791724.7 & 0.6 \\
$\mathbf{3}$ & $\mathrm{Me}$ & $\mathrm{TS}$ & -1261.6733 & -791712.6 & 12.6 \\
$\mathbf{4}$ & $\mathrm{Et}$ & $\mathrm{Ax}$ & -1300.9399 & -816352.8 & 0 \\
$\mathbf{5}$ & $\mathrm{Et}$ & $\mathrm{Eq}$ & -1300.9370 & -816351.0 & 1.8 \\
$\mathbf{6}$ & $\mathrm{Et}$ & $\mathrm{TS}$ & -1300.9154 & -816337.4 & 0 \\
$\mathbf{7}$ & $\mathrm{iPr}$ & $\mathrm{Ax}$ & -1340.1879 & -840981.3 & 4.2 \\
$\mathbf{8}$ & $\mathrm{PPr}$ & $\mathrm{Eq}$ & -1340.1811 & -840977.1 & 20.8 \\
$\mathbf{9}$ & $\mathrm{iPr}$ & $\mathrm{Ts}$ & -1340.1548 & -840960.5 & 0 \\
$\mathbf{1 0}$ & $t \mathrm{Bu}$ & $\mathrm{Ax}$ & -1379.4294 & -865605.7 & 6.4 \\
$\mathbf{1 1}$ & $t \mathrm{Bu}$ & $\mathrm{Eq}$ & -1379.4192 & -865599.3 &.-- \\
$\mathbf{1 2}$ & $t \mathrm{Bu}$ & $\mathrm{Ts}$ & --- & --- & 0 \\
$\mathbf{1 3}$ & $\mathrm{Ph}$ & $\mathrm{Ax}$ & -1453.2058 & -911901.2 & 10.8 \\
$\mathbf{1 4}$ & $\mathrm{Ph}$ & $\mathrm{Eq}$ & -1453.1997 & -911897.3 & 10.4 \\
$\mathbf{1 5}$ & $\mathrm{Ph}$ & $\mathrm{Ts}$ & -1453.1892 & -911890.7 & \\
\hline
\end{tabular}


Table S8: Energies for iodonium salt conformers as calculated on CPCM(MeOH)/RI-DSD-BLYP/TZVPP//RI-PBEO/SVP level of theory.

\begin{tabular}{cccccc}
\hline$\#$ & $\mathbf{R}$ & Conformer & G / Eh & $\mathbf{G ~ / ~ k c a l ~}$ & $\begin{array}{c}\Delta \mathbf{E} / \mathbf{k c a l} \\
\text { (of axial conformer with same R-group) }\end{array}$ \\
\hline $\mathbf{1}$ & $\mathrm{Me}$ & $\mathrm{Ax}$ & -1261.6931 & -791725.0 & 0.0 \\
$\mathbf{2}$ & $\mathrm{Me}$ & $\mathrm{Eq}$ & -1261.6921 & -791724.4 & 0.6 \\
$\mathbf{3}$ & $\mathrm{Me}$ & $\mathrm{TS}$ & -1261.6729 & -791712.4 & 12.6 \\
$\mathbf{4}$ & $\mathrm{Et}$ & $\mathrm{Ax}$ & -1300.9395 & -816352.5 & 0.0 \\
$\mathbf{5}$ & $\mathrm{Et}$ & $\mathrm{Eq}$ & -1300.9366 & -816350.7 & 1.8 \\
$\mathbf{6}$ & $\mathrm{Et}$ & $\mathrm{TS}$ & -1300.9150 & -816337.2 & 15.4 \\
$\mathbf{7}$ & $\mathrm{iPr}$ & $\mathrm{Ax}$ & -1340.1875 & -840981.1 & 0.0 \\
$\mathbf{8}$ & $\mathrm{PPr}$ & $\mathrm{Eq}$ & -1340.1807 & -840976.8 & 3.7 \\
$\mathbf{9}$ & $\mathrm{iPr}$ & $\mathrm{Ts}$ & -1340.1544 & -840960.3 & 20.2 \\
$\mathbf{1 0}$ & $t \mathrm{Bu}$ & $\mathrm{Ax}$ & -1379.4290 & -865605.5 & 0.0 \\
$\mathbf{1 1}$ & $t \mathrm{Bu}$ & $\mathrm{Eq}$ & -1379.4188 & -865599.1 & 6.4 \\
$\mathbf{1 2}$ & $t \mathrm{Bu}$ & $\mathrm{Ts}$ & --- & --- & 0.0 \\
$\mathbf{1 3}$ & $\mathrm{Ph}$ & $\mathrm{Ax}$ & -1453.2054 & -911900.9 & 3.8 \\
$\mathbf{1 4}$ & $\mathrm{Ph}$ & $\mathrm{Eq}$ & -1453.1993 & -911897.1 & 10.4 \\
$\mathbf{1 5}$ & $\mathrm{Ph}$ & $\mathrm{Ts}$ & -1453.1888 & -911890.5 & \\
\hline
\end{tabular}

\section{Coordinates of \#1 R = Me (8a'), axial conformer}

32

symmetry c1

C
C
C
H
C
C
C
C
C
C
H
C
H
C
H
H
I
C
H
H
C
H
C
H
H
H
B

$-3.446174000$

2.022300000

$-0.838332000$

$-2.613878000$

3.116658000

$-1.062506000$

$-3.140248000$

1.014989000

$-1.131174000$

$-5.077625000$

3.522396000

0.105188000

$-5.348447000$

1.381601000

$-0.056271000$

$-2.972706000$

4.424432000

$-0.726541000$

$-4.686539000$

2.230362000

$-0.240167000$

$-1.495713000$

5.670141000

$-2.353870000$

$-0.825312000$

4.616776000

$-2.974766000$

$-0.230592000$

4.687203000

$-4.225886000$

$-0.318033000$

5.894057000

$-4.915973000$

0.320858000

3.842925000

$-4.639237000$

$-0.994664000$

6.972463000

$-4.352607000$

0.156598000

5.981075000

$-5.895017000$

$-1.574351000$

6.857319000

$-3.092544000$

$-1.064990000$

7.917922000

$-4.894535000$

$-2.092656000$

7.711881000

$-2.649378000$

$-0.715603000$

2.776114000

$-1.924710000$

$-4.233244000$

4.601004000

$-0.143443000$

$-4.550742000$

5.612546000

0.123825000

$-6.052202000$

3.693405000

0.567349000

$-2.062935000$

5.613057000

$-0.951663000$

$-2.696282000$

6.505767000

$-0.842014000$

$-0.952111000$

5.715009000

0.106053000

$-0.217762000$

4.900571000

0.028657000

$-1.386560000$

5.698017000

1.116094000

$-0.399706000$

6.655992000

$-0.026175000$

2.550074000

3.390372000

$-2.740356000$ 


$\begin{array}{llll}\text { F } & 1.452833000 & 2.481828000 & -3.158607000 \\ \text { F } & 2.846915000 & 4.184277000 & -3.828248000 \\ \text { F } & 2.000018000 & 4.147901000 & -1.692449000 \\ \text { F } & 3.598907000 & 2.618136000 & -2.318956000\end{array}$

Coordinates of \#2 R = Me (8a'), equatorial conformer

32

symmetry c1

$\begin{array}{llll}\text { C } & -2.513554000 & 0.811780000 & -1.109222000 \\ \text { C } & -2.275715000 & 2.150828000 & -0.849718000 \\ \text { H } & -1.779285000 & 0.194606000 & -1.626241000 \\ \text { C } & -4.606166000 & 1.073335000 & 0.060082000 \\ \text { H } & -3.918478000 & -0.786057000 & -0.807357000 \\ \text { C } & -3.126884000 & 2.995023000 & -0.134966000 \\ \text { C } & -3.707477000 & 0.270972000 & -0.635828000 \\ \text { C } & -2.552656000 & 5.198337000 & -1.147721000 \\ \text { C } & -1.599230000 & 4.768036000 & -2.078522000 \\ \text { C } & -1.369420000 & 5.412989000 & -3.289118000 \\ \text { C } & -2.117128000 & 6.549163000 & -3.593146000 \\ \text { H } & -0.624833000 & 5.037162000 & -3.994508000 \\ \text { C } & -3.080106000 & 7.000327000 & -2.694186000 \\ \text { H } & -1.950206000 & 7.071975000 & -4.537162000 \\ \text { C } & -3.296930000 & 6.331504000 & -1.490494000 \\ \text { H } & -3.674255000 & 7.885569000 & -2.931126000 \\ \text { H } & -4.056646000 & 6.704874000 & -0.802486000 \\ \text { I } & -0.493302000 & 3.037429000 & -1.575554000 \\ \text { C } & -4.319719000 & 2.414622000 & 0.307844000 \\ \text { H } & -5.030596000 & 3.017631000 & 0.874080000 \\ \text { H } & -5.540854000 & 0.648180000 & 0.431735000 \\ \text { C } & -2.739883000 & 4.436606000 & 0.154570000 \\ \text { C } & -3.648254000 & 5.129512000 & 1.153983000 \\ \text { H } & -4.679464000 & 5.228415000 & 0.783243000 \\ \text { H } & -3.270961000 & 6.136614000 & 1.379945000 \\ \text { H } & -3.678376000 & 4.564499000 & 2.095662000 \\ \text { H } & -1.734358000 & 4.375065000 & 0.617667000 \\ \text { B } & 0.511034000 & 0.468317000 & 0.432094000 \\ \text { F } & -0.400349000 & -0.547262000 & 0.637008000 \\ \text { F } & 0.480024000 & 0.790275000 & -1.016853000 \\ \text { F } & 0.098864000 & 1.652072000 & 1.066940000 \\ \text { F } & 1.795233000 & 0.128987000 & 0.761300000\end{array}$

Coordinates of \#3 $R=$ Me $\left(8 a^{\prime}\right)$, transition state conformer

32

symmetry c1

$\begin{array}{llll}\text { C } & -2.472238000 & 2.047526000 & -0.218552000 \\ \text { C } & -2.250797000 & 3.189601000 & -0.993119000 \\ \text { H } & -1.831229000 & 1.168950000 & -0.332856000 \\ \text { C } & -4.337947000 & 3.147889000 & 0.794331000 \\ \text { H } & -3.696578000 & 1.139864000 & 1.303352000 \\ \text { C } & -3.004399000 & 4.359740000 & -0.879238000 \\ \text { C } & -3.518487000 & 2.027336000 & 0.693081000\end{array}$




$\begin{array}{llll}\text { C } & -1.497649000 & 5.899573000 & -2.425997000 \\ \text { C } & -0.521272000 & 4.968510000 & -2.793858000 \\ \text { C } & 0.675924000 & 5.276174000 & -3.438030000 \\ \text { C } & 0.913227000 & 6.594242000 & -3.806459000 \\ \text { H } & 1.403960000 & 4.492734000 & -3.657005000 \\ \text { C } & -0.055825000 & 7.555249000 & -3.540289000 \\ \text { H } & 1.845182000 & 6.851888000 & -4.312855000 \\ \text { C } & -1.217512000 & 7.209633000 & -2.861453000 \\ \text { H } & 0.091423000 & 8.591106000 & -3.853114000 \\ \text { H } & -1.944308000 & 7.997029000 & -2.667431000 \\ \text { I } & -0.827319000 & 2.902461000 & -2.506639000 \\ \text { C } & -4.076356000 & 4.278426000 & 0.030402000 \\ \text { H } & -4.746079000 & 5.130610000 & 0.141643000 \\ \text { H } & -5.189033000 & 3.149039000 & 1.478431000 \\ \text { C } & -2.795901000 & 5.673888000 & -1.639103000 \\ \text { H } & -3.611773000 & 5.718606000 & -2.386476000 \\ \text { C } & -2.994189000 & 6.847915000 & -0.660828000 \\ \text { H } & -2.178399000 & 6.868303000 & 0.076861000 \\ \text { H } & -3.942559000 & 6.776173000 & -0.119125000 \\ \text { H } & -3.015903000 & 7.814374000 & -1.174320000 \\ \text { B } & 1.763384000 & 1.720723000 & -3.983415000 \\ \text { F } & 0.693190000 & 2.526713000 & -4.572057000 \\ \text { F } & 2.400982000 & 2.575365000 & -3.071678000 \\ \text { F } & 1.117519000 & 0.688605000 & -3.289710000 \\ \text { F } & 2.598089000 & 1.270250000 & -4.962750000 \\ & & & \end{array}$

\section{Coordinates of \#4 R = Et $\left(8 b^{\prime}\right)$, axial conformer}

35

\section{symmetry c1}

$\begin{array}{llll}\text { C } & -3.478680000 & 1.998848000 & -0.879018000 \\ \mathbf{C} & -2.646055000 & 3.096511000 & -1.085222000 \\ \mathbf{H} & -3.165669000 & 0.993881000 & -1.172420000 \\ \mathbf{C} & -5.129118000 & 3.490660000 & 0.043717000 \\ \mathbf{H} & -5.390935000 & 1.349497000 & -0.128981000 \\ \mathbf{C} & -3.012469000 & 4.402191000 & -0.748989000 \\ \mathbf{C} & -4.728728000 & 2.200874000 & -0.299263000 \\ \mathbf{C} & -1.523409000 & 5.659395000 & -2.350301000 \\ \mathbf{C} & -0.841932000 & 4.611885000 & -2.969309000 \\ \mathbf{C} & -0.232063000 & 4.691644000 & -4.212556000 \\ \mathbf{C} & -0.315352000 & 5.902272000 & -4.896572000 \\ \mathbf{H} & 0.327412000 & 3.851768000 & -4.624160000 \\ \mathbf{C} & -1.002472000 & 6.975128000 & -4.335121000 \\ \mathbf{H} & 0.170778000 & 5.996699000 & -5.869265000 \\ \mathbf{C} & -1.596744000 & 6.850787000 & -3.082813000 \\ \mathbf{H} & -1.069404000 & 7.923514000 & -4.872362000 \\ \mathbf{H} & -2.122931000 & 7.700881000 & -2.640413000 \\ \mathbf{I} & -0.738010000 & 2.764997000 & -1.928431000 \\ \mathbf{C} & -4.283734000 & 4.572382000 & -0.187169000 \\ \mathbf{H} & -4.607657000 & 5.581763000 & 0.079541000 \\ \mathbf{H} & -6.111435000 & 3.657409000 & 0.490783000 \\ \mathbf{C} & -2.100875000 & 5.591993000 & -0.954541000 \\ \mathbf{H} & -2.734169000 & 6.484736000 & -0.836479000\end{array}$




$\begin{array}{llll}\mathbf{C} & -0.981578000 & 5.702164000 & 0.105671000 \\ \mathbf{H} & -0.242593000 & 4.896639000 & -0.030450000 \\ \mathbf{H} & -0.430017000 & 6.630738000 & -0.111139000 \\ \mathbf{B} & 2.536045000 & 3.387950000 & -2.701431000 \\ \mathbf{F} & 1.446832000 & 2.479602000 & -3.138677000 \\ \mathbf{F} & 2.845027000 & 4.188944000 & -3.780853000 \\ \mathbf{F} & 1.971277000 & 4.138281000 & -1.655890000 \\ \mathbf{F} & 3.581566000 & 2.616011000 & -2.270879000 \\ \mathbf{C} & -1.495262000 & 5.712776000 & 1.533724000 \\ \mathbf{H} & -1.993920000 & 4.767272000 & 1.796752000 \\ \mathbf{H} & -0.665816000 & 5.858648000 & 2.240673000 \\ \mathbf{H} & -2.219785000 & 6.527051000 & 1.698224000\end{array}$

\section{Coordinates of \#5 R = Et $\left(8 b^{\prime}\right)$, equatorial conformer}

35

\begin{tabular}{llll} 
symmetry c1 & & & \\
C & -2.505130000 & 0.790363000 & -1.188070000 \\
C & -2.269350000 & 2.118558000 & -0.875294000 \\
H & -1.750771000 & 0.182325000 & -1.686768000 \\
C & -4.662345000 & 1.047636000 & -0.140243000 \\
H & -3.942959000 & -0.797393000 & -1.012977000 \\
C & -3.146526000 & 2.949685000 & -0.177731000 \\
C & -3.729728000 & 0.251449000 & -0.798942000 \\
C & -2.574066000 & 5.158465000 & -1.131235000 \\
C & -1.598027000 & 4.754788000 & -2.050974000 \\
C & -1.368004000 & 5.410028000 & -3.255731000 \\
C & -2.142963000 & 6.526387000 & -3.565753000 \\
H & -0.604266000 & 5.057131000 & -3.952374000 \\
C & -3.133546000 & 6.945688000 & -2.681016000 \\
H & -1.977164000 & 7.057449000 & -4.505362000 \\
C & -3.349183000 & 6.267947000 & -1.482041000 \\
H & -3.750988000 & 7.812489000 & -2.926522000 \\
H & -4.131955000 & 6.613402000 & -0.805040000 \\
I & -0.475539000 & 3.034611000 & -1.538320000 \\
C & -4.375489000 & 2.376541000 & 0.165206000 \\
H & -5.115469000 & 2.975884000 & 0.697755000 \\
H & -5.625659000 & 0.626472000 & 0.155053000 \\
C & -2.751693000 & 4.378700000 & 0.159850000 \\
C & -3.625257000 & 5.060399000 & 1.206683000 \\
H & -4.680209000 & 5.059876000 & 0.883207000 \\
H & -3.328467000 & 6.120280000 & 1.252981000 \\
H & -1.738967000 & 4.297475000 & 0.604604000 \\
B & 0.475627000 & 0.467855000 & 0.490515000 \\
F & -0.457515000 & -0.534146000 & 0.662266000 \\
F & 0.499613000 & 0.792351000 & -0.957127000 \\
F & 0.058453000 & 1.656487000 & 1.114405000 \\
F & 1.742215000 & 0.109042000 & 0.864390000 \\
C & -3.485390000 & 4.450533000 & 2.593976000 \\
H & -3.758886000 & 3.385850000 & 2.612637000 \\
H & -2.445817000 & 4.520892000 & 2.948878000 \\
H & -4.123826000 & 4.975792000 & 3.319089000 \\
& & & \\
\hline
\end{tabular}


Coordinates of \#6 R = Et $\left(8 b^{\prime}\right)$, transition state conformer

35

symmetry c1

\begin{tabular}{llll} 
C & -2.355584000 & 1.636939000 & -0.425088000 \\
C & -2.082586000 & 2.888537000 & -0.985308000 \\
H & -1.850353000 & 0.741104000 & -0.796742000 \\
C & -3.928723000 & 2.684428000 & 1.045705000 \\
H & -3.497774000 & 0.559169000 & 1.047563000 \\
C & -2.663293000 & 4.073765000 & -0.535379000 \\
C & -3.280350000 & 1.532960000 & 0.604530000 \\
C & -1.218914000 & 5.763141000 & -1.972831000 \\
C & -0.414670000 & 4.856758000 & -2.666407000 \\
C & 0.694758000 & 5.204839000 & -3.436268000 \\
C & 1.000579000 & 6.550916000 & -3.589473000 \\
H & 1.299708000 & 4.433943000 & -3.917052000 \\
C & 0.187799000 & 7.506384000 & -2.986668000 \\
H & 1.863885000 & 6.839721000 & -4.191694000 \\
C & -0.882607000 & 7.112921000 & -2.194780000 \\
H & 0.390910000 & 8.570626000 & -3.122950000 \\
H & -1.491711000 & 7.887193000 & -1.726603000 \\
I & -0.877563000 & 2.798053000 & -2.702632000 \\
C & -3.616832000 & 3.917694000 & 0.489126000 \\
H & -4.140070000 & 4.800089000 & 0.860645000 \\
H & -4.680837000 & 2.625413000 & 1.835137000 \\
C & -2.405558000 & 5.488138000 & -1.048376000 \\
H & -3.297958000 & 5.732273000 & -1.656818000 \\
C & -2.390026000 & 6.489153000 & 0.150799000 \\
H & -2.398955000 & 5.923606000 & 1.092611000 \\
H & -1.429268000 & 7.021596000 & 0.162555000 \\
B & 1.351298000 & 1.735693000 & -4.754425000 \\
F & 0.312865000 & 2.733362000 & -5.012298000 \\
F & 2.218186000 & 2.339610000 & -3.833783000 \\
F & 0.690624000 & 0.659092000 & -4.143947000 \\
F & 1.965987000 & 1.393754000 & -5.922506000 \\
C & -3.534738000 & 7.489438000 & 0.142429000 \\
H & -4.515483000 & 6.988483000 & 0.182859000 \\
H & -3.473501000 & 8.170093000 & 1.004579000 \\
H & -3.531124000 & 8.106913000 & -0.770131000 \\
& & & \\
\hline
\end{tabular}

Coordinates of \#7 R = $i \operatorname{Pr}\left(8 c^{\prime}\right)$, axial conformer

38

symmetry $\mathrm{c} 1$

$\begin{array}{llll}\text { C } & -3.480999000 & 2.008879000 & -0.881025000 \\ \text { C } & -2.642146000 & 3.103607000 & -1.075613000 \\ \text { H } & -3.164894000 & 1.002019000 & -1.164105000 \\ \text { C } & -5.145995000 & 3.509956000 & -0.000022000 \\ \text { H } & -5.410275000 & 1.368592000 & -0.167338000 \\ \text { C } & -3.010283000 & 4.412500000 & -0.750307000 \\ \text { C } & -4.742098000 & 2.217198000 & -0.327866000 \\ \text { C } & -1.539727000 & 5.648992000 & -2.357158000\end{array}$




$\begin{array}{llll}\text { C } & -0.855654000 & 4.599360000 & -2.968185000 \\ \text { C } & -0.276097000 & 4.657845000 & -4.226772000 \\ \text { C } & -0.398621000 & 5.848739000 & -4.938675000 \\ \text { H } & 0.287783000 & 3.817443000 & -4.630751000 \\ \text { C } & -1.096491000 & 6.920830000 & -4.388815000 \\ \text { H } & 0.062951000 & 5.928486000 & -5.924542000 \\ \text { C } & -1.658355000 & 6.817692000 & -3.119817000 \\ \text { H } & -1.196262000 & 7.853137000 & -4.948621000 \\ \text { H } & -2.189154000 & 7.668935000 & -2.686104000 \\ \text { I } & -0.719213000 & 2.777867000 & -1.890330000 \\ \text { C } & -4.293008000 & 4.588486000 & -0.217176000 \\ \text { H } & -4.618562000 & 5.599758000 & 0.039804000 \\ \text { H } & -6.137228000 & 3.681422000 & 0.425092000 \\ \text { C } & -2.087528000 & 5.594890000 & -0.948790000 \\ \text { H } & -2.704625000 & 6.498576000 & -0.822014000 \\ \text { C } & -0.968857000 & 5.688771000 & 0.130340000 \\ \text { H } & -0.336826000 & 4.787779000 & 0.062444000 \\ \text { B } & 2.526072000 & 3.605686000 & -2.607052000 \\ \text { F } & 1.517739000 & 2.601215000 & -3.024576000 \\ \text { F } & 2.769110000 & 4.409481000 & -3.701865000 \\ \text { F } & 1.902239000 & 4.331381000 & -1.576655000 \\ \text { F } & 3.632842000 & 2.934353000 & -2.161933000 \\ \text { C } & -1.575450000 & 5.738247000 & 1.526615000 \\ \text { H } & -2.181866000 & 4.848495000 & 1.751646000 \\ \text { H } & -0.780646000 & 5.801279000 & 2.284244000 \\ \text { H } & -2.219522000 & 6.625780000 & 1.647781000 \\ \text { C } & -0.062905000 & 6.885062000 & -0.128068000 \\ \text { H } & 0.692098000 & 6.962635000 & 0.667568000 \\ \text { H } & 0.477631000 & 6.790331000 & -1.079756000 \\ \text { H } & -0.637966000 & 7.826702000 & -0.140160000\end{array}$

\section{Coordinates of \#8 $\mathrm{R}=i \operatorname{Pr}\left(8 \mathrm{c}^{\prime}\right)$, equatorial conformer}

38

$\begin{array}{llll}\text { symmetry c1 } & & & \\ \text { C } & -2.488679000 & 0.848695000 & -1.203551000 \\ \text { C } & -2.246194000 & 2.170910000 & -0.872211000 \\ \text { H } & -1.724862000 & 0.232682000 & -1.677551000 \\ \text { C } & -4.690664000 & 1.141654000 & -0.259467000 \\ \text { H } & -3.961816000 & -0.713295000 & -1.102772000 \\ \text { C } & -3.135623000 & 3.008088000 & -0.198518000 \\ \text { C } & -3.739019000 & 0.330109000 & -0.872311000 \\ \text { C } & -2.596385000 & 5.173803000 & -1.169402000 \\ \text { C } & -1.602335000 & 4.783157000 & -2.076257000 \\ \text { C } & -1.423304000 & 5.382751000 & -3.318305000 \\ \text { C } & -2.278479000 & 6.419965000 & -3.685478000 \\ \text { H } & -0.643112000 & 5.041257000 & -4.002153000 \\ \text { C } & -3.306338000 & 6.803956000 & -2.826423000 \\ \text { H } & -2.153643000 & 6.908581000 & -4.654017000 \\ \text { C } & -3.468386000 & 6.183354000 & -1.589270000 \\ \text { H } & -3.996765000 & 7.596030000 & -3.124381000 \\ \text { H } & -4.286410000 & 6.493170000 & -0.936619000 \\ \text { I } & -0.444955000 & 3.097079000 & -1.512610000\end{array}$




$\begin{array}{llll}\mathbf{C} & -4.395040000 & 2.462527000 & 0.070897000 \\ \mathbf{H} & -5.150067000 & 3.077461000 & 0.563696000 \\ \mathbf{H} & -5.677247000 & 0.738157000 & -0.021848000 \\ \mathbf{C} & -2.716668000 & 4.426395000 & 0.148009000 \\ \mathbf{C} & -3.497949000 & 5.115892000 & 1.274548000 \\ \mathbf{H} & -4.551428000 & 5.240911000 & 0.961481000 \\ \mathbf{H} & -1.683450000 & 4.329977000 & 0.538074000 \\ \mathbf{B} & 0.439442000 & 0.600500000 & 0.625878000 \\ \mathbf{F} & -0.504912000 & -0.392434000 & 0.786752000 \\ \mathbf{F} & 0.524035000 & 0.884971000 & -0.828273000 \\ \mathbf{F} & 0.003213000 & 1.807127000 & 1.201514000 \\ \mathbf{F} & 1.687920000 & 0.247116000 & 1.060903000 \\ \mathbf{C} & -3.457779000 & 4.277700000 & 2.552644000 \\ \mathbf{H} & -3.896319000 & 3.279059000 & 2.433422000 \\ \mathbf{H} & -2.416319000 & 4.134726000 & 2.882294000 \\ \mathbf{H} & -3.998279000 & 4.791043000 & 3.361539000 \\ \mathbf{C} & -2.910166000 & 6.493830000 & 1.578878000 \\ \mathbf{H} & -3.475586000 & 6.978963000 & 2.388100000 \\ \mathbf{H} & -1.867053000 & 6.393706000 & 1.920089000 \\ \mathbf{H} & -2.910478000 & 7.171796000 & 0.715150000\end{array}$

Coordinates of \#9 $\mathrm{R}=i \operatorname{Pr}\left(8 c^{\prime}\right)$, transition state conformer

38

$\begin{array}{lrll}\text { symmetry c1 } & & & \\ \text { C } & -1.983575000 & 1.699641000 & -0.275041000 \\ \text { C } & -1.884832000 & 2.932488000 & -0.925501000 \\ \text { H } & -1.391677000 & 0.847098000 & -0.619158000 \\ \text { C } & -3.635268000 & 2.641750000 & 1.165569000 \\ \text { H } & -2.931200000 & 0.595886000 & 1.316027000 \\ \text { C } & -2.572163000 & 4.085130000 & -0.526992000 \\ \text { C } & -2.851050000 & 1.553641000 & 0.798386000 \\ \text { C } & -1.230109000 & 5.778183000 & -2.050501000 \\ \text { C } & -0.407492000 & 4.871444000 & -2.729033000 \\ \text { C } & 0.713309000 & 5.206051000 & -3.486854000 \\ \text { C } & 1.033306000 & 6.548489000 & -3.642343000 \\ \text { H } & 1.317193000 & 4.428971000 & -3.961331000 \\ \text { C } & 0.213339000 & 7.505453000 & -3.056353000 \\ \text { H } & 1.906820000 & 6.829724000 & -4.233286000 \\ \text { C } & -0.875163000 & 7.120006000 & -2.283769000 \\ \text { H } & 0.420020000 & 8.569405000 & -3.190272000 \\ \text { H } & -1.482684000 & 7.901366000 & -1.841876000 \\ \text { I } & -0.871627000 & 2.819754000 & -2.757366000 \\ \text { C } & -3.491390000 & 3.863735000 & 0.518299000 \\ \text { H } & -4.150282000 & 4.668658000 & 0.841241000 \\ \text { H } & -4.370885000 & 2.547282000 & 1.967050000 \\ \text { C } & -2.439551000 & 5.492309000 & -1.146490000 \\ \text { H } & -3.330389000 & 5.609247000 & -1.794480000 \\ \text { C } & -2.586699000 & 6.559336000 & -0.003201000 \\ \text { H } & -3.307718000 & 6.113816000 & 0.685618000 \\ \text { B } & 1.200650000 & 1.861397000 & -5.082859000 \\ \text { F } & 0.085009000 & 2.813366000 & -5.127415000 \\ \text { F } & 2.209477000 & 2.506159000 & -4.357454000\end{array}$




$\begin{array}{llll}\mathbf{F} & 0.721816000 & 0.760809000 & -4.359798000 \\ \mathbf{F} & 1.577571000 & 1.544033000 & -6.354399000 \\ \mathbf{C} & -3.257021000 & 7.888871000 & -0.375366000 \\ \mathbf{H} & -4.002968000 & 7.763914000 & -1.175385000 \\ \mathbf{H} & -3.785088000 & 8.285775000 & 0.505045000 \\ \mathbf{H} & -2.560333000 & 8.680734000 & -0.685013000 \\ \mathbf{C} & -1.311615000 & 6.734131000 & 0.814565000 \\ \mathbf{H} & -1.516883000 & 7.336542000 & 1.712476000 \\ \mathbf{H} & -0.920578000 & 5.760109000 & 1.147325000 \\ \mathbf{H} & -0.513057000 & 7.237710000 & 0.251376000\end{array}$

\section{Coordinates of \#10 $R=t B u$, axial conformer}

41 symmetry c1

\begin{tabular}{|c|c|c|c|}
\hline C & -3.425270000 & 1.913711000 & -0.980925000 \\
\hline C & -2.591495000 & 3.014237000 & -1.180774000 \\
\hline H & -3.136478000 & 0.921942000 & -1.337768000 \\
\hline C & -5.020583000 & 3.368809000 & 0.076298000 \\
\hline H & -5.302113000 & 1.236014000 & -0.170548000 \\
\hline C & -2.924954000 & 4.306598000 & -0.764888000 \\
\hline C & -4.643633000 & 2.091581000 & -0.333821000 \\
\hline C & -1.432657000 & 5.619838000 & -2.333468000 \\
\hline C & -0.793055000 & 4.594136000 & -3.030378000 \\
\hline C & -0.191556000 & 4.735630000 & -4.274621000 \\
\hline C & -0.234802000 & 5.985938000 & -4.882687000 \\
\hline H & 0.329376000 & 3.903370000 & -4.747979000 \\
\hline C & -0.892525000 & 7.039210000 & -4.252865000 \\
\hline H & 0.248383000 & 6.122525000 & -5.851788000 \\
\hline C & -1.482355000 & 6.850390000 & -3.008879000 \\
\hline H & -0.944469000 & 8.019490000 & -4.731159000 \\
\hline H & -1.992960000 & 7.684202000 & -2.521397000 \\
\hline I & -0.767182000 & 2.652023000 & -2.178976000 \\
\hline C & -4.177681000 & 4.451372000 & -0.148484000 \\
\hline H & -4.489929000 & 5.450304000 & 0.164864000 \\
\hline H & -5.983213000 & 3.525688000 & 0.567481000 \\
\hline C & -2.053339000 & 5.529855000 & -0.954361000 \\
\hline H & -2.750760000 & 6.380590000 & -0.912390000 \\
\hline C & -1.057501000 & 5.817866000 & 0.237586000 \\
\hline B & 2.515800000 & 3.054648000 & -3.177154000 \\
\hline $\mathbf{F}$ & 1.322405000 & 2.265271000 & -3.556809000 \\
\hline $\mathbf{F}$ & 2.837688000 & 3.842054000 & -4.263998000 \\
\hline $\mathbf{F}$ & 2.089094000 & 3.841908000 & -2.092092000 \\
\hline $\mathbf{F}$ & 3.507947000 & 2.183624000 & -2.815347000 \\
\hline C & -1.877433000 & 5.976323000 & 1.520905000 \\
\hline H & -2.382490000 & 5.042123000 & 1.807380000 \\
\hline H & -1.212229000 & 6.259759000 & 2.350261000 \\
\hline H & -2.640850000 & 6.765323000 & 1.423704000 \\
\hline C & -0.326763000 & 7.133402000 & -0.039154000 \\
\hline H & 0.302860000 & 7.392546000 & 0.825206000 \\
\hline H & 0.329835000 & 7.061441000 & -0.917825000 \\
\hline H & -1.030587000 & 7.966158000 & -0.198590000 \\
\hline C & -0.019488000 & 4.718907000 & 0.452377000 \\
\hline
\end{tabular}




$\begin{array}{lrrl}\mathbf{H} & 0.609015000 & 4.982820000 & 1.317023000 \\ \mathbf{H} & -0.491975000 & 3.752714000 & 0.687813000 \\ \mathbf{H} & 0.658848000 & 4.594641000 & -0.404274000\end{array}$

\section{Coordinates of \#11 $R=t B u$, equatorial conformer}

\begin{tabular}{|c|c|c|c|}
\hline \multicolumn{4}{|c|}{41} \\
\hline \multicolumn{4}{|c|}{ symmetry c1 } \\
\hline C & -2.403908000 & 0.804688000 & -1.297850000 \\
\hline C & -2.254903000 & 2.139705000 & -0.962030000 \\
\hline H & -1.595100000 & 0.243668000 & -1.765278000 \\
\hline C & -4.612793000 & 0.937455000 & -0.349872000 \\
\hline H & -3.762924000 & -0.859045000 & -1.207433000 \\
\hline C & -3.202791000 & 2.924416000 & -0.300480000 \\
\hline C & -3.613850000 & 0.196658000 & -0.973925000 \\
\hline C & -2.701140000 & 5.144213000 & -1.247988000 \\
\hline C & -1.667060000 & 4.783147000 & -2.125675000 \\
\hline C & -1.434388000 & 5.409701000 & -3.345183000 \\
\hline C & -2.267649000 & 6.459494000 & -3.724456000 \\
\hline H & -0.623868000 & 5.082536000 & -4.000132000 \\
\hline C & -3.318633000 & 6.833549000 & -2.891185000 \\
\hline H & -2.102188000 & 6.969699000 & -4.675594000 \\
\hline C & -3.535964000 & 6.181948000 & -1.678441000 \\
\hline H & -3.987189000 & 7.644425000 & -3.188493000 \\
\hline H & -4.369810000 & 6.494635000 & -1.053941000 \\
\hline I & -0.473548000 & 3.140722000 & -1.525478000 \\
\hline C & -4.412179000 & 2.276247000 & -0.019944000 \\
\hline H & -5.207221000 & 2.816551000 & 0.490495000 \\
\hline H & -5.564788000 & 0.464834000 & -0.099163000 \\
\hline C & -2.849781000 & 4.364875000 & 0.053070000 \\
\hline C & -3.583531000 & 5.027669000 & 1.251256000 \\
\hline H & -1.814650000 & 4.278245000 & 0.437559000 \\
\hline B & 0.354835000 & 0.784227000 & 0.794892000 \\
\hline $\mathbf{F}$ & -0.553527000 & -0.245697000 & 0.925109000 \\
\hline $\mathbf{F}$ & 0.542235000 & 1.015155000 & -0.658707000 \\
\hline $\mathbf{F}$ & -0.179710000 & 1.988485000 & 1.289394000 \\
\hline $\mathbf{F}$ & 1.580669000 & 0.509214000 & 1.338658000 \\
\hline C & -3.441448000 & 4.084213000 & 2.457251000 \\
\hline H & -4.044269000 & 3.172088000 & 2.360796000 \\
\hline H & -2.395395000 & 3.768916000 & 2.595318000 \\
\hline H & -3.765639000 & 4.603437000 & 3.371819000 \\
\hline C & -2.827347000 & 6.316722000 & 1.602614000 \\
\hline H & -3.301049000 & 6.805066000 & 2.467786000 \\
\hline H & -1.782136000 & 6.099950000 & 1.873493000 \\
\hline H & -2.819552000 & 7.038271000 & 0.772570000 \\
\hline C & -5.072414000 & 5.367435000 & 1.021146000 \\
\hline H & -5.555444000 & 4.744542000 & 0.256610000 \\
\hline H & -5.637818000 & 5.240698000 & 1.956620000 \\
\hline H & -5.212849000 & 6.415337000 & 0.718268000 \\
\hline
\end{tabular}

Coordinates of \#12 $\mathrm{R}=t \mathrm{Bu}$, transition state conformer

A transition state for $\mathrm{R}=t \mathrm{Bu}$ could not be found. 


\section{Coordinates of \#13 R = Ph (8d'), axial conformer}

\begin{tabular}{lrll} 
39 & & & \\
Symmetry c1 & & & \\
C & -3.436390000 & 1.995177000 & -0.895504000 \\
C & -2.587162000 & 3.084840000 & -1.061763000 \\
H & -3.119022000 & 0.986983000 & -1.172024000 \\
C & -5.123564000 & 3.505617000 & -0.066345000 \\
H & -5.390000000 & 1.366317000 & -0.238957000 \\
C & -2.963597000 & 4.392540000 & -0.744776000 \\
C & -4.713373000 & 2.211739000 & -0.379846000 \\
C & -1.476992000 & 5.640400000 & -2.317862000 \\
C & -0.826453000 & 4.585401000 & -2.959927000 \\
C & -0.284713000 & 4.653034000 & -4.233588000 \\
C & -0.407727000 & 5.858923000 & -4.922505000 \\
H & 0.254048000 & 3.810527000 & -4.666455000 \\
C & -1.064159000 & 6.936795000 & -4.335487000 \\
H & 0.026148000 & 5.944691000 & -5.920408000 \\
C & -1.592607000 & 6.825316000 & -3.051337000 \\
H & -1.157527000 & 7.880505000 & -4.876958000 \\
H & -2.091653000 & 7.679863000 & -2.587228000 \\
I & -0.661030000 & 2.771160000 & -1.874763000 \\
C & -4.259107000 & 4.582080000 & -0.255155000 \\
H & -4.585594000 & 5.595525000 & -0.007358000 \\
H & -6.127491000 & 3.680228000 & 0.326486000 \\
C & -2.001873000 & 5.550500000 & -0.902597000 \\
H & -2.578222000 & 6.471711000 & -0.722782000 \\
B & 2.574953000 & 3.291247000 & -3.018703000 \\
F & 1.420937000 & 2.406250000 & -3.284238000 \\
F & 2.721572000 & 4.107029000 & -4.126325000 \\
F & 2.194652000 & 4.036222000 & -1.891933000 \\
F & 3.670046000 & 2.507109000 & -2.772341000 \\
C & -0.885422000 & 5.497444000 & 0.144701000 \\
C & -1.184052000 & 5.097472000 & 1.453510000 \\
H & 0.424381000 & 5.870329000 & -0.165823000 \\
C & -0.191903000 & 5.072041000 & 2.429074000 \\
C & 1.420085000 & 5.832054000 & 0.808509000 \\
H & 1.115900000 & 5.436091000 & 2.107655000 \\
& -2.201610000 & 4.794204000 & 1.712159000 \\
\hline & 0.691970000 & 6.160275000 & -1.182837000 \\
H & -0.440358000 & 4.756258000 & 3.445170000 \\
\hline & 2.443563000 & 6.093472000 & 0.533463000 \\
H & 1.898924000 & 5.399638000 & 2.868483000
\end{tabular}

Coordinates of \#14 R = Ph (8d'), equatorial conformer

39

$\begin{array}{llll}\text { symmetry c1 } & & & \\ \text { C } & -2.478933000 & 0.749669000 & -1.099763000 \\ \text { C } & -2.325438000 & 2.110448000 & -0.889554000 \\ \text { H } & -1.712646000 & 0.165292000 & -1.608373000 \\ \text { C } & -4.545722000 & 0.904696000 & 0.124123000\end{array}$




\begin{tabular}{|c|c|c|c|}
\hline H & -3.759961000 & -0.931271000 & -0.712080000 \\
\hline C & -3.221042000 & 2.925409000 & -0.193722000 \\
\hline C & -3.619775000 & 0.143026000 & -0.580258000 \\
\hline C & -2.675132000 & 5.177064000 & -1.236280000 \\
\hline C & -1.695945000 & 4.742604000 & -2.137918000 \\
\hline C & -1.401921000 & 5.403095000 & -3.327142000 \\
\hline C & -2.097336000 & 6.569474000 & -3.636460000 \\
\hline H & -0.640197000 & 5.017405000 & -4.008666000 \\
\hline C & -3.061805000 & 7.044331000 & -2.752304000 \\
\hline H & -1.879889000 & 7.102782000 & -4.564178000 \\
\hline C & -3.345789000 & 6.358527000 & -1.572813000 \\
\hline H & -3.603504000 & 7.965388000 & -2.978145000 \\
\hline H & -4.094003000 & 6.758645000 & -0.888276000 \\
\hline I & -0.567850000 & 3.039635000 & -1.611666000 \\
\hline C & -4.347869000 & 2.270350000 & 0.316456000 \\
\hline H & -5.069340000 & 2.839765000 & 0.902464000 \\
\hline H & -5.431468000 & 0.429815000 & 0.551221000 \\
\hline C & -2.923270000 & 4.404162000 & 0.059651000 \\
\hline H & -1.949855000 & 4.402951000 & 0.589123000 \\
\hline B & 0.557104000 & 0.510401000 & 0.397136000 \\
\hline $\mathbf{F}$ & -0.259785000 & -0.584213000 & 0.591433000 \\
\hline $\mathbf{F}$ & 0.504345000 & 0.837029000 & -1.049787000 \\
\hline $\mathbf{F}$ & 0.039097000 & 1.649956000 & 1.035627000 \\
\hline $\mathbf{F}$ & 1.865386000 & 0.285593000 & 0.731804000 \\
\hline C & -3.913030000 & 5.067512000 & 0.986675000 \\
\hline C & -5.255312000 & 5.250389000 & 0.623319000 \\
\hline C & -3.495714000 & 5.506209000 & 2.245102000 \\
\hline C & -6.151435000 & 5.860070000 & 1.497071000 \\
\hline C & -4.391306000 & 6.117067000 & 3.122447000 \\
\hline C & -5.720915000 & 6.296546000 & 2.750343000 \\
\hline H & -5.604525000 & 4.907627000 & -0.354371000 \\
\hline H & -2.454402000 & 5.362020000 & 2.545584000 \\
\hline H & -7.193857000 & 5.993379000 & 1.198397000 \\
\hline H & -4.045342000 & 6.451220000 & 4.103134000 \\
\hline H & -6.424365000 & 6.773724000 & 3.436461000 \\
\hline
\end{tabular}

Coordinates of \#15 R = Ph (8d'), transition state conformer

39

$\begin{array}{llll}\text { symmetry c1 } & & & \\ \text { C } & -2.808614000 & 1.500779000 & -1.092620000 \\ \text { C } & -2.282512000 & 2.747434000 & -1.448871000 \\ \text { H } & -2.570280000 & 0.607875000 & -1.677624000 \\ \text { C } & -3.939424000 & 2.542813000 & 0.749040000 \\ \text { H } & -4.052570000 & 0.425397000 & 0.294211000 \\ \text { C } & -2.541913000 & 3.907997000 & -0.727312000 \\ \text { C } & -3.641014000 & 1.396603000 & 0.012406000 \\ \text { C } & -1.034962000 & 5.581731000 & -2.134716000 \\ \text { C } & -0.516105000 & 4.720927000 & -3.101651000 \\ \text { C } & 0.411134000 & 5.096858000 & -4.072538000 \\ \text { C } & 0.836699000 & 6.419099000 & -4.105837000 \\ \text { H } & 0.805984000 & 4.386444000 & -4.800502000 \\ \text { C } & 0.328582000 & 7.330666000 & -3.184207000\end{array}$




$\begin{array}{lrll}\text { H } & 1.565973000 & 6.719496000 & -4.860435000 \\ \mathbf{C} & -0.578119000 & 6.910110000 & -2.222931000 \\ \mathbf{H} & 0.647286000 & 8.374864000 & -3.204256000 \\ \mathbf{H} & -0.947604000 & 7.625703000 & -1.485292000 \\ \mathbf{I} & -1.141725000 & 2.695398000 & -3.215992000 \\ \mathbf{C} & -3.392363000 & 3.763991000 & 0.384539000 \\ \mathbf{H} & -3.612187000 & 4.649387000 & 0.984908000 \\ \mathbf{H} & -4.596040000 & 2.483005000 & 1.619364000 \\ \mathbf{C} & -2.013098000 & 5.308122000 & -0.997372000 \\ \mathbf{H} & -2.904922000 & 5.916399000 & -1.230153000 \\ \mathbf{B} & 1.305947000 & 2.055901000 & -5.585377000 \\ \mathbf{F} & -0.096225000 & 2.511902000 & -5.536375000 \\ \mathbf{F} & 2.054876000 & 3.139414000 & -6.023117000 \\ \mathbf{F} & 1.624143000 & 1.743595000 & -4.255581000 \\ \mathbf{F} & 1.384143000 & 0.968787000 & -6.410616000 \\ \mathbf{C} & -1.430592000 & 5.863346000 & 0.297003000 \\ \mathbf{C} & -2.046974000 & 6.920165000 & 0.968986000 \\ \mathbf{C} & -0.263744000 & 5.305446000 & 0.829791000 \\ \mathbf{C} & -1.508556000 & 7.414797000 & 2.158172000 \\ \mathbf{C} & 0.274296000 & 5.797126000 & 2.014291000 \\ \mathbf{C} & -0.347594000 & 6.853655000 & 2.682698000 \\ \mathbf{H} & -2.958489000 & 7.364486000 & 0.557896000 \\ \mathbf{H} & 0.228637000 & 4.480403000 & 0.307634000 \\ \mathbf{H} & -2.000126000 & 8.243136000 & 2.673613000 \\ \mathbf{H} & 1.187000000 & 5.354482000 & 2.419720000 \\ \mathbf{H} & 0.076625000 & 7.239274000 & 3.612490000\end{array}$


6 NMR-Spectra 


\subsection{NMR Spectra of New Substrates}

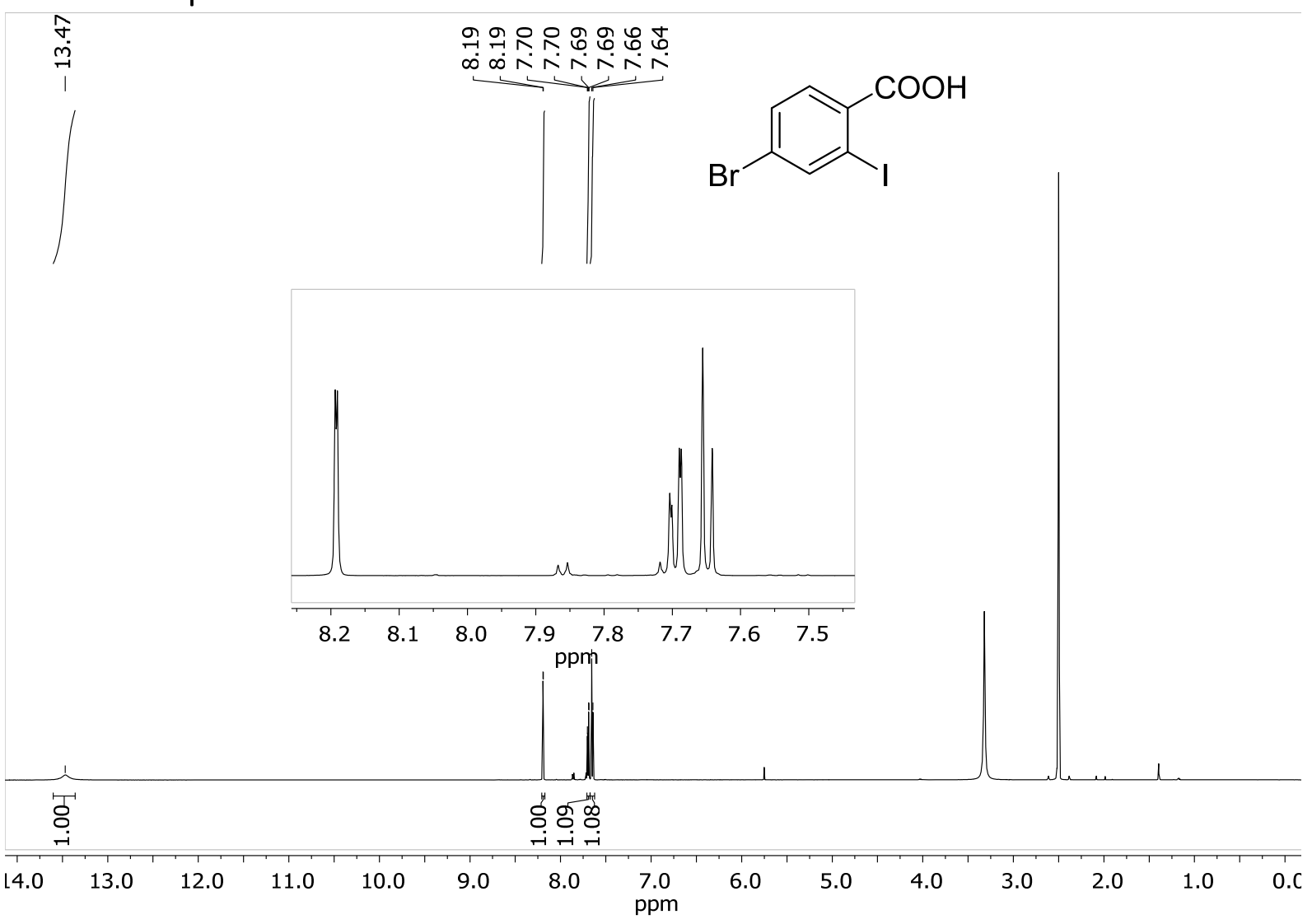

Figure S2: $600 \mathrm{MHz}{ }^{1} \mathrm{H}-\mathrm{NMR}$ spectrum of compound $\boldsymbol{S 1 h}$.

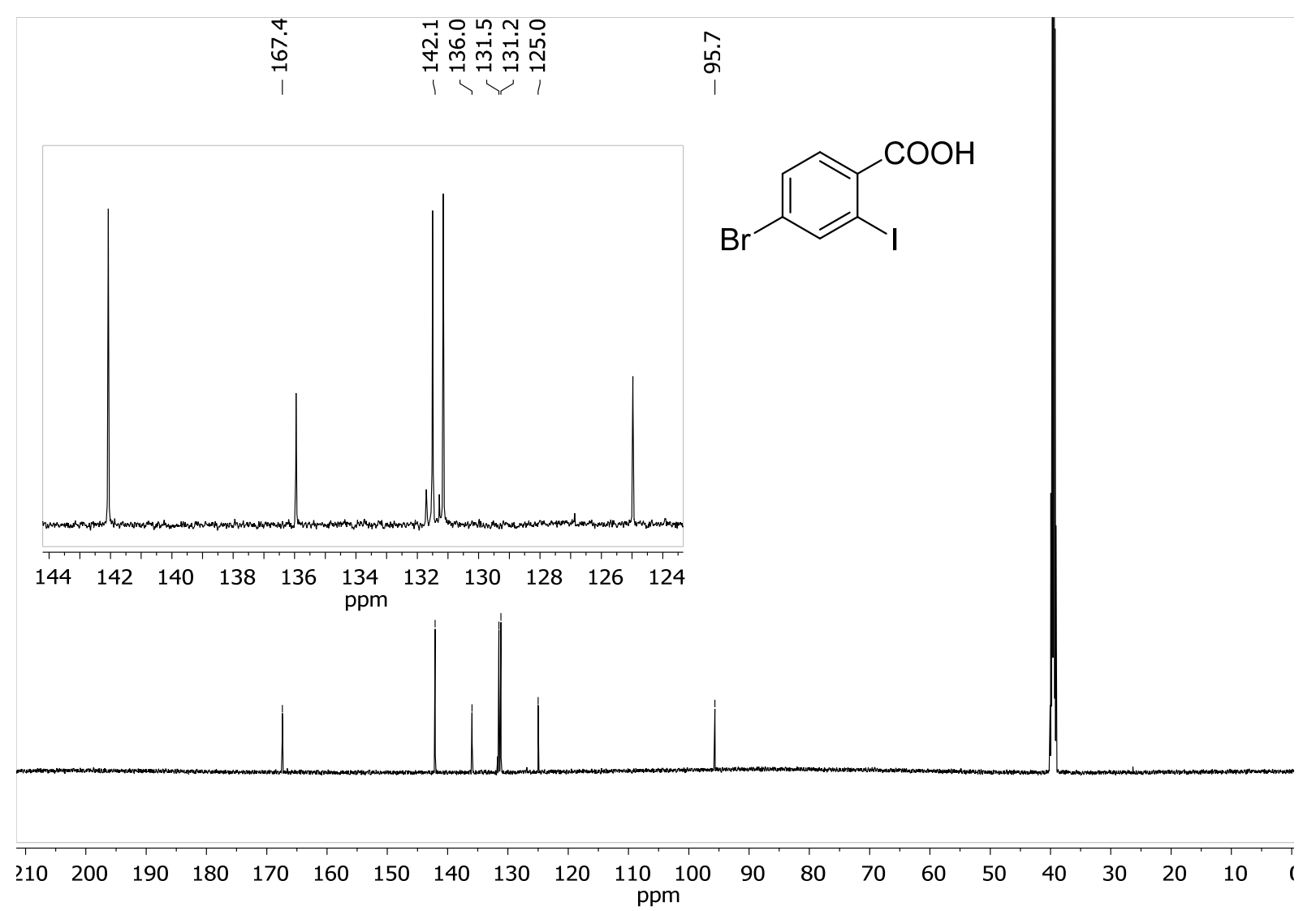

Figure S3: $151 \mathrm{MHz}{ }^{13} \mathrm{C}-\mathrm{NMR}$ spectrum of compound S1h. 


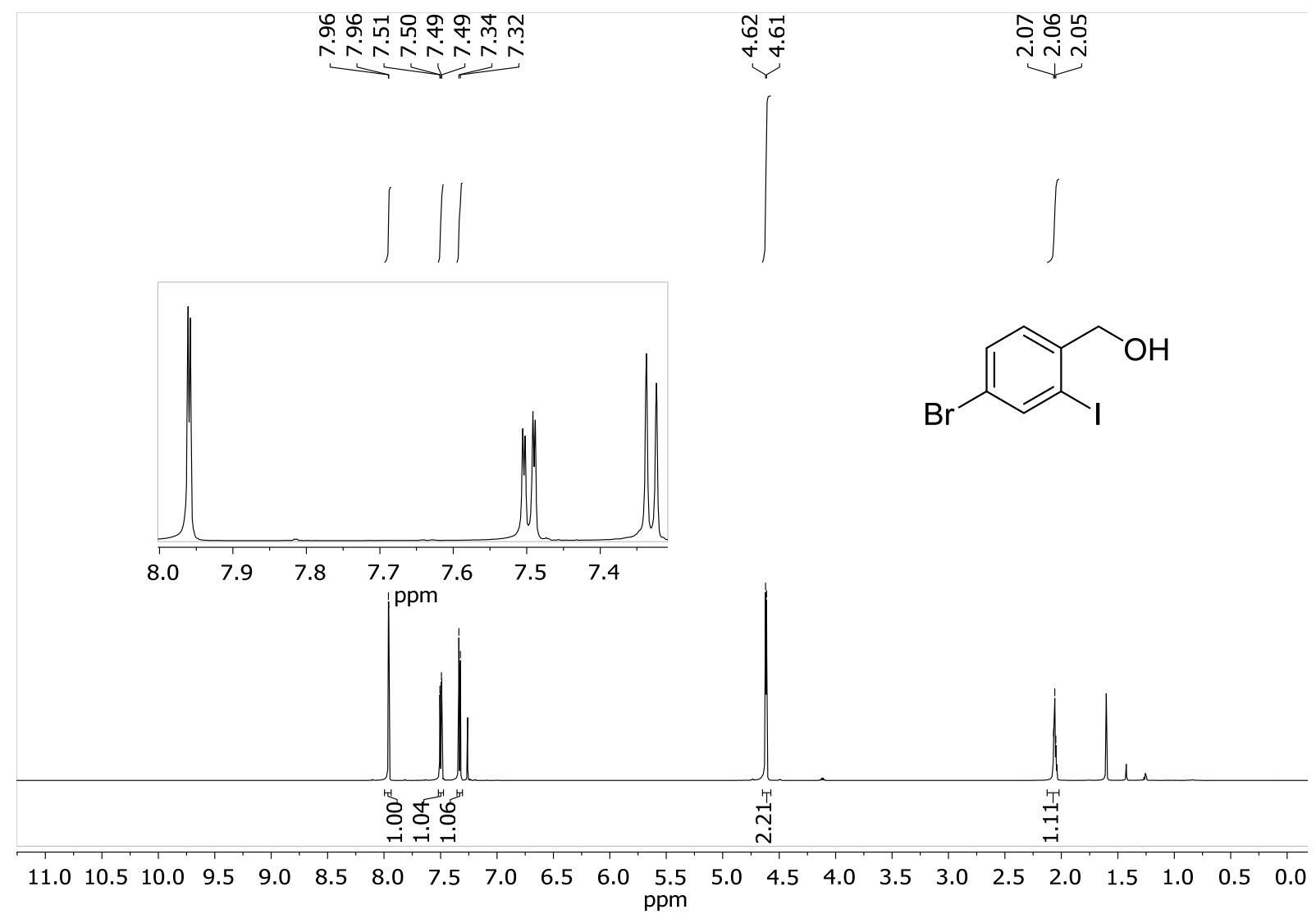

Figure S4: $600 \mathrm{MHz}{ }^{1} \mathrm{H}-\mathrm{NMR}$ spectrum of compound $\mathbf{5 h}$.
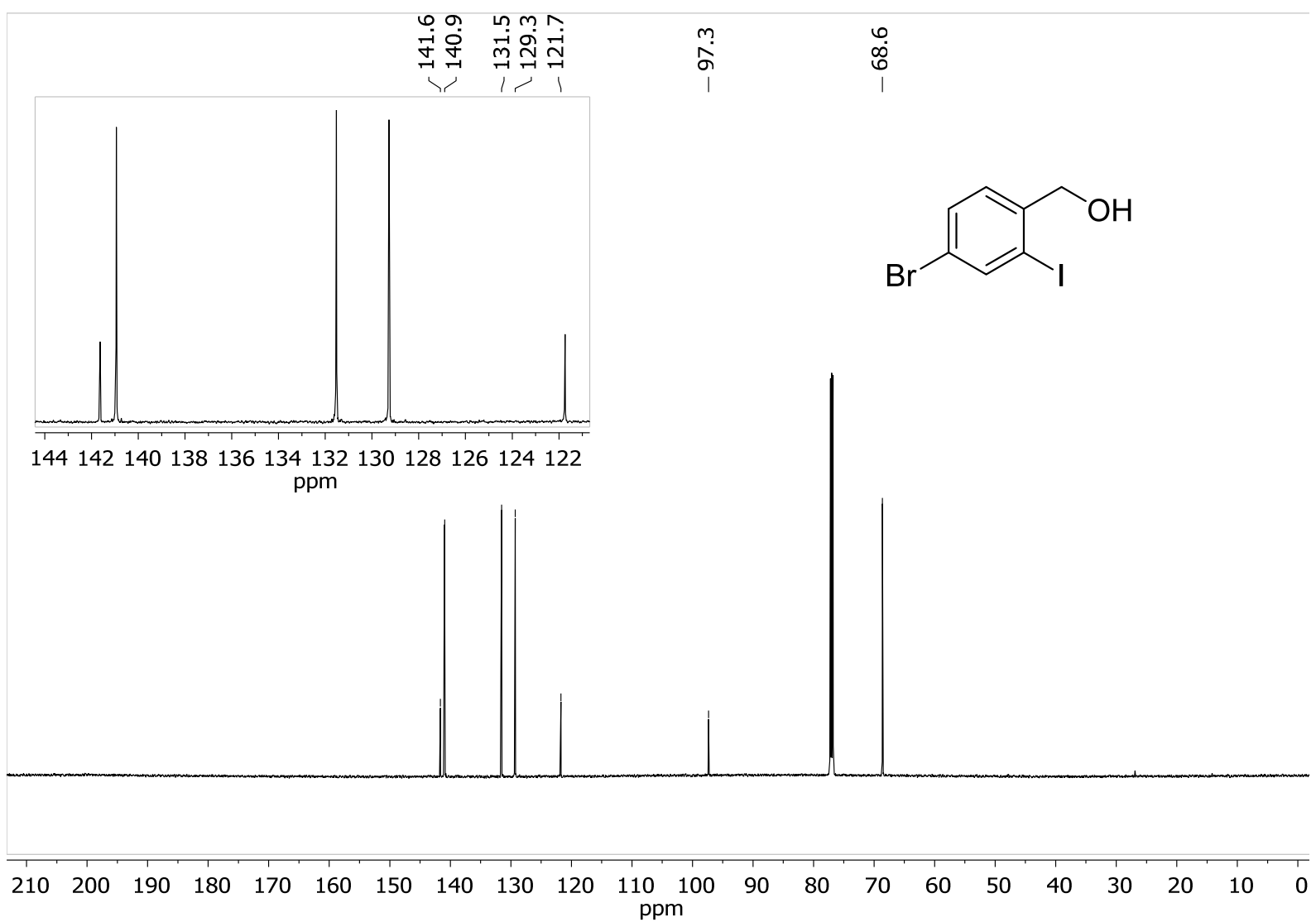

Figure S5: $151 \mathrm{MHz}{ }^{13} \mathrm{C}-\mathrm{NMR}$ spectrum of compound $\mathbf{5 h}$. 


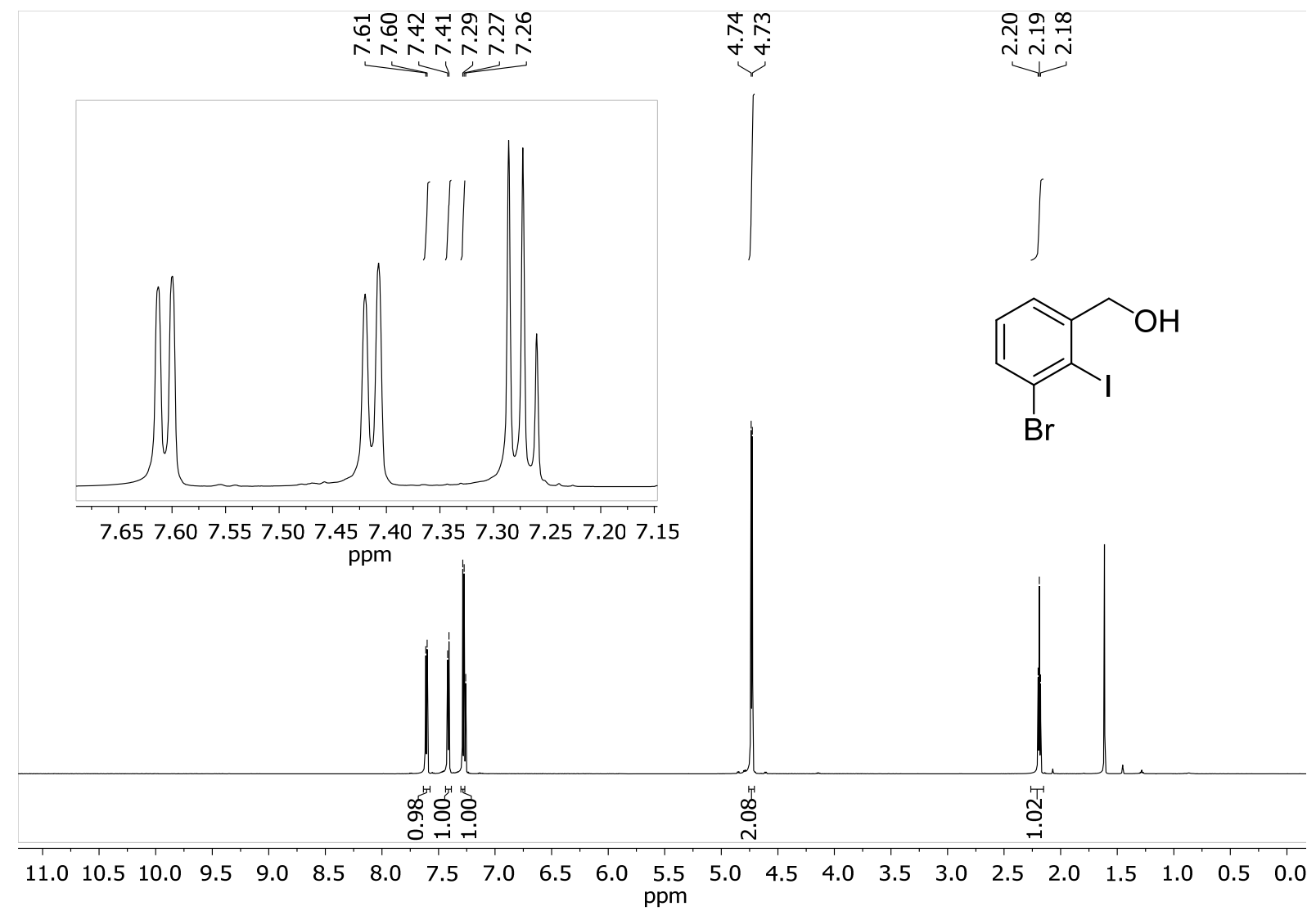

Figure S6: $600 \mathrm{MHz}{ }^{1} \mathrm{H}-\mathrm{NMR}$ spectrum of compound $\mathbf{5 i}$.
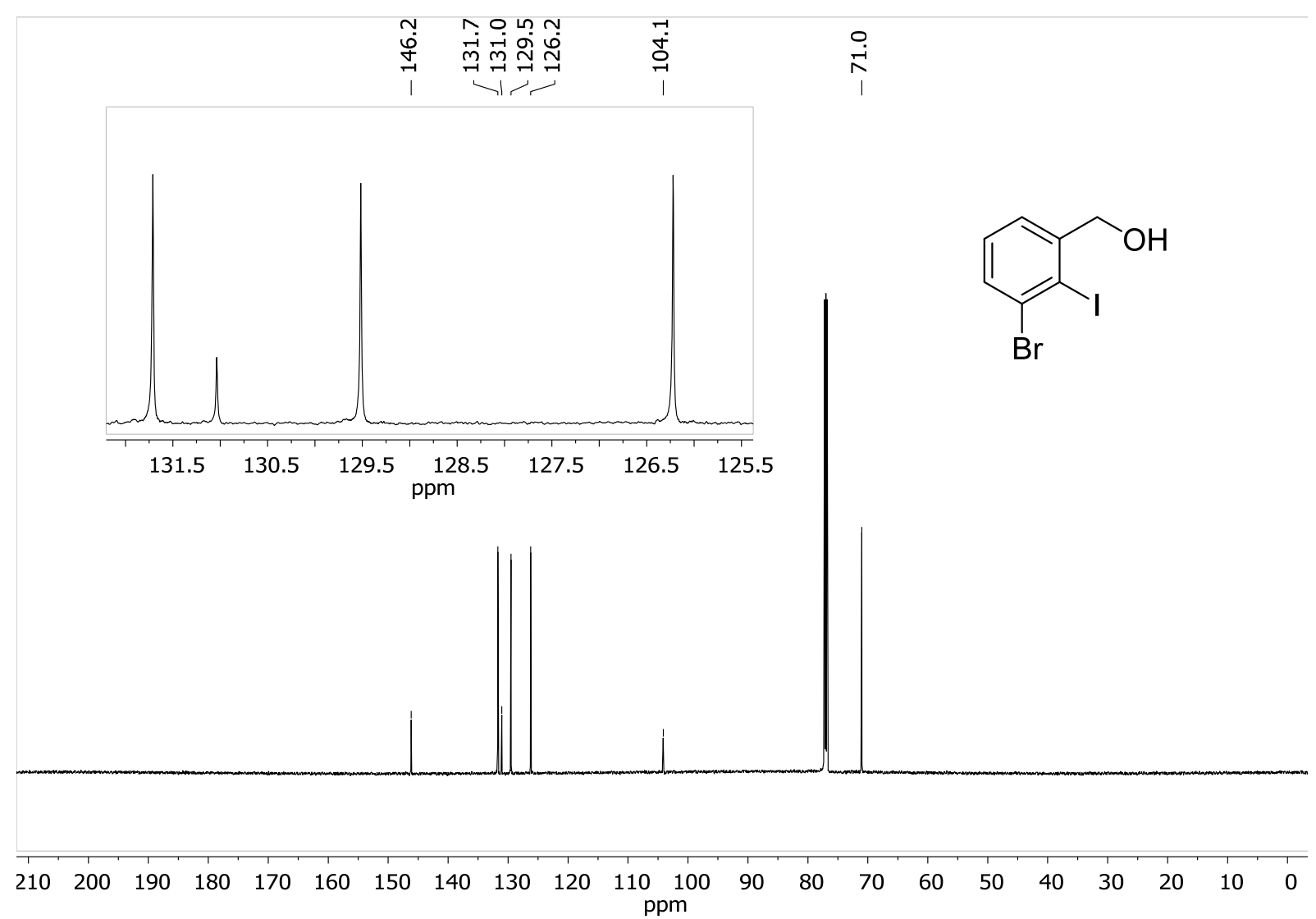

Figure S7: $151 \mathrm{MHz}{ }^{13} \mathrm{C}-\mathrm{NMR}$ spectrum of compound $\mathbf{5 i}$. 


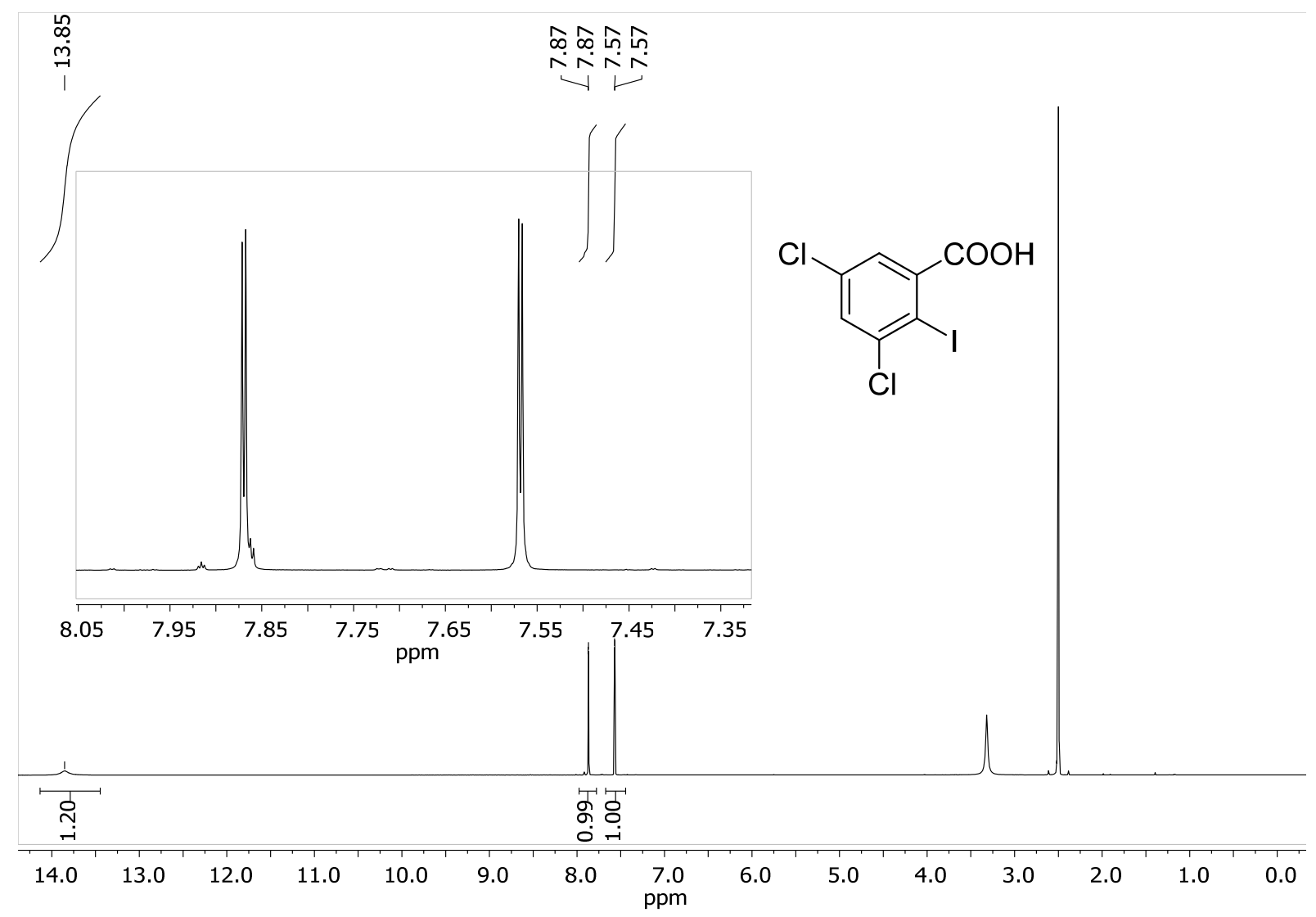

Figure S8: $600 \mathrm{MHz}{ }^{1} \mathrm{H}-\mathrm{NMR}$ spectrum of compound $\mathbf{S 1 j}$.

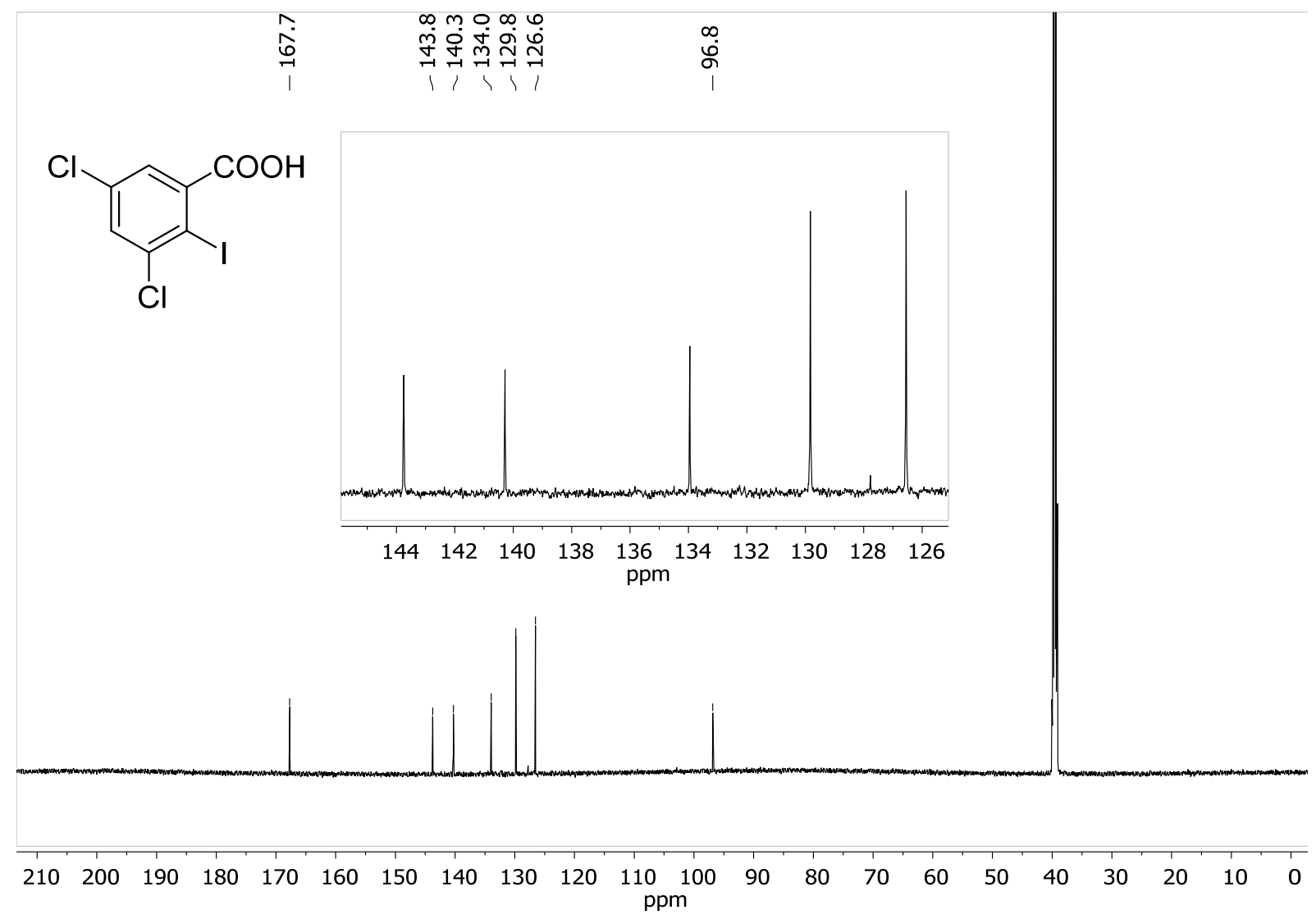

Figure S9: $151 \mathrm{MHz}{ }^{13} \mathrm{C}-N M R$ spectrum of compound $\mathbf{S 1 j}$. 


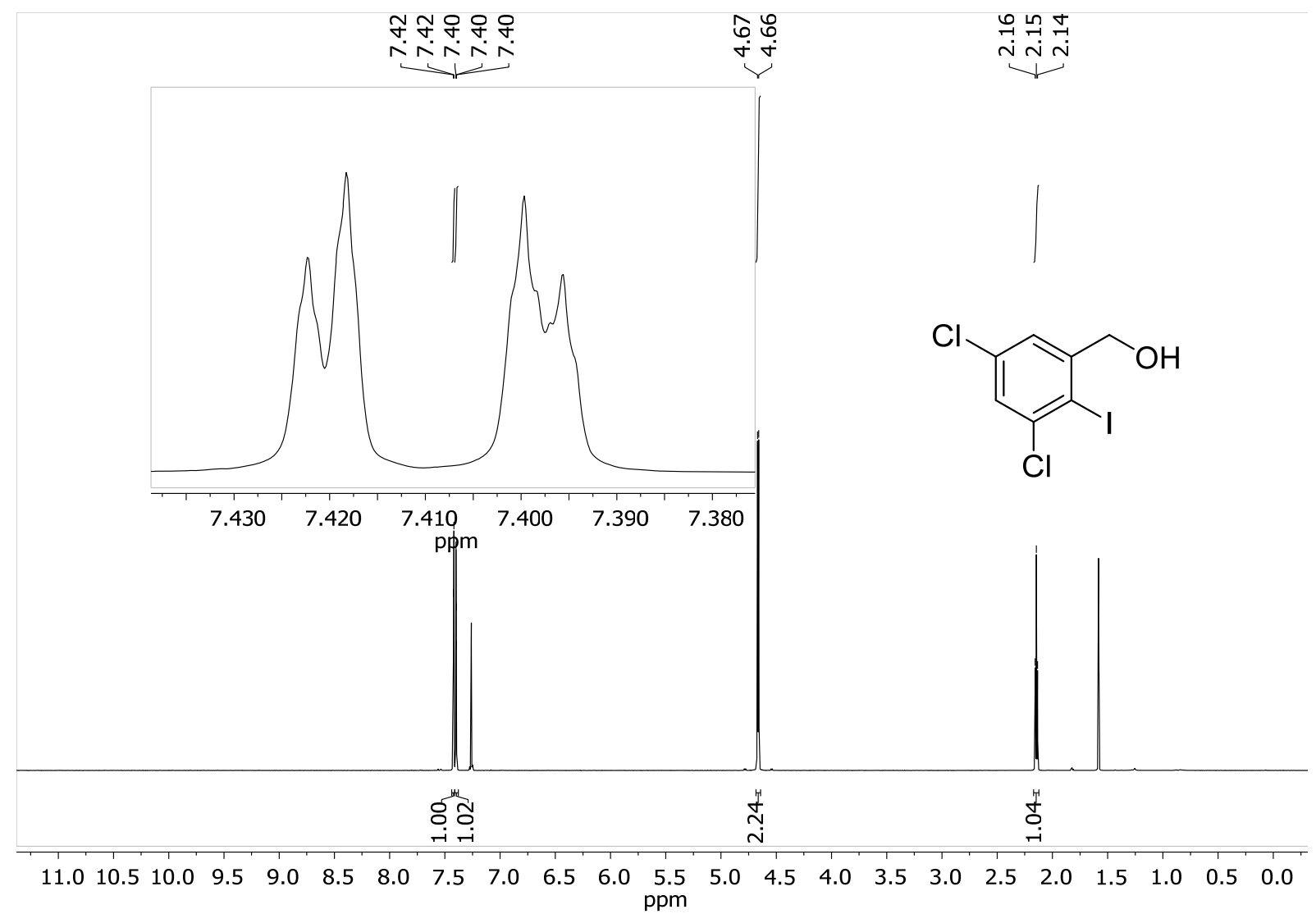

Figure S10: $600 \mathrm{MHz}{ }^{1} \mathrm{H}-\mathrm{NMR}$ spectrum of compound $\mathbf{5 j}$.

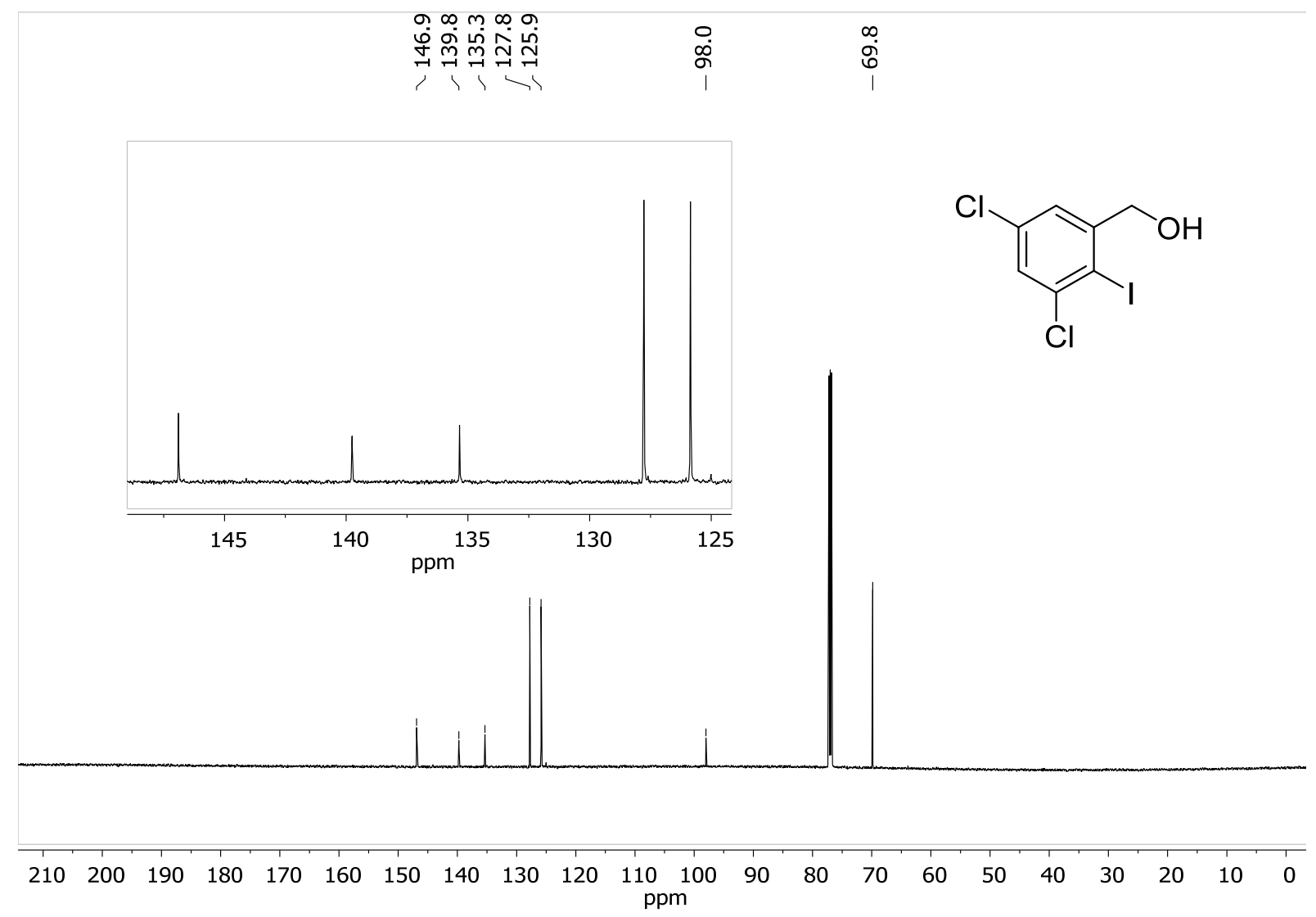

Figure S11: $151 \mathrm{MHz}{ }^{13} \mathrm{C}-N M R$ spectrum of compound $\mathbf{5 j}$. 


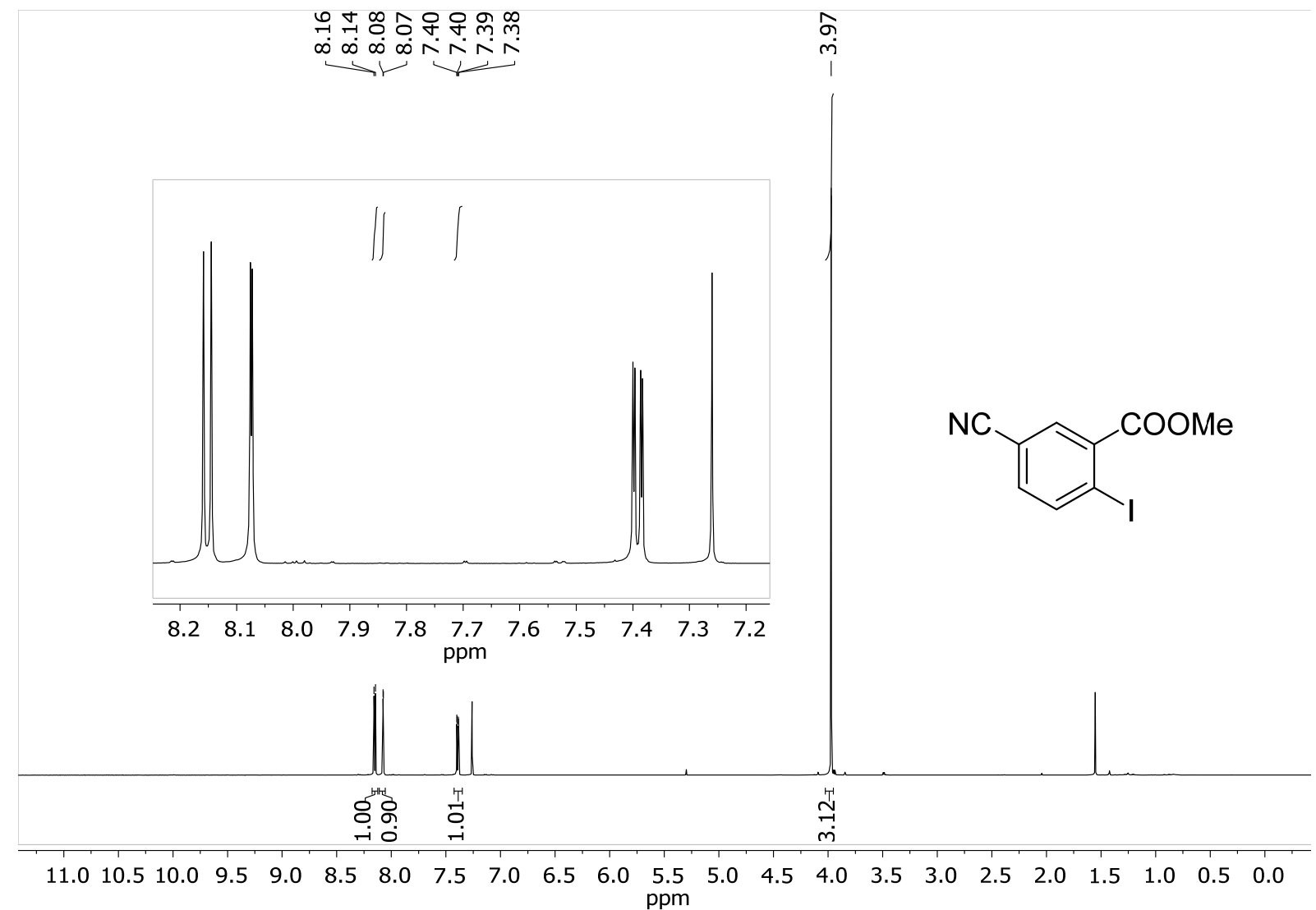

Figure S12: $600 \mathrm{MHz}{ }^{1} \mathrm{H}-\mathrm{NMR}$ spectrum of methyl 5-cyano-2-iodobenzoate.

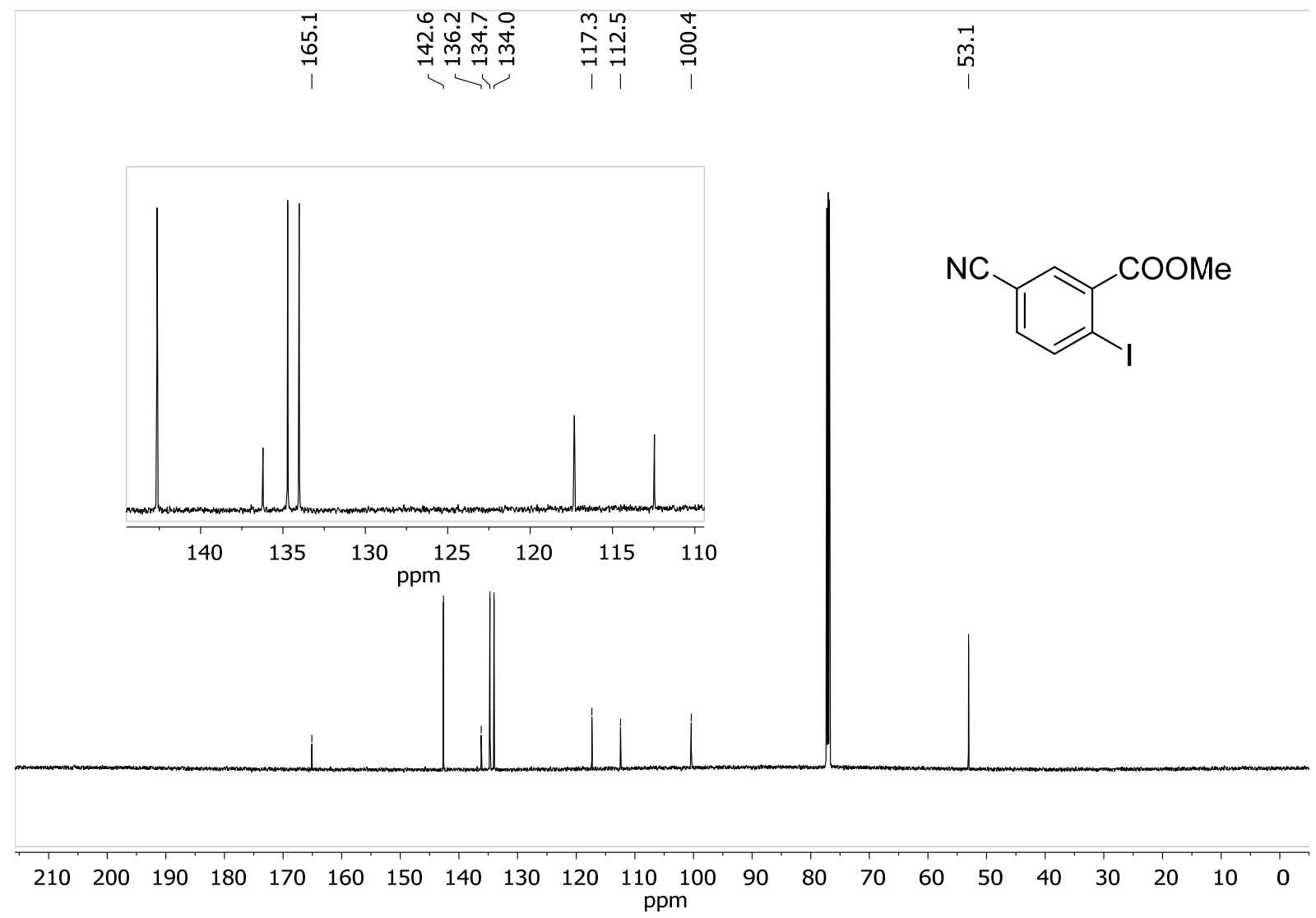

Figure S13: $151 \mathrm{MHz}{ }^{13} \mathrm{C}-\mathrm{NMR}$ spectrum of methyl 5-cyano-2-iodobenzoate. 


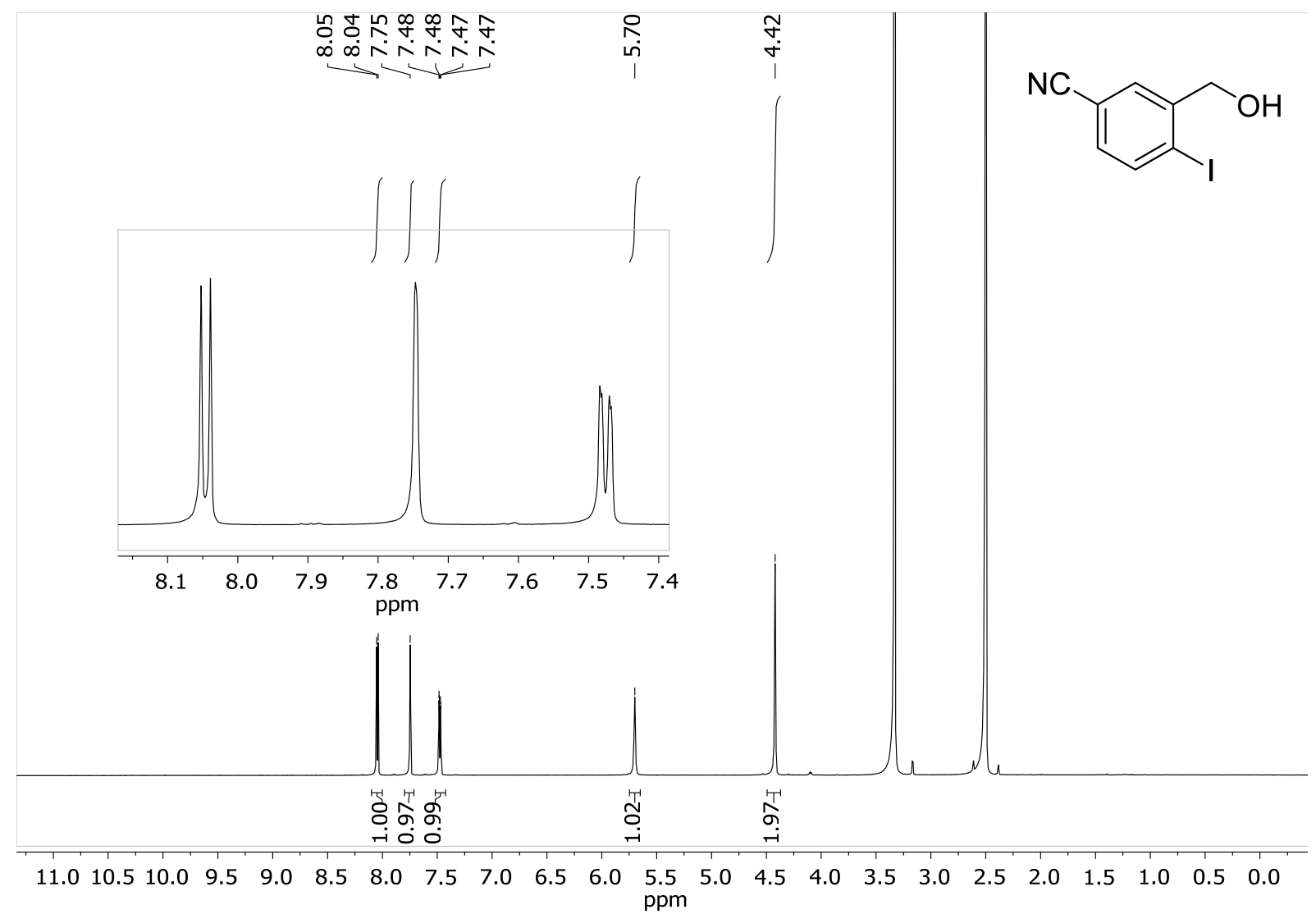

Figure S14: $600 \mathrm{MHz}{ }^{1} \mathrm{H}-\mathrm{NMR}$ spectrum of compound $\mathbf{5 g}$.

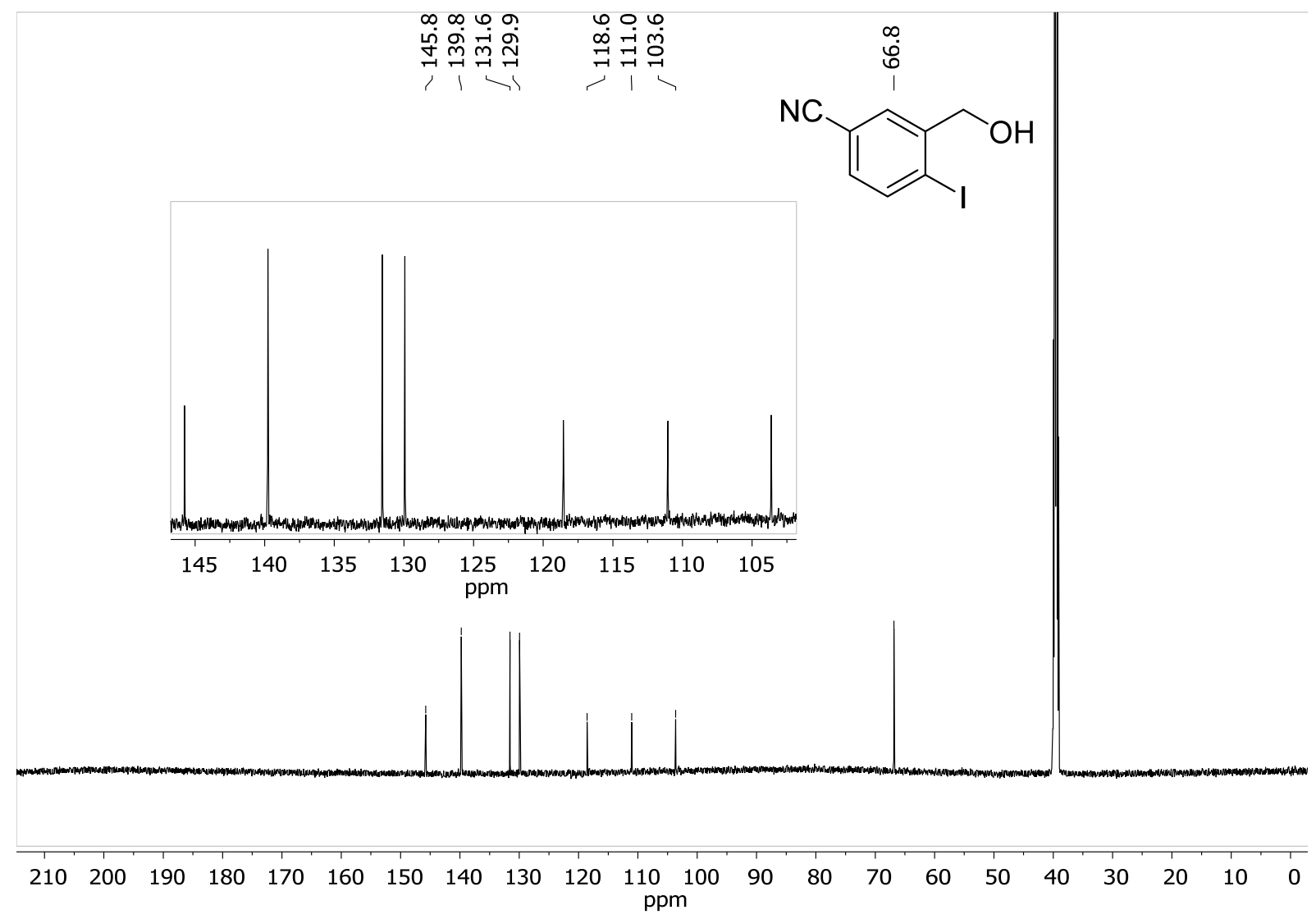

Figure S15: $151 \mathrm{MHz}{ }^{13} \mathrm{C}-\mathrm{NMR}$ spectrum of compound $\mathbf{5 g}$. 


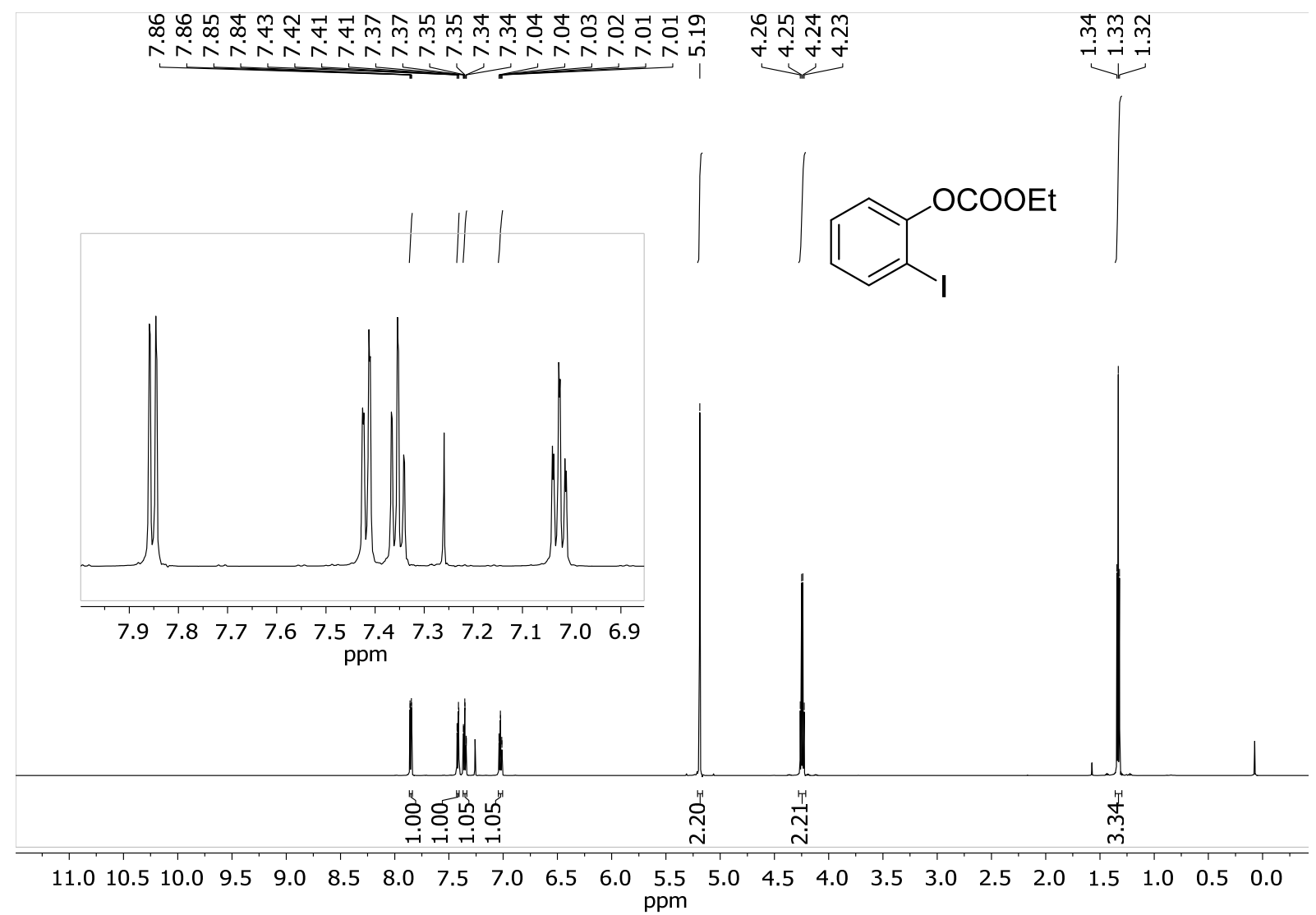

Figure S16: $600 \mathrm{MHz}{ }^{1} \mathrm{H}-\mathrm{NMR}$ spectrum of compound 12.

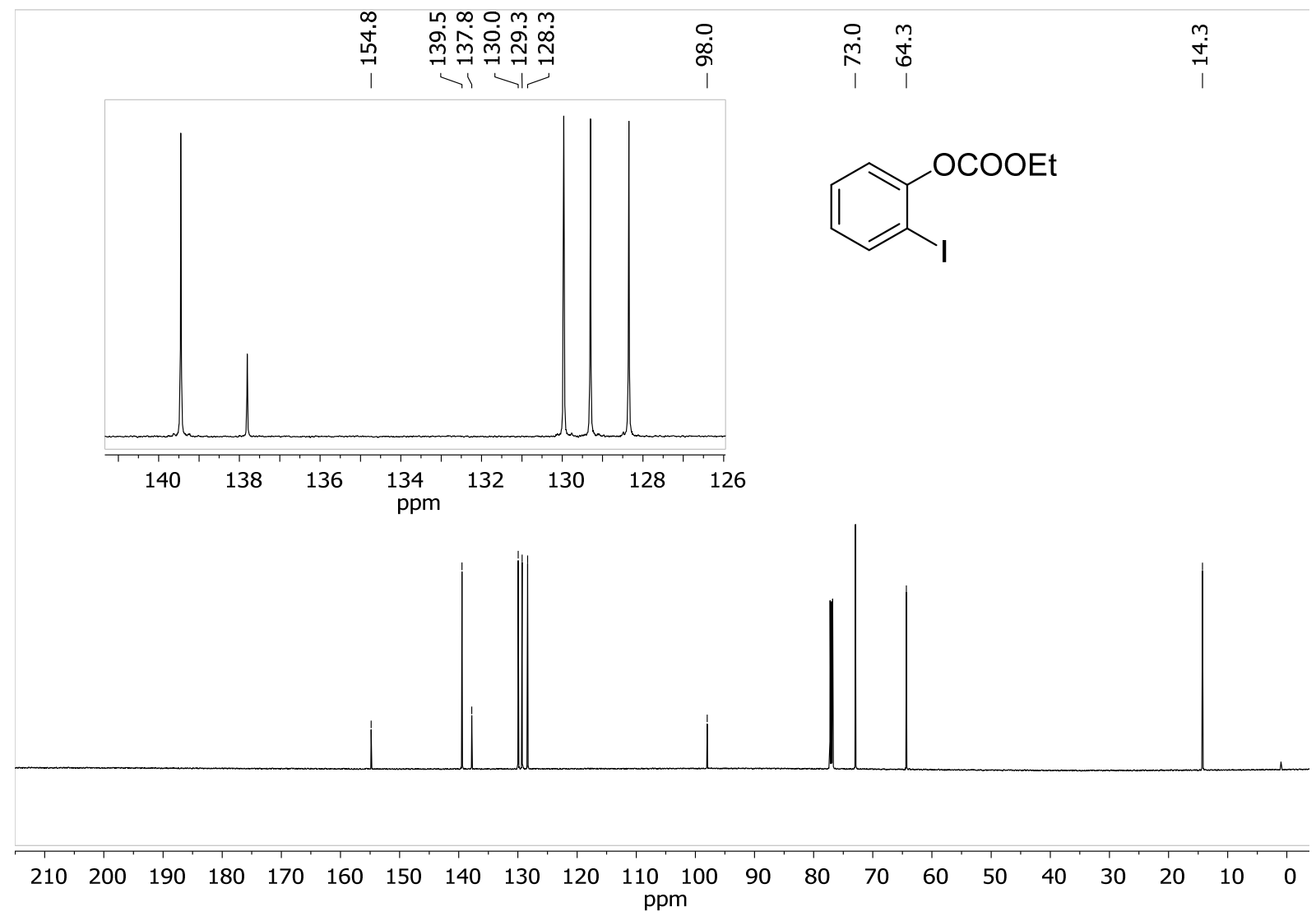

Figure S17: $151 \mathrm{MHz}{ }^{13} \mathrm{C}-\mathrm{NMR}$ spectrum of compound 12. 

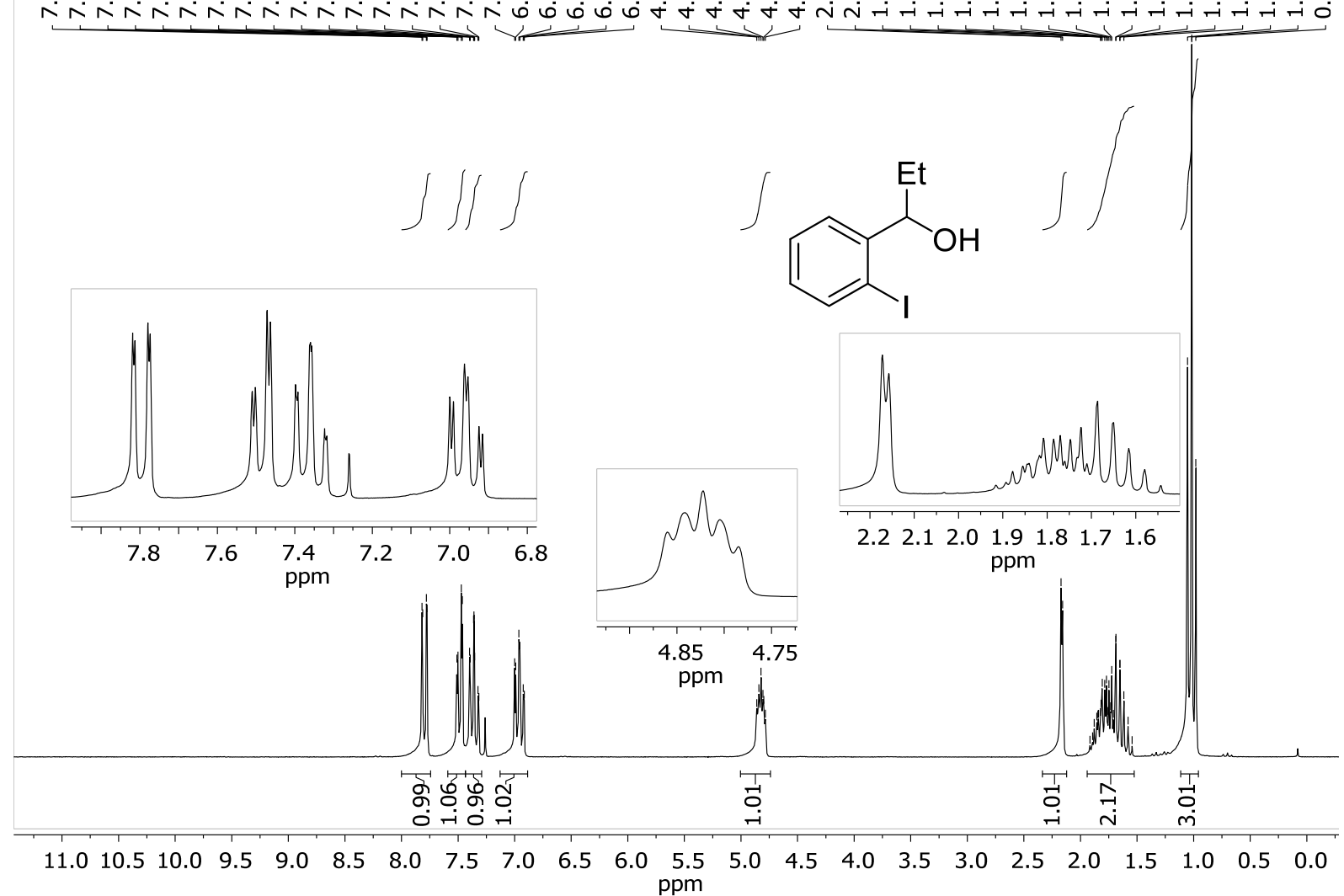

Figure S18: $200 \mathrm{MHz}{ }^{1} \mathrm{H}-\mathrm{NMR}$ spectrum of compound $\mathbf{5 0 .}$

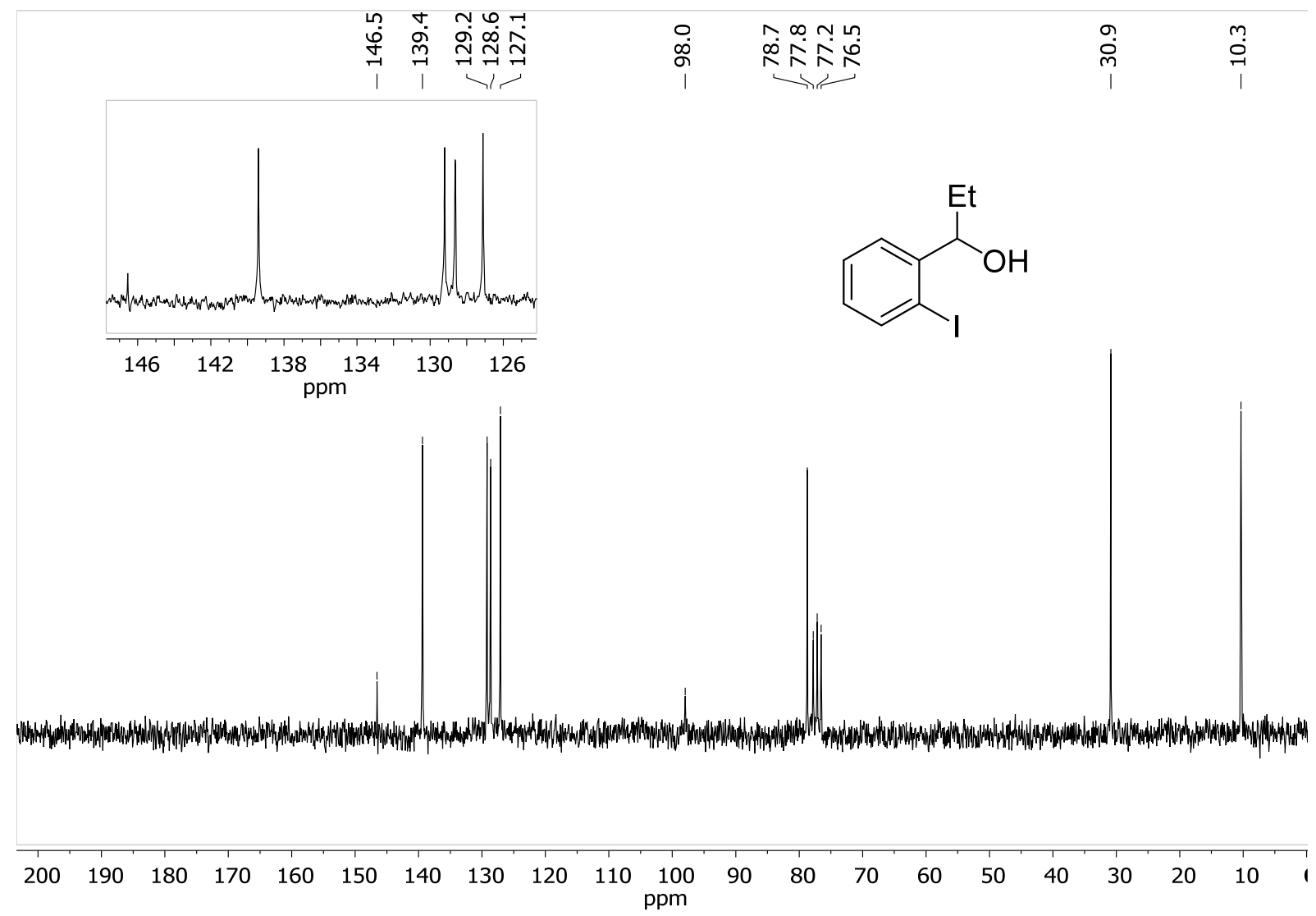

Figure S19: $50 \mathrm{MHz}{ }^{13} \mathrm{C}-\mathrm{NMR}$ spectrum of compound $\mathbf{5 0}$. 


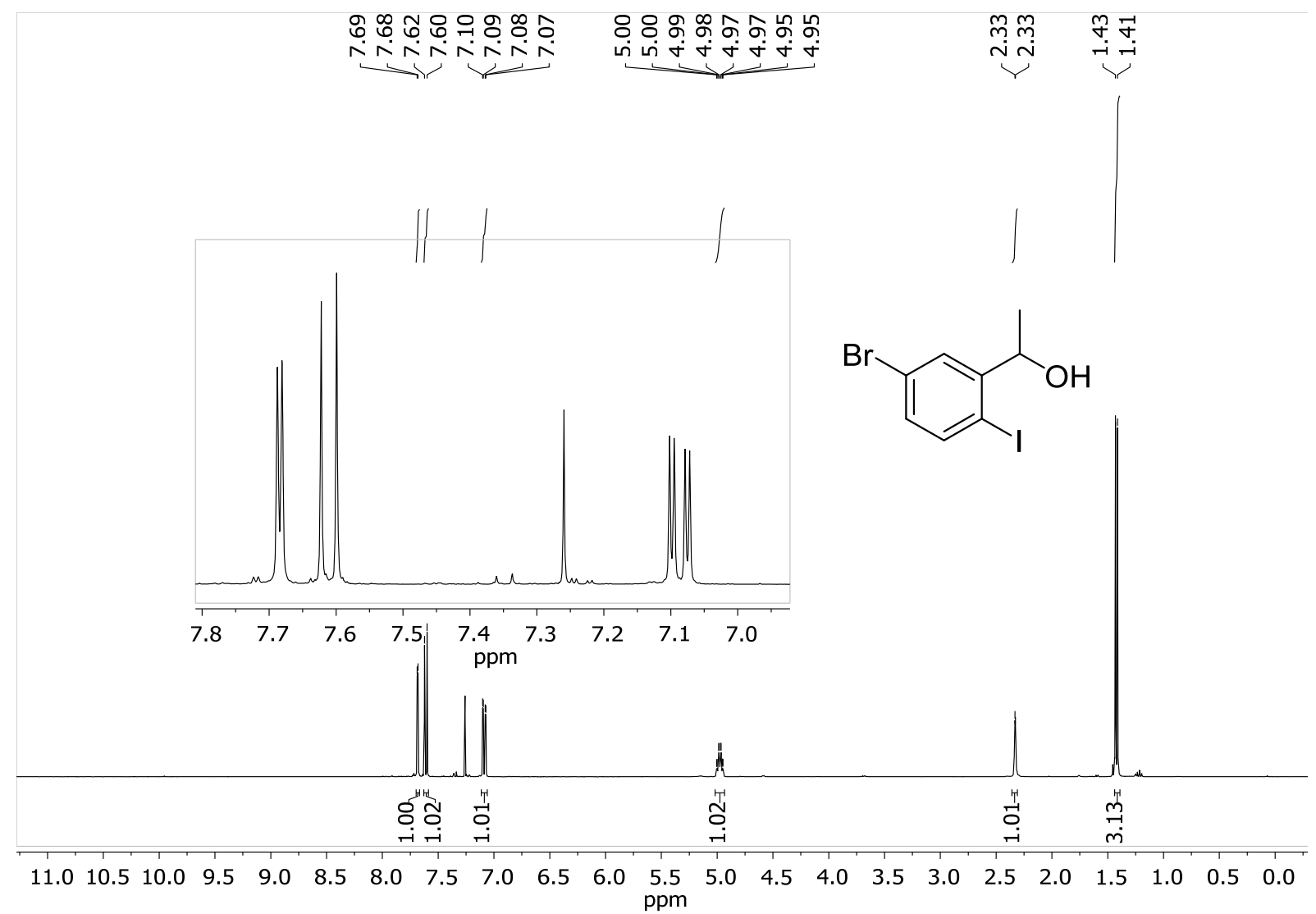

Figure S20: $360 \mathrm{MHz}{ }^{1} \mathrm{H}-\mathrm{NMR}$ spectrum of compound $\mathbf{5 q}$.

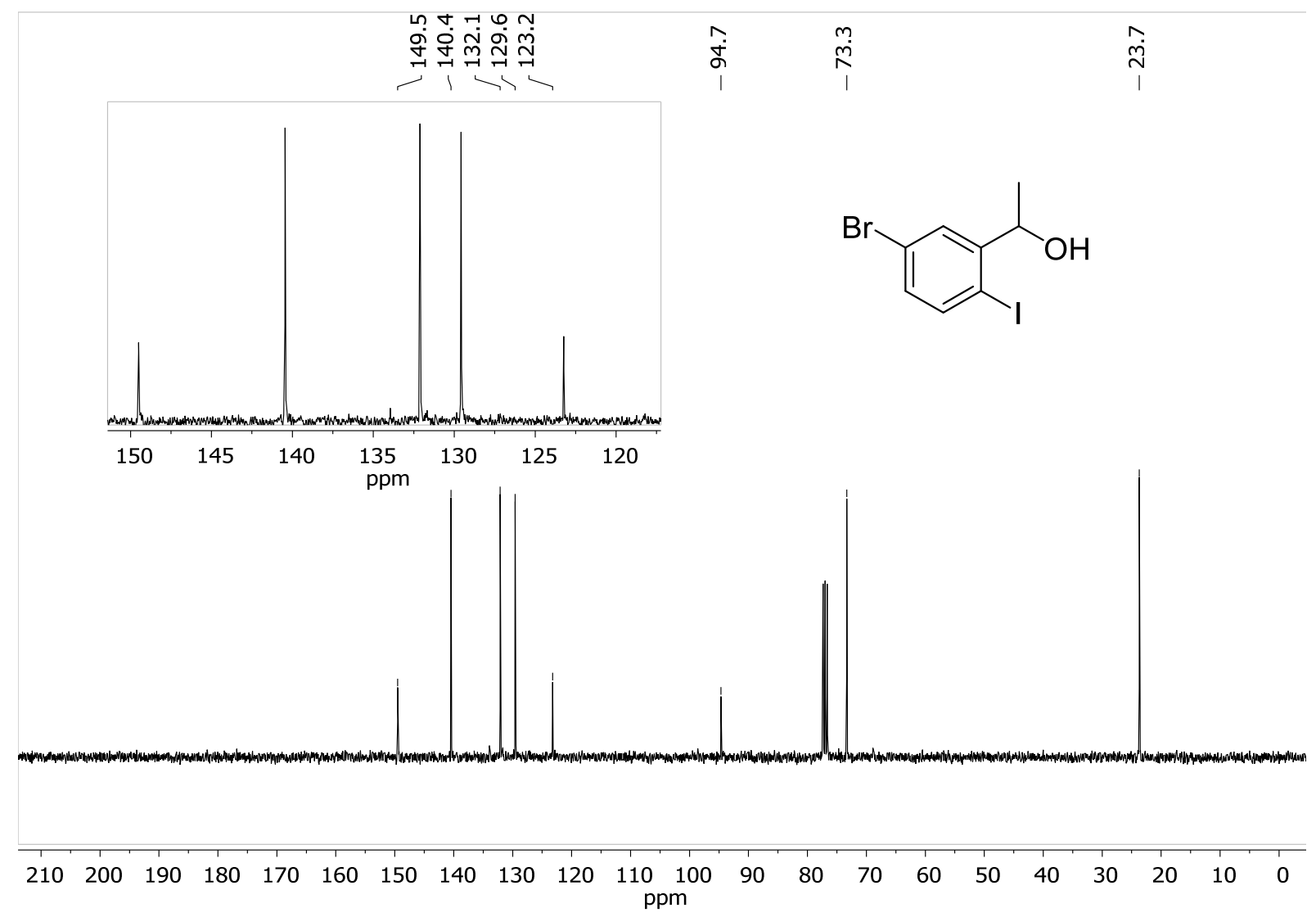

Figure S21: $90 \mathrm{MHz}{ }^{13} \mathrm{C}-\mathrm{NMR}$ spectrum of compound $\mathbf{5 q}$. 


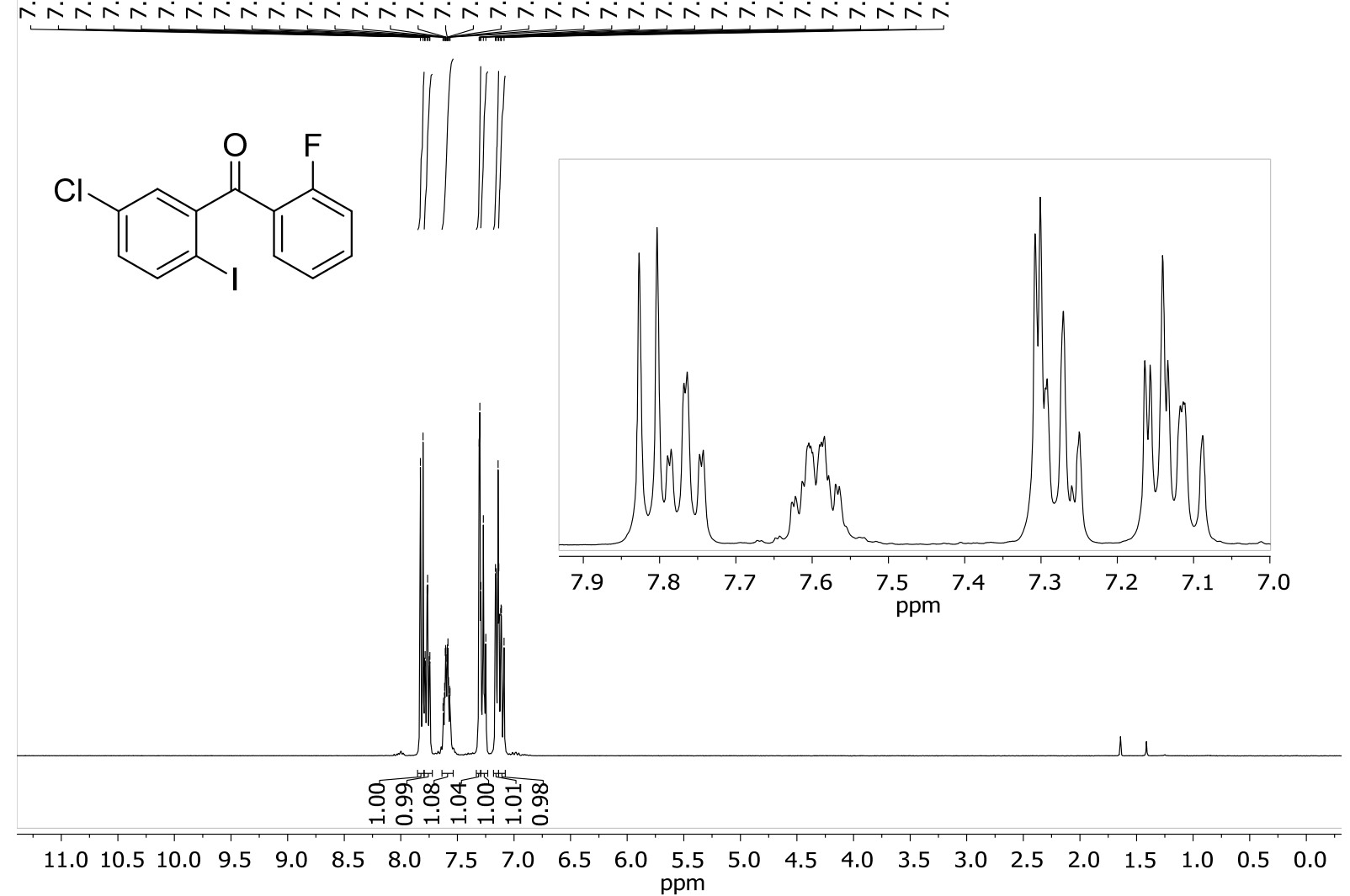

Figure S22: $360 \mathrm{MHz}{ }^{1} \mathrm{H}-\mathrm{NMR}$ spectrum of compound (5-chloro-2-iodophenyl)(2-fluorophenyl)methanone.
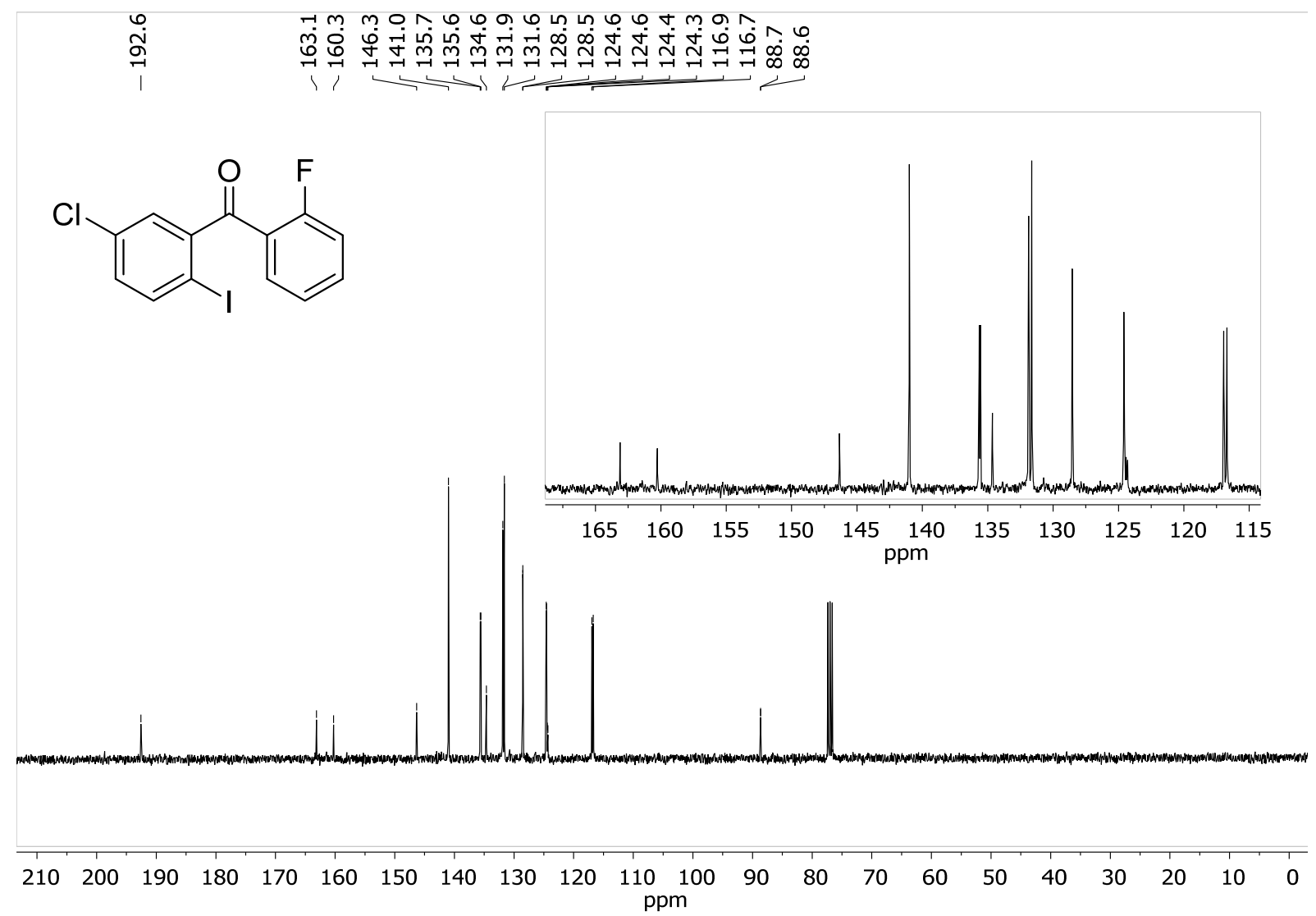

Figure S23: $90 \mathrm{MHz}{ }^{13} \mathrm{C}-\mathrm{NMR}$ spectrum of compound (5-chloro-2-iodophenyl)(2-fluorophenyl)methanone. 


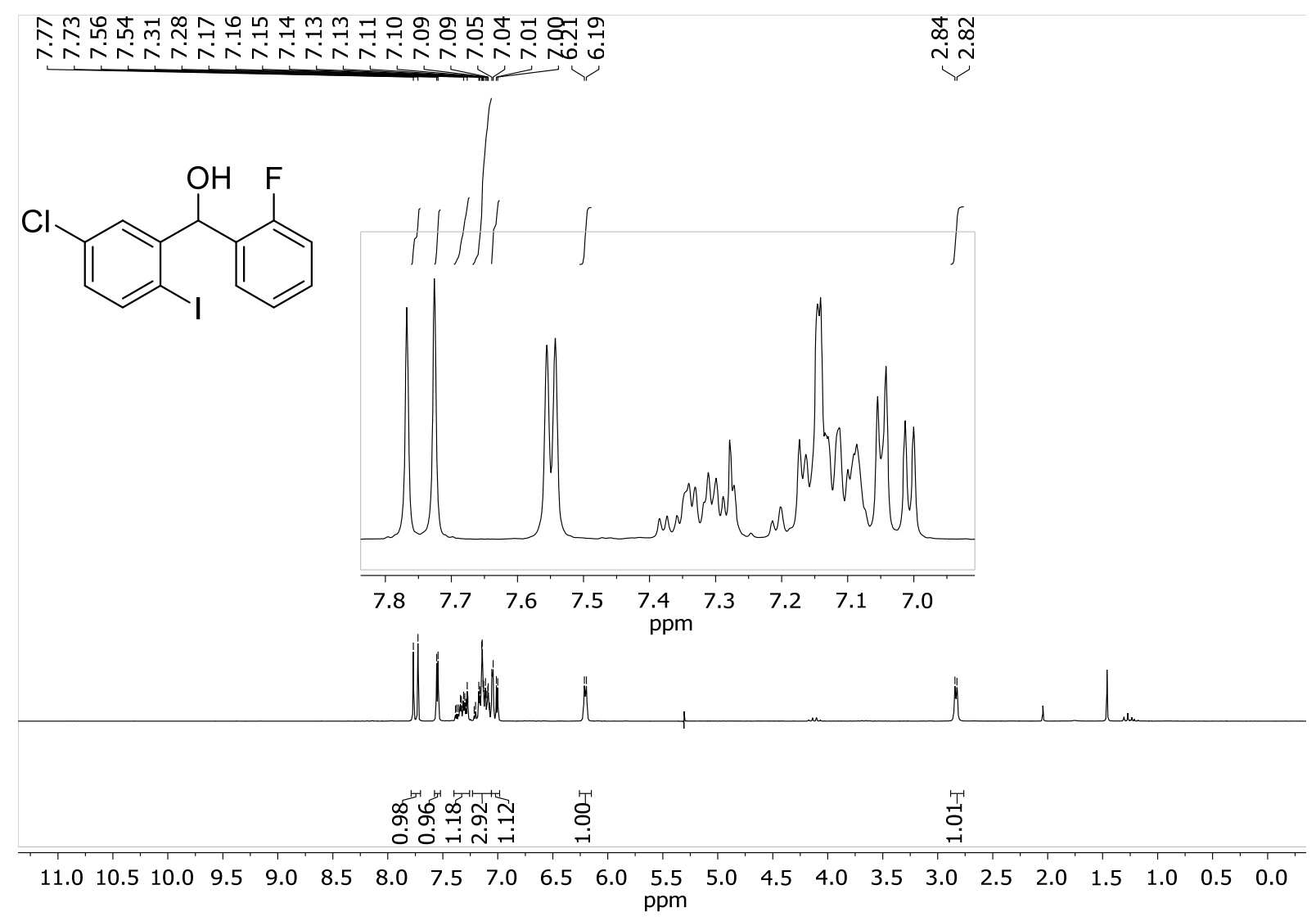

Figure S24: $200 \mathrm{MHz}{ }^{1} \mathrm{H}-\mathrm{NMR}$ spectrum of compound $\mathbf{5 m}$.
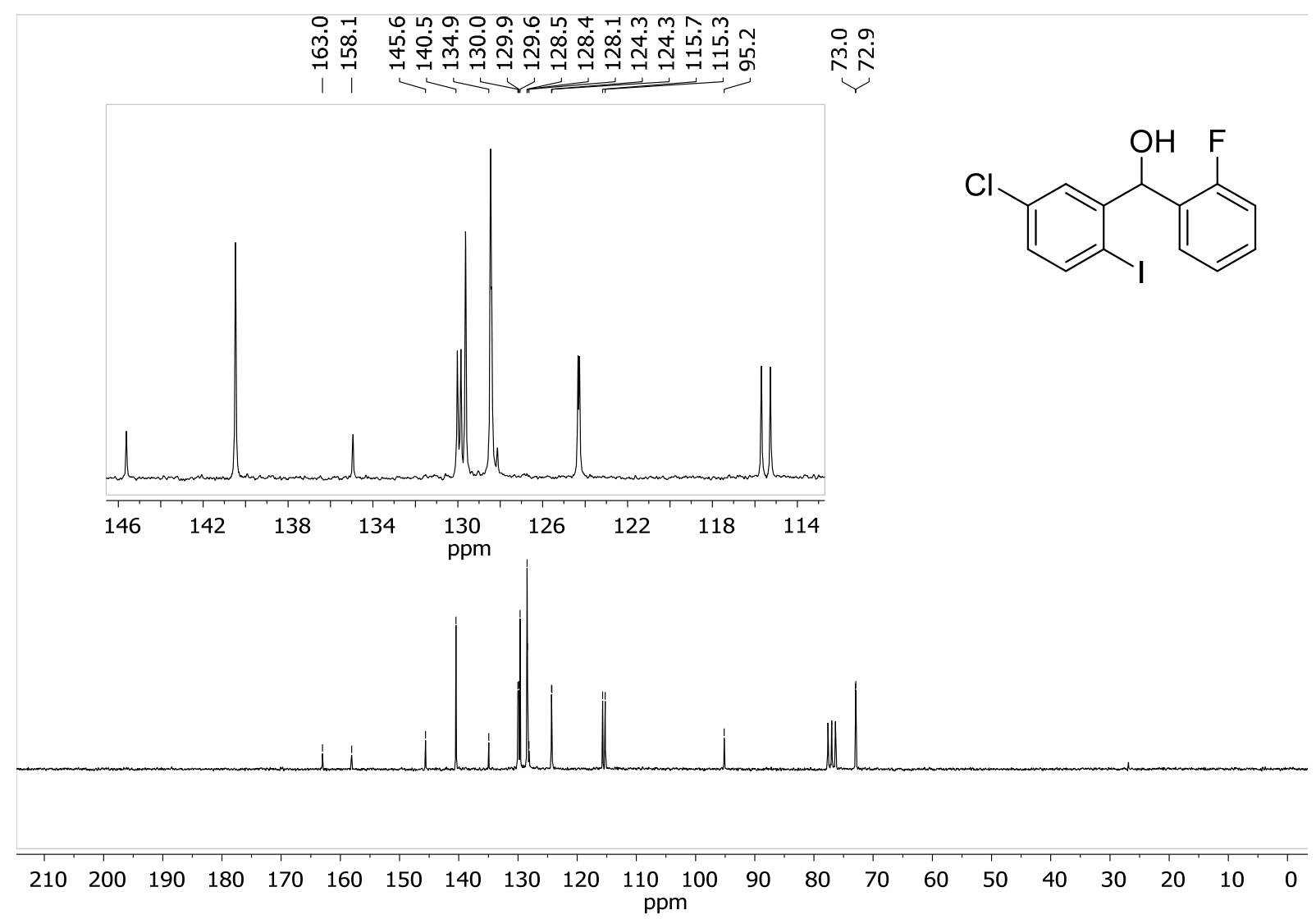

Figure S25: $50 \mathrm{MHz}{ }^{13} \mathrm{C}-\mathrm{NMR}$ spectrum of compound $5 \mathrm{~m}$. 
6.3 NMR-Spetra of lodoniumsalts 7 and 8

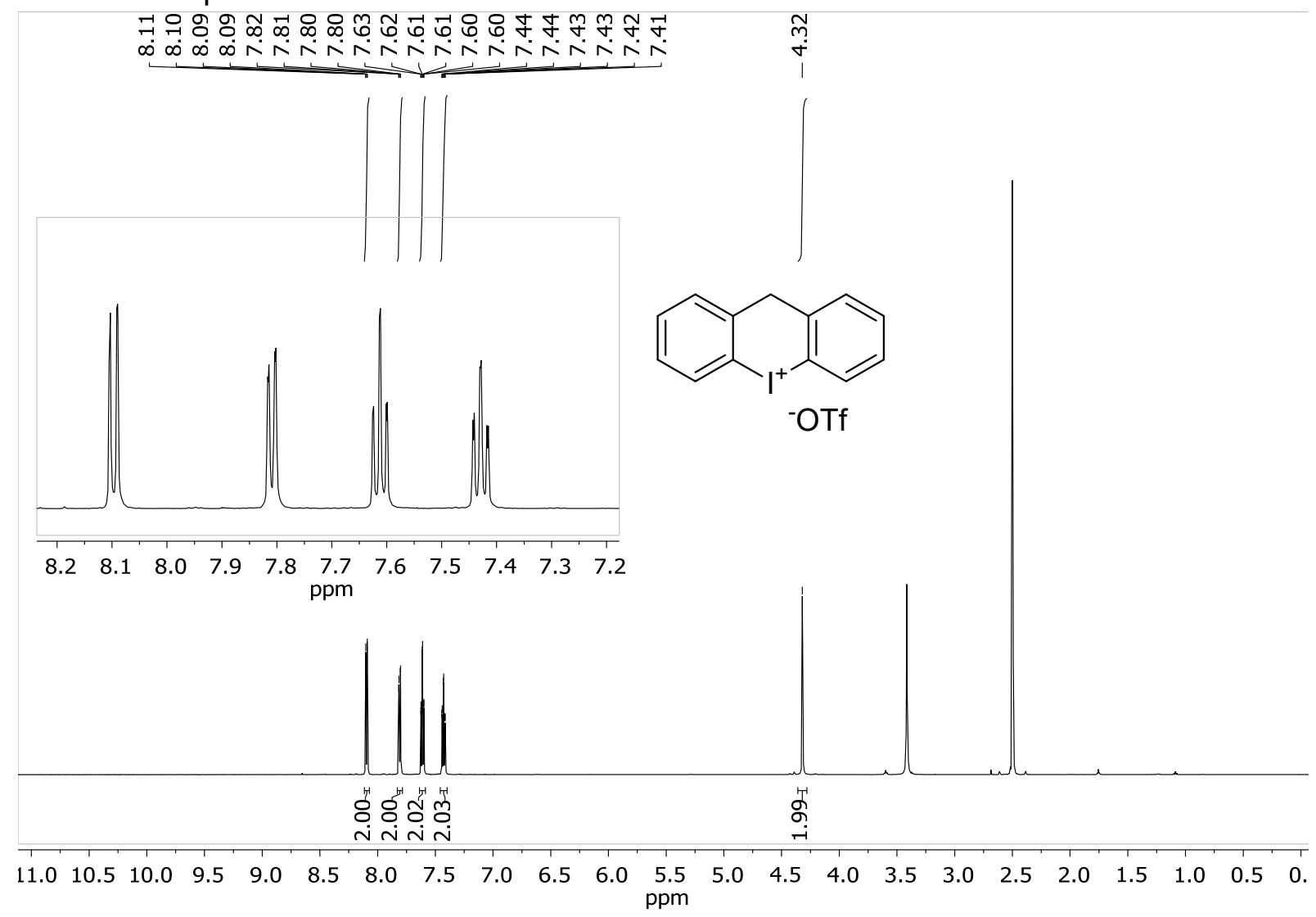

Figure S26: $600 \mathrm{MHz}{ }^{1} \mathrm{H}-\mathrm{NMR}$ spectrum of compound $7 \mathrm{a}$.

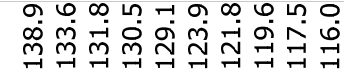
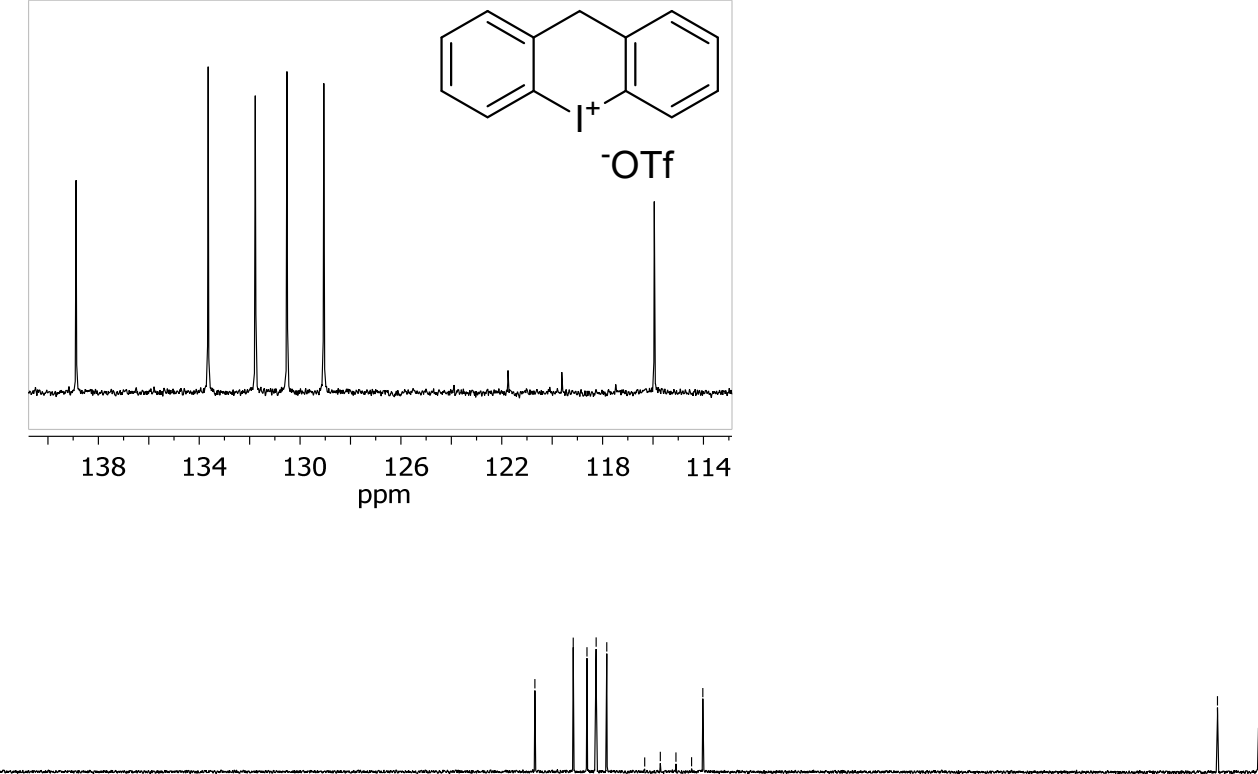

$\begin{array}{llllllllllllllllllllll}210 & 200 & 190 & 180 & 170 & 160 & 150 & 140 & 130 & 120 & 110 & 100 & 90 & 80 & 70 & 60 & 50 & 40 & 30 & 20 & 10 & 0\end{array}$

Figure S27: $151 \mathrm{MHz}{ }^{13} \mathrm{C}-N M R$ spectrum of compound $7 a$. 


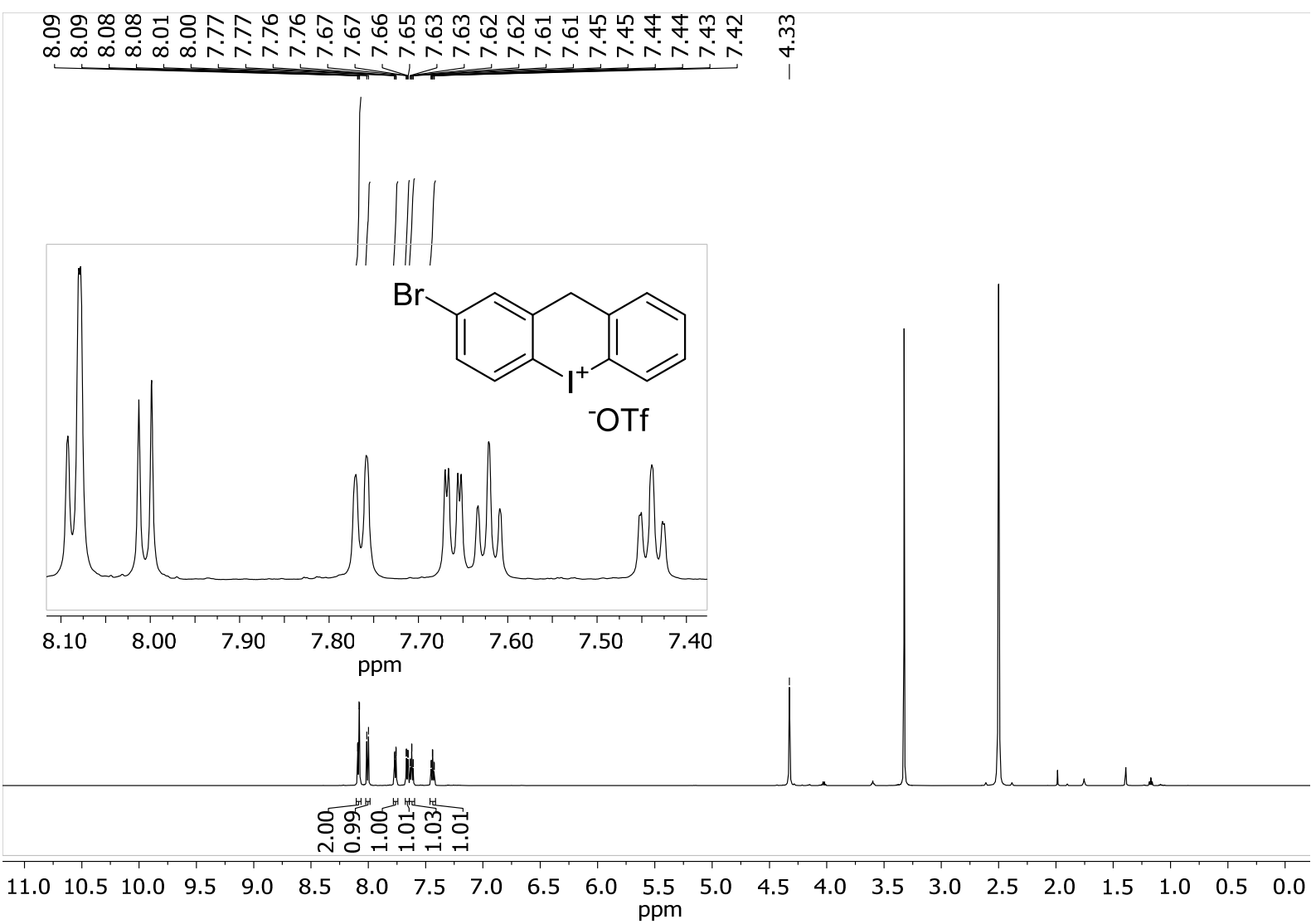

Figure S28: $600 \mathrm{MHz}{ }^{1} \mathrm{H}-\mathrm{NMR}$ spectrum of compound $\mathbf{7 b}$.
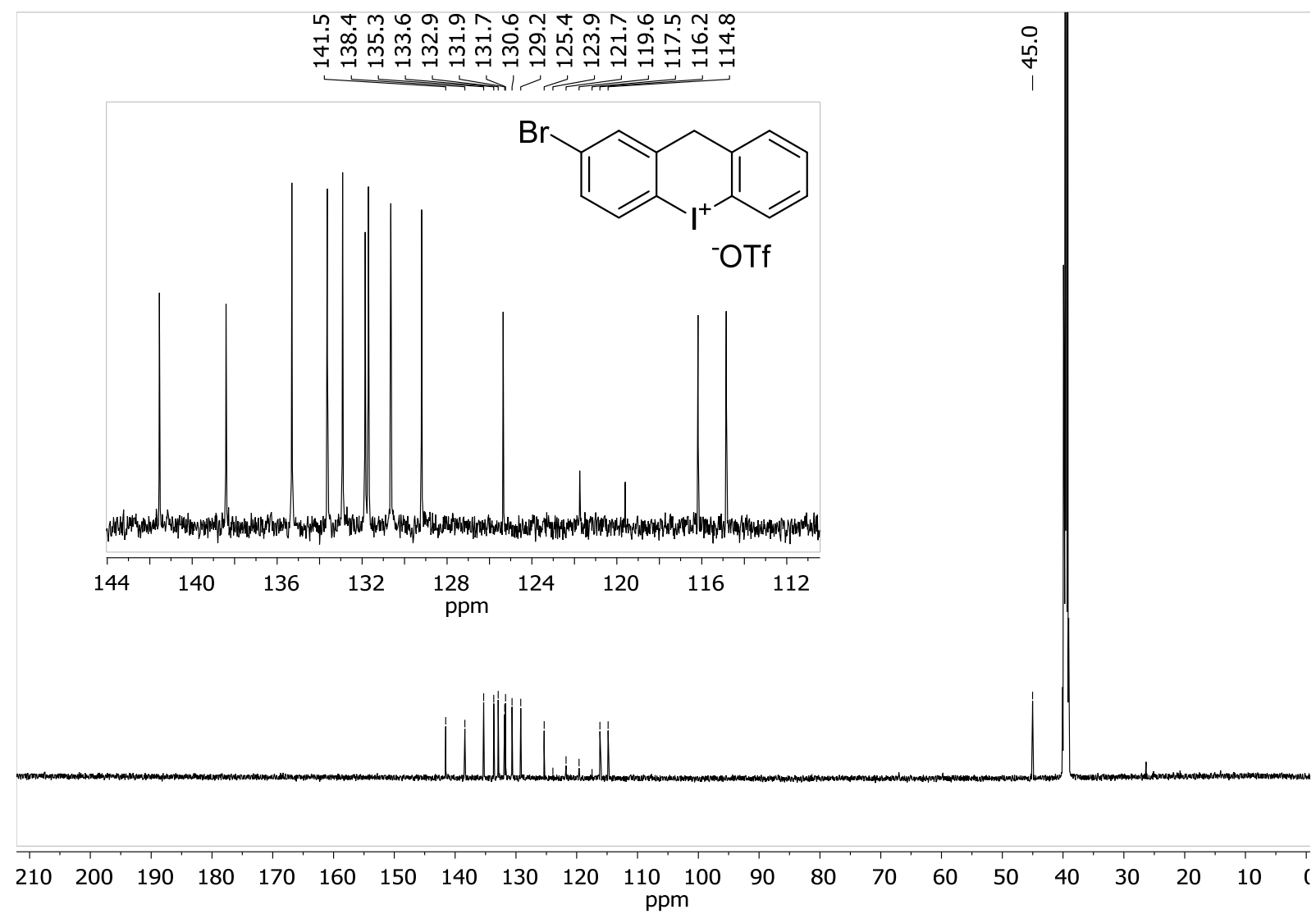

Figure S29: $151 \mathrm{MHz}{ }^{13} \mathrm{C}-\mathrm{NMR}$ spectrum of compound $7 \mathrm{~b}$. 


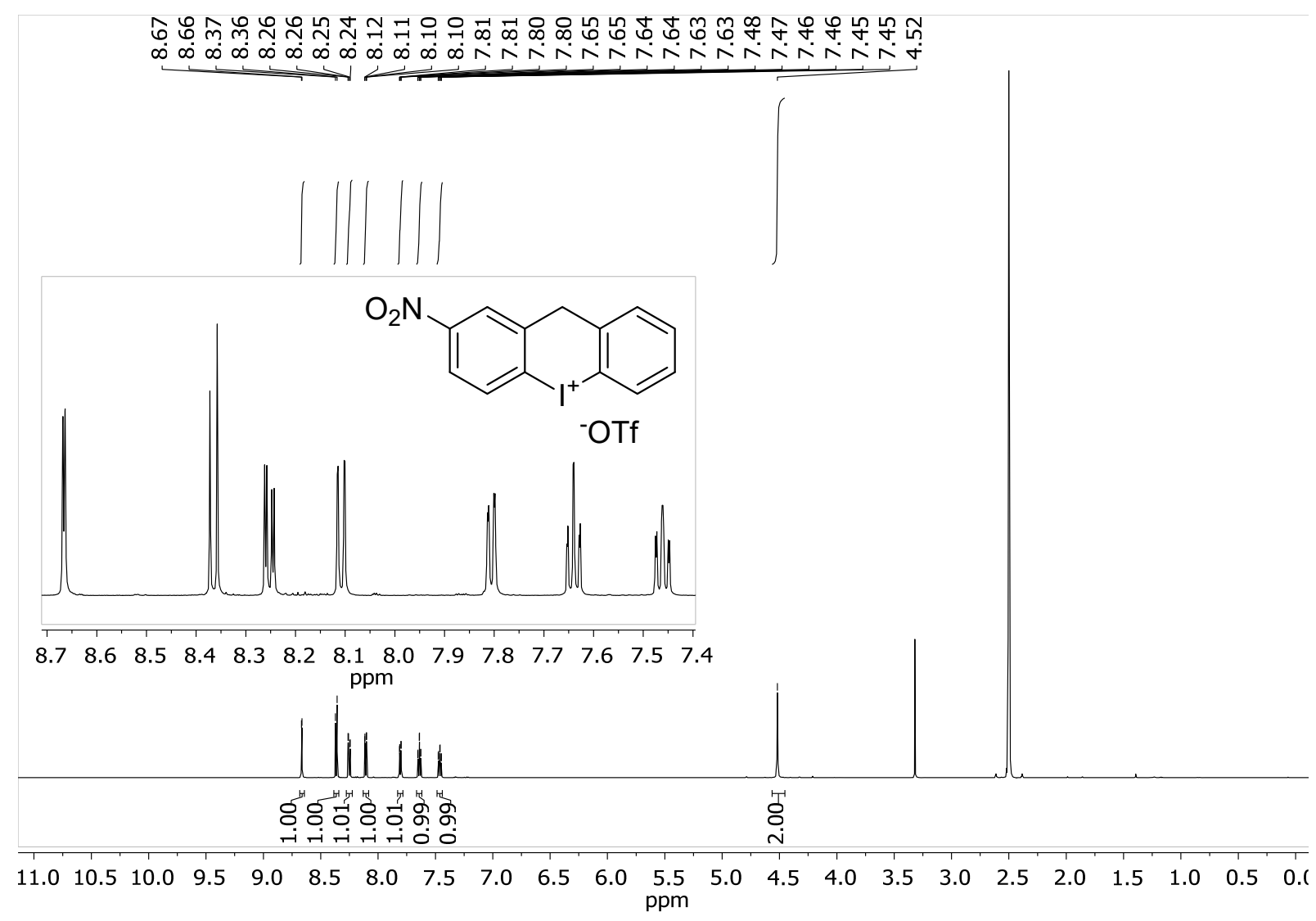

Figure S30: $600 \mathrm{MHz}{ }^{1} \mathrm{H}-\mathrm{NMR}$ spectrum of compound $7 \mathrm{c}$.

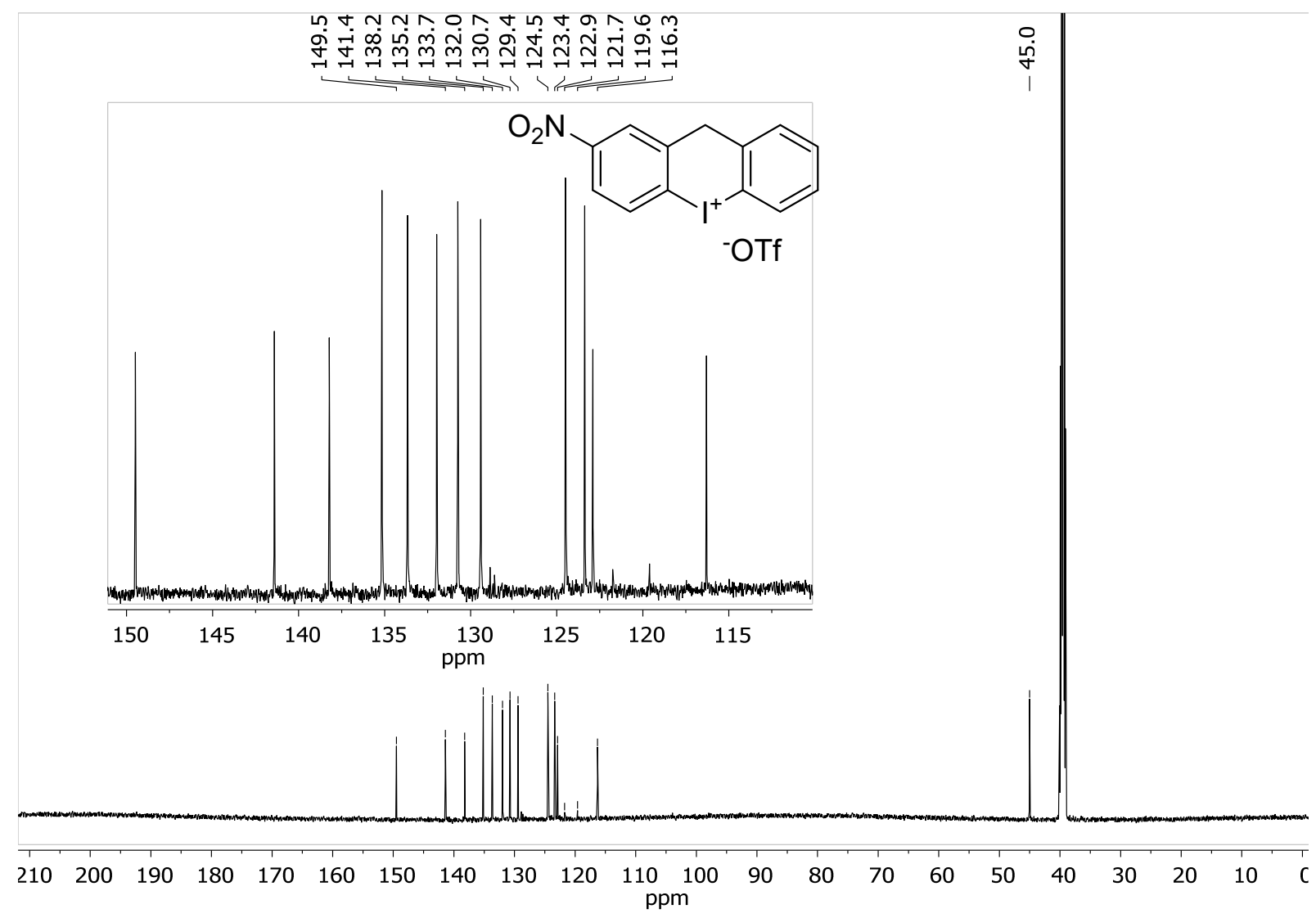

Figure S31: $151 \mathrm{MHz}{ }^{13} \mathrm{C}-\mathrm{NMR}$ spectrum of compound $7 \mathrm{c}$. 


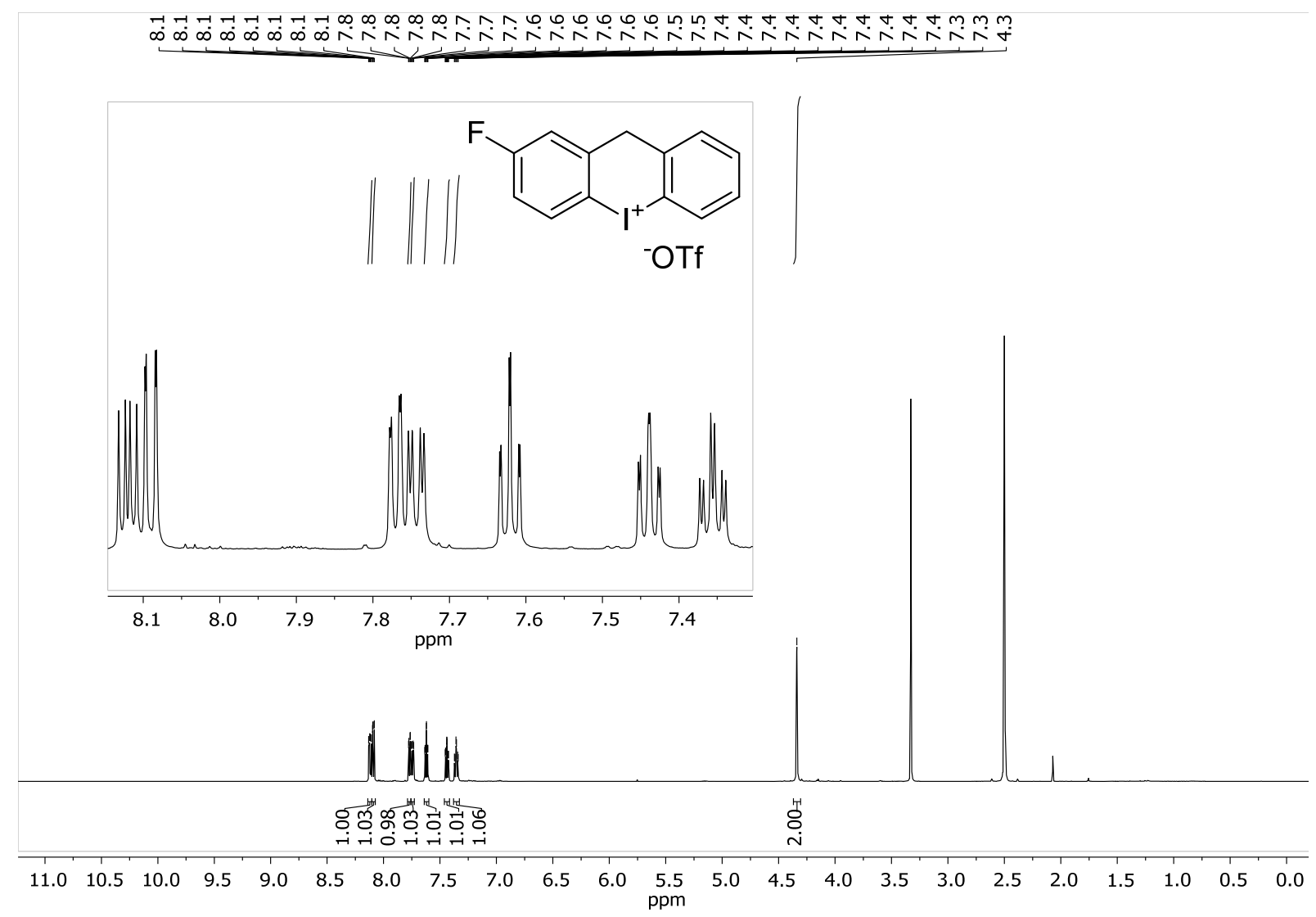

Figure S32: $600 \mathrm{MHz}{ }^{1} \mathrm{H}-\mathrm{NMR}$ spectrum of compound $7 d$.

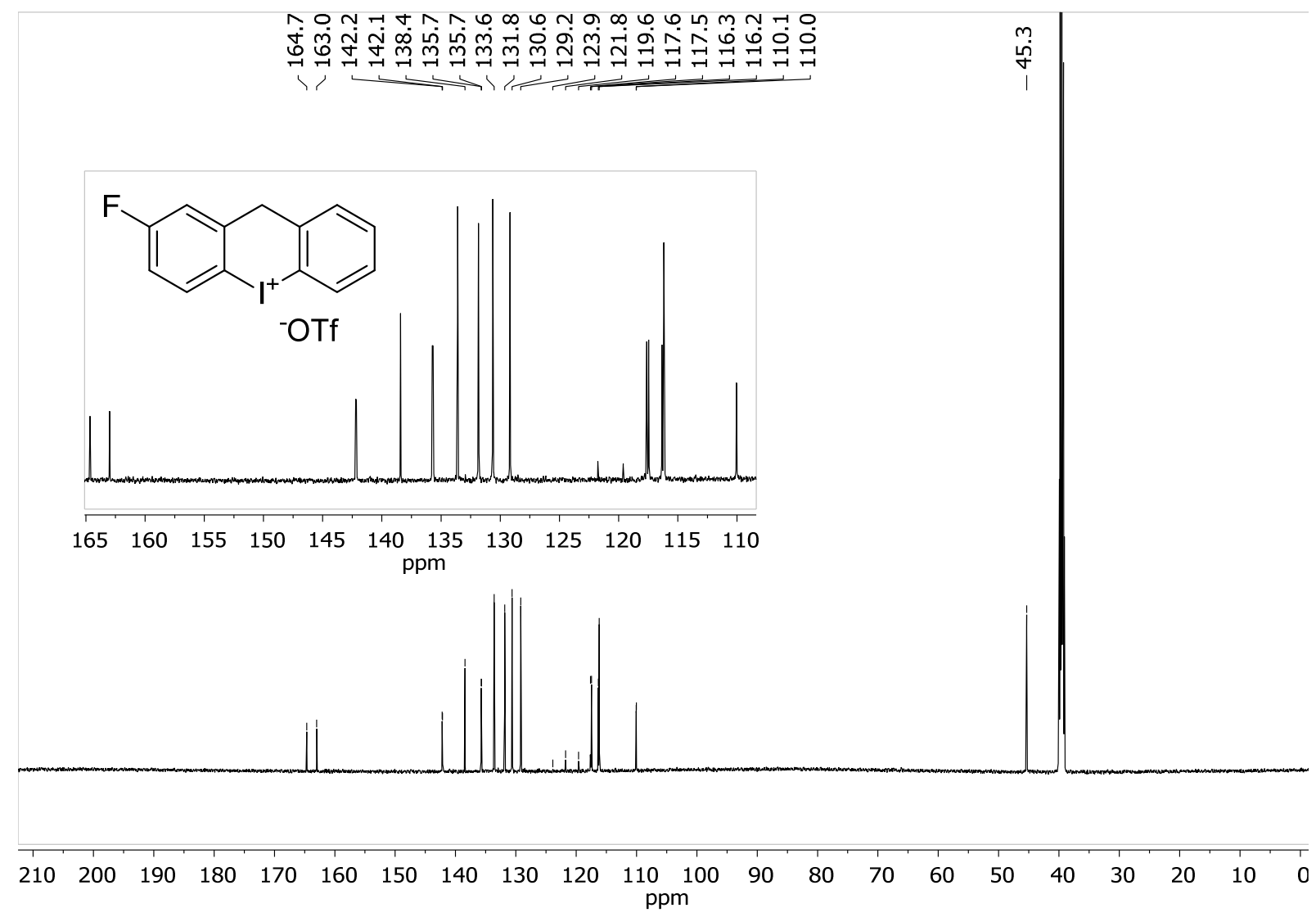

Figure S33: $151 \mathrm{MHz}{ }^{13} \mathrm{C}-\mathrm{NMR}$ spectrum of compound $7 d$. 


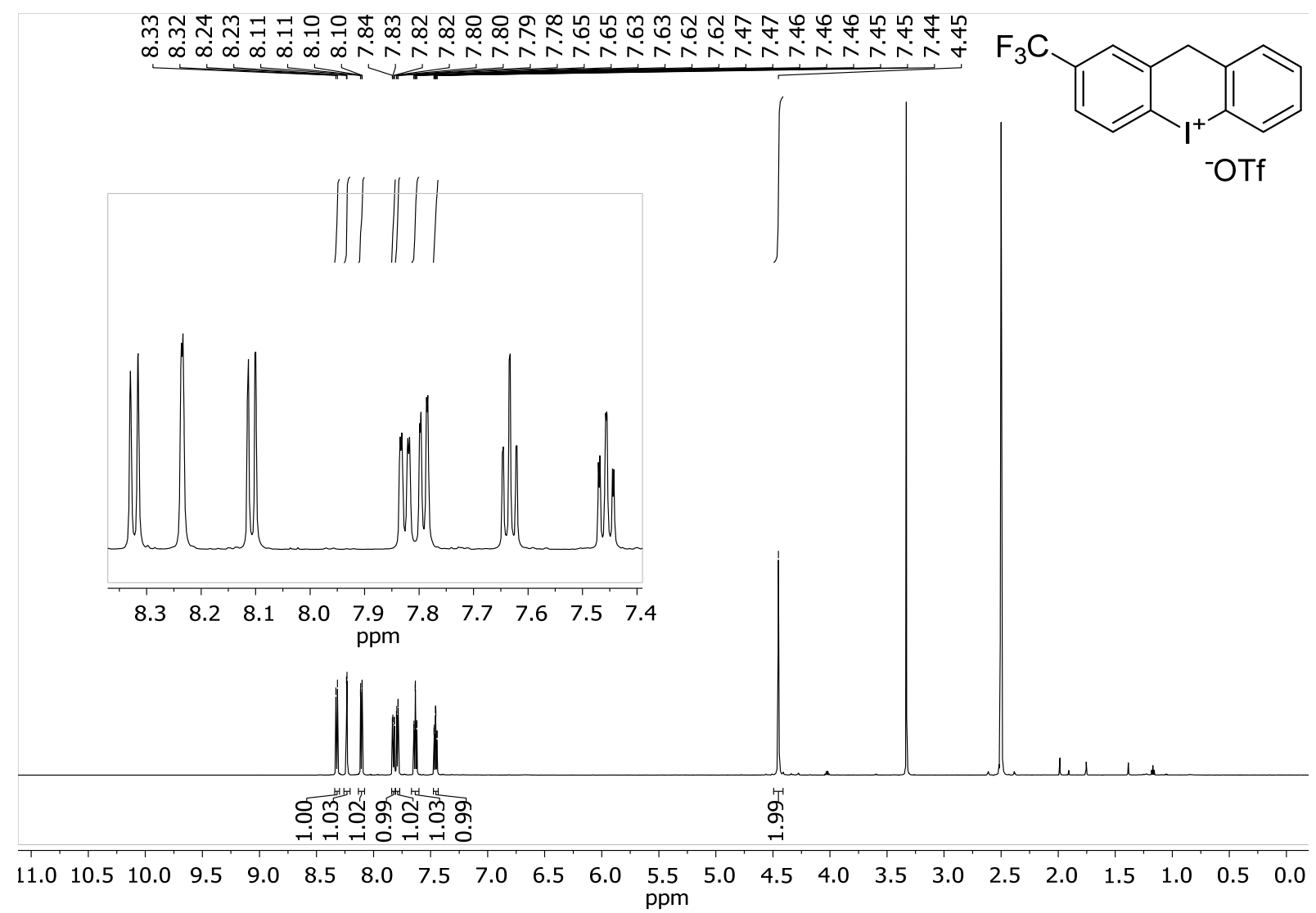

Figure S34: $600 \mathrm{MHz}{ }^{1} \mathrm{H}-\mathrm{NMR}$ spectrum of compound 7 .

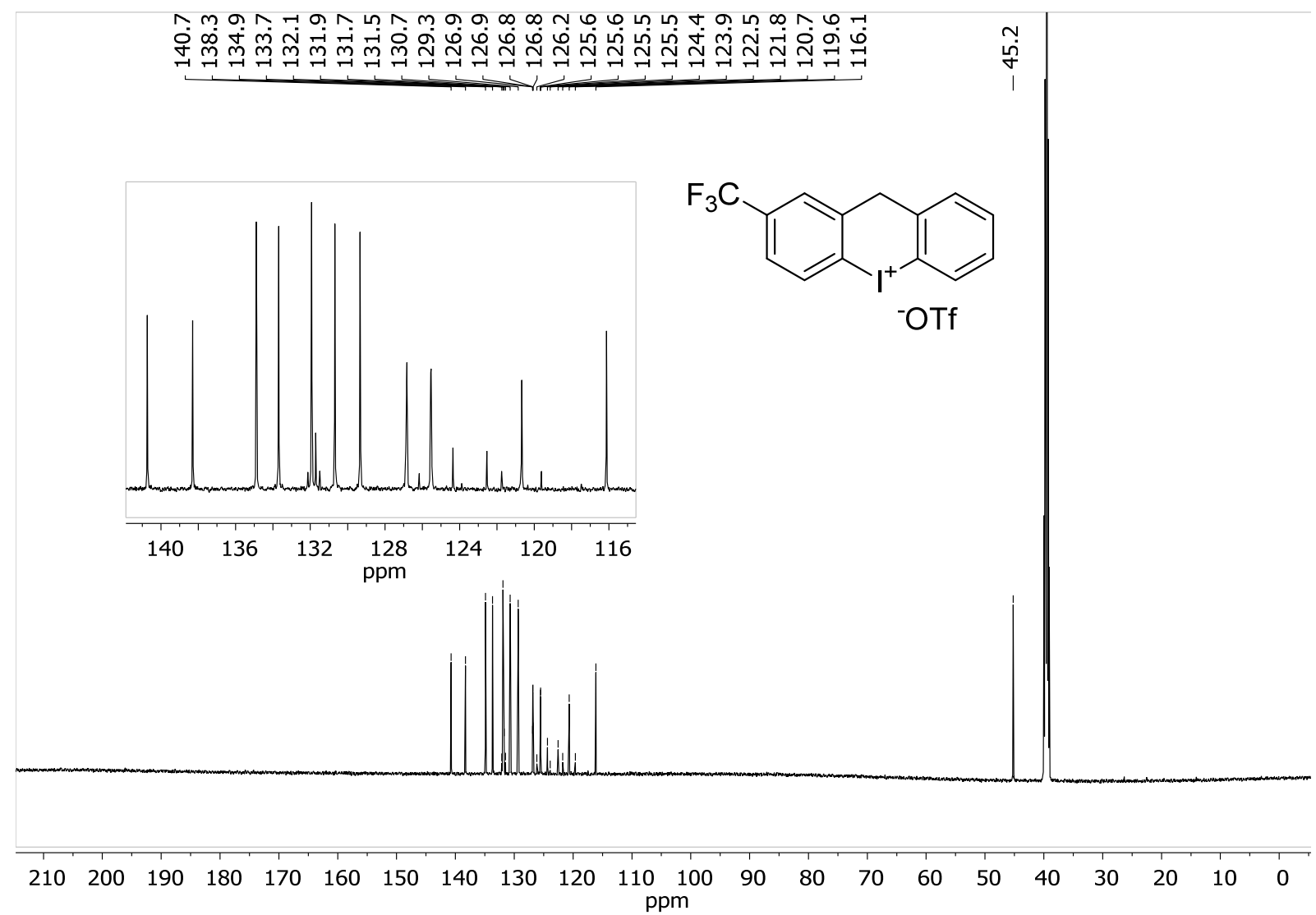

Figure S35: $151 \mathrm{MHz}{ }^{13} \mathrm{C}-\mathrm{NMR}$ spectrum of compound $7 e$. 


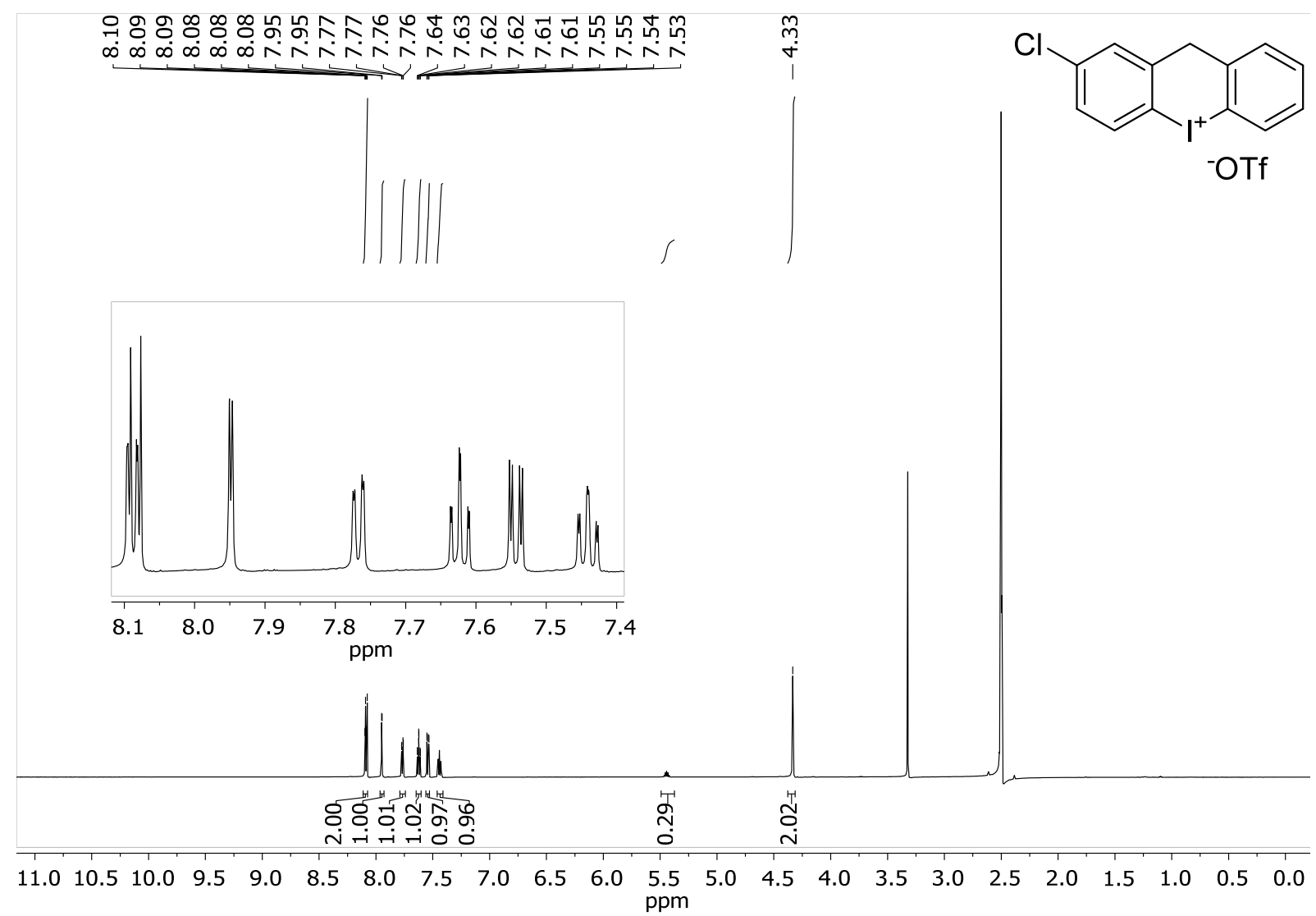

Figure S36: $600 \mathrm{MHz}{ }^{1} \mathrm{H}-\mathrm{NMR}$ spectrum of compound $7 f$.

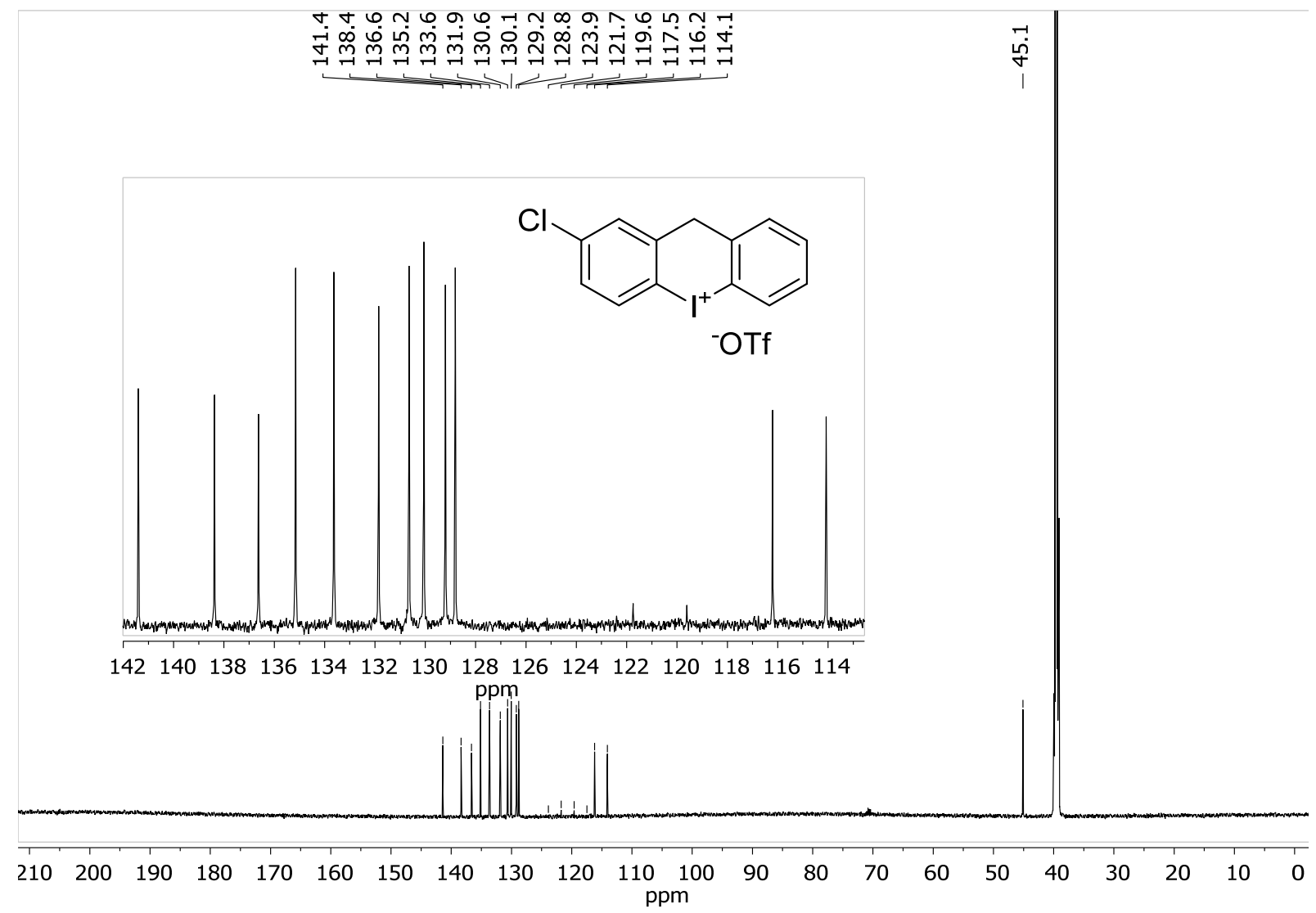

Figure S37: $151 \mathrm{MHz}{ }^{13} \mathrm{C}-\mathrm{NMR}$ spectrum of compound $7 f$. 


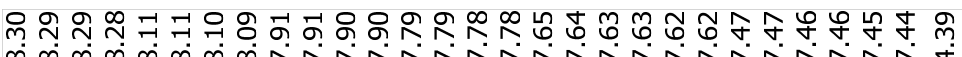

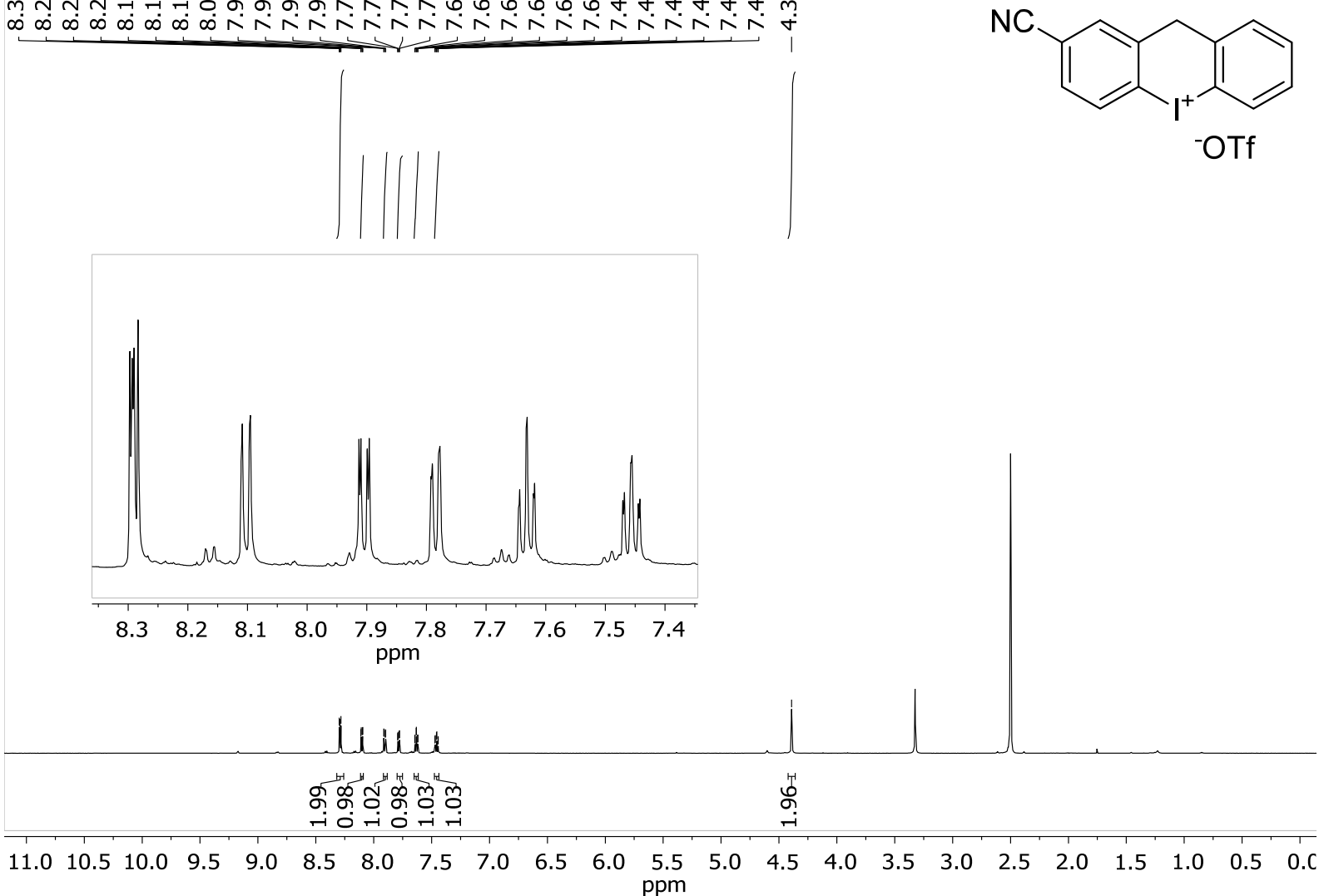

Figure S38: $600 \mathrm{MHz}{ }^{1} \mathrm{H}-\mathrm{NMR}$ spectrum of compound $\mathbf{7 g}$.

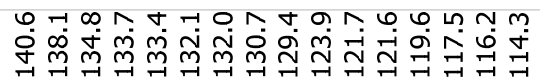
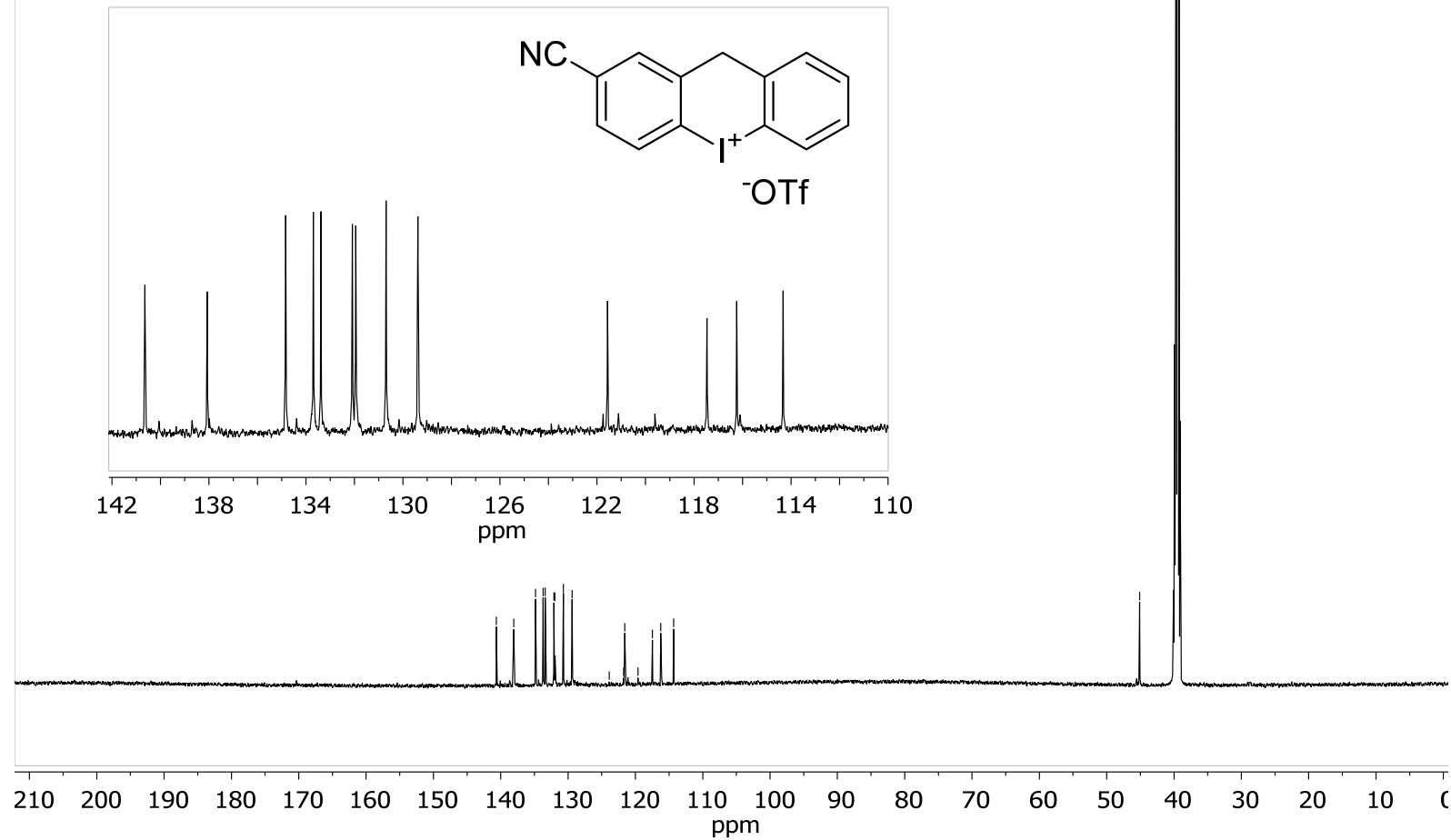

Figure S39: $151 \mathrm{MHz}{ }^{13} \mathrm{C}-\mathrm{NMR}$ spectrum of compound $\mathbf{7 g}$. 


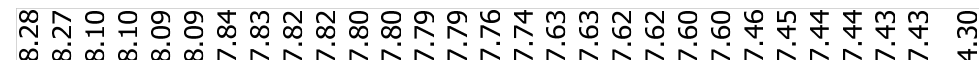

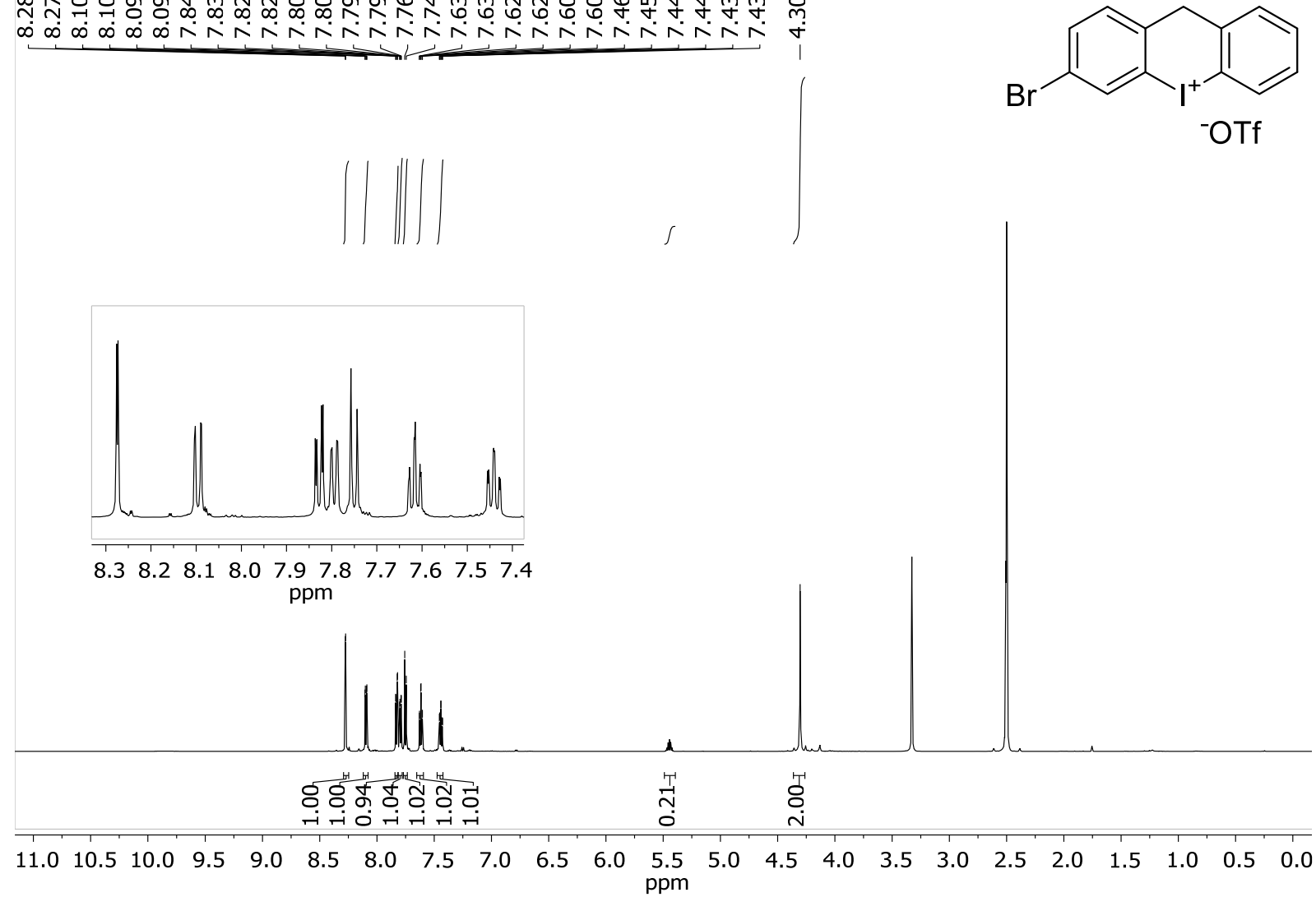

Figure S40: $600 \mathrm{MHz}^{1} \mathrm{H}-\mathrm{NMR}$ spectrum of compound $7 \mathrm{~h}$.

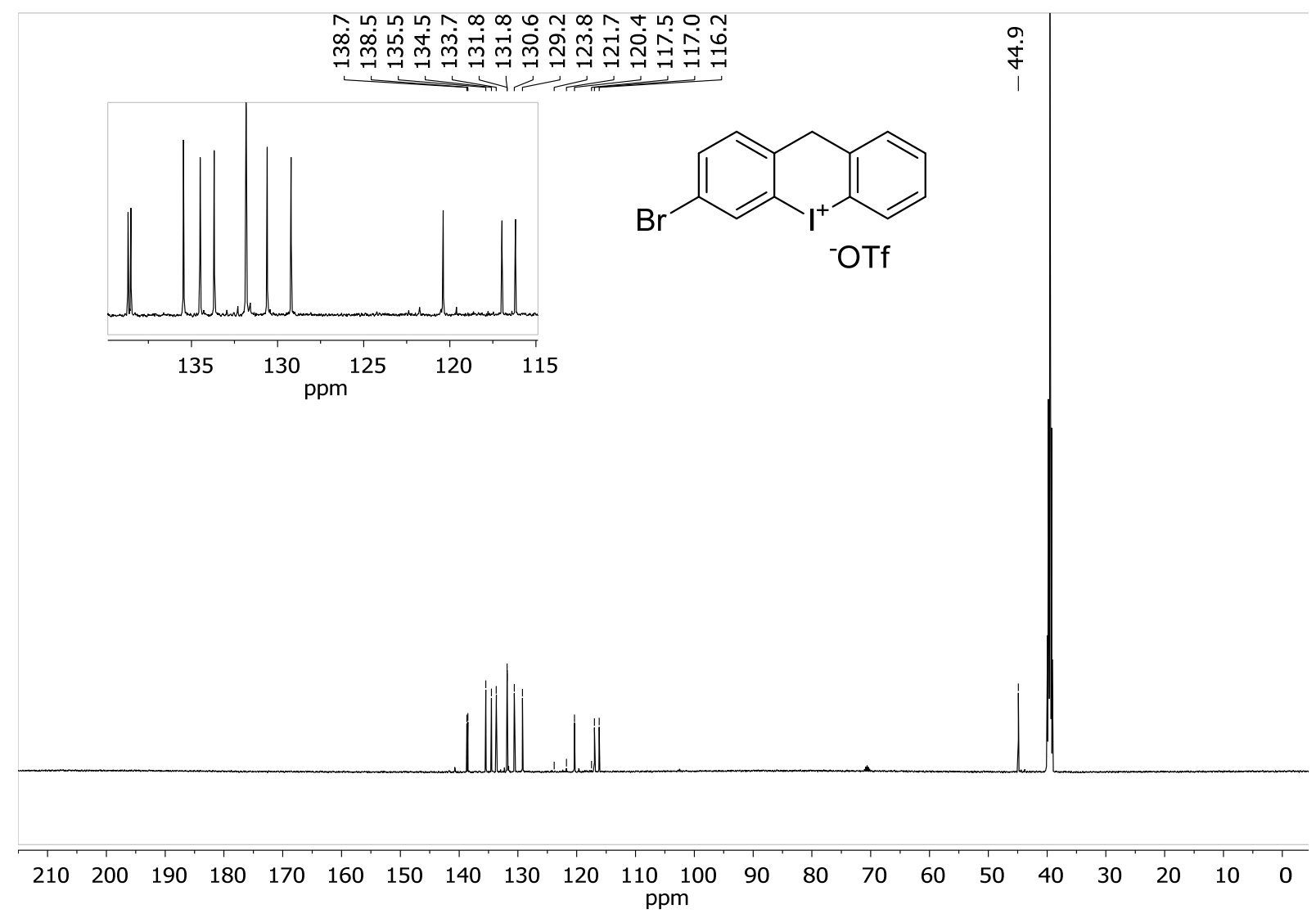

Figure S41: $151 \mathrm{MHz}{ }^{13} \mathrm{C}-\mathrm{NMR}$ spectrum of compound $7 \mathrm{~h}$. 


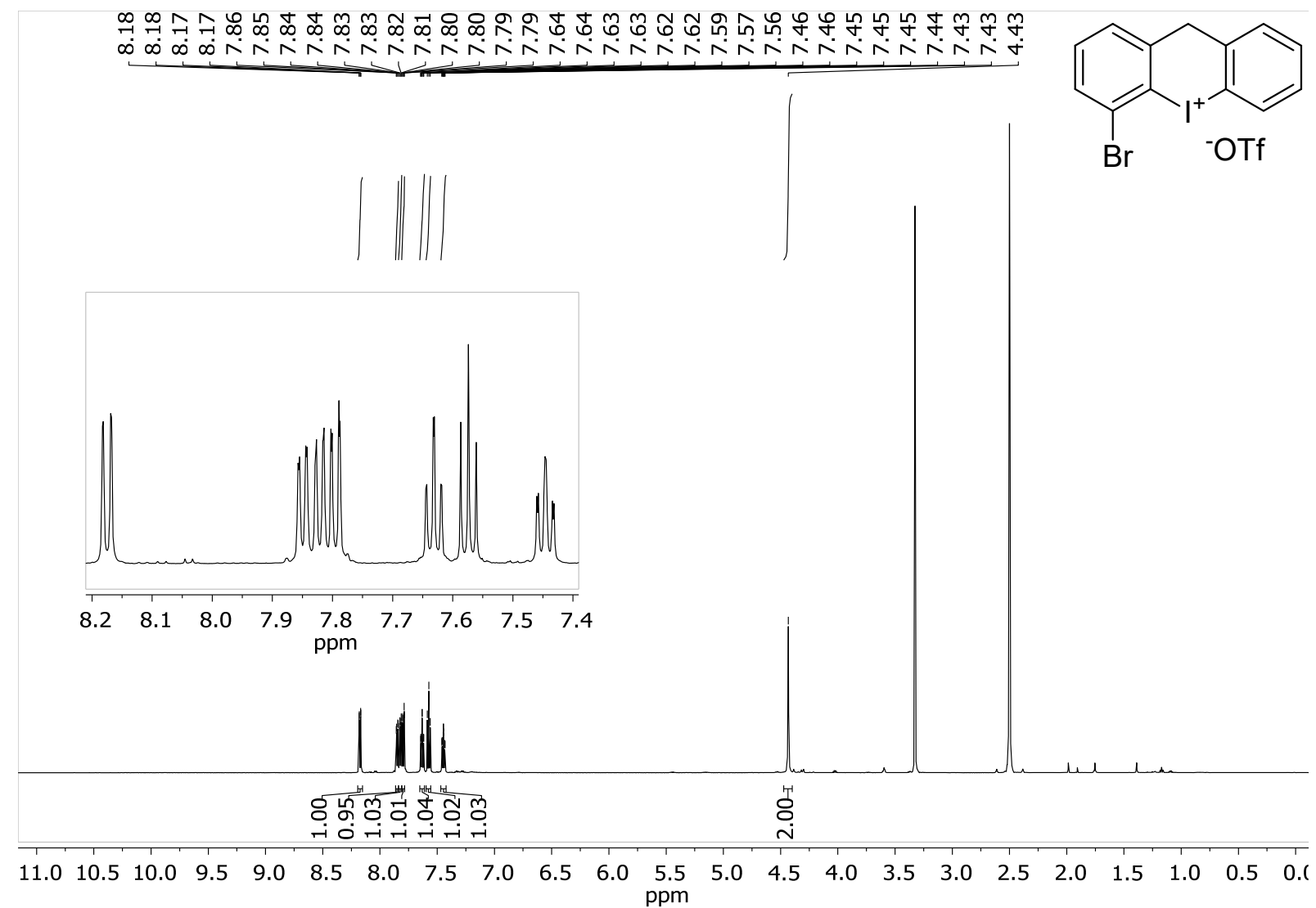

Figure S42: $600 \mathrm{MHz}{ }^{1} \mathrm{H}-\mathrm{NMR}$ spectrum of compound 7 i.

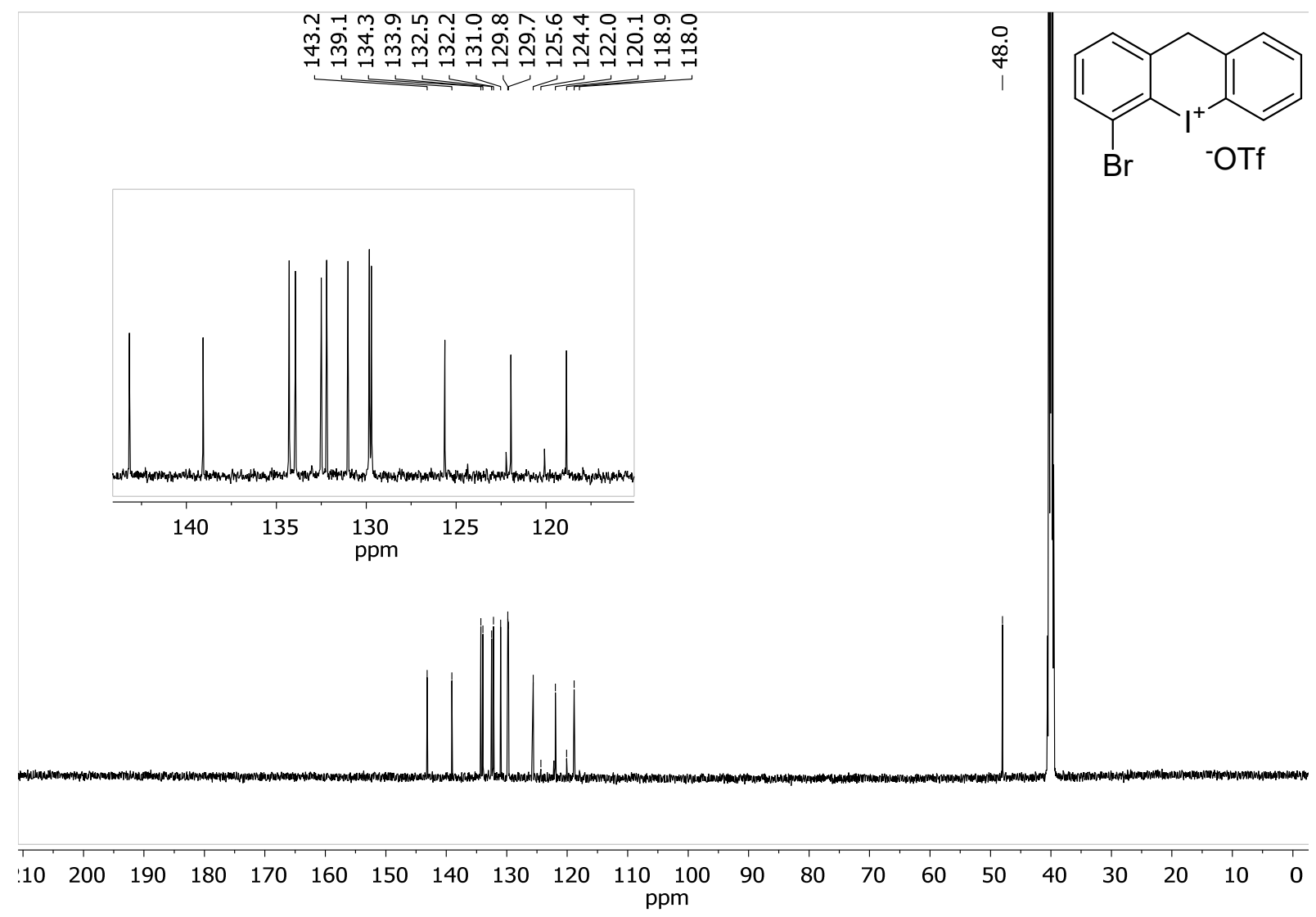

Figure S43: $151 \mathrm{MHz}{ }^{13} \mathrm{C}-\mathrm{NMR}$ spectrum of compound 7i. 


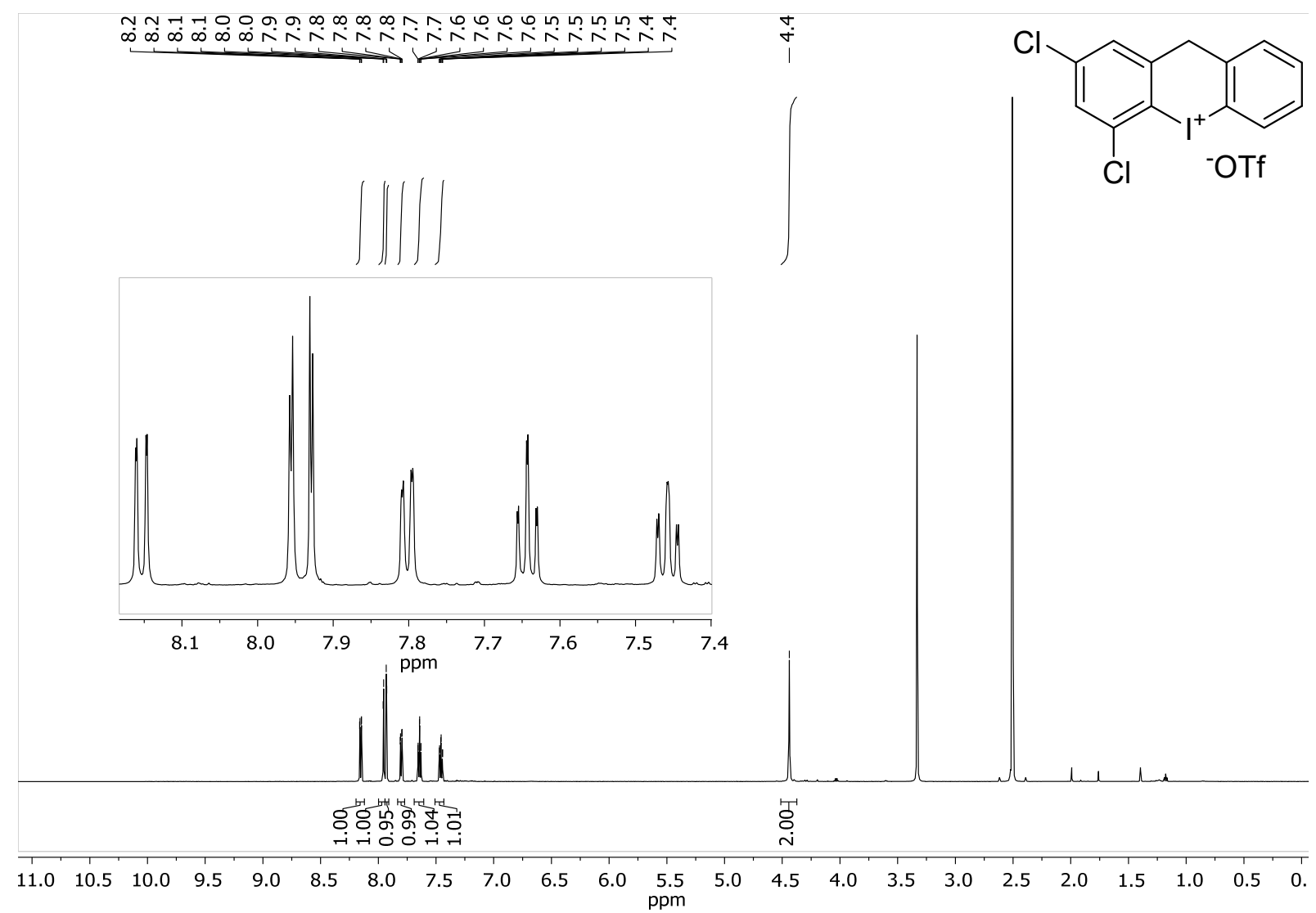

Figure S44: $600 \mathrm{MHz}{ }^{1} \mathrm{H}-\mathrm{NMR}$ spectrum of compound 7 j.
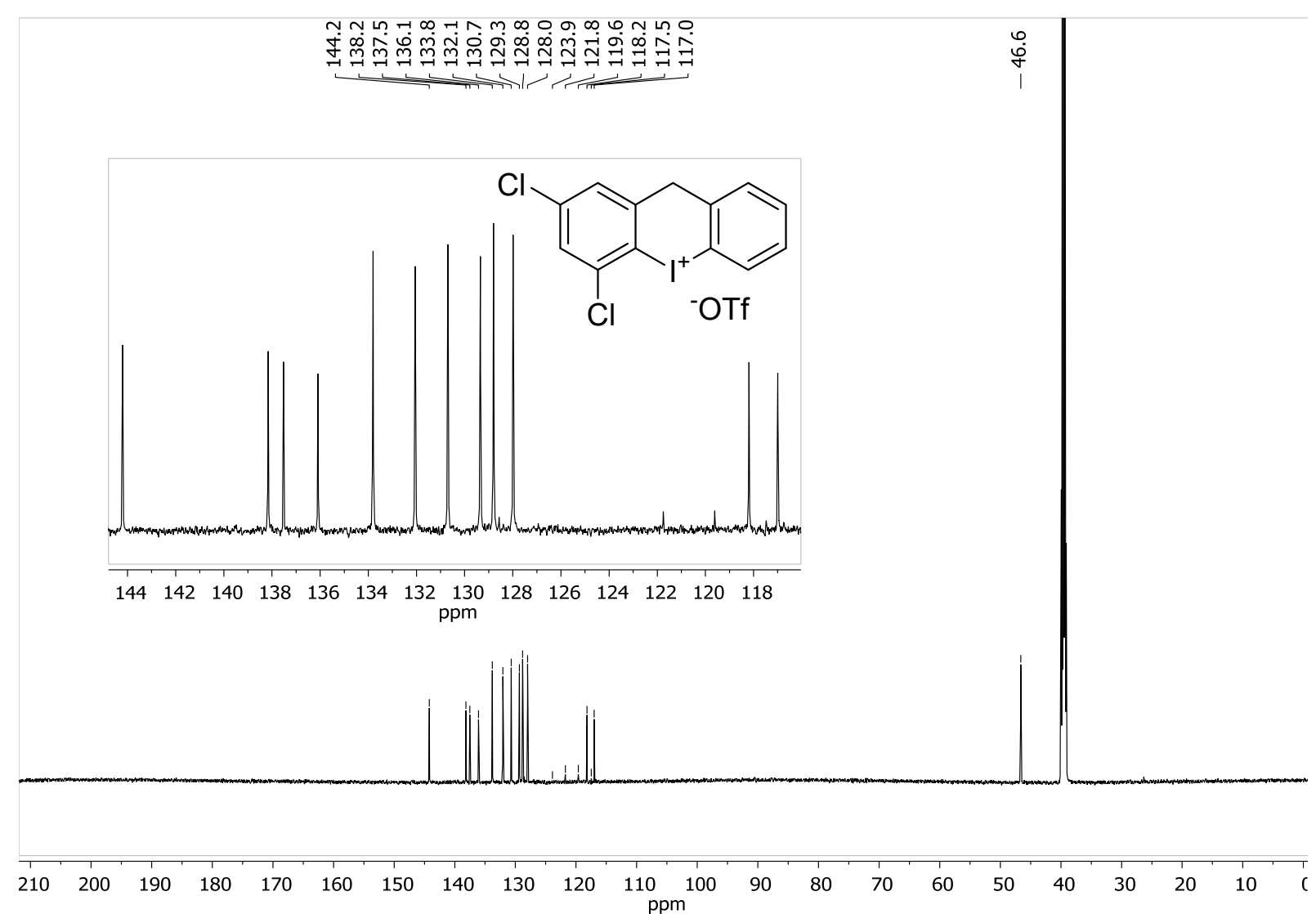

Figure S45: $151 \mathrm{MHz}{ }^{13} \mathrm{C}-N M R$ spectrum of compound 7 j. 


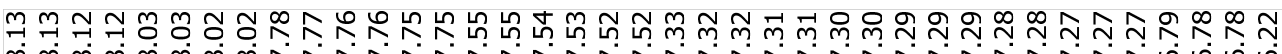

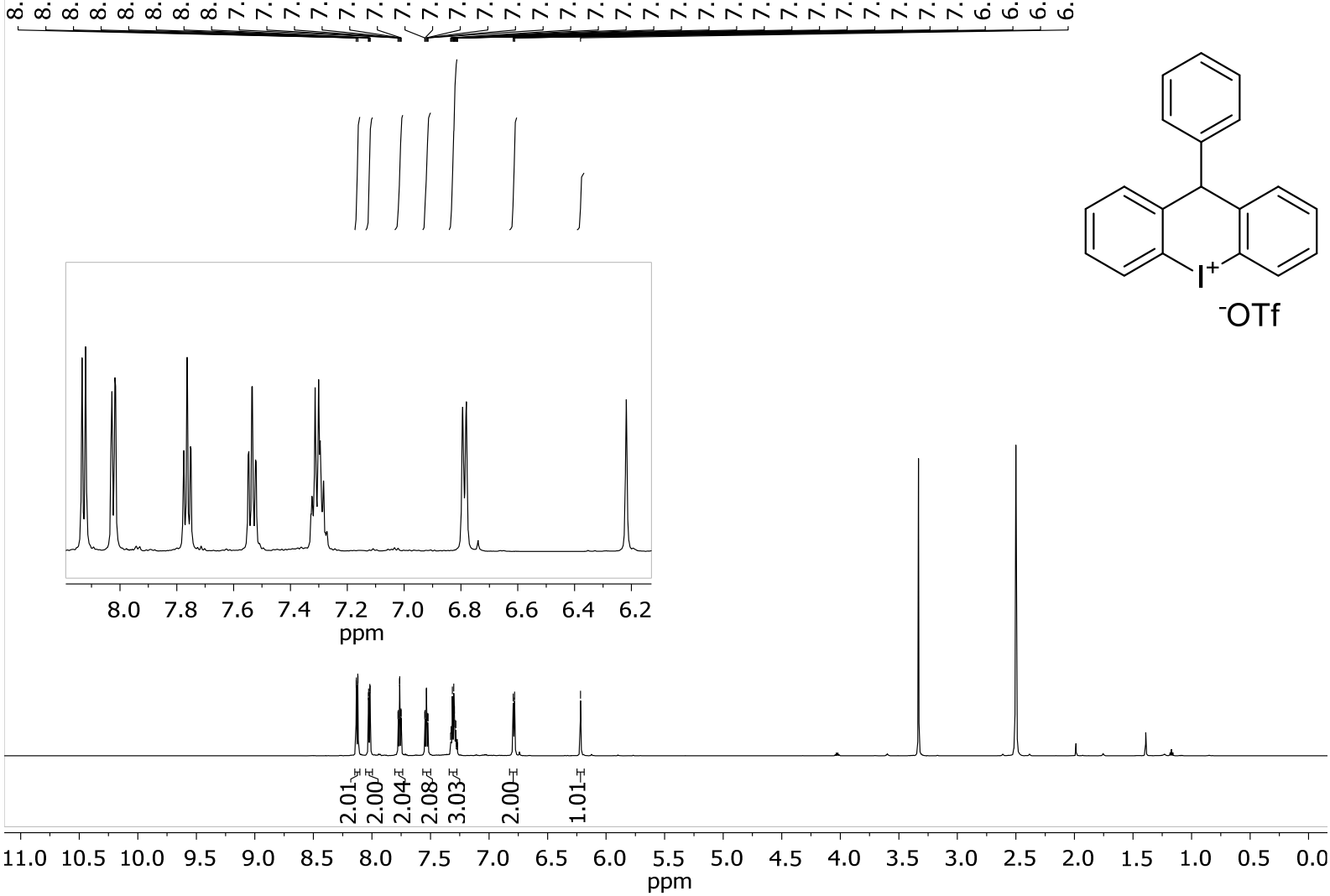

Figure S46: $600 \mathrm{MHz}{ }^{1} \mathrm{H}-\mathrm{NMR}$ spectrum of compound $\mathbf{7 k}$.

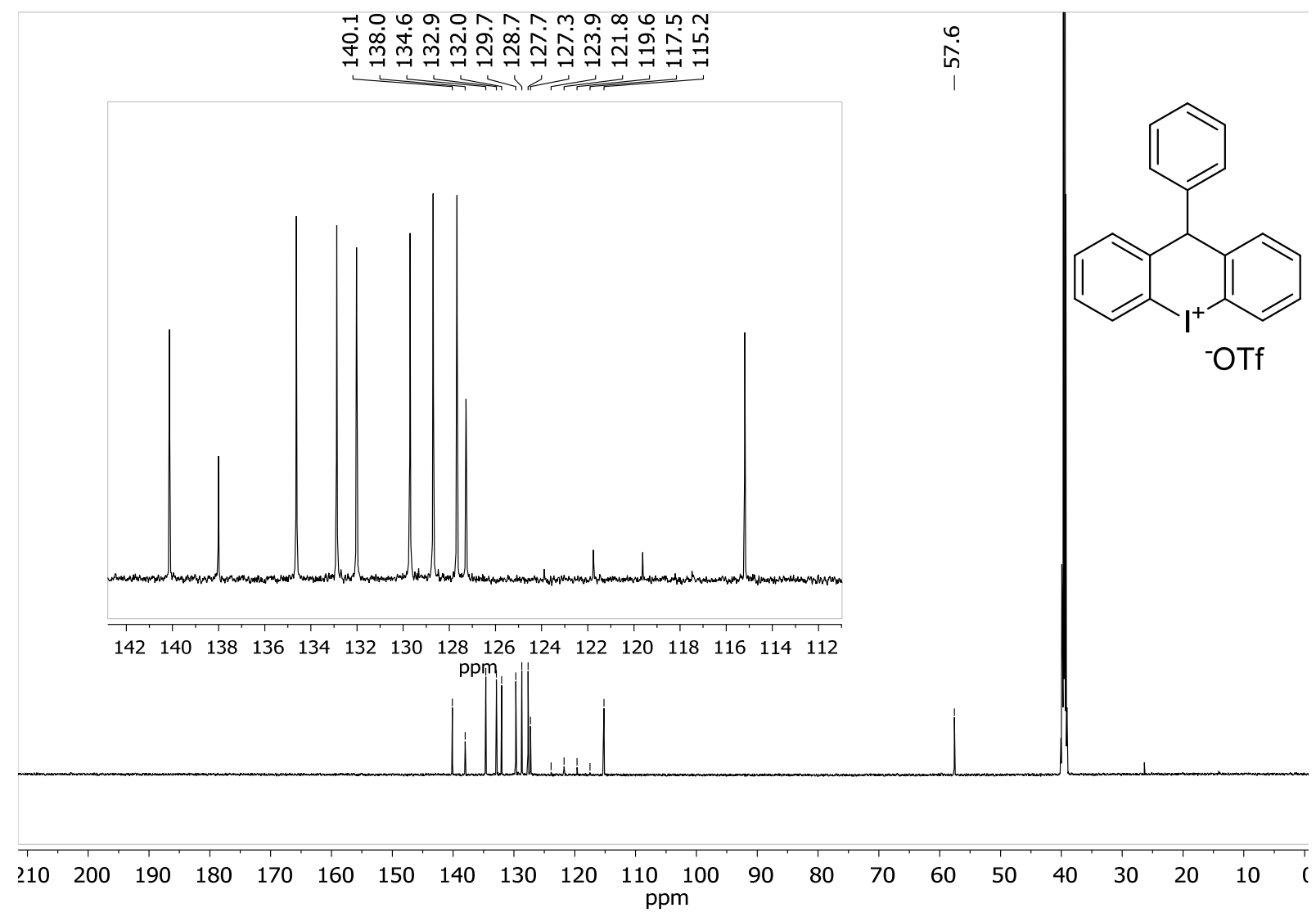

Figure S47: $151 \mathrm{MHz}{ }^{13} \mathrm{C}-N M R$ spectrum of compound $\mathbf{7 k}$. 


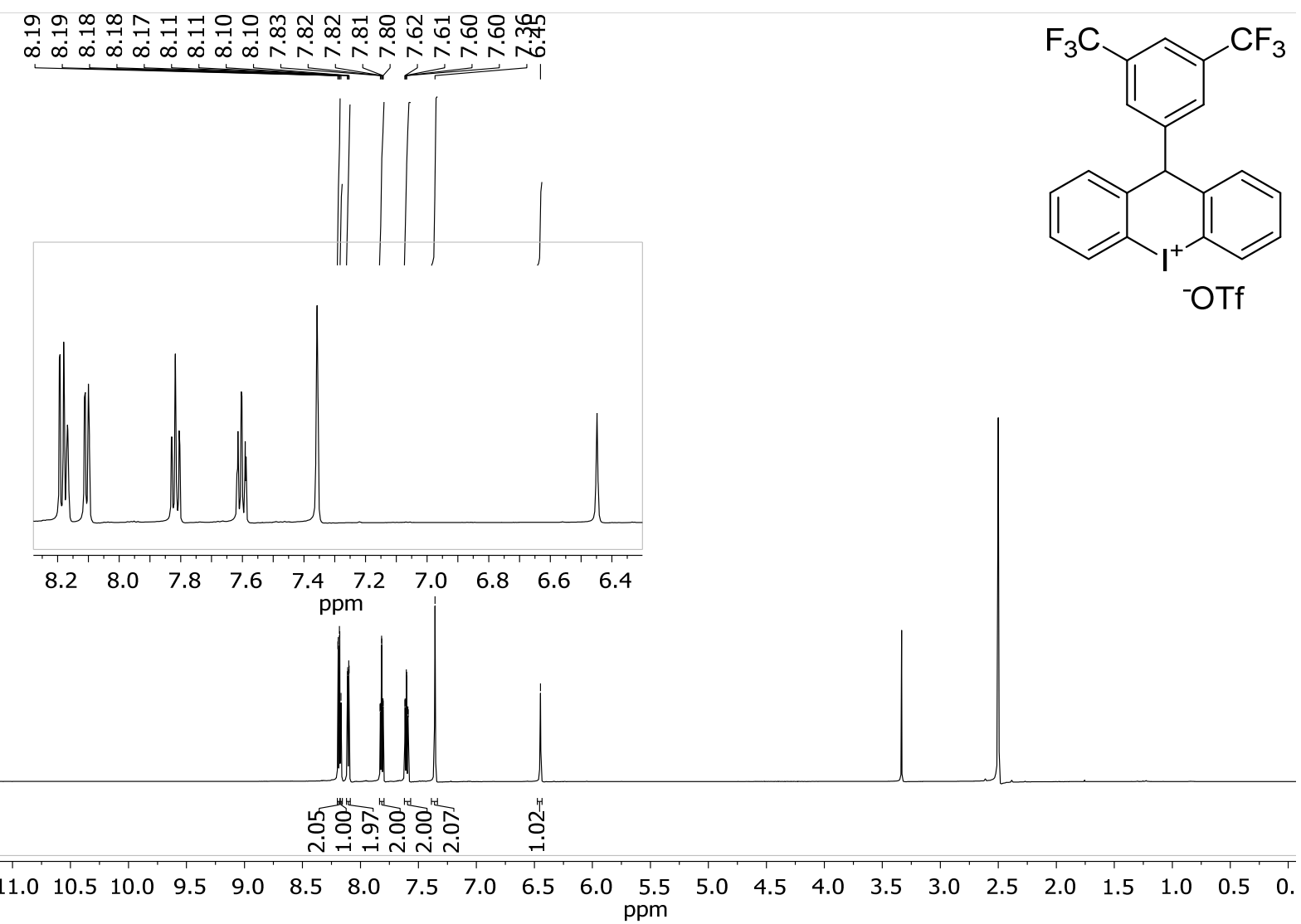

Figure S48: $600 \mathrm{MHz}{ }^{1} \mathrm{H}-\mathrm{NMR}$ spectrum of compound 7 .

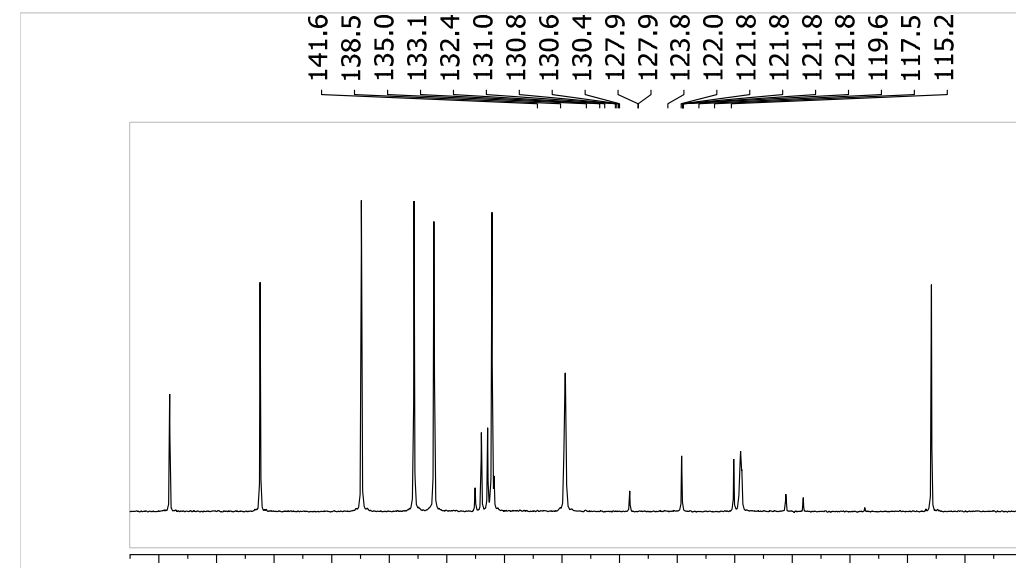

?̊.<smiles></smiles>

$\begin{array}{lllllllllllllll}142 & 140 & 138 & 136 & 134 & 132 & 130 & 128 & 126 & 124 & 122 & 120 & 118 & 116 & 114\end{array}$
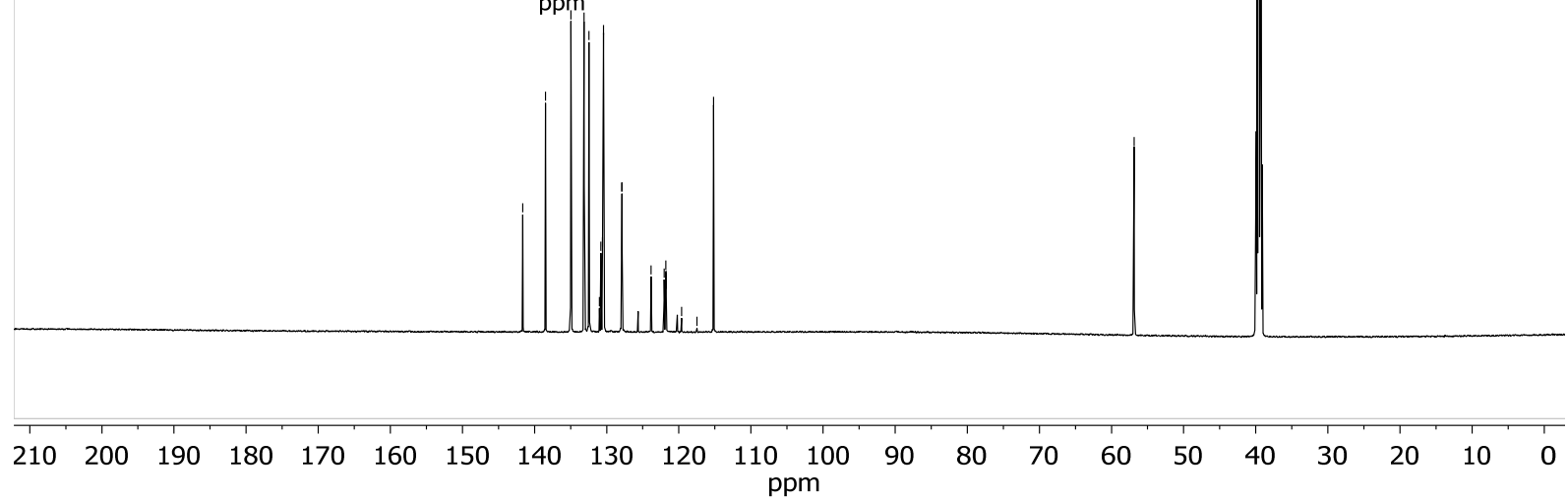

Figure S49: $151 \mathrm{MHz}{ }^{13} \mathrm{C}-\mathrm{NMR}$ spectrum of compound $7 \mathrm{I}$. 

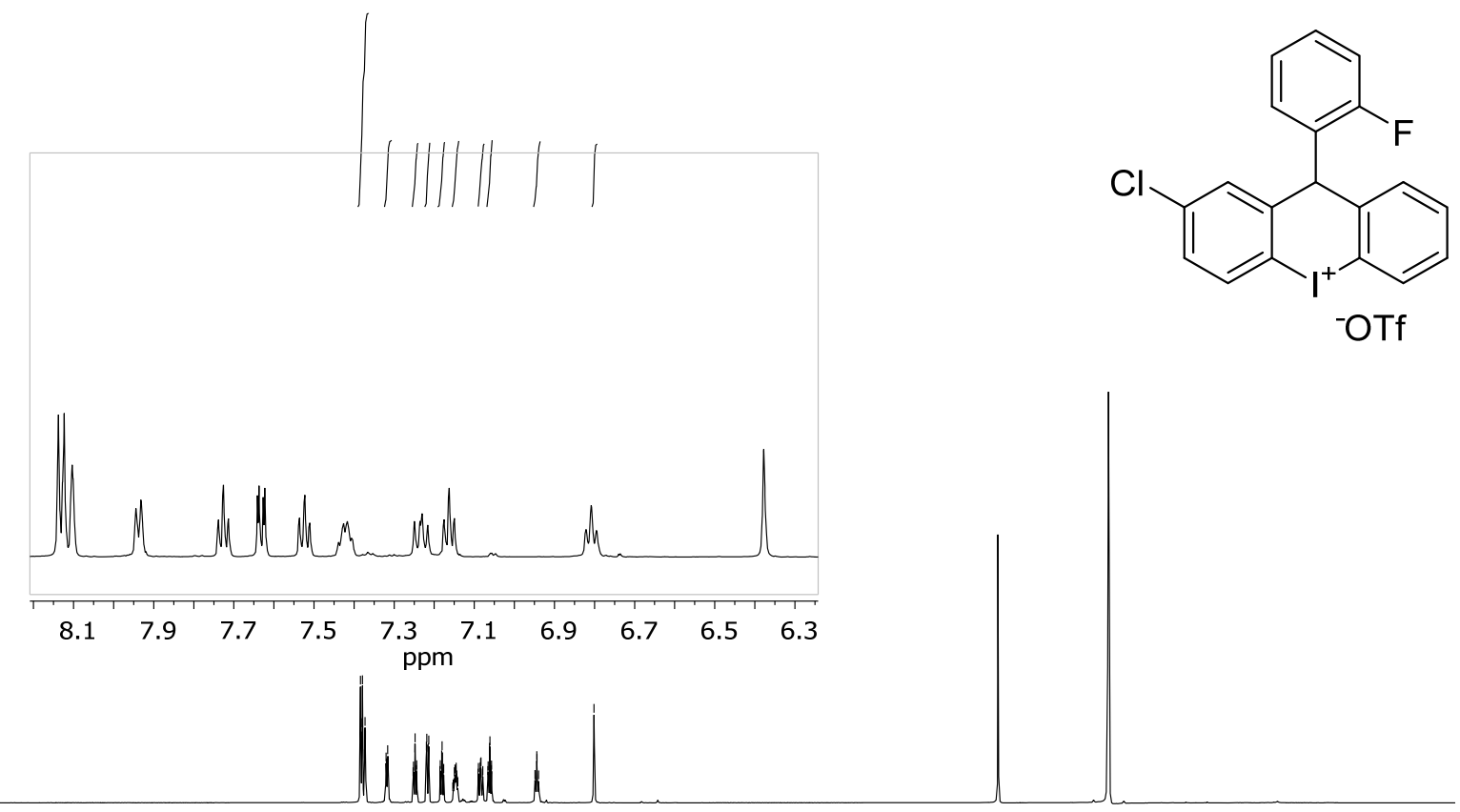

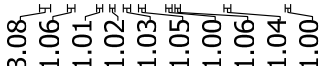

$\begin{array}{lllllllllllllllllllllll}11.0 & 10.5 & 10.0 & 9.5 & 9.0 & 8.5 & 8.0 & 7.5 & 7.0 & 6.5 & 6.0 & \begin{array}{r}5.5 \\ \mathrm{ppm}\end{array} & 5.0 & 4.5 & 4.0 & 3.5 & 3.0 & 2.5 & 2.0 & 1.5 & 1.0 & 0.5 & 0.1\end{array}$

Figure S50: $600 \mathrm{MHz}{ }^{1} \mathrm{H}-\mathrm{NMR}$ spectrum of compound $7 \mathrm{~m}$.

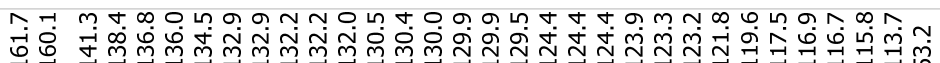

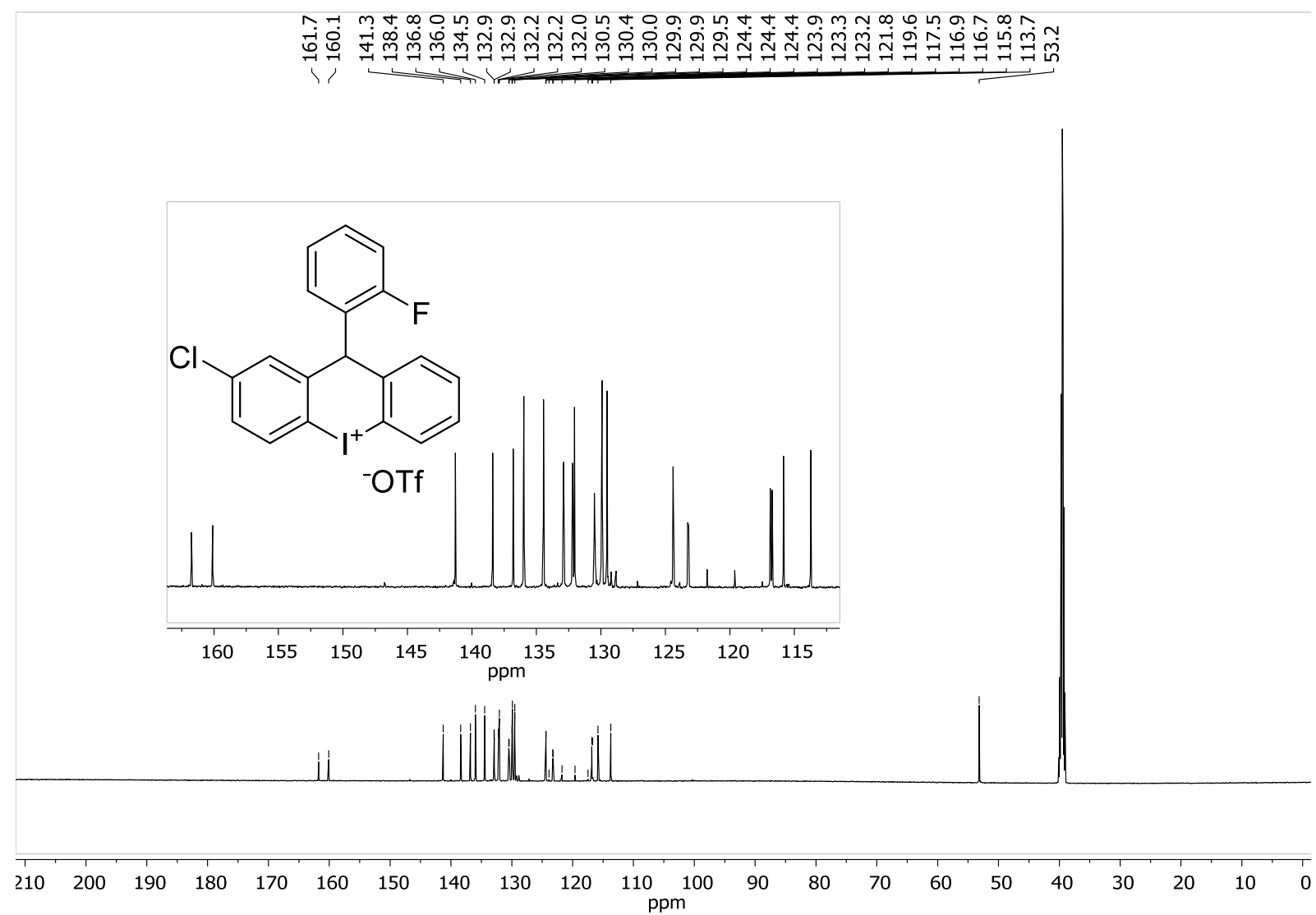

Figure S51: $151 \mathrm{MHz}{ }^{13} \mathrm{C}-\mathrm{NMR}$ spectrum of compound $7 \mathrm{~m}$. 


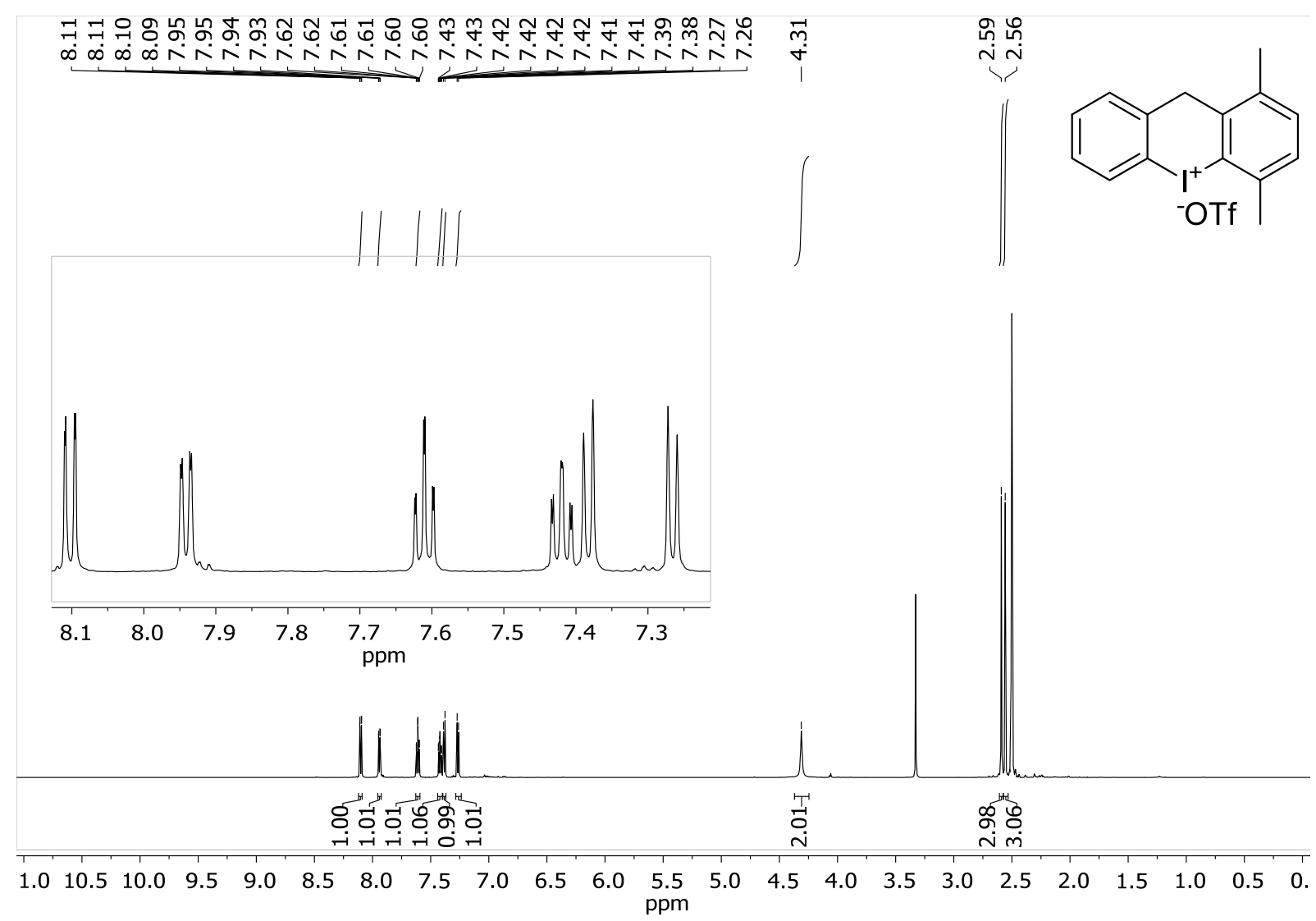

Figure S52: $600 \mathrm{MHz}{ }^{1} \mathrm{H}-\mathrm{NMR}$ spectrum of compound $\mathbf{7 n}$.

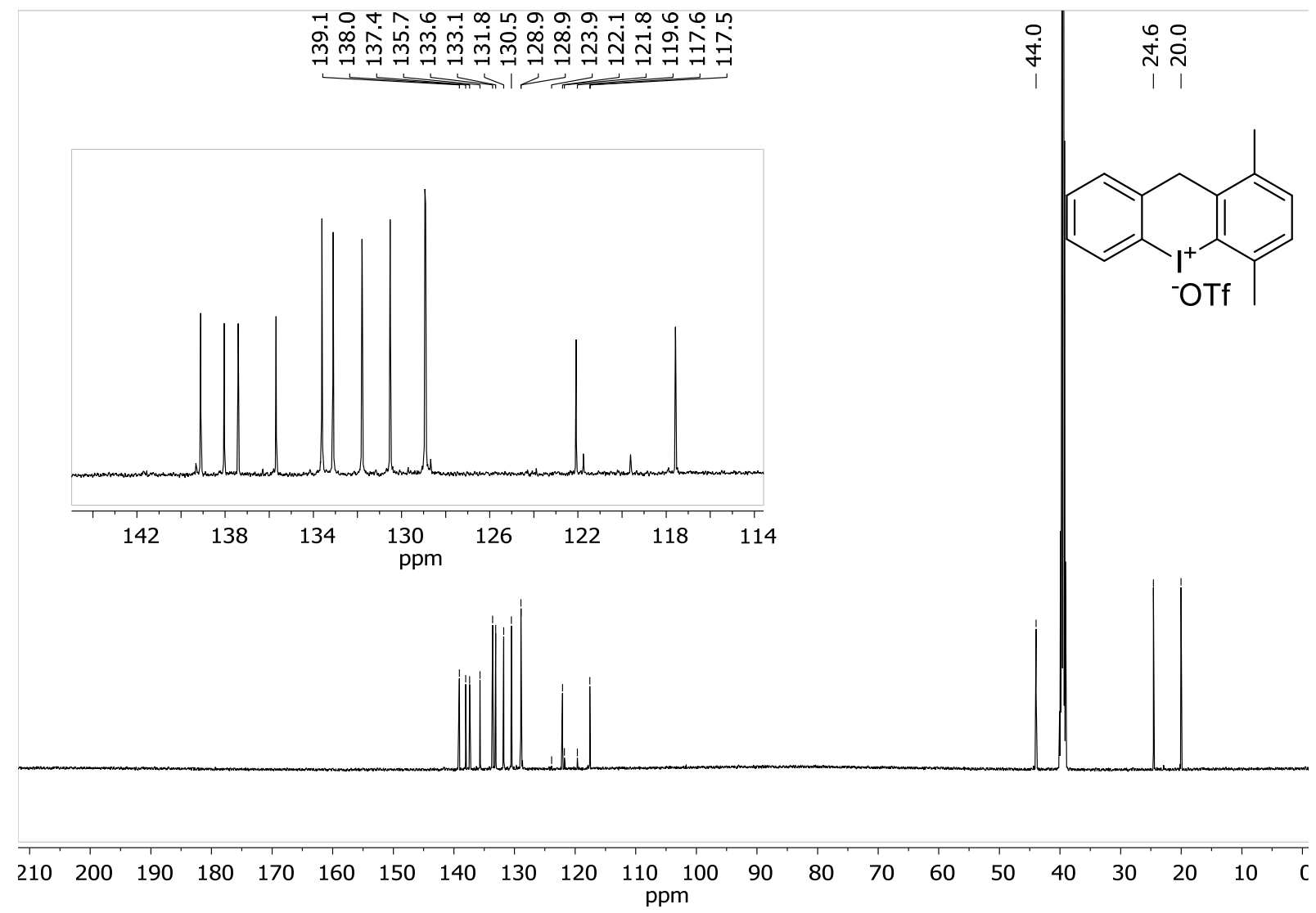

Figure S53: $151 \mathrm{MHz}{ }^{13} \mathrm{C}-\mathrm{NMR}$ spectrum of compound $\mathbf{7 n}$. 


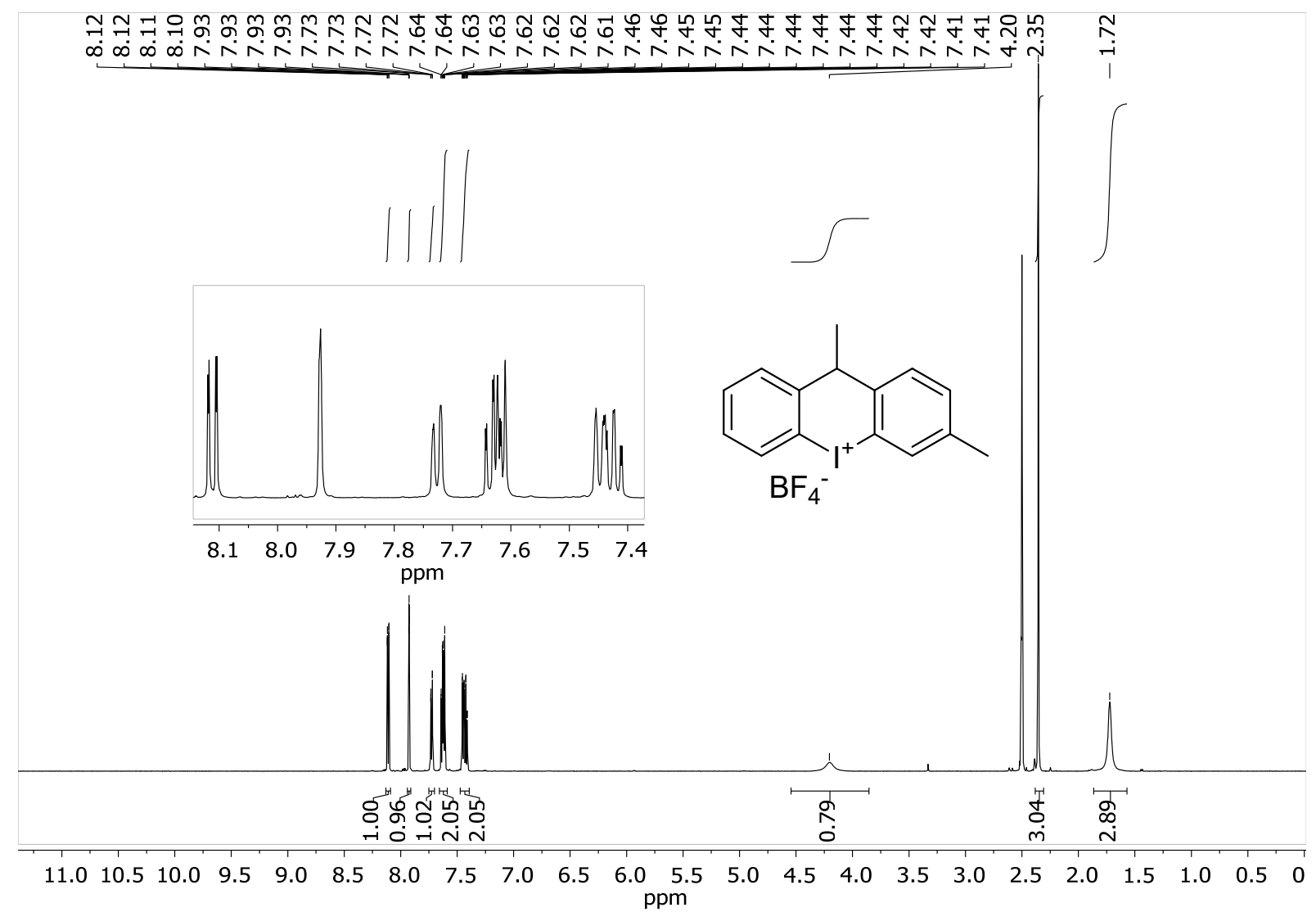

Figure S54: $600 \mathrm{MHz}{ }^{1} \mathrm{H}-\mathrm{NMR}$ spectrum of compound 8 a.

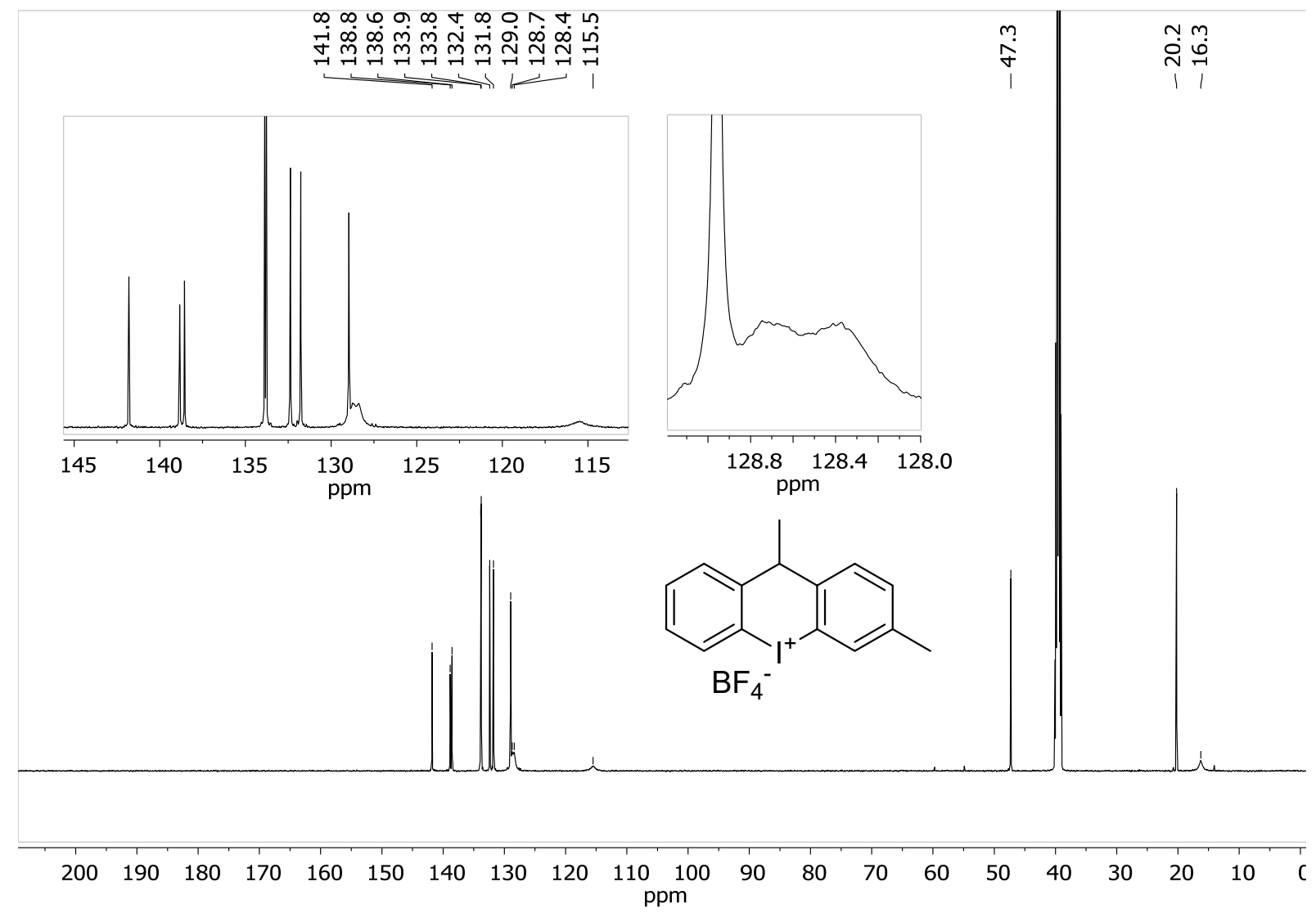

Figure S55: $151 \mathrm{MHz}{ }^{13} \mathrm{C}-\mathrm{NMR}$ spectrum of compound 8 . 


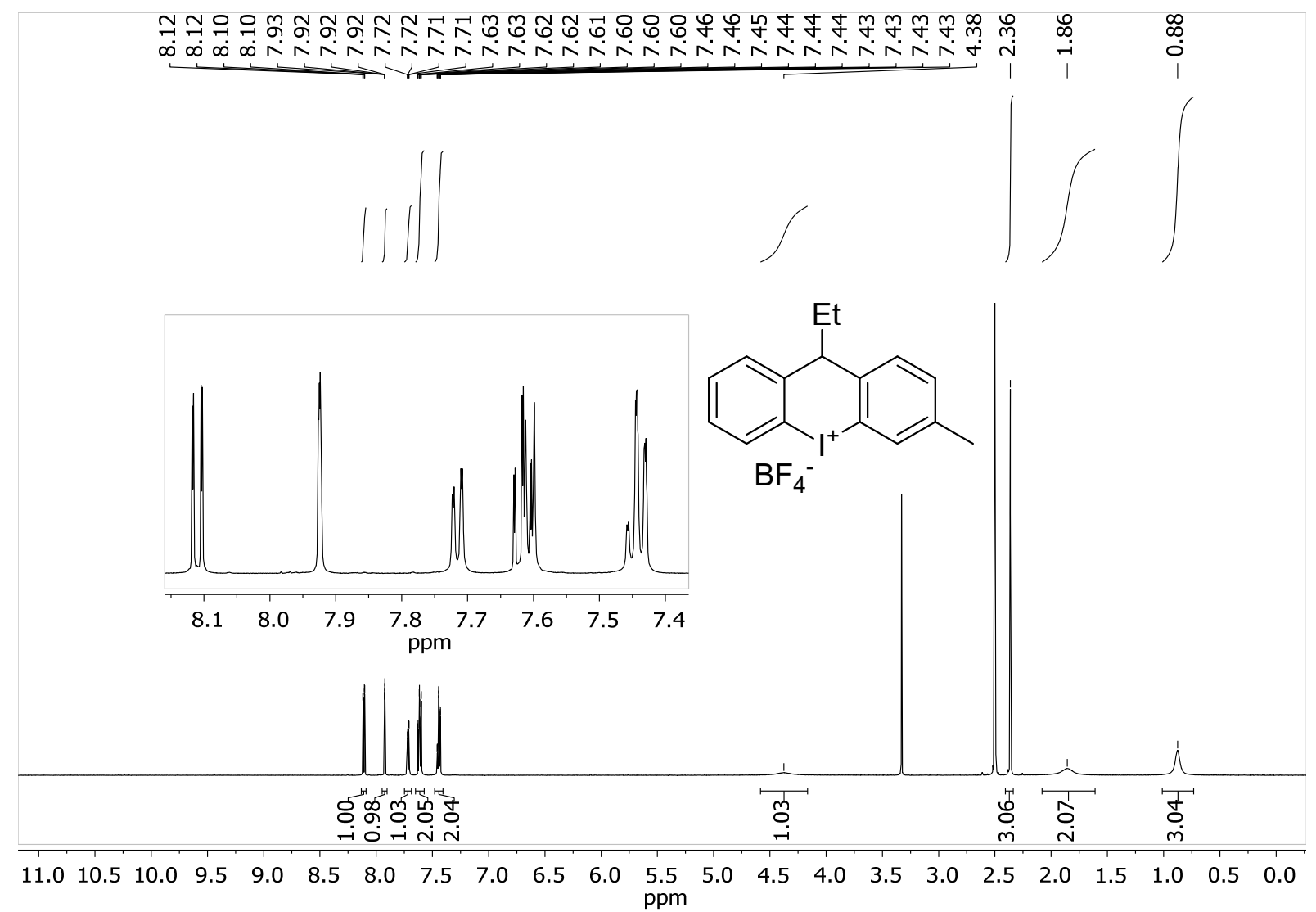

Figure S56: $600 \mathrm{MHz}{ }^{1} \mathrm{H}-\mathrm{NMR}$ spectrum of compound $8 \boldsymbol{b}$.

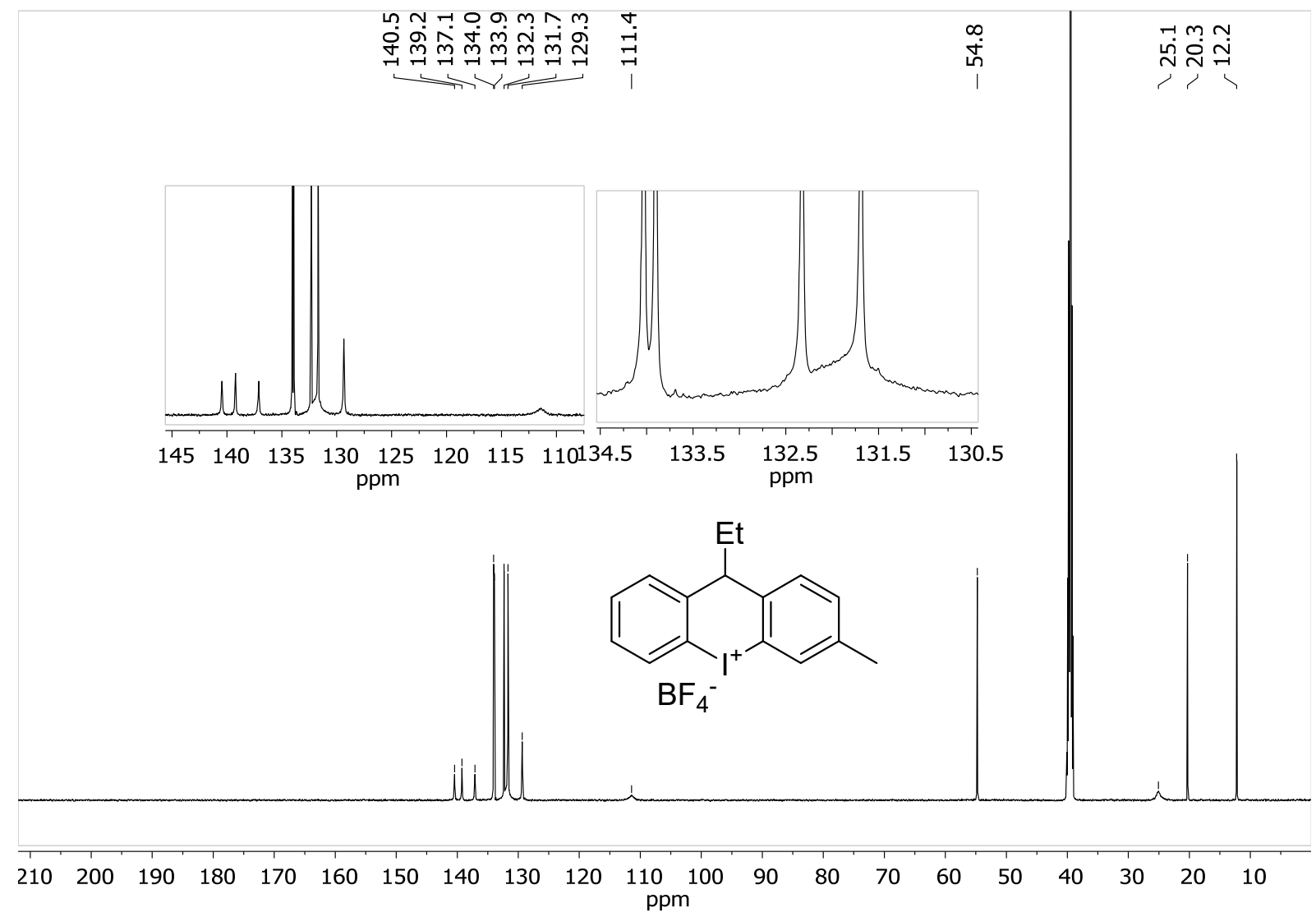

Figure S57: $151 \mathrm{MHz}{ }^{13} \mathrm{C}-N M R$ spectrum of compound $8 \mathbf{b}$. 


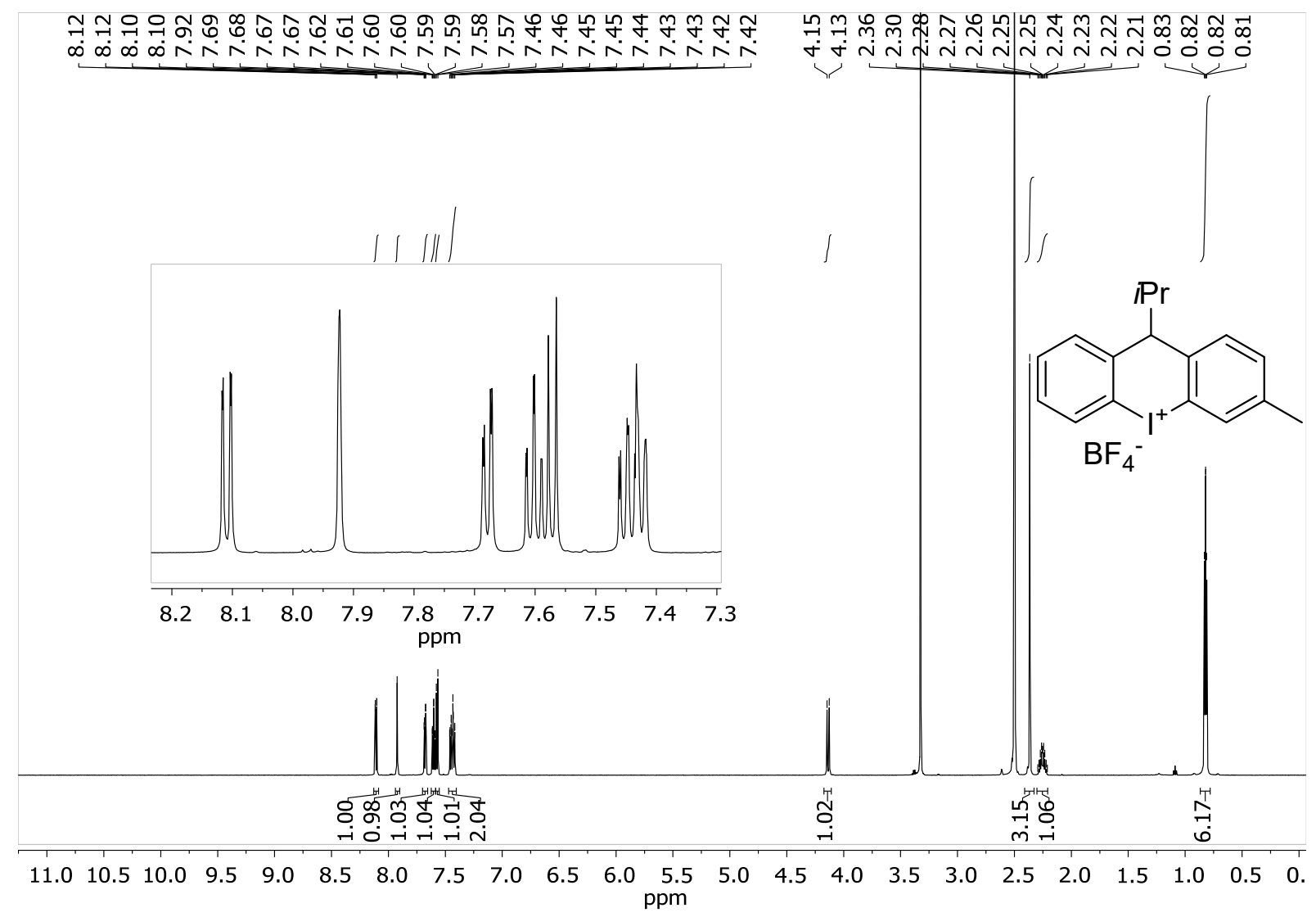

Figure S58: $600 \mathrm{MHz}{ }^{1} \mathrm{H}-\mathrm{NMR}$ spectrum of compound $8 \mathrm{c}$.

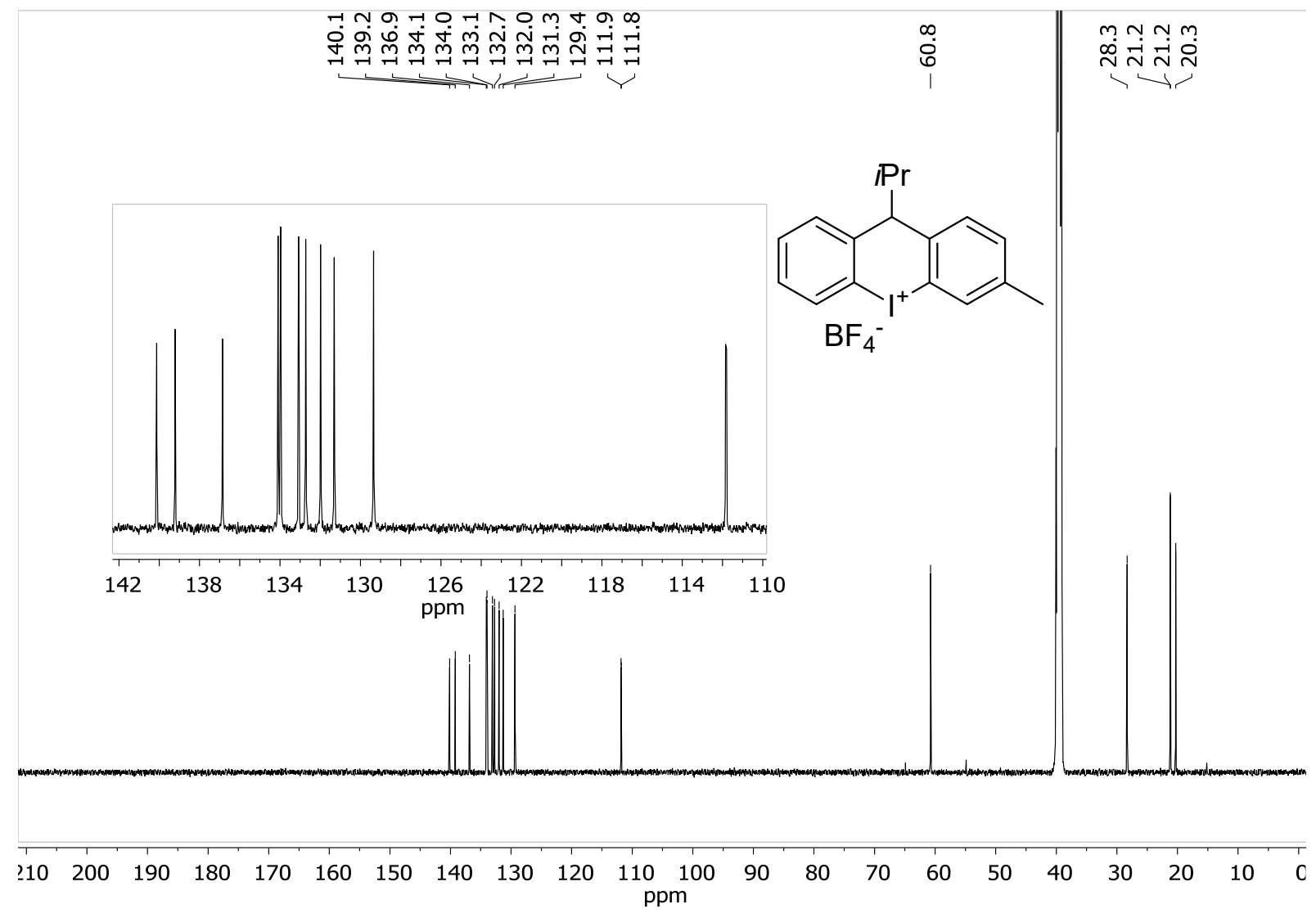

Figure S59: $151 \mathrm{MHz}{ }^{13} \mathrm{C}-\mathrm{NMR}$ spectrum of compound $8 \mathrm{c}$. 


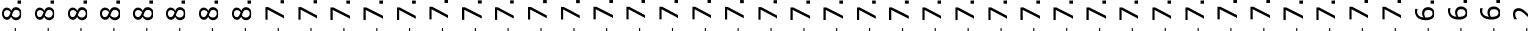
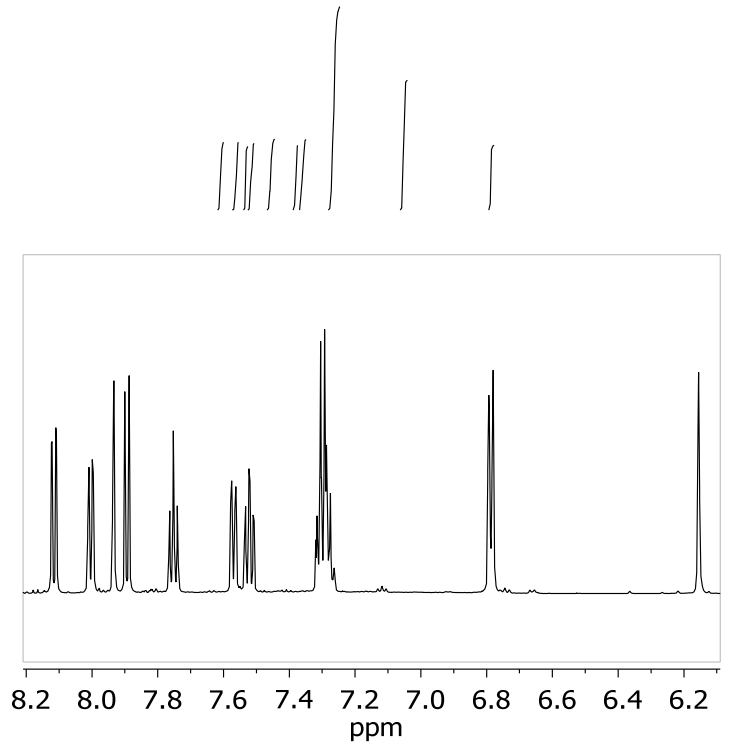<smiles>Cc1ccc2c(c1)[I+]c1ccccc1C2c1ccccc1</smiles>

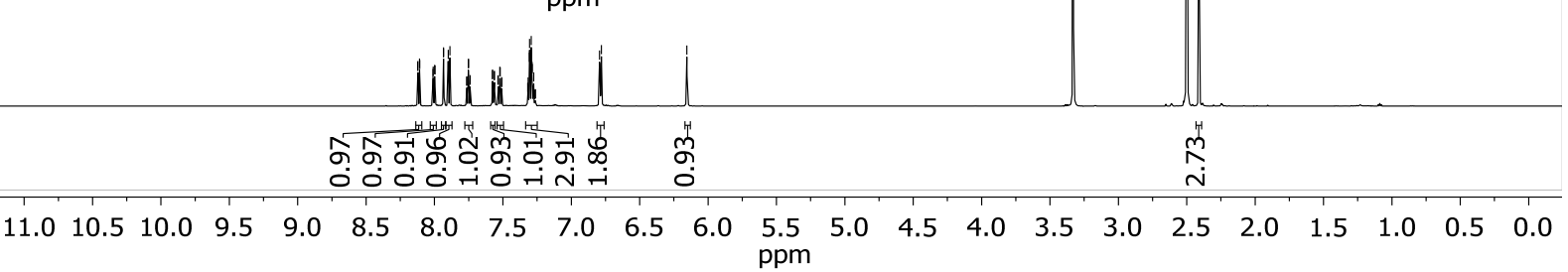

Figure S60: $600 \mathrm{MHz}^{1} \mathrm{H}-\mathrm{NMR}$ spectrum of compound $8 d$.

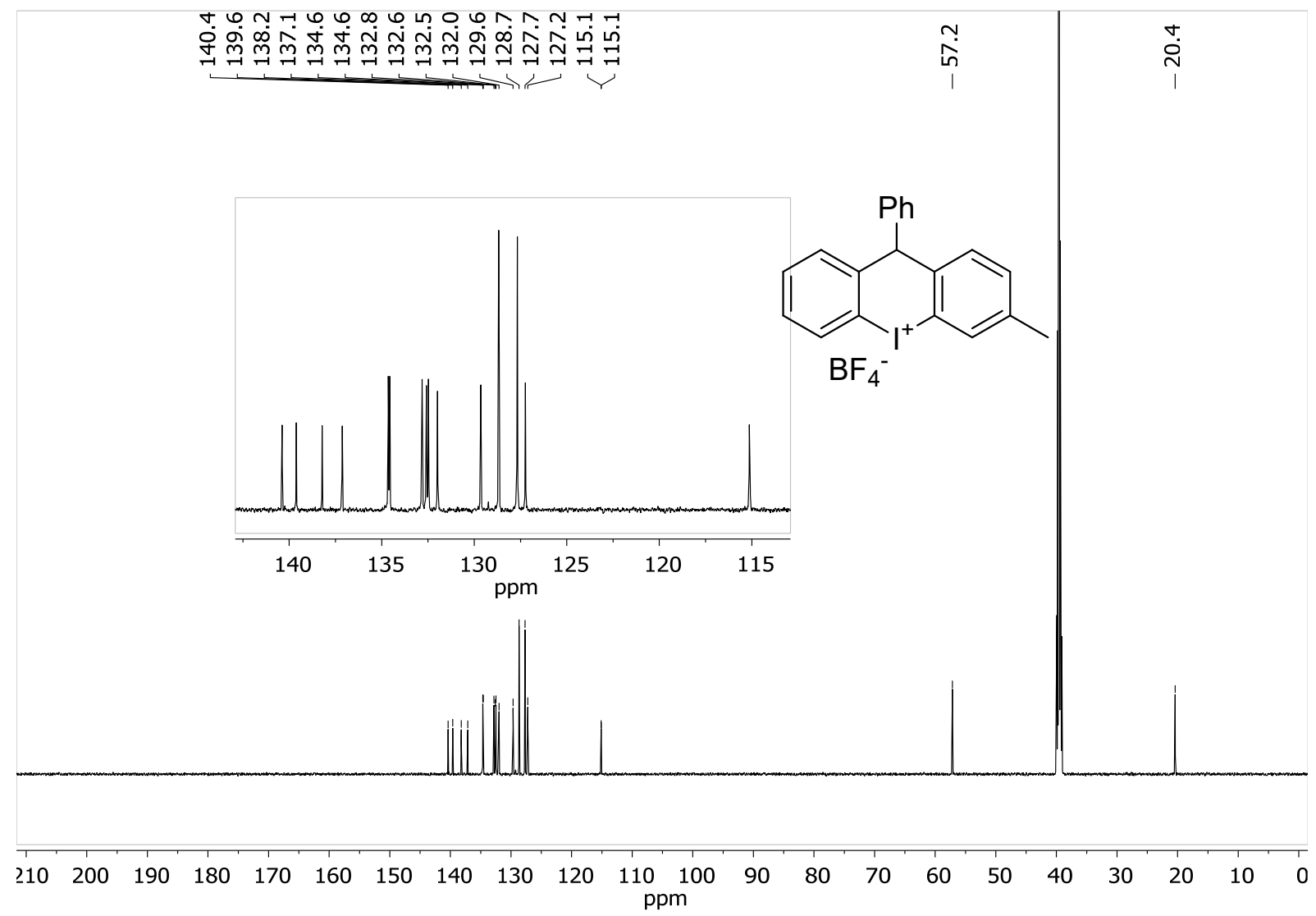

Figure S61: $151 \mathrm{MHz}{ }^{13} \mathrm{C}-\mathrm{NMR}$ spectrum of compound 8 . 


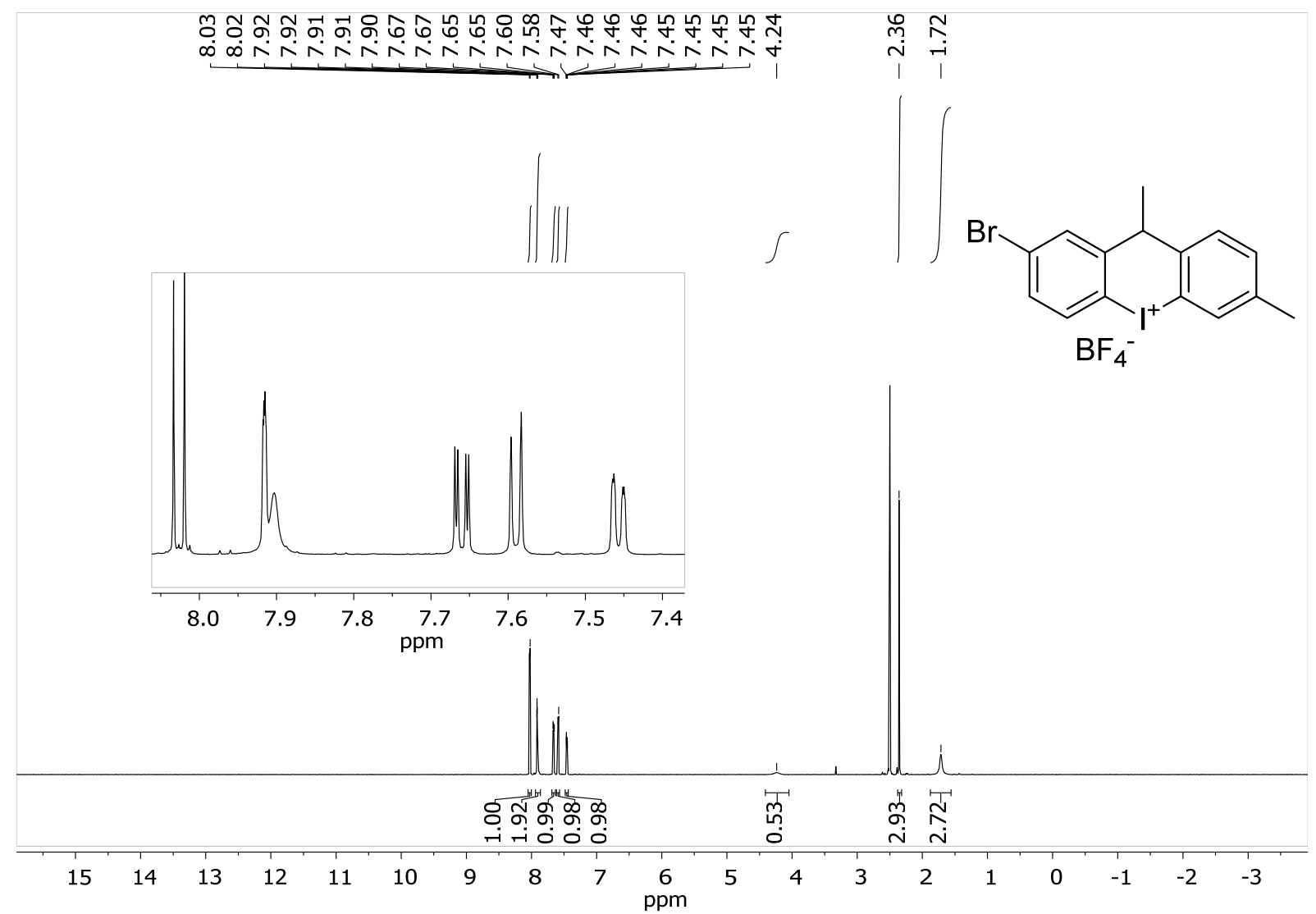

Figure S62: $600 \mathrm{MHz}{ }^{1} \mathrm{H}-\mathrm{NMR}$ spectrum of compound 8 .

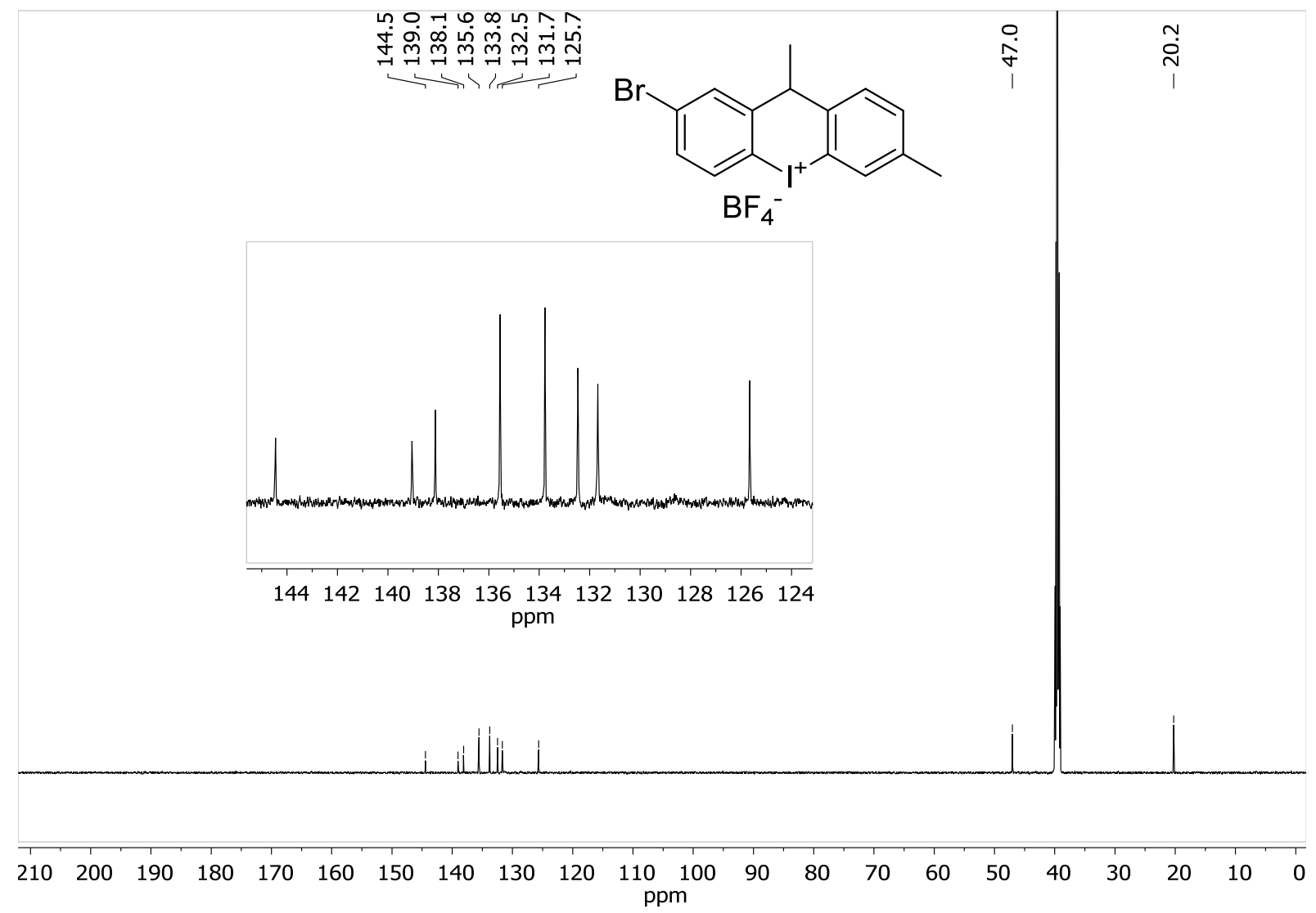

Figure S63: $151 \mathrm{MHz}{ }^{13} \mathrm{C}-\mathrm{NMR}$ spectrum of compound $8 \boldsymbol{e}$. 


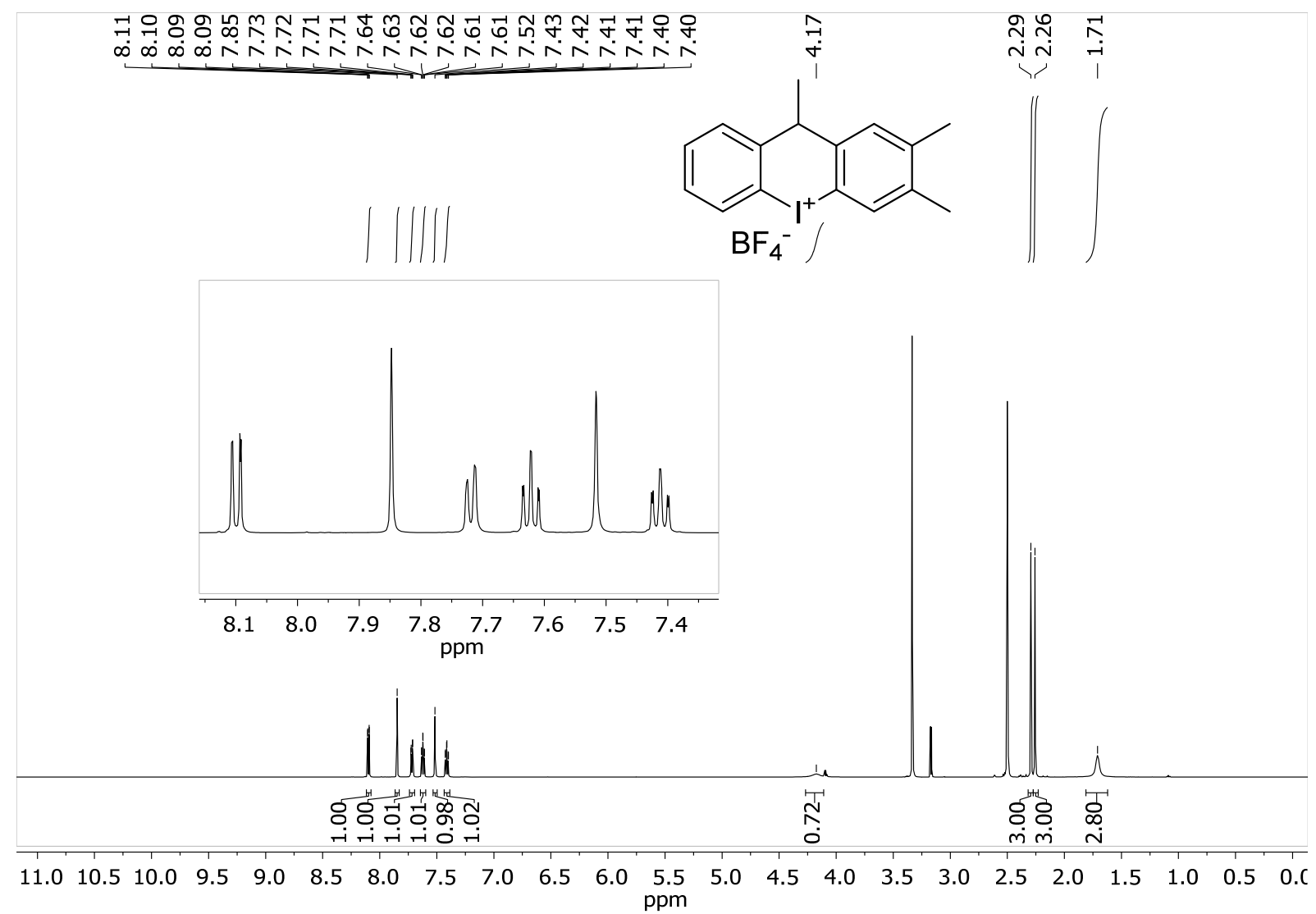

Figure S64: $600 \mathrm{MHz}{ }^{1} \mathrm{H}-\mathrm{NMR}$ spectrum of compound $8 f$.

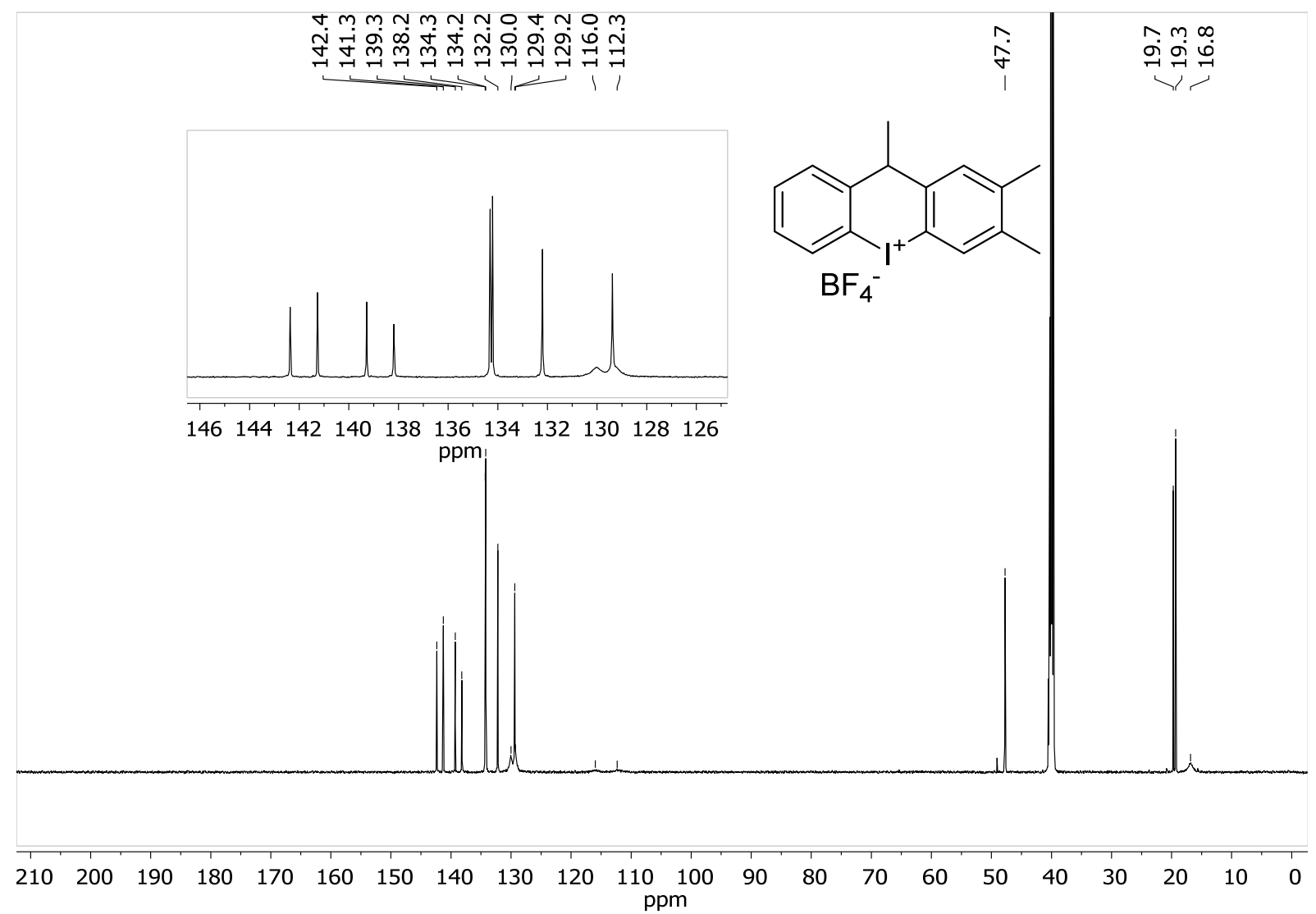

Figure S65: $151 \mathrm{MHz}{ }^{13} \mathrm{C}-\mathrm{NMR}$ spectrum of compound 8f. 


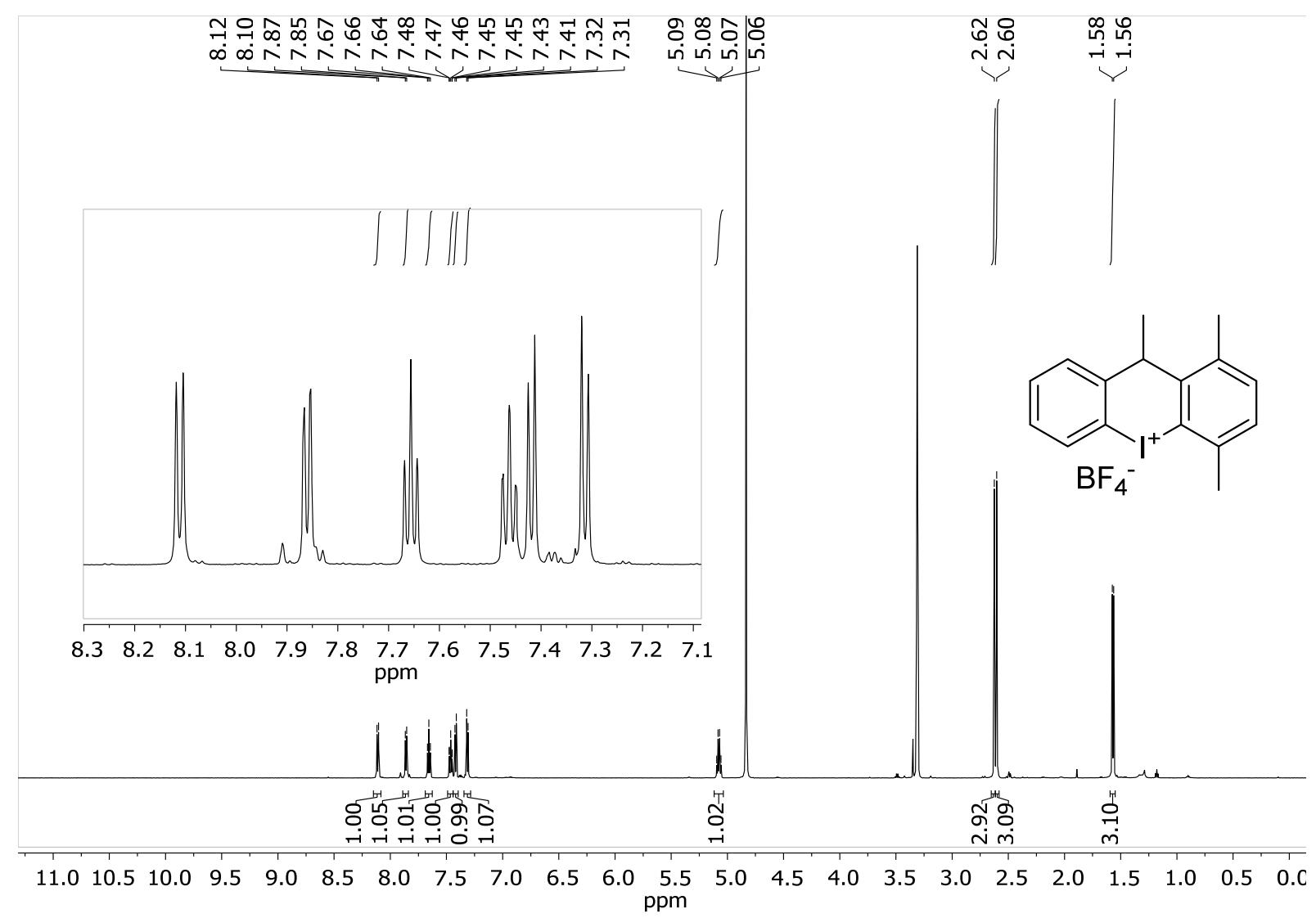

Figure S66: $600 \mathrm{MHz}{ }^{1} \mathrm{H}-\mathrm{NMR}$ spectrum of compound $8 \mathrm{~g}$.

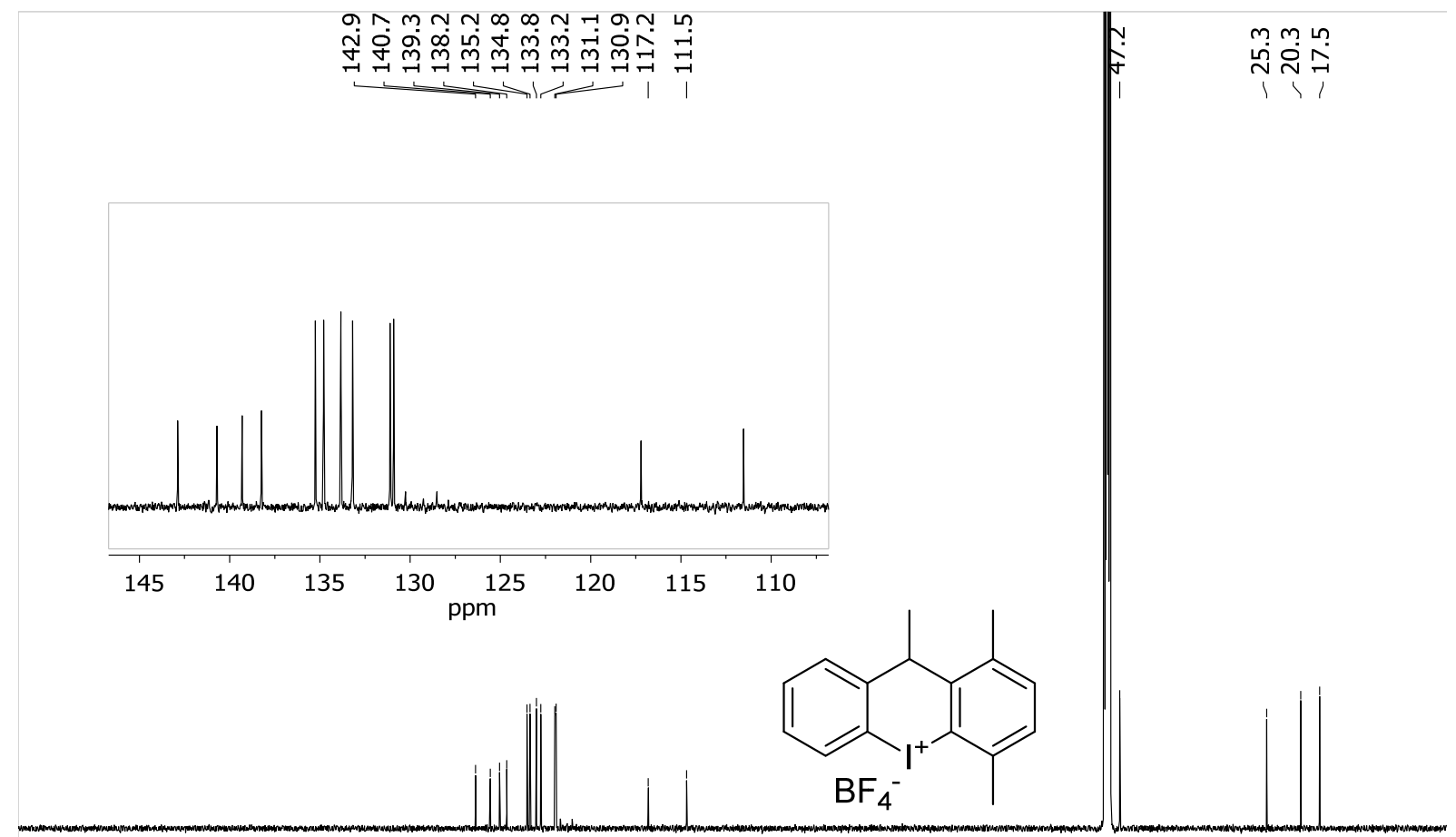

$\begin{array}{llllllllllllllllllllll}10 & 200 & 190 & 180 & 170 & 160 & 150 & 140 & 130 & 120 & \begin{array}{c}110 \\ \mathrm{ppm}\end{array} & 100 & 90 & 80 & 70 & 60 & 50 & 40 & 30 & 20 & 10 & 0\end{array}$

Figure S67: $151 \mathrm{MHz}{ }^{13} \mathrm{C}-\mathrm{NMR}$ spectrum of compound $8 \mathrm{~g}$. 


\section{Crystallographic Data}

\section{Crystallographic data for compound $7 \mathbf{k}$}

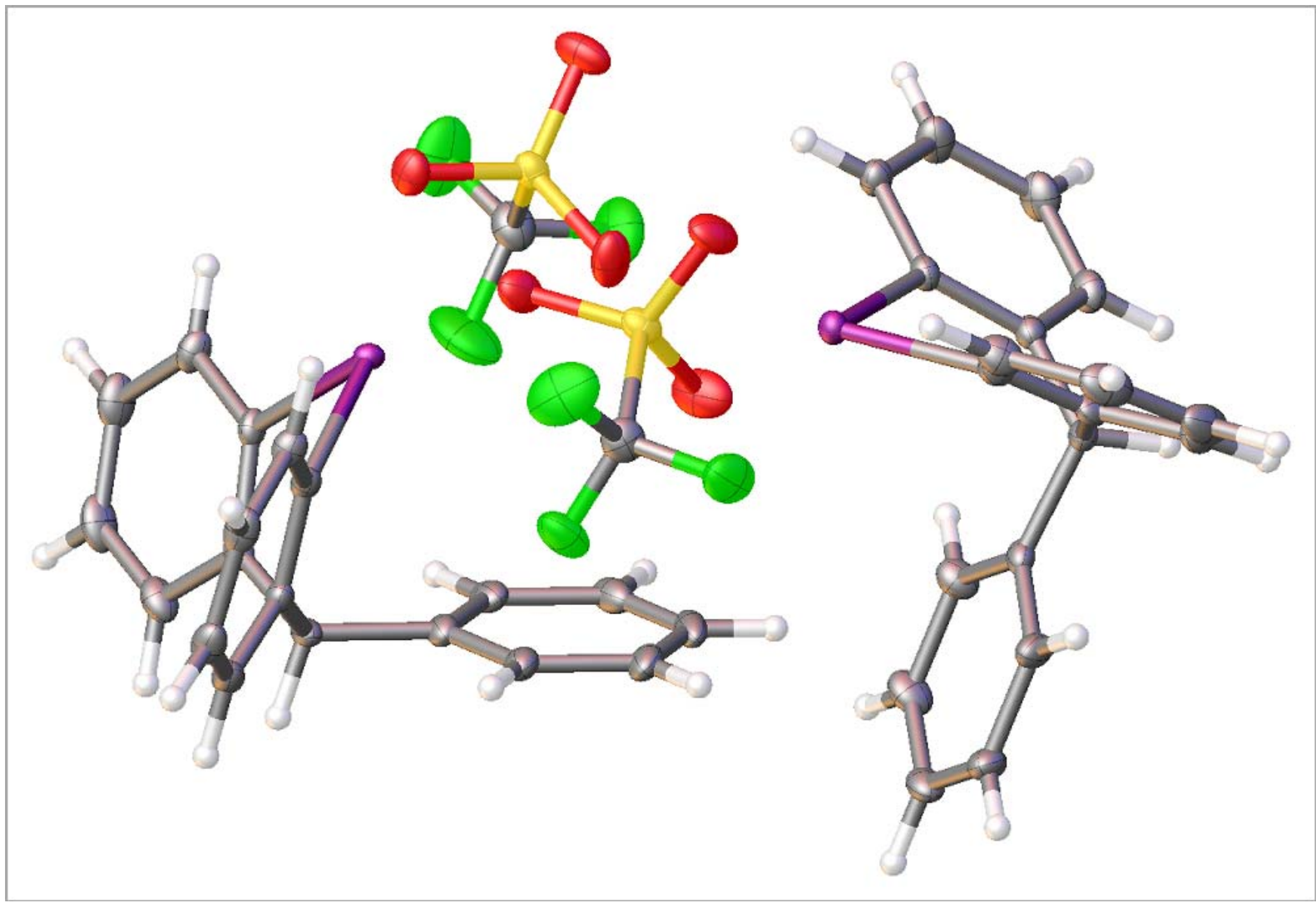

Table S9: Crystal data and structure refinement for $\mathbf{7 k}$.

\begin{tabular}{|c|c|}
\hline Empirical formula & $\mathrm{C}_{20} \mathrm{H}_{14} \mathrm{~F}_{3} \mathrm{IO}_{3} \mathrm{~S}$ \\
\hline Formula weight & 518.27 \\
\hline Temperature/K & 100.0 \\
\hline Crystal system & orthorhombic \\
\hline Space group & $\mathrm{P} 2{ }_{1} 2_{1} 2_{1}$ \\
\hline $\mathrm{a} / \AA ̊ \AA$ & $13.6314(4)$ \\
\hline b/Å & $19.2653(5)$ \\
\hline$c / \AA$ & $14.8884(4)$ \\
\hline$\alpha /^{\circ}$ & 90 \\
\hline$\beta /^{\circ}$ & 90 \\
\hline$v / /^{\circ}$ & 90 \\
\hline Volume $/ \AA^{3}$ & $3909.89(19)$ \\
\hline $\mathbf{Z}$ & 8 \\
\hline$\rho_{\text {calcg }} / \mathrm{cm}^{3}$ & 1.761 \\
\hline$\mu / \mathrm{mm}^{-1}$ & 1.791 \\
\hline$F(000)$ & 2032.0 \\
\hline Crystal size $/ \mathrm{mm}^{3}$ & $0.2 \times 0.2 \times 0.15$ \\
\hline Radiation & $\operatorname{MoK} \alpha(\lambda=0.71073)$ \\
\hline 20 range for data collection $/^{\circ}$ & 4.57 to 72.828 \\
\hline Index ranges & $-22 \leq h \leq 22,-32 \leq k \leq 31,-17 \leq 1 \leq 24$ \\
\hline Reflections collected & 91480 \\
\hline Independent reflections & $19037\left[R_{\text {int }}=0.0351, R_{\text {sigma }}=0.0334\right]$ \\
\hline Data/restraints/parameters & $19037 / 0 / 506$ \\
\hline
\end{tabular}




\begin{tabular}{|c|c|}
\hline Goodness-of-fit on $F^{2}$ & 1.029 \\
\hline Final $R$ indexes $[I>=2 \sigma(I)]$ & $R_{1}=0.0266, w R_{2}=0.0497$ \\
\hline Final $R$ indexes [all data] & $R_{1}=0.0372, w R_{2}=0.0530$ \\
\hline Largest diff. peak/hole / e $\AA^{-3}$ & $1.07 /-0.75$ \\
\hline Flack parameter & $0.391(10)$ \\
\hline
\end{tabular}

Table S10: Fractional Atomic Coordinates $\left(\times 10^{4}\right)$ and Equivalent Isotropic Displacement Parameters $\left(\AA^{2} \times 10^{3}\right)$ for $7 \mathbf{k} . U_{e q}$ is defined as $1 / 3$ of of the trace of the orthogonalised $U_{I J}$ tensor.

\begin{tabular}{|c|c|c|c|c|}
\hline Atom & $x$ & $y$ & $z$ & $U(e q)$ \\
\hline I1 & $2955.8(2)$ & $2530.2(2)$ & $5226.5(2)$ & $10.97(3)$ \\
\hline 1002 & $4015.6(2)$ & $2542.9(2)$ & $8613.3(2)$ & $14.30(3)$ \\
\hline S21 & $2603.8(5)$ & $3949.8(3)$ & $6917.7(5)$ & $17.81(12)$ \\
\hline F21 & 2893(2) & $5233.1(10)$ & $6414.8(17)$ & $51.0(6)$ \\
\hline F22 & $4084(2)$ & $4548.1(14)$ & $6120.7(18)$ & $56.1(7)$ \\
\hline F23 & $3830.7(18)$ & $4895.7(11)$ & $7478.9(15)$ & $42.8(6)$ \\
\hline 021 & $3285(2)$ & $3432.6(11)$ & $7245.4(16)$ & $32.5(5)$ \\
\hline 022 & $1903.5(18)$ & $4191.2(12)$ & $7568.7(16)$ & $31.1(5)$ \\
\hline 023 & $2201.9(16)$ & $3795.8(10)$ & $6040.5(14)$ & $24.5(4)$ \\
\hline COAA & $4018(2)$ & $1190.8(13)$ & $9635(2)$ & $23.5(5)$ \\
\hline C1 & $4525(2)$ & $1200.7(14)$ & $3391.7(18)$ & $19.7(5)$ \\
\hline C2 & $3903(2)$ & $635.5(14)$ & $3287.9(19)$ & $22.5(5)$ \\
\hline C3 & $3042(2)$ & $584.5(13)$ & $3771.8(18)$ & $21.0(5)$ \\
\hline C4 & $2767(2)$ & $1119.5(12)$ & $4342.9(18)$ & $17.0(5)$ \\
\hline C4A & $3389.7(19)$ & $1684.1(12)$ & $4423.2(18)$ & $13.8(4)$ \\
\hline C5 & $3090(2)$ & $3835.9(12)$ & $4089.3(18)$ & $19.2(5)$ \\
\hline C5A & $3572(2)$ & $3221.5(12)$ & $4286.0(17)$ & $14.4(5)$ \\
\hline C6 & $3511(3)$ & $4273.9(14)$ & $3457(2)$ & $25.8(6)$ \\
\hline C7 & $4377(3)$ & $4093.4(15)$ & $3043(2)$ & $26.9(6)$ \\
\hline C8 & $4854(2)$ & $3471.8(15)$ & $3252.5(18)$ & $22.6(5)$ \\
\hline C8A & $4457.6(19)$ & $3023.7(13)$ & $3893.1(17)$ & $15.8(4)$ \\
\hline C9 & $4961.2(17)$ & $2347.7(12)$ & $4144.9(15)$ & $15.4(4)$ \\
\hline C9A & $4283.6(19)$ & $1739.1(13)$ & $3982.5(17)$ & $14.8(4)$ \\
\hline C10 & $5324.7(17)$ & $2355.1(13)$ & $5123.0(16)$ & $17.5(5)$ \\
\hline C11 & $5500(2)$ & $1726.6(16)$ & $5563.5(19)$ & $20.5(5)$ \\
\hline C11' & $5492(2)$ & $2973.8(16)$ & $5578(2)$ & $21.1(5)$ \\
\hline C12 & $5831(2)$ & $1720.7(18)$ & $6450(2)$ & $25.5(6)$ \\
\hline C12' & $5825(2)$ & $2964.9(18)$ & $6466(2)$ & $26.1(6)$ \\
\hline C13 & $5994.1(19)$ & $2340.3(18)$ & $6897.0(18)$ & $29.0(7)$ \\
\hline C21 & $5142(2)$ & $3947.0(14)$ & $10627.8(19)$ & $21.2(5)$ \\
\hline C22 & $4523(2)$ & $4519.4(15)$ & $10543(2)$ & $25.4(6)$ \\
\hline C23 & $3767(2)$ & $4528.0(14)$ & $9923.7(19)$ & $23.5(5)$ \\
\hline C24 & $3623(2)$ & $3957.3(13)$ & $9367.2(17)$ & $17.4(4)$ \\
\hline C24A & $4258.2(19)$ & $3396.9(13)$ & $9458.7(17)$ & $15.2(4)$ \\
\hline C25A & $4451(2)$ & $1843.4(13)$ & $9629.2(18)$ & $18.0(5)$ \\
\hline C26 & $4320(2)$ & $726.5(15)$ & $10293(2)$ & $29.9(7)$ \\
\hline C27 & $5016(3)$ & $920.7(16)$ & $10922(2)$ & $29.8(7)$ \\
\hline C28 & $5422(2)$ & $1579.8(15)$ & $10907.1(19)$ & $23.3(5)$ \\
\hline C28A & $5161.9(18)$ & $2058.9(13)$ & $10235.8(18)$ & $17.5(4)$ \\
\hline C29 & $5699.0(18)$ & $2746.0(13)$ & $10174.5(17)$ & $16.6(4)$ \\
\hline C29A & $5023.3(18)$ & $3366.3(13)$ & $10076.7(16)$ & $16.0(4)$ \\
\hline C30 & $6509.9(18)$ & $2708.3(13)$ & $9448.3(16)$ & $16.4(4)$ \\
\hline
\end{tabular}




\begin{tabular}{lllll}
\hline C31 & $6817(2)$ & $3301.9(14)$ & $8996.0(19)$ & $21.8(5)$ \\
\hline C31' & $7000(2)$ & $2086.7(15)$ & $9284.8(18)$ & $21.7(5)$ \\
C32 & $7607(2)$ & $3270.3(16)$ & $8398(2)$ & $25.5(6)$ \\
\hline C32' & $7791(2)$ & $2061.4(15)$ & $8690.4(19)$ & $23.1(5)$ \\
C33 & $8092.6(19)$ & $2652.5(15)$ & $8249.3(17)$ & $22.5(5)$ \\
C34 & $3393(3)$ & $4698.5(17)$ & $6722(2)$ & $27.5(7)$ \\
S1 & $2752.5(5)$ & $1141.1(3)$ & $7135.5(5)$ & $18.84(13)$ \\
F1 & $2491.0(18)$ & $-45.0(11)$ & $6329.0(19)$ & $48.1(6)$ \\
F2 & $3635.4(17)$ & $-59.0(10)$ & $7319.5(15)$ & $38.0(5)$ \\
F3 & $3890.8(18)$ & $432.3(10)$ & $6048.8(14)$ & $34.2(5)$ \\
O1 & $3647.3(18)$ & $1472.2(12)$ & $7441.6(18)$ & $36.1(6)$ \\
O2 & $2091.8(18)$ & $941.0(11)$ & $7837.6(15)$ & $30.6(5)$ \\
O3 & $2297.6(17)$ & $1469.9(10)$ & $6371.8(15)$ & $26.7(4)$ \\
C14 & $3225(3)$ & $326.2(15)$ & $6683(2)$ & $24.0(6)$ \\
\hline
\end{tabular}

Table S11: Anisotropic Displacement Parameters $\left(\AA^{2} \times 10^{3}\right)$ for $\mathbf{7 k}$. The Anisotropic displacement factor exponent takes the form: $-2 \pi^{2}\left[h^{2} a^{* 2} U_{11}+2 h k a^{*} b^{*} U_{12}+\ldots\right]$.

\begin{tabular}{lllllll}
\hline Atom & $U_{11}$ & $U_{22}$ & $U_{33}$ & $U_{23}$ & $U_{13}$ & $U_{12}$ \\
I1 & $10.04(5)$ & $9.58(5)$ & $13.30(5)$ & $-0.60(6)$ & $1.36(4)$ & $0.13(5)$ \\
I002 & $16.35(6)$ & $14.15(6)$ & $12.41(5)$ & $-0.88(6)$ & $-1.07(4)$ & $0.07(6)$ \\
S21 & $24.1(3)$ & $13.5(2)$ & $15.9(3)$ & $-2.7(2)$ & $1.2(2)$ & $0.6(2)$ \\
F21 & $77.7(18)$ & $18.8(8)$ & $56.6(14)$ & $13.0(9)$ & - & $-4.7(10)$ \\
F22 & $50.8(16)$ & $66.7(16)$ & $50.9(15)$ & $-8.3(12)$ & $28.3(13)$ & - \\
& & & & & & $21.9(13)$ \\
F23 & $46.7(13)$ & $43.5(12)$ & $38.0(12)$ & $-5.1(10)$ & - & - \\
& & & & & $12.5(10)$ & $21.0(10)$ \\
O21 & $50.9(15)$ & $21.7(10)$ & $24.7(11)$ & $1.1(8)$ & $-8.4(10)$ & $12.5(10)$ \\
O22 & $30.0(12)$ & $32.6(11)$ & $30.8(12)$ & $-14.5(9)$ & $11.3(10)$ & $-6.2(9)$ \\
O23 & $31.2(11)$ & $21.6(9)$ & $20.7(9)$ & $-6.5(7)$ & $-5.8(8)$ & $1.9(8)$ \\
C0AA & $25.7(13)$ & $18.2(11)$ & $26.6(14)$ & $1.5(10)$ & $6.3(11)$ & $-3.3(10)$ \\
C1 & $19.7(12)$ & $24.2(12)$ & $15.1(11)$ & $-3.4(9)$ & $-1.8(9)$ & $8.5(10)$ \\
C2 & $28.1(14)$ & $19.8(11)$ & $19.5(13)$ & $-7.0(9)$ & $-6.5(11)$ & $8.3(10)$ \\
C3 & $28.7(14)$ & $11.9(9)$ & $22.3(13)$ & $-3.6(8)$ & $-8.3(11)$ & $2.0(9)$ \\
C4 & $20.0(12)$ & $12.0(9)$ & $19.1(11)$ & $-0.9(8)$ & $-3.3(9)$ & $0.1(8)$ \\
C4A & $15.4(11)$ & $11.9(9)$ & $14.0(11)$ & $-1.6(8)$ & $-2.2(9)$ & $3.0(8)$ \\
C5 & $23.3(13)$ & $13.1(9)$ & $21.0(12)$ & $1.0(9)$ & $-6.4(10)$ & $0.1(9)$ \\
C5A & $18.2(12)$ & $11.9(9)$ & $13.3(11)$ & $2.9(8)$ & $-3.7(9)$ & $-2.8(8)$ \\
C6 & $38.3(17)$ & $15.3(11)$ & $23.8(14)$ & $5.0(10)$ & $-7.5(13)$ & $-5.3(11)$ \\
C7 & $40.9(17)$ & $20.9(12)$ & $18.9(13)$ & $6.8(10)$ & $-2.4(12)$ & - \\
& & & & & & $12.0(12)$ \\
C8 & $25.4(13)$ & $25.3(13)$ & $17.0(12)$ & $1.0(10)$ & $2.4(10)$ & - \\
C8A & $15.5(11)$ & $19.0(11)$ & $12.8(11)$ & $-0.1(8)$ & $0.1(8)$ & $-5.5(9)$ \\
C9 & $11.3(9)$ & $22.1(11)$ & $12.9(9)$ & $-1.7(8)$ & $0.8(7)$ & $-0.1(8)$ \\
C9A & $14.9(10)$ & $16.8(10)$ & $12.6(10)$ & $-1.0(8)$ & $-2.1(8)$ & $3.2(8)$ \\
C10 & $11.1(9)$ & $26.6(13)$ & $14.7(10)$ & $-0.1(9)$ & $-1.9(7)$ & $-1.2(8)$ \\
C11 & $12.9(11)$ & $30.5(14)$ & $18.2(12)$ & $2.3(11)$ & $-1.1(9)$ & $0.8(10)$ \\
C11' & $11.9(11)$ & $30.5(14)$ & $20.8(13)$ & $-4.6(11)$ & $-1.8(9)$ & $0.2(10)$ \\
C12 & $14.0(12)$ & $42.4(16)$ & $20.0(13)$ & $5.0(12)$ & $-3.5(10)$ & $2.0(11)$ \\
C12' & $15.5(12)$ & $42.6(16)$ & $20.1(13)$ & - & $-0.6(10)$ & $-2.6(11)$ \\
& & & & $11.7(13)$ & & \\
\hline & & & & & & \\
\hline
\end{tabular}




\begin{tabular}{lllllll}
\hline C13 & $13.4(10)$ & $59(2)$ & $14.7(10)$ & $-1.1(12)$ & $-1.2(9)$ & $-2.7(12)$ \\
C21 & $22.6(13)$ & $23.5(12)$ & $17.3(12)$ & $-3.2(10)$ & $-2.8(10)$ & $-5.2(10)$ \\
C22 & $34.8(16)$ & $19.7(12)$ & $21.7(13)$ & $-5.2(10)$ & $-3.1(12)$ & $-2.8(11)$ \\
C23 & $34.0(15)$ & $15.3(10)$ & $21.3(13)$ & $-1.3(9)$ & $-2.2(11)$ & $2.9(10)$ \\
C24 & $20.4(12)$ & $16.1(10)$ & $15.7(11)$ & $0.6(8)$ & $-1.5(9)$ & $2.3(9)$ \\
C24A & $17.6(11)$ & $16.4(10)$ & $11.7(10)$ & $-0.6(8)$ & $-0.3(8)$ & $-2.0(8)$ \\
C25A & $19.3(12)$ & $17.0(10)$ & $17.7(12)$ & $3.8(9)$ & $3.8(9)$ & $2.2(9)$ \\
C26 & $34.4(16)$ & $19.7(12)$ & $35.5(17)$ & $8.4(12)$ & $13.2(14)$ & $1.6(11)$ \\
C27 & $34.4(16)$ & $26.4(13)$ & $28.4(10)$ & $15.1(12)$ & $11.9(13)$ & $11.1(12)$ \\
C28 & $22.9(13)$ & $27.6(13)$ & $19.6(12)$ & $9.4(10)$ & $4.4(10)$ & $6.1(10)$ \\
C28A & $17.1(11)$ & $20.5(10)$ & $14.9(10)$ & $4.7(9)$ & $4.1(9)$ & $1.8(8)$ \\
C29 & $14.1(10)$ & $22.6(10)$ & $13.1(10)$ & $1.4(9)$ & $-0.8(8)$ & $1.4(8)$ \\
C29A & $15.3(10)$ & $19.3(10)$ & $13.3(10)$ & $0.6(8)$ & $0.1(8)$ & $-1.0(8)$ \\
C30 & $13.8(10)$ & $22.9(11)$ & $12.6(10)$ & $2.1(8)$ & $-2.3(8)$ & $-1.9(8)$ \\
C31 & $24.0(13)$ & $19.6(11)$ & $21.9(13)$ & $0.0(10)$ & $4.9(10)$ & $-4.5(9)$ \\
C31' & $17.1(11)$ & $26.5(12)$ & $21.5(12)$ & $8.4(10)$ & $1.6(10)$ & $4.2(10)$ \\
C32 & $27.8(14)$ & $26.2(13)$ & $22.6(13)$ & $0.5(10)$ & $7.2(11)$ & $-8.8(11)$ \\
C32' & $16.0(12)$ & $30.6(13)$ & $22.7(13)$ & $4.1(11)$ & $1.8(10)$ & $5.8(10)$ \\
C33 & $14.4(10)$ & $34.7(15)$ & $18.4(11)$ & $0.4(10)$ & $3.2(8)$ & $-3.6(9)$ \\
C34 & $32.6(17)$ & $24.3(13)$ & $25.6(15)$ & $1.2(11)$ & $1.1(13)$ & $-6.7(12)$ \\
S1 & $22.1(3)$ & $15.1(2)$ & $19.3(3)$ & $-0.3(2)$ & $5.7(2)$ & $-1.2(2)$ \\
F1 & $45.2(13)$ & $25.0(9)$ & $74.2(17)$ & - & - & $-8.1(9)$ \\
& & & & $18.1(11)$ & $13.0(13)$ & \\
F2 & $44.5(13)$ & $31.1(10)$ & $38.3(12)$ & $12.0(8)$ & $5.5(10)$ & $15.1(9)$ \\
F3 & $44.8(12)$ & $29.3(9)$ & $28.4(10)$ & $-1.8(8)$ & $13.8(9)$ & $8.4(9)$ \\
01 & $31.7(13)$ & $33.8(12)$ & $42.8(14)$ & - & $3.0(11)$ & - \\
& & & & $19.6(11)$ & & $10.1(10)$ \\
O2 & $31.9(12)$ & $31.1(10)$ & $29.0(11)$ & $11.7(9)$ & $14.8(10)$ & $5.5(9)$ \\
O3 & $32.6(11)$ & $21.4(9)$ & $26.1(10)$ & $9.5(8)$ & $7.7(9)$ & $4.8(8)$ \\
C14 & $29.0(16)$ & $16.3(11)$ & $26.8(15)$ & $0.5(10)$ & $0.4(11)$ & $-0.8(10)$ \\
\hline & & & & & &
\end{tabular}

Table S12: Bond Lengths for $7 k$.

\begin{tabular}{|c|c|c|c|c|c|}
\hline Atom & Atom & Length/Å & Atom & Atom & Length/Å \\
\hline I1 & C4A & $2.106(2)$ & C11 & $\mathrm{C} 12$ & $1.395(4)$ \\
\hline 11 & C5A & $2.107(3)$ & C11' & C12' & $1.398(4)$ \\
\hline 1002 & C24A & $2.098(2)$ & $\mathrm{C} 12$ & C13 & $1.384(5)$ \\
\hline 1002 & $C 25 A$ & $2.111(3)$ & C12' & $\mathrm{C} 13$ & $1.383(5)$ \\
\hline S21 & 021 & $1.447(2)$ & $\mathrm{C} 21$ & $\mathrm{C} 22$ & $1.395(4)$ \\
\hline S21 & $\mathrm{O} 22$ & $1.438(2)$ & $\mathrm{C} 21$ & C29A & $1.397(4)$ \\
\hline S21 & 023 & $1.447(2)$ & $\mathrm{C} 22$ & $\mathrm{C} 23$ & $1.383(4)$ \\
\hline$S 21$ & C34 & $1.823(3)$ & $\mathrm{C} 23$ & $\mathrm{C} 24$ & $1.391(4)$ \\
\hline F21 & C34 & $1.317(4)$ & $\mathrm{C} 24$ & C24A & $1.390(4)$ \\
\hline F22 & C34 & $1.332(4)$ & C24A & C29A & $1.392(4)$ \\
\hline F23 & C34 & $1.330(4)$ & C25A & C28A & $1.388(4)$ \\
\hline COAA & C25A & $1.389(4)$ & $\mathrm{C} 26$ & $\mathrm{C} 27$ & $1.384(5)$ \\
\hline COAA & $\mathrm{C} 26$ & $1.390(4)$ & $\mathrm{C} 27$ & $\mathrm{C} 28$ & $1.385(4)$ \\
\hline C1 & $\mathrm{C} 2$ & $1.389(4)$ & $\mathrm{C} 28$ & C28A & $1.406(4)$ \\
\hline C1 & C9A & $1.399(4)$ & C28A & $\mathrm{C} 29$ & $1.515(4)$ \\
\hline
\end{tabular}




\begin{tabular}{llllll}
\hline C2 & C3 & $1.381(4)$ & C29 & C29A & $1.516(3)$ \\
C3 & C4 & $1.388(3)$ & C29 & C30 & $1.548(3)$ \\
C4 & C4A & $1.385(4)$ & C30 & C31 & $1.391(4)$ \\
C4A & C9A & $1.388(4)$ & C30 & C31' & $1.392(4)$ \\
C5 & C5A & $1.385(3)$ & C31 & C32 & $1.399(4)$ \\
C5 & C6 & $1.388(4)$ & C31' & C32' & $1.396(4)$ \\
C5A & C8A & $1.395(4)$ & C32 & C33 & $1.380(4)$ \\
C6 & C7 & $1.377(5)$ & C32' & C33 & $1.377(4)$ \\
C7 & C8 & $1.398(4)$ & S1 & O1 & $1.450(2)$ \\
C8 & C8A & $1.396(4)$ & S1 & O2 & $1.433(2)$ \\
C8A & C9 & $1.519(3)$ & S1 & O3 & $1.442(2)$ \\
C9 & C9A & $1.512(3)$ & S1 & C14 & $1.826(3)$ \\
C9 & C10 & $1.538(3)$ & F1 & C14 & $1.337(4)$ \\
C10 & C11 & $1.398(4)$ & F2 & C14 & $1.328(4)$ \\
C10 & C11 & $1.390(4)$ & F3 & C14 & $1.326(4)$ \\
\hline
\end{tabular}

Table S13: Bond Angles for $7 \mathbf{k}$.

\begin{tabular}{|c|c|c|c|c|c|c|c|}
\hline Atom & Atom & Atom & Angle $^{\circ}$ & Atom & Atom & Atom & Angle $^{\circ}$ \\
\hline C4A & 11 & C5A & $89.99(10)$ & $\mathrm{C} 24$ & C24A & 1002 & $116.88(18)$ \\
\hline C24A & 1002 & C25A & $91.51(11)$ & $\mathrm{C} 24$ & C24A & C29A & $124.3(2)$ \\
\hline 021 & S21 & C34 & $102.70(16)$ & C29A & C24A & 1002 & $118.78(18)$ \\
\hline 022 & S21 & 021 & $114.94(15)$ & COAA & $C 25 \mathrm{~A}$ & 1002 & $117.5(2)$ \\
\hline 022 & S21 & $\mathrm{O} 23$ & $115.05(14)$ & C28A & C25A & 1002 & $118.14(18)$ \\
\hline 022 & S21 & C34 & $104.10(15)$ & $\mathrm{C} 28 \mathrm{~A}$ & $\mathrm{C} 25 \mathrm{~A}$ & COAA & $124.3(2)$ \\
\hline 023 & S21 & 021 & $113.97(13)$ & $\mathrm{C} 27$ & $\mathrm{C} 26$ & COAA & $120.4(3)$ \\
\hline 023 & S21 & C34 & $103.98(14)$ & $\mathrm{C} 26$ & $\mathrm{C} 27$ & $\mathrm{C} 28$ & $120.7(3)$ \\
\hline C25A & COAA & $\mathrm{C} 26$ & $117.4(3)$ & $\mathrm{C} 27$ & $\mathrm{C} 28$ & C28A & $120.8(3)$ \\
\hline C2 & $\mathrm{C} 1$ & C9A & $120.5(3)$ & $\mathrm{C} 25 \mathrm{~A}$ & $\mathrm{C} 28 \mathrm{~A}$ & $\mathrm{C} 28$ & $116.2(2)$ \\
\hline C3 & $\mathrm{C} 2$ & $\mathrm{C} 1$ & $121.1(2)$ & C25A & C28A & $\mathrm{C} 29$ & $124.0(2)$ \\
\hline C2 & $\mathrm{C} 3$ & $\mathrm{C} 4$ & $119.8(2)$ & $\mathrm{C} 28$ & C28A & $\mathrm{C} 29$ & $119.6(2)$ \\
\hline C4A & $\mathrm{C} 4$ & $\mathrm{C} 3$ & $118.1(3)$ & C28A & $\mathrm{C} 29$ & C29A & $113.6(2)$ \\
\hline C4 & $\mathrm{C} 4 \mathrm{~A}$ & 11 & $118.99(19)$ & $\mathrm{C} 28 \mathrm{~A}$ & $\mathrm{C} 29$ & $\mathrm{C} 30$ & $110.3(2)$ \\
\hline C4 & $\mathrm{C} 4 \mathrm{~A}$ & C9A & $123.9(2)$ & C29A & $\mathrm{C} 29$ & C30 & $113.8(2)$ \\
\hline C9A & $\mathrm{C} 4 \mathrm{~A}$ & 11 & $117.12(18)$ & $\mathrm{C} 21$ & C29A & $\mathrm{C} 29$ & $120.3(2)$ \\
\hline C5A & C5 & $\mathrm{C} 6$ & $117.9(3)$ & C24A & C29A & $\mathrm{C} 21$ & $116.2(2)$ \\
\hline C5 & C5A & 11 & $119.5(2)$ & C24A & C29A & $\mathrm{C} 29$ & $123.5(2)$ \\
\hline C5 & C5A & C8A & $123.7(2)$ & C31 & $\mathrm{C} 30$ & $\mathrm{C} 29$ & $120.9(2)$ \\
\hline C8A & C5A & 11 & $116.82(18)$ & C31 & C30 & $\mathrm{C} 31^{\prime}$ & $118.6(2)$ \\
\hline C7 & C6 & $\mathrm{C} 5$ & $120.3(3)$ & C31' & C30 & C29 & $120.3(2)$ \\
\hline C6 & $\mathrm{C} 7$ & $\mathrm{C} 8$ & $121.1(3)$ & C30 & C31 & C32 & $120.2(3)$ \\
\hline C8A & $\mathrm{C} 8$ & $\mathrm{C} 7$ & $120.1(3)$ & C30 & C31' & C32' & $120.7(2)$ \\
\hline C5A & C8A & $\mathrm{C} 8$ & $116.9(2)$ & C33 & C32 & $\mathrm{C} 31$ & $120.6(3)$ \\
\hline C5A & C8A & C9 & $121.5(2)$ & C33 & C32' & C31' & $120.3(3)$ \\
\hline C8 & C8A & C9 & $121.6(2)$ & C32' & C33 & C32 & $119.6(2)$ \\
\hline C8A & C9 & $\mathrm{C} 10$ & $111.8(2)$ & F21 & C34 & S21 & $111.6(3)$ \\
\hline C9A & C9 & C8A & $110.44(19)$ & F21 & C34 & F22 & $107.6(3)$ \\
\hline C9A & C9 & $\mathrm{C} 10$ & $110.8(2)$ & $F 21$ & C34 & $F 23$ & $107.6(3)$ \\
\hline C1 & C9A & $\mathrm{C} 9$ & $122.1(2)$ & $\mathrm{F} 22$ & C34 & S21 & $110.7(2)$ \\
\hline C4A & C9A & $\mathrm{C} 1$ & $116.5(2)$ & F23 & C34 & S21 & $110.8(2)$ \\
\hline C4A & $\mathrm{C9A}$ & C9 & $121.3(2)$ & $\mathrm{F} 23$ & C34 & $\mathrm{F} 22$ & $108.3(3)$ \\
\hline
\end{tabular}




\begin{tabular}{llllllll}
\hline C11 & C10 & C9 & $119.4(2)$ & O1 & S1 & C14 & $101.41(15)$ \\
C11' & C10 & C9 & $121.5(2)$ & O2 & S1 & O1 & $114.70(16)$ \\
C11' & C10 & C11 & $119.1(2)$ & O2 & S1 & O3 & $115.05(14)$ \\
C12 & C11 & C10 & $120.4(3)$ & O2 & S1 & C14 & $105.05(14)$ \\
C10 & C11' & C12' & $120.2(3)$ & O3 & S1 & O1 & $114.61(15)$ \\
C13 & C12 & C11 & $120.0(3)$ & O3 & S1 & C14 & $103.79(14)$ \\
C13 & C12' & C11' & $120.2(3)$ & F1 & C14 & S1 & $110.0(2)$ \\
C12' & C13 & C12 & $120.0(3)$ & F2 & C14 & S1 & $111.4(2)$ \\
C22 & C21 & C29A & $120.7(3)$ & F2 & C14 & F1 & $107.3(3)$ \\
C23 & C22 & C21 & $121.4(3)$ & F3 & C14 & S1 & $111.8(2)$ \\
C22 & C23 & C24 & $119.5(3)$ & F3 & C14 & F1 & $108.3(3)$ \\
C24A & C24 & C23 & $117.9(2)$ & F3 & C14 & F2 & $107.8(3)$ \\
\hline
\end{tabular}

Table S14: Hydrogen Atom Coordinates $\left(\AA \times 10^{4}\right)$ and Isotropic Displacement Parameters $\left(\AA^{2} \times 10^{3}\right)$ for 7 k.

\begin{tabular}{|c|c|c|c|c|}
\hline Atom & $x$ & $y$ & $z$ & $U(\mathrm{eq})$ \\
\hline HOAA & 3533.29 & 1066.65 & 9204.9 & 28 \\
\hline H1 & 5118.69 & 1222.03 & 3058.98 & 24 \\
\hline $\mathrm{H} 2$ & 4072.94 & 277.81 & 2877.13 & 27 \\
\hline H3 & 2639.06 & 184.65 & 3714.05 & 25 \\
\hline H4 & 2168.92 & 1099.1 & 4669.28 & 20 \\
\hline H5 & 2491.12 & 3953.66 & 4377.66 & 23 \\
\hline H6 & 3199.31 & 4700.16 & 3309.99 & 31 \\
\hline H7 & 4654.43 & 4396.1 & 2608.49 & 32 \\
\hline H8 & 5450.02 & 3354.35 & 2958.06 & 27 \\
\hline H9 & 5545.72 & 2290.98 & 3745.73 & 19 \\
\hline H11 & 5393.2 & 1300.62 & 5257.22 & 25 \\
\hline H11' & 5380.33 & 3404.41 & 5284.22 & 25 \\
\hline H12 & 5945.42 & 1291.8 & 6747.13 & 31 \\
\hline H12' & 5935.14 & 3389.37 & 6774.68 & 31 \\
\hline H13 & 6221.94 & 2336.51 & 7500 & 35 \\
\hline H21 & 5650.46 & 3952.21 & 11064.47 & 25 \\
\hline $\mathrm{H} 22$ & 4621.95 & 4911.62 & 10919 & 30 \\
\hline $\mathrm{H} 23$ & 3349.22 & 4921 & 9878.52 & 28 \\
\hline $\mathrm{H} 24$ & 3107.68 & 3950.51 & 8938.12 & 21 \\
\hline H26 & 4047.79 & 272.89 & 10312.04 & 36 \\
\hline $\mathrm{H} 27$ & 5216.78 & 598.69 & 11369.02 & 36 \\
\hline H28 & 5881.94 & 1709.63 & 11355.99 & 28 \\
\hline $\mathrm{H} 29$ & 6045.58 & 2807 & 10761.17 & 20 \\
\hline H31 & 6488.46 & 3730.27 & 9093.89 & 26 \\
\hline H31' & 6792.92 & 1675.21 & 9581.1 & 26 \\
\hline H32 & 7810.55 & 3677.86 & 8090.94 & 31 \\
\hline H32' & 8122.71 & 1634.81 & 8589.74 & 28 \\
\hline H33 & 8631.6 & 2635.01 & 7845.45 & 27 \\
\hline
\end{tabular}




\section{Literature}

[1] Wilfred L. F. Armarego, Christina Li Lin Chai, Purification of Laboratory Chemicals, Elsevier, 2009.

[2] E. Krasnokutskaya, N. Semenischeva, V. Filimonov, P. Knochel, Synthesis 2007, 2007, 81.

[3] R. Beckert, E. Fanghänel, W. D. Habicher, H.-J. Knölker, P. Metz, K. Schwetlick, Organikum. Organisch-chemisches Grundpraktikum, Wiley-VCH Verlag, Weinheim, 2015.

[4] R. Imbos, A. J. Minnaard, B. L. Feringa, J. Am. Chem. Soc. 2002, 124, 184.

[5] R. Quach, D. P. Furkert, M. A. Brimble, Tetrahedron Lett. 2013, 54, 5865.

[6] S. Wang, L. Liu, X. Guo, G. Li, X. Wang, H. Dong, Y. Li, W. Zhao, RSC Adv. 2019, 9, 13878.

[7] T. Fukuyama, T. Bando, I. Ryu, Synthesis 2018, 50, 3015.

[8] J. C. Gonzalez-Gomez, N. P. Ramirez, T. Lana-Villarreal, P. Bonete, Org. Biomol. Chem. 2017, 15, 9680.

[9] M. Barbasiewicz, K. Błocki, M. Malińska, R. Pawłowski, Dalton Trans. 2013, 42, 355.

[10] E. Coya, N. Sotomayor, E. Lete, Adv. Synth. Catal. 2015, 357, 3206.

[11] M. Sickert, H. Weinstabl, B. Peters, X. Hou, M. Lautens, Angew. Chem. Int. Ed. 2014, 53, 5147.

[12] a) T.-S. Mei, R. Giri, N. Maugel, J.-Q. Yu, Angew. Chem. Int. Ed. 2008, 47, 5215; b) Xing Chen, Marc Poirier, Yee-Shing Wong, Guang-Zhong Wu, EP0977739, 2003.

[13] H.-Y. Zhao, X.-Y. Yang, H. Lei, M. Xin, S.-Q. Zhang, Synth. Commun. 2019, 49, 1406.

[14] K. S. Yang, G. Budin, C. Tassa, O. Kister, R. Weissleder, Angew. Chem. Int. Ed. 2013, 52, 10593.

[15] F. Gohier, J. Mortier, J. Org. Chem. 2003, 68, 2030.

[16] T.-C. Chang, S. J. Yu, Synth. Commun. 2014, 45, 651.

[17] A. J. Blake, A. Novak, M. Davies, R. I. Robinson, S. Woodward, Synth. Commun. 2009, 39, 1065.

[18] R. Bedford, T. Gallagher, D. Pye, W. Savage, Synthesis 2015, 47, 1761.

[19] A. Boelke, E. Lork, B. J. Nachtsheim, Chem. Eur. J. 2018, 24, 18653.

[20] J. Cvengroš, D. Stolz, A. Togni, Synthesis 2009, 2009, 2818.

[21] S. Bhattacharjee, A. Guin, R. N. Gaykar, A. T. Biju, Org. Lett. 2019, 21, 4383.

[22] D. Zhu, M. Li, Z. Wu, Y. Du, B. Luo, P. Huang, S. Wen, Eur. J. Org. Chem. 2019, 2019, 4566.

[23] C. Adamo, V. Barone, J. Chem. Phys 1999, 110, 6158.

[24] a) S. Grimme, J. Antony, S. Ehrlich, H. Krieg, J. Chem. Phys. 2010, 132, 154104; b) S. Grimme, S. Ehrlich, L. Goerigk, J. Comput. Chem. 2011, 32, 1456.

[25] F. Weigend, R. Ahlrichs, Phys. Chem. Chem. Phys. 2005, 7, 3297.

[26] F. Weigend, Phys. Chem. Chem. Phys. 2006, 8, 1057.

[27] S. Kozuch, D. Gruzman, J. M. L. Martin, J. Phys. Chem. C 2010, 114, 20801.

[28] L. Goerigk, A. Hansen, C. Bauer, S. Ehrlich, A. Najibi, S. Grimme, Phys. Chem. Chem. Phys. 2017, 19, 32184.

[29] A. Hellweg, C. Hättig, S. Höfener, W. Klopper, Theor. Chem. Acc. 2007, 117, 587. 


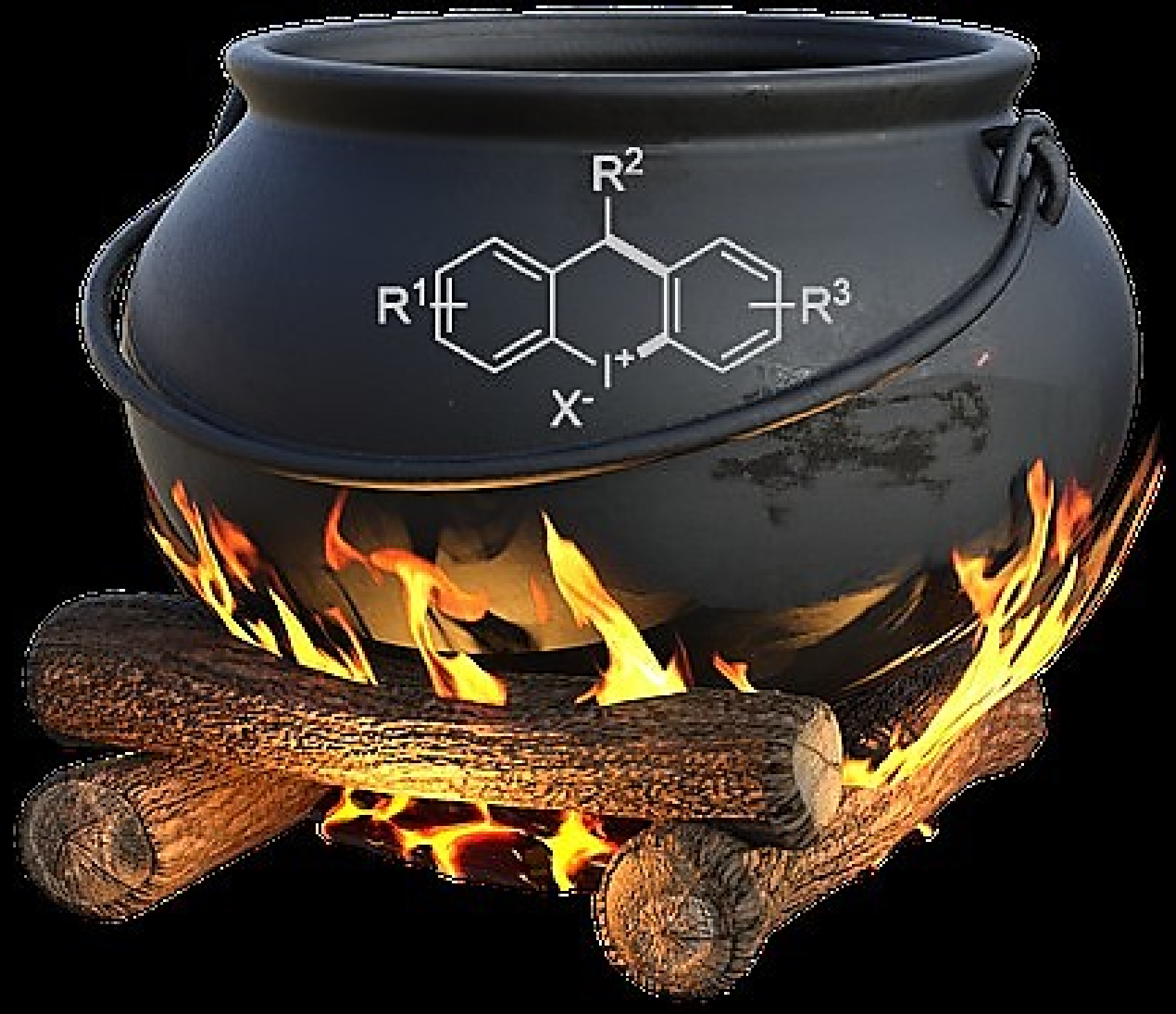

graphical abstract.jpg (50.04 KiB)

view on ChemRxiv • download file 


\section{One-Pot Synthesis and Conformational Analysis of 6- Membered Cyclic Iodonium Salts}

Lucien D. Caspers ${ }^{a}$, Julian Spils ${ }^{a}$, Mattis Damrath ${ }^{a}$, Enno Lork ${ }^{b}$ and Boris J. Nachtsheim ${ }^{\text {a* }}$

aAddress: Institute for Organic and Analytical Chemistry, University of Bremen, 28359 Bremen, Germany, phone: +49 421 218-63113

bAddress: Institute for Inorganic Chemistry and Crystallography, University of Bremen, 28359 Bremen, Germany, phone: +49 421 218-63155

Prof. Dr. Boris J. Nachtsheim, nachtsheim@uni-bremen.de

\section{Abstract}

Two one-pot procedures for the construction of carbon-bridged diaryliodonium triflates and tetrafluoroborates are described. Strong Brønsted acids enable the effective Friedel-Crafts alkylation with diversely substituted o-iodobenzylalcohol derivatives, providing diphenylmethane scaffolds which are subsequently oxidized and cyclized to the corresponding dibenzo[b,e]iodininium salts. Based on NMR-investigations and DFT-calculations we could verify a so far undescribed existence of two stable isomers in cyclic iodonium salts substituted with aliphatic side chains in the carbon bridge. 


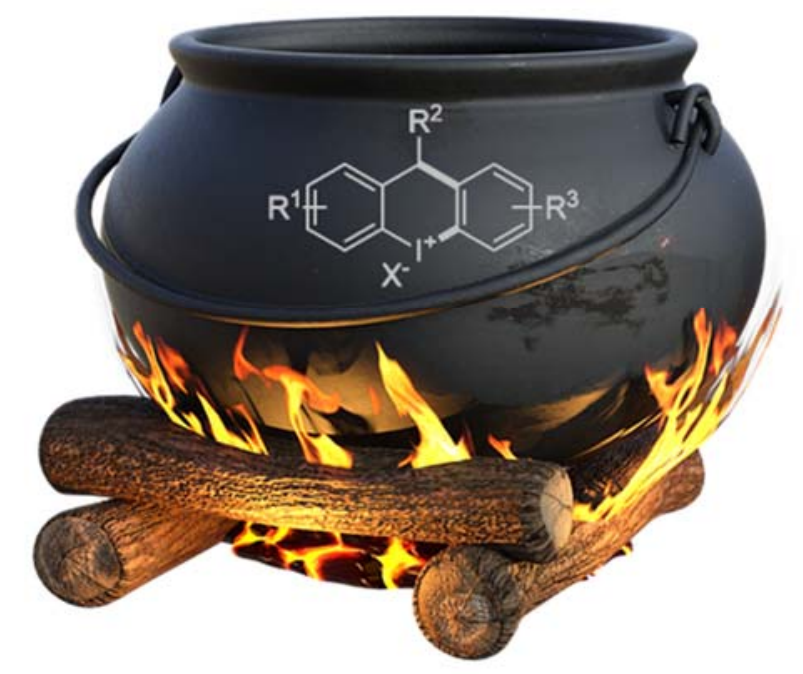

\section{Keywords}

hypervalent iodine - iodonium salts - one-pot synthesis • Friedel-crafts synthesis • I-heterocycles

\section{Introduction}

The last decades have witnessed extensive developments in the chemistry of hypervalent iodine compounds (HVIs). ${ }^{[1]}$ Even in the delicate field of total synthesis ${ }^{[2]}$ or in organo-[3] and photocatalysis, ${ }^{[4]} \mathrm{HVIs}$ have found widespread applications. An important subclass of HVIs are diaryl- $\lambda^{3}$-iodanes or diaryliodonium salts (DIS, 1, Figure 1). ${ }^{[5]}$ Bearing iodobenzene as potent leaving group (hypernucleofug) ${ }^{[6]}$ they have become frequently utilized reagents in a plethora of $\mathrm{C}\left(\mathrm{sp}^{\mathrm{n}}\right)-\mathrm{H}$ and $\mathrm{Het}-\mathrm{H}$ arylation reactions. ${ }^{[7]}$ Despite their outstanding performance as electrophilic group transfer reagents in transition metal-mediated and metal-free arylations their time-consuming multi-step synthesis often impedes their application. ${ }^{[8]}$ Albeit various $\lambda^{3}$-iodane mediated reactions have been performed in a catalytic manner and diverse atomeconomic conversions have been described for different types of (recyclable) HVIs, most DIS are utilized generating equimolar amounts of waste. 


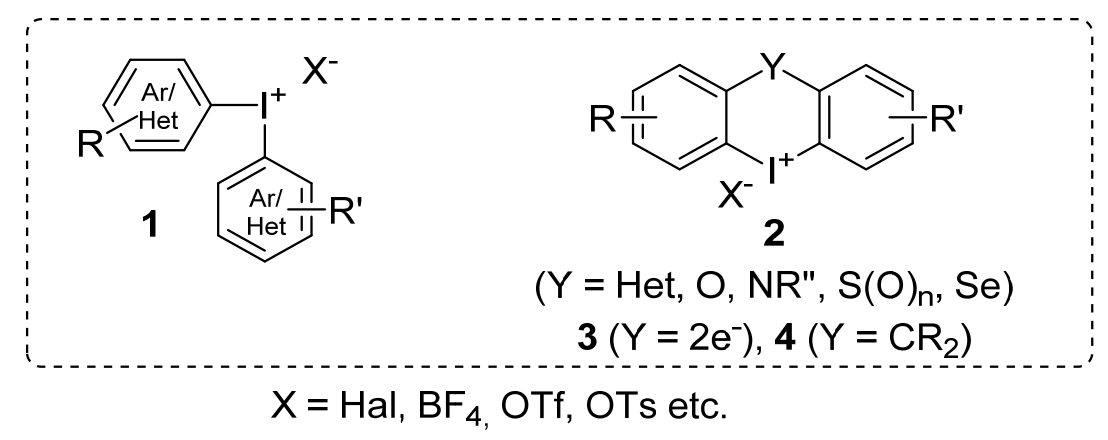

Figure 1: Schematic presentation of DIS 1 (left) and CDIS 2 - 4 (right).

A subclass of DIS are cyclic diaryliodonium salts (CDIS) where the aryl moieties are directly connected or bridged with carbon- or heteroatoms $\left(2-4\right.$, Figure 1) ${ }^{[9]}$ While comprising a comparable reactivity towards carbon and heteroatom nucleophiles as their acyclic counterparts, CDIS are promising candidates for the effective synthesis of doubly functionalized diaryl-based systems. The aryl-moieties are chemically bound and are, after an initial ring-opening reaction, readily available for a second inter- or intramolecular coupling event. Various transformations have been described with these iodacycles providing numerous highly functionalized biaryls and polycyclic (hetero)arenes (Scheme 1). Here, tricyclic dibenzo[b,d]iodol-5-ium salts $\mathbf{3}$ were widely applied. They have been utilized in enantioselective ring opening reactions to give $o, 0$ 'difunctionalized biaryls (Scheme 1, a) ${ }^{[10]}$ and in effective double functionalizations for the efficient synthesis of carbazoles, fluorenes, dibenzothio- and dibenzoselenophenes (b), ${ }^{[11,12,13]}$ giving a fast access to a wide range of pharmacophores and functional materials. 6-Membered dibenzo[b,e]iodininium salts 4 were commonly utilized as well. Effective ring-opening reactions with high enantioselectivities (c), ${ }^{[14]}$ and the synthesis of (oxidized) thioxanthenes (d), ${ }^{[12]}$ acridines $(\mathbf{e})^{[13]}$ and xanthen(on)es $(\mathbf{f})^{[15]}$ have been described, emphasizing their wide range of applications. 


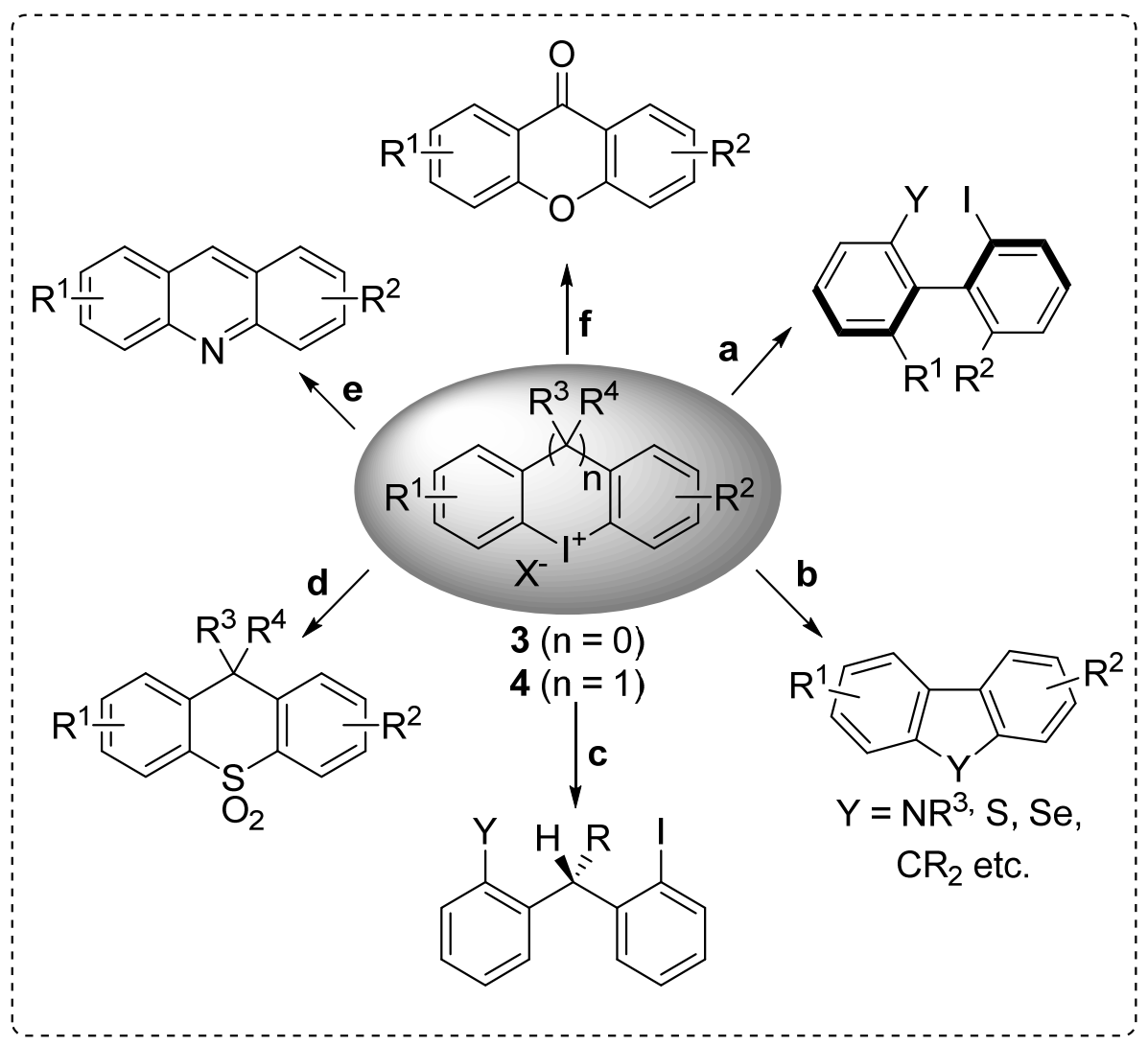

Scheme 1: Synthetic applications of cyclic diaryliodonium salts.

Compared to their acyclic counterparts, CDIS are much less frequently utilized in organic synthesis. Apart from their specialized need as substrates for the synthesis of heteroatom- or carbon-bridged $0,0^{\prime}$-disubstituted biaryl systems, one reason for this issue may be the availability of efficient protocols for their synthesis. The synthesis of iodolium salts $\mathbf{3}$ and their precursors is commonly achieved via cross-coupling reactions of $o$-haloanilines followed by a Sandmeyer-type iodination (Scheme 2, A). ${ }^{13}$ ] The preparation of homologues iodininium salts 4 is less standardized: A selective mono-metalation of $o$-diiodobenzenes and 1,2-addition to aromatic aldehydes with a subsequent dehydroxylation enables the synthesis of various diphenylmethanes (Scheme 2, B1). ${ }^{[13]}$ Different $p$-toluidine derivatives have been $o$-alkylated in order to obtain diversely aminated 2,2-diphenylpropanes which were transformed into precursors for CDIS via Sandmeyer iodination (B2). ${ }^{[12] ~ U n s u b s t i t u t e d ~}$ diphenylmethanes can be modified with electron-withdrawing groups, thus enabling a selective electrophilic iodination at the methylene o-position (B3). ${ }^{[16]}$ 
A) Common pathway for the synthesis of o-iodobiphenyls

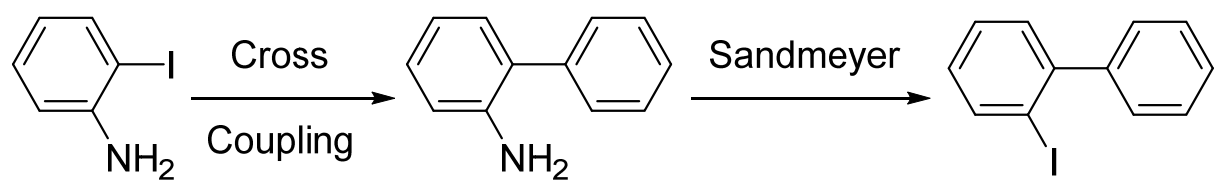

B) Conventional syntesis of dibenzo[b,e]iodinium precursors<smiles>[R]C(=O)c1ccc(I)c(C(O)c2cccc(Cc3ccccc3)c2)c1</smiles><smiles>Cc1ccc(N)cc1</smiles><smiles>N#Cc1ccc(Cc2ccc([N+](=O)[O-])cc2)c(I)c1</smiles>

C) Our approach: One-pot Friedel-Crafts/oxidation sequence

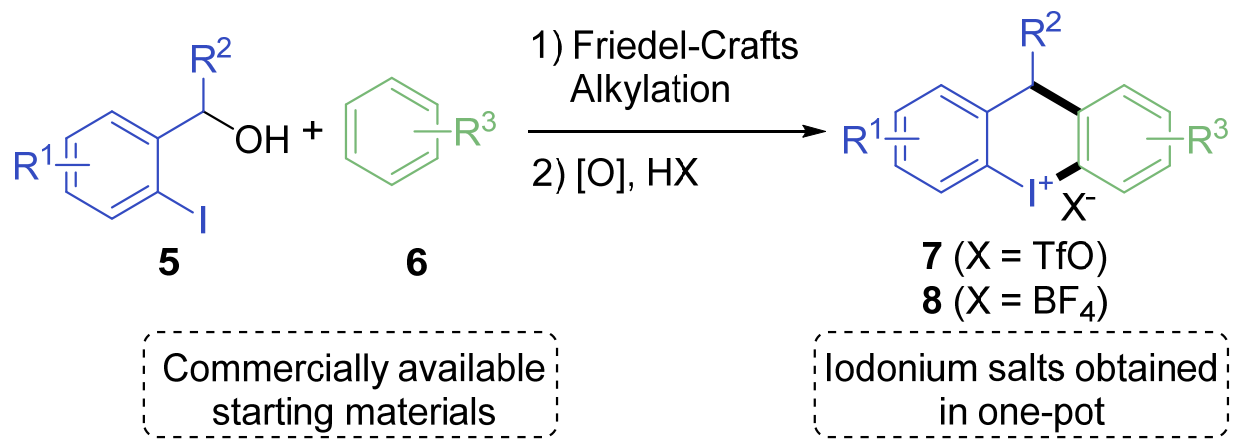

Scheme 2: Common pathways for the precursor synthesis of cyclic diaryliodonium salts.

All these routes need at least two independent steps to obtain the $o$-iodinated diphenylcompounds which then need to be further oxidized to the desired CDIS $\mathbf{3}$ or $\mathbf{4}$. Herein, we describe the effective synthesis of dibenzo[b,e]iodininium salts 4 in a one-step Friedel-Crafts/oxidation sequence to give iodininium triflates and tetrafluoroborates 7 and 8 (Scheme 2, C). 


\section{Results and Discussion}

For the initial Friedel-Crafts alkylation with o-iodobenzyl alcohols $\mathbf{5}$ different procedures in dipolar aprotic solvents were known under Brønsted or Lewis-acidic conditions. ${ }^{[17]}$ We decided to utilize a strong but weakly coordinating Brønsted acid that promotes the coupling step to the diphenylmethane 9 (Table 1) and the concluding oxidation to the final dibenzo[b,e]iodininium salt 4 to serve as the activator in both steps and as a the final counter anion in the CDIS. In preliminary optimizations with trifluoromethanesulfonic acid (TfOH) and mCPBA as oxidant (see SI, Table S1) the desired one-pot reaction was effectively performed. However, the utilization of $m C P B A$ gave undesired side products that complicated the work-up of the reaction. Therefore, we decided to conduct a second optimization with Oxone ${ }^{\circledR}$ as oxidant, that enabled an easier, extraction-based work-up procedure.

From literature and our preliminary test reactions we recognized that for a successful oxidation of iodoarenes with Oxone ${ }^{\circledR}$, combinations of $\mathrm{TfOH}$ and $\mathrm{H}_{2} \mathrm{SO}_{4}$ are best in terms of reactions times and yields (SI, Table 2) and therefore focused on this combination. ${ }^{[18]}$ We started our investigation with o-iodobenzyl alcohol 5a $(\mathrm{R}=\mathrm{H}$, Table 1) as the model substrate. Coupling with $\mathrm{TfOH}$ and 3 eq. of benzene (6a) in DCM and subsequent oxidation with $\mathrm{Oxone}^{\circledR}$ and $\mathrm{H}_{2} \mathrm{SO}_{4}$ after addition of $\mathrm{MeCN}$ yielded the desired iodonium salt $\mathbf{7 a}$ in $36 \%$ (entry 1 ). While the yield was still moderate, the product could be easily purified by extraction and washing. Utilizing 5 eq. of $6 \mathbf{a}$ enhanced the yield to $77 \%$ (entry 2 ) while the use 10 eq. improved the product purity due to a lowered side product profile in the coupling step (entry 3). A dropwise addition of $\mathbf{5 a}$ to the acidic solution led to a more selective generation of $9 \mathbf{a}$ and could increase the yield to $89 \%$ (entry 4). Performing the first step in a $1: 1$ mixture of benzene and DCM did not show a significant improvement (entry 5). Further experiments revealed, that $\mathrm{MeCN}$ was necessary in the second step to ensure a clean conversion of the intermediate (entry 6 ) while the generation of 9 a could not be achieved in pure MeCN (entry 7). Addition of low amounts of 1,1,1,3,3,3-hexafluoroisopropanol (HFIP) in the second reaction step slightly improved the reaction time (entry 8).

When we then started to investigate our substrate scope, we observed a significant dropdown in yield with brominated o-iodobenzylic alcohol 5b (entry 9). Addition of HFIP in the second step could increase the oxidation rate and product $\mathbf{7 b}$ was obtained in 
$85 \%$ (entry 10). With $\mathrm{NO}_{2}$-substituted alcohol $\mathbf{5 c}$ addition of HFIP was not sufficient to completely convert the intermediate 9c (entry 11). Possibly due to diminished stability of the oxidant under these strongly acidic conditions, the addition of further Oxone ${ }^{\circledR}$ and $\mathrm{H}_{2} \mathrm{SO}_{4}$ after an initial oxidation period was necessary which finally increased the yield of $7 \mathrm{c}$ to $84 \%$ (entry 12 ).

Table 1: Optimization of the One-Pot Procedure with Oxone ${ }^{\circledR}$ as Oxidant.

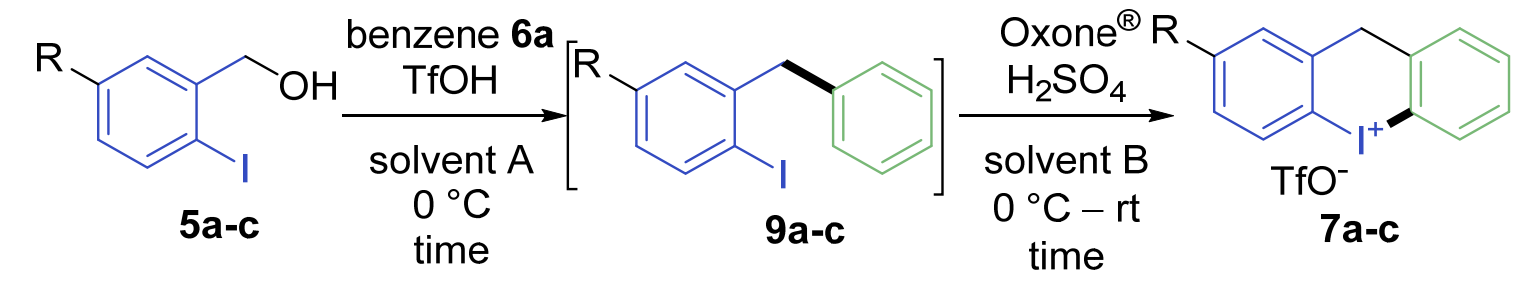

\begin{tabular}{|c|c|c|c|c|c|c|}
\hline Entry ${ }^{[a]}$ & $\mathrm{R}$ & $\begin{array}{l}\text { Benzene } \\
\text { (eq.) }\end{array}$ & $\begin{array}{c}\text { Solvent } \\
\text { A }\end{array}$ & $\begin{array}{c}\text { Solvent } \\
\text { B }\end{array}$ & $\begin{array}{c}\mathrm{t}_{1} / \mathrm{t}_{2} \\
{[\mathrm{~min}] /[\mathrm{h}]}\end{array}$ & $\begin{array}{c}\text { Yield of } 7^{[\mathrm{b}]} \\
{[\%]}\end{array}$ \\
\hline 1 & $H(5 a)$ & 3 & DCM & $\mathrm{MeCN}$ & $5 / 1.5$ & 36 \\
\hline 2 & $\mathrm{H}$ & 5 & DCM & $\mathrm{MeCN}$ & $5 / 1.5$ & 77 \\
\hline 3 & $\mathrm{H}$ & 10 & DCM & $\mathrm{MeCN}$ & $5 / 1.5$ & $71^{[\mathrm{c}]}$ \\
\hline $4^{[\mathrm{d}]}$ & $\mathbf{H}$ & 10 & DCM & $\mathrm{MeCN}$ & $5 / 2$ & 89 \\
\hline $5^{[\mathrm{d}]}$ & $\mathrm{H}$ & 28 & $\mathrm{DCM}$ & $\mathrm{MeCN}$ & $5 / 2$ & 90 \\
\hline $6^{[\mathrm{d}]}$ & $\mathrm{H}$ & 10 & $\mathrm{DCM}$ & DCM & $5 / 24$ & $<10$ \\
\hline $7^{[\mathrm{d}]}$ & $\mathrm{H}$ & 10 & $\mathrm{MeCN}$ & $\mathrm{MeCN}$ & 360 / --- & 0 \\
\hline $8^{[\mathrm{d}]}$ & $\mathrm{H}$ & 10 & $\mathrm{DCM}$ & $\operatorname{MeCN}^{[e]}$ & $5 / 1$ & 89 \\
\hline 9 & $\mathrm{Br}(5 b)$ & 10 & DCM & $\mathrm{MeCN}$ & $120 / 16$ & 57 \\
\hline 10 & $\mathrm{Br}$ & 10 & DCM & $\operatorname{MeCN}^{[e]}$ & $120 / 16$ & 85 \\
\hline 11 & $\mathrm{NO}_{2}(5 \mathrm{c})$ & 10 & DCM & $\mathrm{MeCN}^{[\mathrm{e}]}$ & $300 / 16$ & $40-50$ \\
\hline $12^{[f]}$ & $\mathrm{NO}_{2}$ & 10 & DCM & $\operatorname{MeCN}[\mathrm{e}]$ & $300 / 40$ & 84 \\
\hline
\end{tabular}

[a] Reaction conditions: Step 1: $\mathbf{5 a}(0.2 \mathrm{mmol})$, benzene, $\mathrm{TfOH}$ (5 eq.), solvent $\mathrm{A}(1 \mathrm{~mL}), 5 \mathrm{~min}$, $0{ }^{\circ} \mathrm{C}$; Step 2: solvent $\mathrm{B}(1 \mathrm{~mL}), \mathrm{H}_{2} \mathrm{SO}_{4}$ (5 eq.), Oxone ${ }^{\circledR}$ (1 eq.), tightly closed screw cap vial, $0{ }^{\circ} \mathrm{C}-$ rt. [b] Isolated yield after purification, purity $>97 \%$ [c] Fluctuation in yield comes from washing process. 10 eq. of arene were kept as purity improved from $>95 \%$ to $>98 \%$. [d] Dropwise addition of $\mathbf{5 a}$ to $\mathbf{6 a}$ in DCM and TfOH. [e] HFIP $(0.2 \mathrm{~mL})$ was added. [f] Addition of Oxone ${ }^{\circledR}$ (1 eq.) and $\mathrm{H}_{2} \mathrm{SO}_{4}$ (5 eq.) after $16 \mathrm{~h}$ of step 2. 
With optimized conditions for different benzylic alcohols $\mathbf{5}$ in hand, we started to further investigate the scope and scalability of the reaction (Scheme 3). Performing the reaction on a $5 \mathrm{mmol}$ scale, gave $7 \mathrm{a}$ in slightly improved yield of $93 \%$. We then investigated other leaving groups than $\mathrm{Y}=\mathrm{OH}$. When utilizing o-iodobenzyl acetate 10,7 a was obtained in $87 \%$ yield, whereas with methyl ether 11 or ethyl carbonate 12 iodonium salt $7 \mathrm{a}$ was isolated in $79 \%$ and $89 \%$ yield. With chloride 13 a complex miture was obtained during the oxidation step which drastically reduced isolated yield of $7 \mathrm{a}$ to less than $10 \%$.

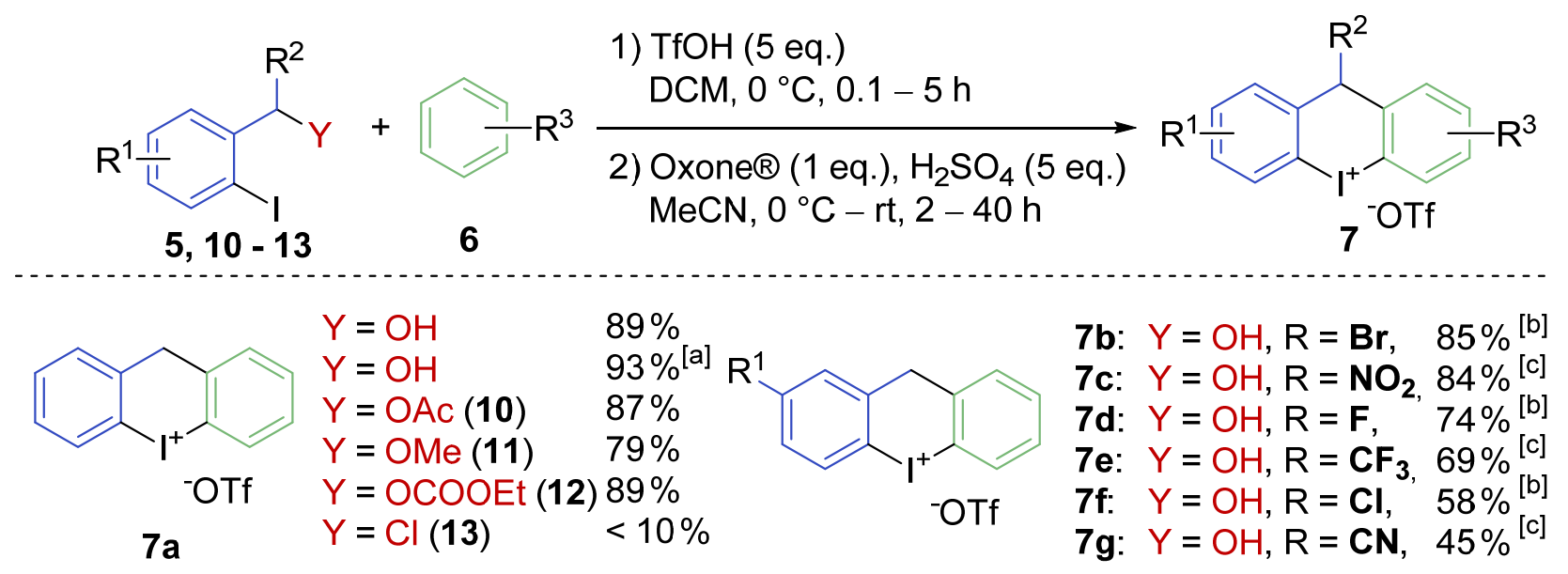

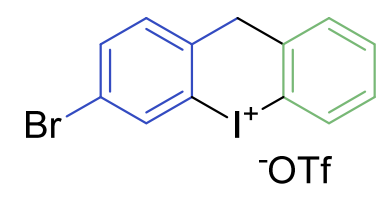

7h: $\mathrm{Y}=\mathrm{OH}, 81 \%[\mathrm{~b}]$

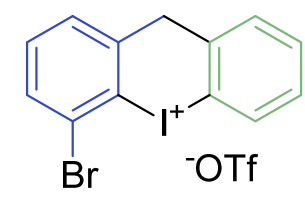

7i: $Y=O H, 55 \%[b]$

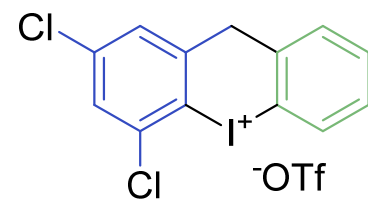

7j: $Y=O H, 42 \%{ }^{[b]}$

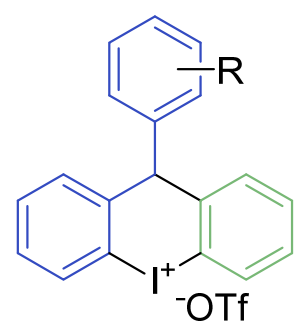

$\mathbf{7 k}: \mathrm{Y}=\mathrm{OH}, \mathrm{R}=\mathbf{H}, 79 \%$

7I: $Y=O H, R=3,5-\left(C_{3}\right), 73 \%$

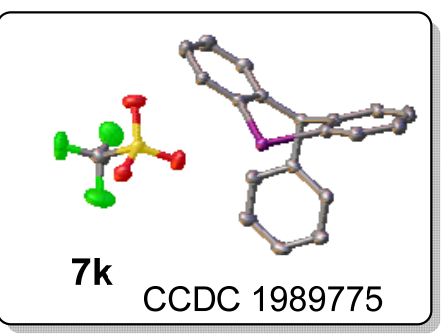

98775

Scheme 3: Substrate scope using the optimized conditions of Table 1: Step 1: 5 (0.2 mmol), 6 (10 eq.), TfOH (5 eq.), DCM (1 mL), 5 min, $0{ }^{\circ} \mathrm{C}$; Step 2: $\mathrm{MeCN}(1 \mathrm{~mL}), \mathrm{H}_{2} \mathrm{SO}_{4}$ (5 eq.), Oxone $^{\circledR}$ (1 eq.), tightly closed screw cap vial, $0{ }^{\circ} \mathrm{C}-$ rt. [a] Reaction was performed on a $5.0 \mathrm{mmol}$ scale. [b] HFIP $(0.2 \mathrm{~mL})$ was used in step 2. [c] $0.2 \mathrm{~mL}$ HFIP were used in step 2. After $16 \mathrm{~h}$ additional Oxone ${ }^{\circledR}$ (1 eq.) and $\mathrm{H}_{2} \mathrm{SO}_{4}$ (5 eq.) were added. [d] Only 5 eq. of 6 were used. 
As already discussed in Table $1,5-\mathrm{Br}$ and $5-\mathrm{NO}_{2}$ derivatives were viable as well, although under slightly different reaction conditions. Under these conditions F- and $\mathrm{CF}_{3}$-substituted derivatives $\mathbf{7 d}$ and $7 \mathrm{e}$ could be obtained in up to $74 \%$. Cl-substitution led to a diminished yield of $58 \%$ for $\mathbf{7 f}$. $\mathrm{CN}$-substituted iodonium salt $\mathbf{7 g}$ was generated in $45 \%$, albeit the rate for both reaction steps was significantly reduced. A variation of the substitution pattern was viable as 4 - and $3-B r-s u b s t i t u t e d$ derivatives $\mathbf{7 h}$ and $\mathbf{7 i}$ were isolated in $81 \%$ and $55 \%$ respectively, indicating that steric effects may be relevant during the oxidation step. Thus, the lowered yield for the $(\mathrm{Cl})_{2}$-substituted salt 7j (42\%) in comparison to $7 \mathbf{f}$ might be due to electronic or steric effects. The utilization of secondary aliphatic benzyl alcohols was difficult due to significant styrene formation during the Friedel-Crafts step (see SI, Figure S1) while secondary diphenylmethanols were suitable. Here, the Ph-substituted salt $\mathbf{7 k}$ could be obtained in $79 \%$ yield. Even substrates with electron withdrawing substituents such as bis-( $\left.\mathrm{CF}_{3}\right)-$ and $\mathrm{Cl}, \mathrm{F}-$ disubstituted diphenylmethanols were viable for a selective cyclization and yielded $7 \mathbf{I}$ and $7 \mathrm{~m}$ in up to $73 \%$ yield.

Reaction of $\mathbf{5 a}$ with $p$-xylene successfully lead to the formation of $\mathbf{7 n}$ in $93 \%$ yield. However, arenes with an unsymmetrical substitution pattern or more electron donating groups were problematic due to inseparable isomer formation and decomposition during the oxidation step. Substrates that could not successfully be synthesized are found in the SI (Figure S1).

While the selectivities in Friedel-Crafts alkylations and the decomposition of electron rich substrates were intrinsic problems of our methodology, we tried to reoptimize our one-pot procedure to enable the transformation of secondary, aliphatic benzyl alcohols. In preexaminations we found, that 1-(2-iodophenyl)ethan-1-ol (5n, Table 2) reacts in DCM with toluene in excellent para-selectivity under mild conditions $\left(0^{\circ} \mathrm{C}\right)$ with 1 eq. of $\mathrm{HBF}_{4} \cdot \mathrm{OEt}_{2}$. When adding more acid after the addition of $m \mathrm{CPBA}$ as oxidant, the desired alkyl substituted iodonium salt 8 a could be obtained in $67 \%$ (Table 2, entry 1). TLC-MS and GC-MS investigations indicated the formation of side products during the oxidation step. Thus, a reduction of oxidant lead to a significant increase in yield ( $83 \%$, entry 2$)$. However, the reaction mixtures still contained a significant amount of dark-coloured impurities which made an unwanted filtration necessary during the work-up. Hence, we tried to modify our oxidation conditions by addition of MeCN. First, 
this resulted in a reduction in yield (entry 3). The addition of more $\mathrm{HBF}_{4}$ during the oxidation step enabled at least a moderate yield of $70 \%$ (entry 4). Reduction of the amount of oxidant was also not successful (entry 5 and SI, Table S5). We then revaluated the acid stoichiometry and recognized that addition of 3 eq. $\mathrm{HBF}_{4}$ at the beginning of the reaction was beneficial for its total outcome, yielding $81 \%$ of $\mathbf{8 a}$ (entry 6). We now were satisfied with the reaction performance and stopped our optimization at this point to investigate a preliminary substrate scope. Here we decided to use DCM as sole solvent due to its higher efficiency in terms of applied amount of $\mathrm{HBF}_{4}$.

Table 2: Re-optimization with 1-(2-iodophenyl)ethan-1-ol $(\mathbf{5 n})$ for the synthesis of iodonium salt 8a.
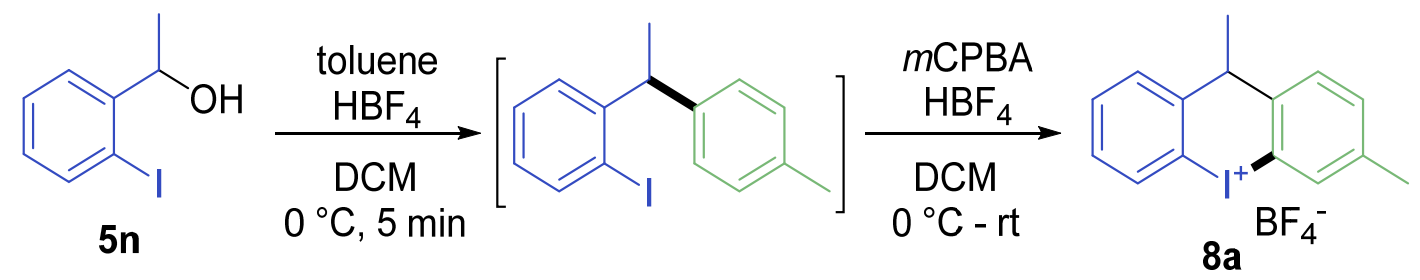

\begin{tabular}{cccccc}
\hline Entry ${ }^{[a]}$ & $\begin{array}{c}\text { PhMe } \\
(\text { eq. })\end{array}$ & $\begin{array}{c}\left.\mathrm{HBF}_{4} \text { (eq. }\right) \\
1^{\text {st }} / 2^{\text {nd }} \text { step }\end{array}$ & $\begin{array}{c}\text { mCPBA } \\
(\text { eq. })\end{array}$ & $\begin{array}{c}\text { Solvent } \\
2^{\text {nd }} \text { step }\end{array}$ & $\begin{array}{c}\text { Yield of 8a } \\
{[\%]}\end{array}$ \\
\hline 1 & 5 & $1 / 2$ & 4 & DCM & 67 \\
2 & 5 & $1 / 2$ & 2 & DCM & 83 \\
3 & 5 & $1 / 2$ & $2^{[\mathrm{b}]}$ & MeCN & 53 \\
4 & 5 & $1 / 5$ & 2 & MeCN & 70 \\
5 & 5 & $1 / 5$ & 1 & MeCN & 58 \\
6 & 5 & $3 / 2$ & 2 & MeCN & 81 \\
\hline
\end{tabular}

[a] Standard conditions: $5 \mathrm{n}(0.2 \mathrm{mmol})$ in DCM $(1 \mathrm{~mL})$ with toluene (5 eq.). Addition of $\mathrm{HBF}_{4} \cdot \mathrm{OEt}_{2}$ at $0{ }^{\circ} \mathrm{C}$, stirring till completion, addition of DCM $(1 \mathrm{~mL}), m C P B A$, stirring for $10 \mathrm{~min}$, addition of $\mathrm{HBF}_{4} \cdot \mathrm{OEt}_{2}$, stirring till completion at rt. [b] Isolated yield after purification, purity > $97 \%$. [c] $m$ CPBA (2 eq.) was added, as the intermediate was not completely converted in the $2^{\text {nd }}$ step.

When the reaction of $5 \mathrm{n}$ was performed with toluene on a $5 \mathrm{mmol}$ scale under the optimized conditions, pure 8a was obtained in 35\% (Scheme 4). When exchanging the Me-substituent to Et- or iPr-moieties the corresponding products $\mathbf{8 b}$ and $\mathbf{8 c}$ were 
isolated in yields of up to $59 \%$. The reduced yields may be attributed to a more stabilized, sterically demanding benzylic cation and a higher lability to oxidants due to the increased electron density at the benzylic position. While diphenylmethanols are well tolerated under the previously optimized conditions, the Ph-derivative $\mathbf{8 d}$ could only be obtained in $37 \%$. A reduction of the electron density of the secondary alcohol 5 was detrimental for the reaction outcome and substrate $8 \mathrm{e}$ was obtained in only $22 \%$. Due to the increased steric demand of secondary benzyl cations, the coupling with o-xylene was moderately selective and yielded $56 \%$ of $\mathbf{8 f}$. The same effect was disadvantageous for the reaction with $p$-xylene giving $\mathbf{8 g}$ in $20 \%$. With benzene as coupling partner the Friedel-Crafts step could not be performed. Also, with tertiary alcohols no product was obtained due to major side reactions during the initial FriedelCrafts-step (see SI, Figure 1).

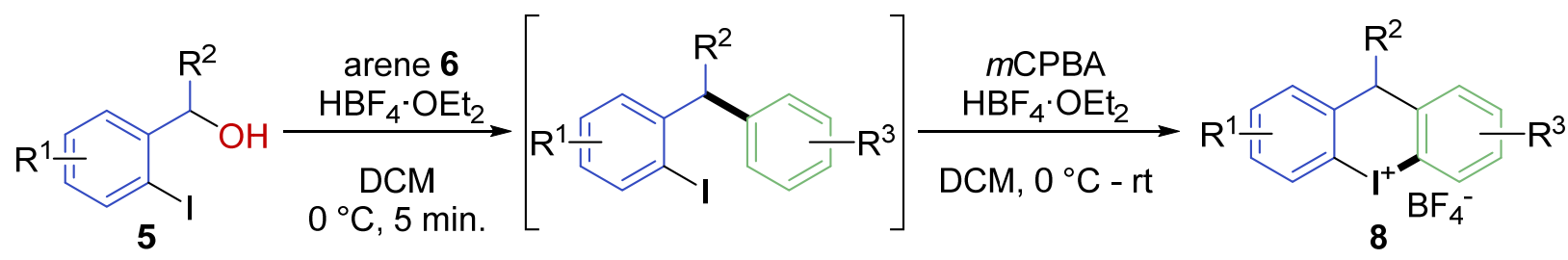

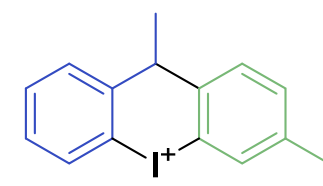

$\mathrm{BF}_{4}^{-}$

8a: $83 \%$ $35 \%{ }^{[a]}$

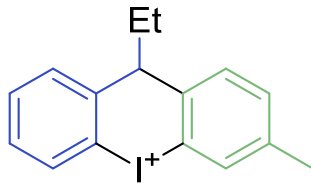

$\mathrm{BF}_{4}^{-}$

8b: $59 \%$

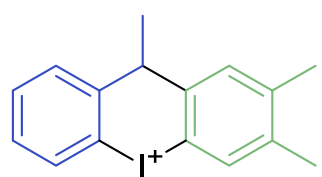

$\mathrm{BF}_{4}^{-}$<smiles>Cc1ccc2c(c1)[I-]c1ccccc1C2C(C)C</smiles>

$\mathrm{BF}_{4}^{-}$<smiles>Cc1ccc2c(c1)[I+]c1ccccc1C2c1ccccc1</smiles>

$\mathrm{BF}_{4}^{-}$ 8d: $37 \%$<smiles>Cc1ccc2c(c1)[I+]c1ccc(Br)cc1C2C</smiles>

$\mathrm{BF}_{4}^{-}$
8 e: $22 \%$ 8c: $44 \%$<smiles>Cc1cccc2c1C(C)c1ccccc1[I+]2</smiles>

$\mathrm{BF}_{4}^{-}$

8g: $20 \%$

Scheme 4: Substrate scope for the optimized conditions of Table 2: Step 1: $5(0.2 \mathrm{mmol}), 6$ (5 eq.), $\mathrm{HBF}_{4} \cdot \mathrm{OEt}_{2}$ (1 eq.), $\mathrm{DCM}(1 \mathrm{~mL}), 5 \mathrm{~min}, 0{ }^{\circ} \mathrm{C}$; Step 2: $\mathrm{DCM}(1 \mathrm{~mL}), \mathrm{HBF}_{4} \cdot \mathrm{OEt}_{2}$ (2 eq.), $m$ CPBA (2 eq.), tightly closed screw cap vial, $0^{\circ} \mathrm{C}-$ rt. [a] Reaction was performed on a $5.0 \mathrm{mmol}$ scale.

During our method development we observed that the ${ }^{1} \mathrm{H}-\mathrm{NMR}$-spectra of the newly obtained iodonium salts showed interesting exchange effects (Figure 2). Compounds $\mathbf{8} \mathbf{a}$ and $\mathbf{8 b}$ showed an unusual signal broadening, of the benzylic proton and all other 
protons in the benzylic aliphatic side chain. In contrast, the corresponding protons of the iPr-group in $\mathbf{8 c}$ and the benzylic proton of $\mathbf{8 d}$ showed well-resolved signals. As these effects were also visible in the corresponding ${ }^{13} \mathrm{C}$-spectra (see SI, Chapter 6) and some previously published literature spectra, ${ }^{[14]}$ we were interested in the origin of these observations. At this point two causes were taken into account: First, a coordination or even deprotonation of the benzylic protons by the counter-anion of the iodonium salt or traces of water in the NMR-solvent was considered. But the spectra in Figure 2 showed no significant effect on the peak shape or chemical shift of the aromatic methyl groups at $2.4 \mathrm{ppm}$ (green area) and a computational study of Hoffmann et al. suggested that a potential zwitterionic iodabenzene structure was very unlikely for our structures under the examined conditions. ${ }^{[19]}$ Therefore, conformational changes of our substrates were more likely to be the reason for the observed signal broadening and we investigated potential conformers via DFT calculations.

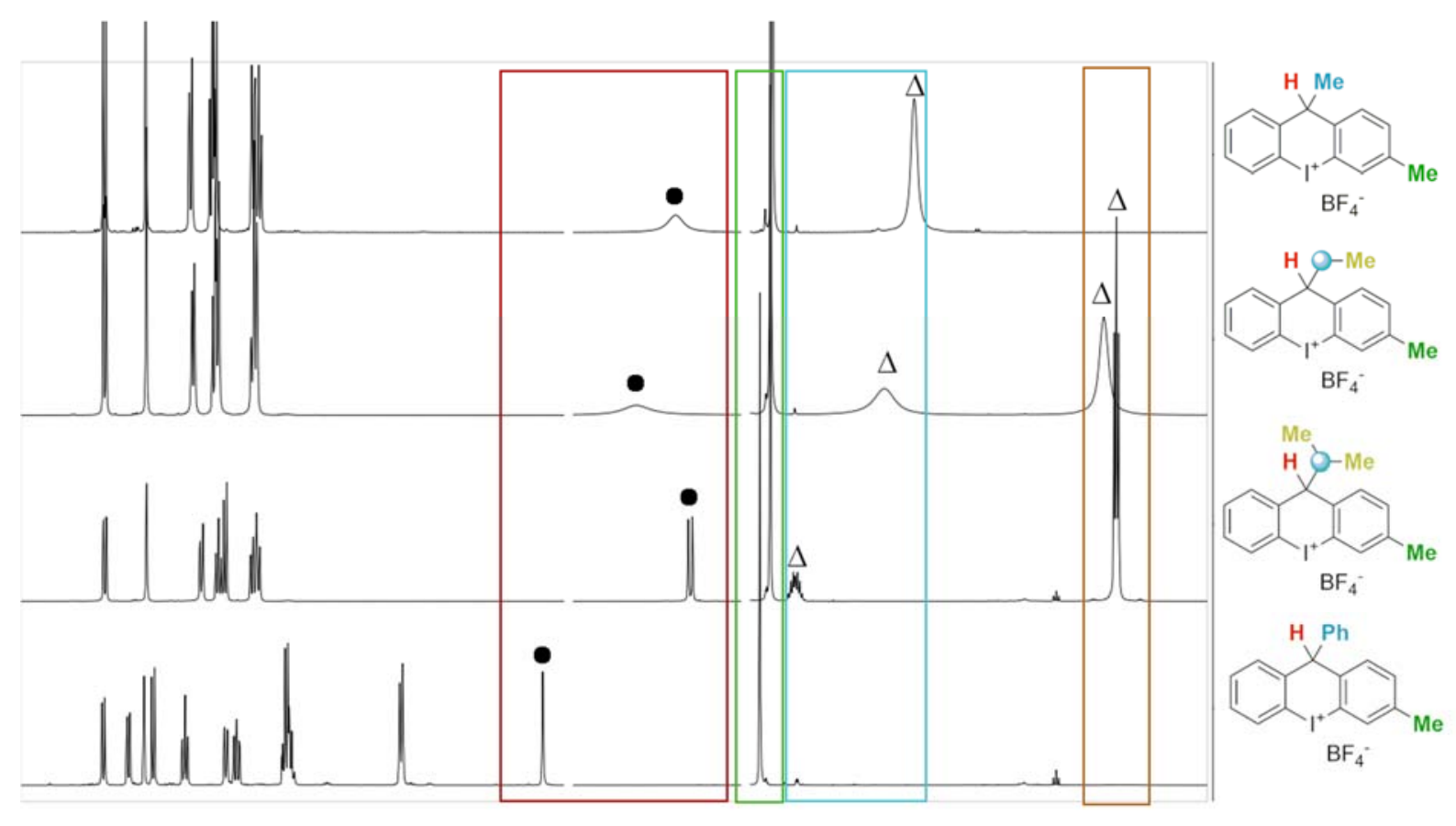

$\begin{array}{lllllllllllllllllllllllllllllll}8.4 & 8.2 & 8.0 & 7.8 & 7.6 & 7.4 & 7.2 & 7.0 & 6.8 & 6.6 & 6.4 & 6.2 & 4.6 & 4.4 & 4.2 & 4.0 & 2.4 & 2.2 & 2.0 & 1.8 & 1.6 & 1.4 & 1.2 & 1.0 & 0.8 & 0.6\end{array}$ ppm

Figure 2: $600 \mathrm{MHz}{ }^{1} \mathrm{H}-\mathrm{NMR}$-spectra of compounds 8a-d. The signals of the benzylic- $(\bullet)$ as well as alkyl-protons $(\Delta)$ are broadened in compounds $\mathbf{8 a}$ and $\mathbf{8 b}$.

As the aromatic Me-groups were unlikely to have a severe effect on conformational changes at the benzylic position, we performed our calculations on the simplified 
tetrafluoroborates 8a'-d' (Figure 3). When optimizing the structure of the Me-derivative 8a' at RI-PBE0-D3BJ/SVP level of theory, we were able to minimize two conformational isomers $\mathbf{8} \mathbf{a}^{\prime}$ ax and $\mathbf{8} \mathbf{a}^{\prime}$ eq in which the central 6-membered iodininiumring adopts a boat-like structure with the methyl group in an either axial or equatorial position. When revaluating the energies of the conformers at CPCM(DMSO)/RI-DSDBLYP/TZVPP level of theory we found that $\mathbf{8 a}$ 'ax was only $0.6 \mathrm{kcal} / \mathrm{mol}$ lower in energy than the equatorial conformer $\mathbf{8} \mathbf{a}^{\prime}$ eq. This corresponds to an equilibrium constant of $\mathrm{K} \approx 3$ that would provide a significant amount of both conformers at room temperature. While the availability of a reasonable amount of $>1 \%$ of both species is a prerequisite for the occurrence of dynamic effects in NMR-spectra (besides a significant chemical shift difference for the observed protons $\mathrm{H}_{\mathrm{ax}}$ and $\mathrm{H}_{\mathrm{eq}}$ in both conformers) we were furthermore interested in an approximate activation energy for the process.

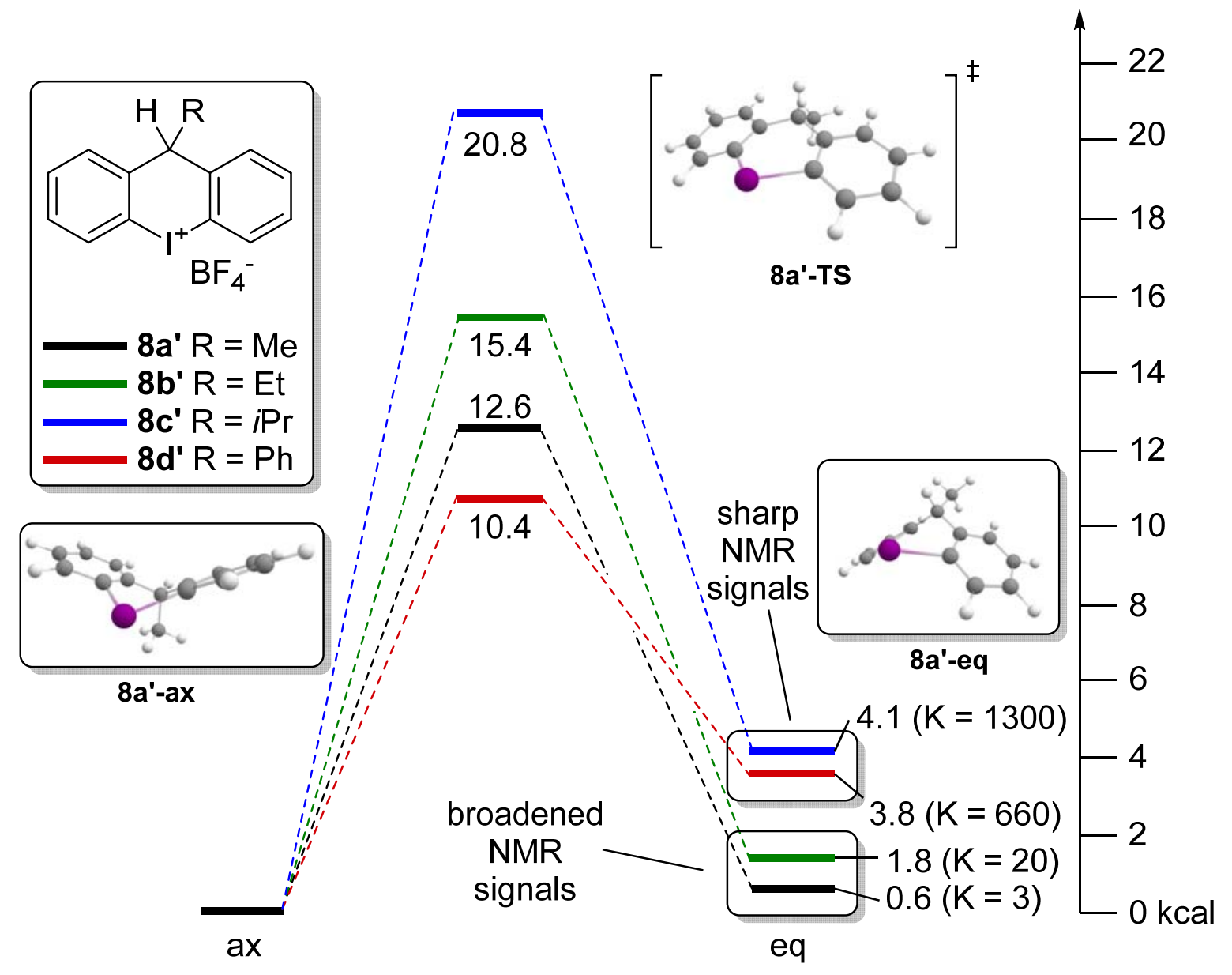

Figure 3: Relative energies in $\mathrm{kcal} / \mathrm{mol}$ for conformational isomers of dibenzo[b,e]iodininium tetrafluoroborates 8a'-d' (R = Me, Et, iPr, Ph). Energies were calculated on CPCM(DMSO)/RIDSD-BLYP/TZVPP//RI-PBE0-D3BJ/SVP level of theory. The corresponding structures for the 
methyl derivative are shown, the $\mathrm{BF}_{4}^{-}$anions were omitted for clarity. For the coordinates of all optimized structures see SI.

An optimization and energy evaluation for a transition state 8a'ts implied a free activation energy $\mathrm{G}^{\neq}$of $12.6 \mathrm{kcal} / \mathrm{mol}$ that is reasonable for the observed dynamic process at room temperature. As we could not concludingly account either the free activation energy $\mathrm{G}^{\ddagger}$ or the free energy difference $\Delta \mathrm{G}$ between the axial and equatorial conformers for the availability of sharp NMR signals, we conducted similar calculations with Et-, iPr- and Ph-substituted compounds 8b'-8d'. Since the calculated free activation energy $\mathrm{G}^{\ddagger}$ of each compound was in a range between 10.4 and $20.8 \mathrm{kcal} / \mathrm{mol}$, exchange processes should be reasonably fast. Our calculated free energy values $\Delta G$ for the conformers are in good agreement with observed signal broadenings in the spectra as the ethyl derivative $\mathbf{8 b}$ ' gives with a $\Delta \mathrm{G}$ of $\approx 1.8 \mathrm{kcal} / \mathrm{mol}$ rise to an equilibrium constant of $K \approx 20$. Calculations for $\mathbf{8} \mathbf{c}$ ' and $\mathbf{8} \mathbf{d}$ ' result in values of $K \approx 660$ and $K \approx 1300$. Thus, the most reasonable explanation for the observed signal broadening was the availability or absence of both equilibrium conformers $\mathbf{8} a \mathbf{a x}$

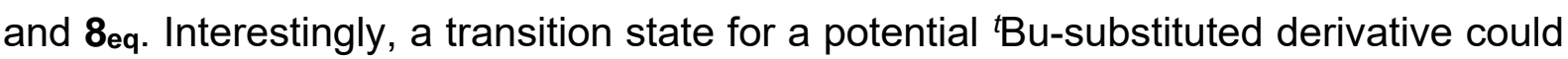
not be found which may indicate a non-interconvertibility of both isomers. This circumstance is still under ongoing investigation. 


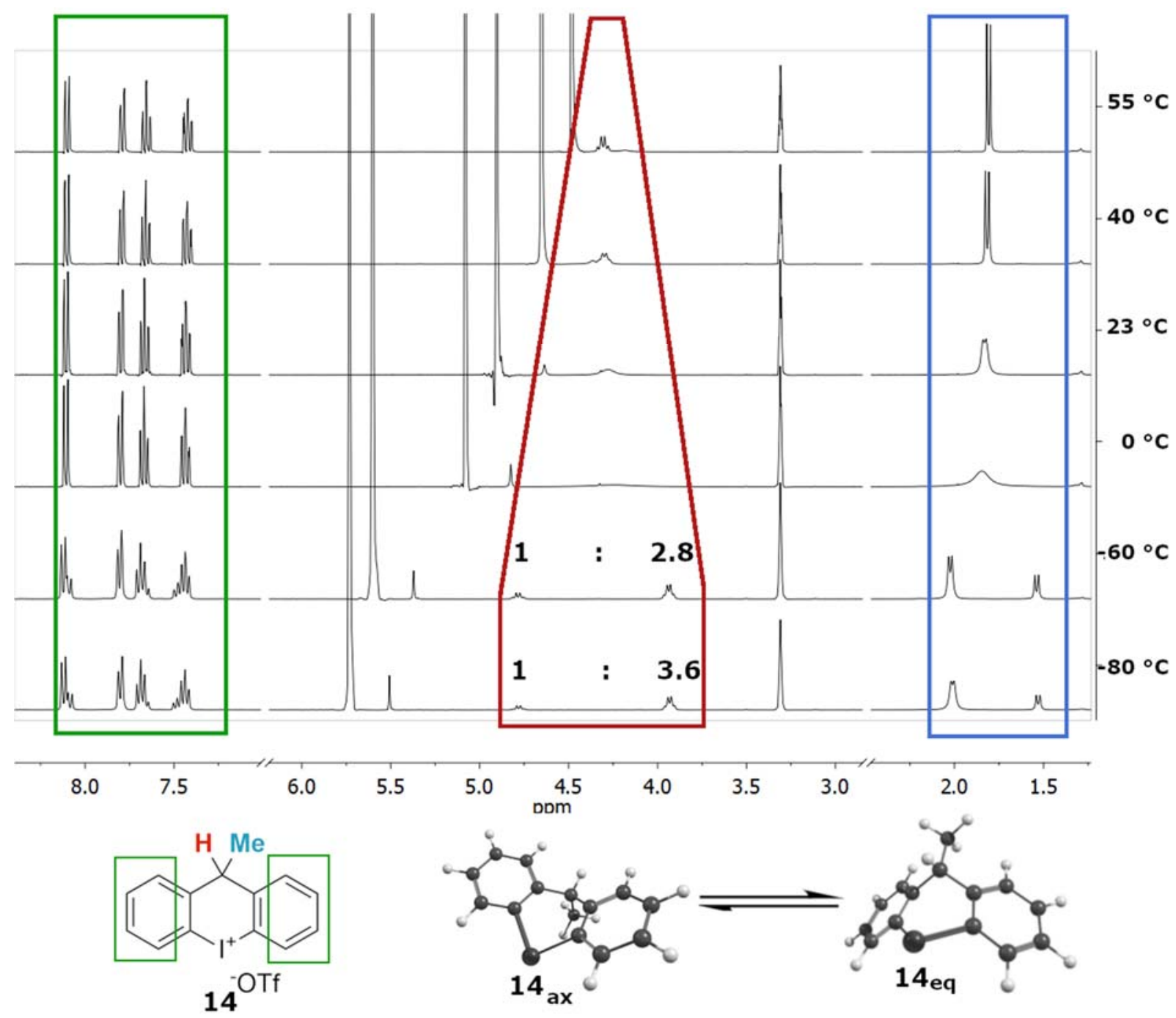

Figure 4: Top: Temperature dependant $360 \mathrm{MHz}{ }^{1} \mathrm{H}-\mathrm{NMR}$-spectra of 10-methyl-10H-dibenzo$[b, e]$ iodinin-5-ium trifluoromethanesulfonate 14 in $\mathrm{MeOH}-d_{4}$ (for synthesis see $\mathrm{SI}$ ). Bottom: Axial and equatorial conformer of the cation of 14 as optimized on RI-PBE0-D3BJ/SVP level of theory.

To back up the results of our DFT calculations we synthesized the triflate 14 as it was better soluble than the corresponding tetrafluoroborate and performed temperature dependant $360 \mathrm{MHz}-{ }^{1} \mathrm{H}-\mathrm{NMR}$ measurements in a range between -80 and $+55^{\circ} \mathrm{C}$ (Figure 4). At elevated temperatures the benzylic proton and the methyl groups gave a well separated single quartet at $4.31 \mathrm{ppm}$ and a single doublet at $1.81 \mathrm{ppm}$. Both signals showed coalescence at $\mathrm{T} \approx 0{ }^{\circ} \mathrm{C}$ and became very distinct at $-60{ }^{\circ} \mathrm{C}$ showing two quartets in a 1:2.8 ratio at 4.78 and $3.94 \mathrm{ppm}$ and two corresponding doublets at 2.02 and $1.54 \mathrm{ppm}$. The ratio changed to $1: 3.6$ at $-80^{\circ} \mathrm{C}$ what most likely originates from a different solubility of the conformers at low temperatures. 
The observed conformer ratios and the previously calculated equilibrium constant of $\mathrm{K} \approx 3$ are in good agreement. An approximate determination of the free activation energy from the measured spectra resulted in a $\Delta G^{\ddagger}$ value of $\approx 12.5 \mathrm{kcal} / \mathrm{mol}$, which is in first approximation in excellent agreement with the calculated value for 8a'. However, at this point we cannot clearly allocate the measured signals to their corresponding conformers. All attempts to assign the structures via low temperature NOE-measurements or to synthetically fix the conformation of the iodonium salt did not give resilient results and are still under current investigation.

\section{Summary}

In summary, we developed two one-pot procedures for the synthesis of 6-membered cyclic iodonium triflates and tetrafluoroborates. With a Friedel-Crafts alkylation followed by an oxidative cyclization, primary o-iodobenzyl alcohols and secondary $o$ '-iododiphenylmethanols were successfully transformed into a variety of tricyclic dibenzo[b,e]iodininium triflates in moderate to excellent yields. Delicate aliphatic 1-(2iodophenyl) alcohols could be utilized as starting materials using a modified procedure with $\mathrm{HBF}_{4}$ as the Brønsted acid. Depending on the substitution of the bridging carbon atom, we observed significant signal broadenings in the corresponding ${ }^{1} \mathrm{H}-\mathrm{NMR}$ and ${ }^{13} \mathrm{C}$-NMR spectra and verified via DFT-calculations and temperature-dependent NMRmeasurements that a fast and so far undescribed conformational switch between two stable isomers bearing the aliphatic side chain either in an axial or equatorial position are the rational for this behaviour.

\section{Experimental Section}

Detailed optimization studies, experimental procedures, analytical data $\left({ }^{1} \mathrm{H}-,{ }^{13} \mathrm{C}\right.$ - and ${ }^{19} \mathrm{~F}$-NMR-chemical shifts, IR-bands, melting points) including the corresponding NMR-spectra as well as coordinates of the calculated structures can be found in the supporting information.

\section{Acknowledgement}


Financial support by the Fonds der Chemischen Industrie (FCl) is gratefully acknowledged. 


\section{References}

[1] a) L. D. Caspers, B. J. Nachtsheim, Chem. Asian. J. 2018, 13, 1231; b) J. Charpentier, N. Früh, A. Togni, Chem. Rev. 2015, 115, 650; c) X. Li, P. Chen, G. Liu, Beilstein J. Org. Chem. 2018, 14, 1813; d) G. K. Murphy, L. Racicot, M. S. Carle, Asian J. Org. Chem. 2018, 7, 837; e) A. Yoshimura, V. V. Zhdankin, Chem. Rev. 2016, 116, 3328; f) Y. Yoshimura, H. Wakamatsu, Y. Natori, Y. Saito, N. Minakawa, Beilstein J. Org. Chem. 2018, 14, 1595.

[2] a) M. Ciufolini, N. Braun, S. Canesi, M. Ousmer, J. Chang, D. Chai, Synthesis 2007, 2007, 3759; b) S. Ghosh, S. Pradhan, I. Chatterjee, Beilstein J. Org. Chem. 2018, 14, 1244; c) H. Hamamoto, Y. Shiozaki, H. Nambu, K. Hata, H. Tohma, Y. Kita, Chem. Eur. J. 2004, 10, 4977; d) G. Jacquemot, G. Maertens, S. Canesi, Chem. Eur. J. 2015, 21, 7713; e) G. Maertens, C. L'Homme, S. Canesi, Front. Chem. 2014, 2, 115; f) L. F. Silva, B. Olofsson, Nat. Prod. Rep. 2011, 28, 1722.

[3] a) A. H. Abazid, B. J. Nachtsheim, Angew. Chem. Int. Ed. 2020, 59, 1479; b) A. Boelke, B. J. Nachtsheim, Adv. Synth. Catal. 2020, 362, 184; c) E. Cots, A. Flores, R. M. Romero, K. Muñiz, ChemSusChem 2019, 12, 3028; d) A. Flores, E. Cots, J. Bergès, K. Muñiz, Adv. Synth. Catal. 2019, 361, 2; e) C. Gelis, A. Dumoulin, M. Bekkaye, L. Neuville, G. Masson, Org. Lett. 2017, 19, 278; f) L. Massignan, X. Tan, T. H. Meyer, R. Kuniyil, A. M. Messinis, L. Ackermann, Angew. Chem. Int. Ed. 2020, 59, 3184; g) P. Mizar, A. Laverny, M. El-Sherbini, U. Farid, M. Brown, F. Malmedy, T. Wirth, Chem. Eur. J. 2014, 20, 9910.

[4] a) A. Baralle, L. Fensterbank, J.-P. Goddard, C. Ollivier, Chem. Eur. J. 2013, 19, 10809; b) N. Declas, F. Le Vaillant, J. Waser, Org. Lett. 2019, 21, 524; c) F. Le Vaillant, M. Garreau, S. Nicolai, G. Gryn'ova, C. Corminboeuf, J. Waser, Chem. Sci. 2018, 9, 5883; d) L. Li, S. Guo, Q. Wang, J. Zhu, Org. Lett. 2019, 21, 5462; e) S. Mukherjee, R. A. Garza-Sanchez, A. Tlahuext-Aca, F. Glorius, Angew. Chem. Int. Ed. 2017, 56, 14723; f) D. Wang, J. Mao, C. Zhu, Chem. Sci. 2018, 9, 5805.

[5] a) K. Aradi, B. Tóth, G. Tolnai, Z. Novák, Synlett 2016, 27, 1456; b) E. A. Merritt, B. Olofsson, Angew. Chem. Int. Ed. 2009, 48, 9052.

[6] T. Okuyama, T. Takino, T. Sueda, M. Ochiai, J. Am. Chem. Soc. 1995, 117, 3360.

[7] a) S. K. Bhunia, A. Polley, R. Natarajan, R. Jana, Chem. Eur. J. 2015, 21, 16786; b) L. Y. Chan, L. Cheong, S. Kim, Org. Lett. 2013, 15, 2186; c) Z. Chen, W.-F. 
Wang, H. Yang, X.-F. Wu, Org. Lett. 2020, 22, 1980; d) R. Ghosh, E. Lindstedt, N. Jalalian, B. Olofsson, ChemistryOpen 2014, 3, 54; e) N. Jalalian, E. E. Ishikawa, L. F. Silva, B. Olofsson, Org. Lett. 2011, 13, 1552; f) D. Kumar, M. Pilania, V. Arun, S. Pooniya, Org. Biomol. Chem. 2014, 12, 6340; g) D. Li, C. Liang, Z. Jiang, J. Zhang, W.-T. Zhuo, F.-Y. Zou, W.-P. Wang, G.-L. Gao, J. Song, J. Org. Chem. 2020, 85, 2733; h) R. J. Mayer, A. R. Ofial, H. Mayr, C. Y. Legault, J. Am. Chem. Soc. 2020; i) S. G. Modha, M. F. Greaney, J. Am. Chem. Soc. 2015, 137, 1416; j) X. Pang, Z. Lou, M. Li, L. Wen, C. Chen, Eur. J. Org. Chem. 2015, 2015, 3361; k) M. Reitti, P. Villo, B. Olofsson, Angew. Chem. Int. Ed. 2016, 55, 8928; I) D.-T. D. Tang, K. D. Collins, J. B. Ernst, F. Glorius, Angew. Chem. Int. Ed. 2014, 53, 1809; m) B. R. Vaddula, R. S. Varma, J. Leazer, Eur. J. Org. Chem. 2012, 2012, 6852; n) A. M. Wagner, M. S. Sanford, J. Org. Chem. 2014, 79, 2263; o) D. Wang, B. Ge, L. Li, J. Shan, Y. Ding, J. Org. Chem. 2014, 79, 8607; p) K. Yin, R. Zhang, Org. Lett. 2017, 19, 1530.

[8] A. Boelke, P. Finkbeiner, B. J. Nachtsheim, Beilstein J. Org. Chem. 2018, 14, 1263.

[9] a) N. Chatterjee, A. Goswami, Eur. J. Org. Chem. 2017, 2017, 3023; b) V. V. Grushin, Chem. Soc. Rev. 2000, 29, 315.

[10]a) M. Hou, R. Deng, Z. Gu, Org. Lett. 2018, 20, 5779; b) Q. Li, M. Zhang, S. Zhan, Z. Gu, Org. Lett. 2019, 21, 6374; c) S. Xu, K. Zhao, Z. Gu, Adv. Synth. Catal. 2018, 360, 3877; d) K. Zhao, L. Duan, S. Xu, J. Jiang, Y. Fu, Z. Gu, Chem 2018, 4, 599; e) K. Zhu, K. Xu, Q. Fang, Y. Wang, B. Tang, F. Zhang, ACS Catal. 2019, 9, 4951. [11]a) S. Riedmüller, B. J. Nachtsheim, Beilstein J. Org. Chem. 2013, 9, 1202; b) M. Shimizu, M. Ogawa, T. Tamagawa, R. Shigitani, M. Nakatani, Y. Nakano, Eur. J. Org. Chem. 2016, 2016, 2785; c) D. Zhu, Y. Wu, B. Wu, B. Luo, A. Ganesan, F.H. Wu, R. Pi, P. Huang, S. Wen, Org. Lett. 2014, 16, 2350.

[12]M. Wang, S. Chen, X. Jiang, Org. Lett. 2017, 19, 4916.

[13]M. Wang, Q. Fan, X. Jiang, Org. Lett. 2018, 20, 216.

[14]B. Li, Z. Chao, C. Li, Z. Gu, J. Am. Chem. Soc. 2018, 140, 9400.

[15]D. Zhu, M. Li, Z. Wu, Y. Du, B. Luo, P. Huang, S. Wen, Eur. J. Org. Chem. 2019, 2019, 4566.

[16]F. M. Beringer, L. Kravetz, G. B. Topliss, J. Org. Chem. 1965, 30, 1141.

[17]a) C. He, X. Zhang, R. Huang, J. Pan, J. Li, X. Ling, Y. Xiong, X. Zhu, Tetrahedron Lett. 2014, 55, 4458; b) J. A. McCubbin, O. V. Krokhin, Tetrahedron Lett. 2010, 51 , 
2447; c) X. Mo, J. Yakiwchuk, J. Dansereau, J. A. McCubbin, D. G. Hall, J. Am. Chem. Soc. 2015, 137, 9694; d) M. Rueping, B. J. Nachtsheim, W. leawsuwan, Adv. Synth. Catal. 2006, 348, 1033; e) M. Wilsdorf, D. Leichnitz, H.-U. Reissig, Org. Lett. 2013, 15, 2494.

[18]a) N. Soldatova, P. Postnikov, O. Kukurina, V. V. Zhdankin, A. Yoshimura, T. Wirth, M. S. Yusubov, Beilstein J. Org. Chem. 2018, 14, 849; b) N. Soldatova, P. Postnikov, O. Kukurina, V. V. Zhdankin, A. Yoshimura, T. Wirth, M. S. Yusubov, ChemistryOpen 2017, 6, 18.

[19]A. M. Rawashdeh, P. Chakkingal Parambil, T. Zeng, R. Hoffmann, J. Am. Chem. Soc. 2017, 139, 7124. 
